Göttingen Series in Biophysics

\title{
Jana Hanke
}

The Influence of Substrate Elasticity and Shear Rate on Human Blood Platelet Contraction

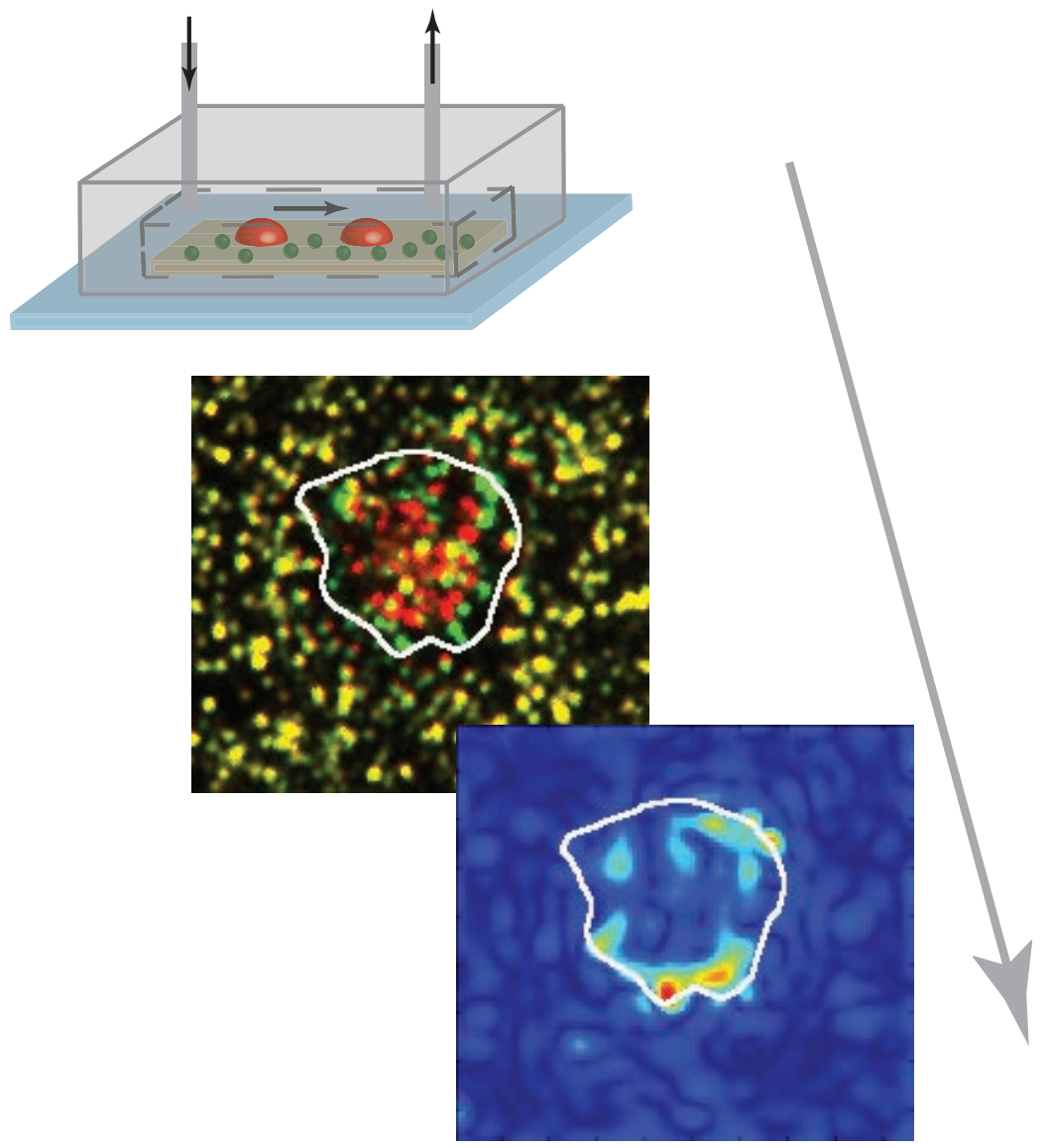

Universitätsverlag Göttingen 



$$
\text { Jana Hanke }
$$

The Influence of Substrate Elasticity and Shear Rate on

Human Blood Platelet Contraction

This work is licensed under a

Creative Commons Attribution-ShareAlike 4.0 International License.

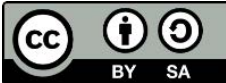


Published in 2018 by Universitätsverlag Göttingen as volume 2 in the series "Göttingen Series in Biophysics" 
Jana Hanke

The Influence of Substrate Elasticity and Shear Rate on Human Blood Platelet Contraction

Time Resolved Data Acquisition, Microfluidic Designs and

Algorithms

Göttingen Series in Biophysics

Volume 2

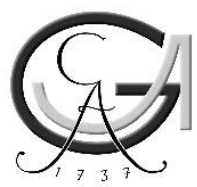

Universitätsverlag Göttingen 2018 
Bibliographische Information der Deutschen Nationalbibliothek

The Deutsche Nationalbibliothek lists this publication in the Deutsche Nationalbibliografie; detailed bibliographic data are available on the Internet at http://dnb.dnb.de.

This work was supported by the SFB 937 "Collective behavior of soft and biological matter" (project A12).

Address of the author

Jana Hanke

Email: jana.hanke@web.de

Dissertation for the award of the degree "Doctor rerum naturalium" of the Georg-August-Universität Göttingen within the doctoral program

Göttingen Graduate School for Neurosciences, Biophysics, and Molecular Biosciences (GGNB) of the Georg-August Universität School of Science (GAUSS)

Members of the thesis committee:

Prof. Dr. Sarah Köster

Prof. Dr. Claudia Steinem

Prof. Dr. Stefan Hell

This work is protected by German Intellectual Property Right Law.

It is also available as an Open Access version through the publisher's homepage and the Göttingen University Catalogue (GUK) at the Göttingen State and University Library (http://www.sub.uni-goettingen.de).

The license terms of the online version apply.

Set and layout: Jana Hanke

Cover: Jutta Pabst

Cover image: Jana Hanke

(C) 2018 Universitätsverlag Göttingen

https://univerlag.uni-goettingen.de

ISBN: 978-3-86395-389-8

DOI: https://doi.org/10.17875/gup2019-1128

e-ISSN: 2626-207X 


\section{Preface of the series editors}

The Göttingen Series in Biophysics is intended as a collection of research monographs in biological physics, carried out at the Institute for X-Ray Physics at the University of Göttingen, and in the framework of related research networks and collaborations. The series covers topics from molecular to cellular biophysics, includes experimental, numerical then theoretical work and combines methods development and studies of complex biological systems.

In most cases, the contributions are based on PhD theses. With this book series, we intend to promote the individual monographs by putting them into the larger context of the research field. We hope to be able to enhance the visibility of the research carried out here, and to encourage others to build on our findings, use our methods and advance the filed in a collaborative spirit.

Prof. Dr. Sarah Köster

Prof. Dr. Tim Salditt

Editors

Göttingen, August 2018 



\section{Contents}

1. Introduction 1

2. State of the Art 5

2.1. Blood Platelets and Their Role in Haemostasis . . . . . . . . . . . 5

2.1.1. Blood Platelets Structure and Origin . . . . . . . . . 5

2.1.2. Haemostasis . . . . . . . . . . . . . . 8

2.2. Force Measurements of Contractile Cells . . . . . . . . . . . . . . . 10

2.2.1. Experimental Set-Ups to Measure Cellular Forces . . . . . . 10

2.2.2. Different Analysis Algorithms for TFM . . . . . . . . . . . 13

2.2.3. Insights Gained from Force Measurements . . . . . . . . . . 16

2.3. Microfluidics in Biology and Cellular Forces Measured Under Shear Flow .......................... 18

3. Theory $\mathbf{2 5}$

3.1. Elasticity Theory . . . . . . . . . . . . . . . 25

3.2. Force Dipole. . . . . . . . . . . . . . . . . . . . 29

3.3. Fluid Dynamics . . . . . . . . . . . . . . . . . 31

3.3.1. Fluid Flow in Microfluidic Channels . . . . . . . . . . . 33

3.3.2. Mass Transport in Microfluidic Channels . . . . . . . . . 34

3.4. Stresses in a Cell . . . . . . . . . . . . . . . . . . . . . . 35

3.5. Modelling a Contractile Cell . . . . . . . . . . . . . . 37

4. Materials and Methods $\quad 41$

4.1. Platelet Isolation From Plasma Concentrates . . . . . . . . . . . . 41

4.2. Fabrication of PAA Gels . . . . . . . . . . . . . . . 45

4.2.1. Substrates for Static Experiments . . . . . . . . . . 45

4.2.2. Substrates for Flow Experiments . . . . . . . . . . . . . 49

4.3. Assembly of Microfluidic Channels and Chambers . . . . . . . . . . 50

4.3.1. Assembly of Mixing Channels . . . . . . . . . . . . . . 51

4.3.2. Assembly of Measuring Chambers . . . . . . . . . . 53

4.4. Imaging of Contractile Platelets . . . . . . . . . . . . . . . 55

4.4.1. Fluorescence Microscopy . . . . . . . . . . . . . . . 55 
4.4.2. $\quad$ tTFM With Various Substrate Stiffnesses . . . . . . . . . . 58

4.4.3. tTFM in Combination With Microfluidics . . . . . . . . 60

5. Development of an Analysis Algorithm $\quad 67$

5.1. Construction of Artificial Auxiliary Data Set . . . . . . . . . . . 68

5.2. PIV on Image Data of Small, Highly Contractile Cells . . . . . . . 70

5.2.1. Time-Resolved PIV . . . . . . . . . . . . . 70

5.2.2. Recalculating the Displacement Fields - Tracking of Lagrangian Markers . . . . . . . . . . . . . . . . 73

5.2.3. Reduction of Errors - Double Comparisons and Symplectic Methods . . . . . . . . . . . . . . 75

5.3. Optical Flow ..................... . . 78

5.4. Regularisation in FTTC . . . . . . . . . . . . . . . . 80

5.4.1. Fourier Transforms in the Force Calculation . . . . . . . . . 80

5.4.2. On the Need of Regularisation in FTTC . . . . . . . . . . . 82

5.4.2.1. Some Facts on Ill-Posed Problems . . . . . . . . . 82

5.4.2.2. FTTC and Ill-Posedness . . . . . . . . . . . 83

5.4.3. Reduction of Image Noise . . . . . . . . . . . . . . . . 89

5.4.4. Total Force and Error Estimation . . . . . . . . . . . . . . . 91

5.4.5. FTTC Using the Optical Flow Algorithm . . . . . . . . . . 94

5.5. Force Dipole . . . . . . . . . . . . . . . . . . . 94

5.5.1. Force Dipole Calculation . . . . . . . . . . . . . . . . . 94

5.5.2. Dipole Quotient Averaging and Error Estimation . . . . . . 95

5.5.2.1. Weighted Average by Error Estimation . . . . . . 95

5.5.2.2. Average Determined via the Variance . . . . . . . 97

6. Data Analysis $\quad 99$

6.1. Calculation of Force Data from Bead Movies . . . . . . . . . . . . 99

6.1.1. Analysis Using the PIV Algorithm . . . . . . . . . . . . . . 99

6.1.2. Analysis Using the Optical Flow Approach . . . . . . . . . 103

6.2. Post-processing Analysis . . . . . . . . . . . . . . . . 105

6.2.1. Determination of Final Spread Area . . . . . . . . . . . . 105

6.2.2. Determination of Primary Oscillating Frequency . . . . . . 106

6.2.3. Determination of Force Dipole Moment Orientation and Magnitude . . . . . . . . . . . . . . . . . . . 109

6.2.4. Angle of Flow Direction vs Angle of Major Dipole Axis . . 112 
7. Results

7.1. Comparison PIV and Optical Flow Algorithms . . . . . . . . . . . 115

7.2. Contractile Behaviour on Substrata of Different Stiffness . . . . . . 118

7.2.1. Traction Force Distribution in Relation to the Cell's Spread Area . . . . . . . . . . . . . . . . . 118

7.2.2. Temporal Evolution of the Contraction . . . . . . . . . . 119

7.2.3. Dependency of the Force on the Substrate Stiffness . . . . . 122

7.2.4. Dependency of the Maximum Force on the Platelet Size . . 123

7.2.5. Force Dipole Distribution . . . . . . . . . . . . . . . 128

7.3. Blood Platelets Under Flow Conditions . . . . . . . . . . . . . . . 132

7.3.1. Device Characterisation by Simulation . . . . . . . . . 132

7.3.1.1. Simulation of the Velocities and Shear Rates Inside the Measuring Chamber . . . . . . . . . . 133

7.3.1.2. Simulation of the Diffusion and Transport Processes Inside the Mixing Channel . . . . . . . . . . 135

7.3.2. Testing for Usability of the Flow Chamber . . . . . . . . . . 137

7.3.3. Attachment of Platelets During Flow . . . . . . . . . . . . . 146

7.3.4. Contractile Behaviour Under Shear Flow . . . . . . . . . . 147

7.3.5. Adaptation of Force Orientation to the Shear Stress . . . . 153

8. Discussion

8.1. Analysis Algorithm . . . . . . . . . . . . . . . . . . . 161

8.2. Design of Microfluidic Device . . . . . . . . . . . . . . . . . 164

8.3. General Observation on Platelet Contraction . . . . . . . . . . 168

8.4. Oscillatory Contractions . . . . . . . . . . . . . . . . 171

8.5. Force Orientation Within Flow Field . . . . . . . . . . . . . . . . . 175

9. Summary and Conclusion $\quad 179$

A. Appendix: Mathematical Proofs 185

A.1. Regularisation Using PIV and Lagrangian Markers . . . . . . . . . 185

A.2. An Error Estimation for the Dipole Quotient Averaging . . . . . . 187

B. Appendix: Matlab Programmes 189

B.1. Main Programme . . . . . . . . . . . . . . . . . . . . . . . 189

B.2. Image Contrast Enhancer . . . . . . . . . . . . . . . . . 195

B.3. Drift Correction . . . . . . . . . . . . . . . . . . . 196

B.4. Calculate Velocity Fields . . . . . . . . . . . . . . . . . 197

B.5. Calculate Displacements and Traction Forces . . . . . . . . . . 199 
Contents

Bibliography 


\section{Abbreviations}

$\begin{array}{ll}\text { AFM } & \text { Atomic force microscopy } \\ \text { APS } & \text { Ammonium persulfate } \\ \text { APTMS } & \text { 3-aminopropyltrimethoxysilane } \\ \text { BSA } & \text { Bovine serum albumin } \\ \text { BF } & \text { Bright field } \\ \text { CCD } & \text { Charge-coupled device } \\ \text { Cy5 } & \text { Cyanine 5 dye } \\ \text { FITC } & \text { Fluorescein isothiocyanate } \\ \text { FOV } & \text { Field of view } \\ \text { FTTC } & \text { Fourier transform traction cytometry } \\ \text { HT buffer } & \text { Hepes-Tyrode buffer } \\ \text { KLT } & \text { Kanade-Lucas-Tomasi algorithm } \\ \text { MilliQ } & \text { distilled, ultrapure water } \\ \text { NA } & \text { Numerical aperture } \\ \text { PAA } & \text { Polyacrylamide } \\ \text { PAR } & \text { Protease activated receptors } \\ \text { PBS } & \text { Phosphate buffered saline } \\ \text { PDMS } & \text { Poly(dimethylsiloxane) } \\ \text { PGE } & \text { Prostaglandin E } \\ \text { PIV } & \text { Particle image velocimetry } \\ \text { PSG } & \text { Pipes saline glucose } \\ \text { PTV } & \text { Particle tracking velocimetry } \\ \text { RG } 1 \text { GV } & \text { Strong robust generalised cross validation } \\ \text { ROI } & \text { Region of interest } \\ \text { rpm } & \text { Rotations per minute } \\ \text { TEMED } & \text { Tetramethylethylenediamine } \\ \text { TFM } & \text { Traction force microscopy } \\ \text { VWF } & \text { Von Willebrand factor }\end{array}$





\section{Introduction}

Human blood platelets are small, anucleate cell fragments that are important for the life-saving process of haemostasis, i.e. the process of wound healing. By adhering to the extracellular matrix at the side of the wound, spreading and contracting on it, the wound is closed and a continued bleeding prevented. A disturbance of any of these steps leads to serious health impairments, in the most severe cases even to death. To understand the mechanisms governing the blood clotting in healthy humans is thus crucial to also comprehend the implications of the disturbed process. At the same time, given their reduced complexity in biological set-up, blood platelets can be used as simplified cell models to study the signalling pathways between cell and environment. Lacking both DNA and transcription factors, platelets react and adapt to the surrounding only by means of direct chemical signalling across its membrane and within the cytoplasm. The reaction of the platelets to its physical environment is the focus of this work.

The formation of a blood clot in the body takes place while the blood flows continuously within the blood vessel. The resulting shear rate experienced by the blood platelets varies greatly between large veins (starting at about $10 \mathrm{~s}^{-1}$ ) to small arteries (up to about $2000 \mathrm{~s}^{-1}$ ) under physiological conditions [99]. Simultaneously, the various tissues found in the human body that a platelet encounters all exhibit different elasticities ranging from the soft brain tissue at about $1 \mathrm{kPa}$ to the harder stiffness of about $100 \mathrm{kPa}$ of collagenous bone [28]. This directly gives rise to the question whether the difference in micro-environment, such as shear rate or elasticity, changes the reaction of the platelet in terms of adhesion or contraction. Here, we investigate the contractile behaviour of platelets and its variation if the stiffness or shear rate is changed. The forces are measured in a two-dimensional setting for single platelets using traction force microscopy. The influence of the two physical quantities is studied in two different set-ups, namely, under static as well as dynamic conditions. For the static experiment, the stiffness of the substrate is changed without additional external influences in terms of e.g. shear stress. The dynamic experiment is conducted within a microfluidic device with changing shear flows, keeping the substrate stiffness constant. Summarised, there are four major goals in this works that are addressed: 
1. The development and optimisation of an analysis algorithm for the recorded traction force data on the base of existing algorithms. Here, the small size as well as the very dynamic and strong forces of the platelets require a revision of the commonly used algorithms.

2. The measurement of the traction forces exerted by single platelets. Here, both the spatial as well as the temporal development are studied.

3. The development of a microfluidic device that allows us to measure the forces of the platelets under flow. The device is designed to not only facilitate the force measurement but to do so under conditions comparable to the previous, static experiments in terms of e.g. concentration of the trigger substance.

4. The measurement of the contractile forces of platelets under flow conditions. As with the previous experiments, we again study both the spatial and temporal evolution of the force.

Chapter 2 gives a brief summary of the architecture and function of blood platelets. Furthermore, the main experimental and analytical methods to measure traction forces exerted by cells are presented along with major insights gained from these measurements for both platelets as well as other cells. Next, the concept of microfluidics in connection to cell biology is presented. Here, a major point is to provide the already proven usability of microfluidics when studying platelets. Lastly, the first results in combining traction force microscopy and microfluidics are reported.

Subsequently, Chapter 3 summaries several physical concepts that are important for this work. This includes the basics of elasticity theory to understand the physical and mathematical aspects of traction force microscopy. We will continue with the underlying mathematics of fluid dynamics as well as mass transport in fluids. In connection to platelets being exposed to shear flow, the stresses on the cell due to the flow are described. Additionally, the theoretical concept of an elastic dipole is presented. Finally, a mathematical model to simulate the contraction of a cell or cell layer on an elastic substrate is described.

In Chapter 4, the experimental procedures are summarised. This includes the fabrication of the elastic substrates used for both experiments, the assembly of the microfluidic device as well as the imaging of the contracting platelets.

Chapter 5 presents the first, theoretical part of our results, namely the analysis algorithm. It describes the analysis step-by-step, demonstrating results of the individual steps on a specially constructed data set. The optimisation of the algorithm is reported as well as an alternative, also adapted algorithm which is later 
calibrated to our newly designed algorithm. Additionally, we introduce the force dipole ratio to determine the degree of isotropy in contraction and its evolution over time. For the practical application of the algorithms presented above, in Chapter 6 , the necessary parameters for the evaluation of the experiments are given. Additionally, several analytical approaches used to calculate, e.g., the oscillatory force frequency or final spread area are described.

This is followed in Chapter 7 by the presentation of the results for both experimental set-ups. The spatial and temporal development of the force is described and the previously introduced force dipole quotient studied. For the static experiments, the model previously introduced in Chapter 3 is used to derive an approximation for the internal stress of the platelet as well as evaluate the influence of the platelet's thickness on the mechano-sensitivity. For the microfluidic experiments, the designed measuring chamber is characterised both through simulation and measurement. Lastly, the preferred contractile direction compared to the flow direction is presented. The observations made here are discussed in the subsequent chapter, Chapter 8.

In the last Chapter 9, the major results of this work are summarised. We finish this thesis with the possible implications of our results for the process of blood clotting. 



\section{State of the Art}

In the present chapter, an overview of the current knowledge about blood platelets and their contraction is given (Section 2.1). Subsequently, different experimental and analytical methods to determine cellular forces are presented in Section 2.2. Finally, the newest advances of measuring forces under flow conditions as well as the influence of shear flow on cells in general are described in Section 2.3.

\subsection{Blood Platelets and Their Role in Haemostasis}

\subsubsection{Blood Platelets Structure and Origin}

Human blood platelets are small, anucleate cell fragments found in the blood with a life span of only 7-10 days ( [77], Chapter 3). They are one of the key players in the process of haemostasis as described previously. In the non-active state, they exhibit a discoid shape with a diameter of only $2 \mu \mathrm{m}$ to $5 \mu \mathrm{m}$ and a height of $0.5 \mu \mathrm{m}$ ( [77], Chapter 3). They are thus considerably smaller as the more commonly known cells in the blood, namely red blood cells (diameter: $7.5 \mu \mathrm{m}$ to $8.7 \mu \mathrm{m}$, height: $1.7 \mu \mathrm{m}$ to $2.2 \mu \mathrm{m}$ [23]). Under shear flow, platelets are pressed towards the blood vessel walls, allowing them to be close by in case of an injury.

The exact way of the formation of blood platelets is still a matter of research as several pathways have been proposed. Common for all of them is the fact that platelets stem from larger, polynuclear cells called megakaryocytes found in the bone marrow. It has been suggested that platelets form by a budding-of process or blebbing taking place at the outer membrane from the megakaryocyte ( $[77]$, Chapter 2).

Another model is based on the discovery of so called proplatelets, thin cytoplasmic processes developing from the megakaryocytes. These proplatelets display small, bead-like ends, connected via the cytoplasm. In the final stages of the proplatelet production, they are released from the megakaryocytes. The ends of the pro- 


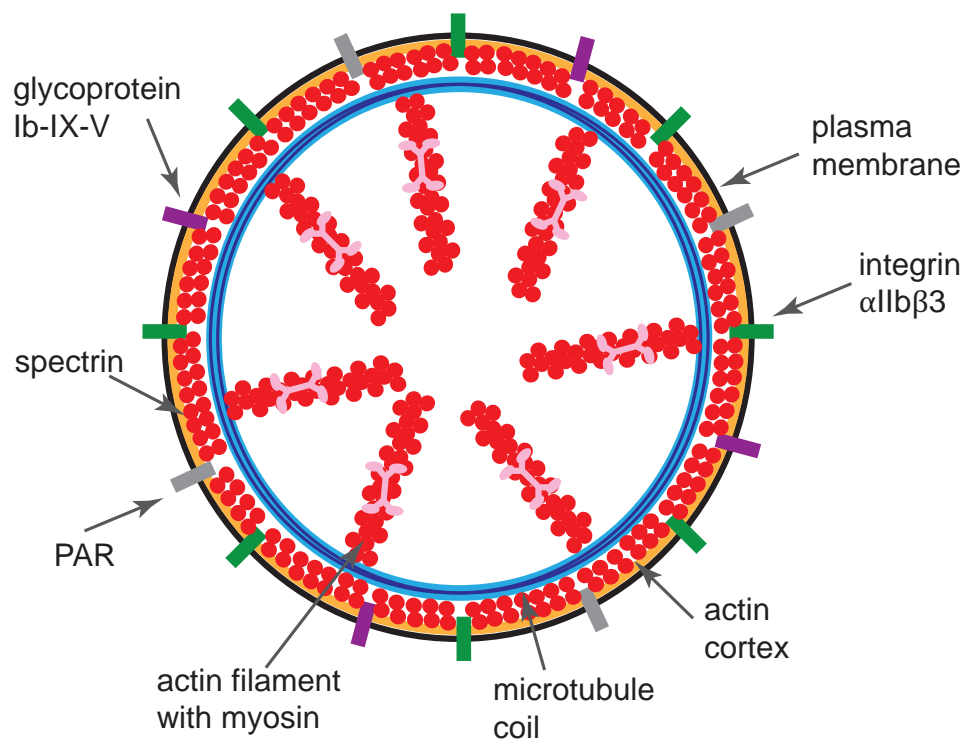

Figure 2.1.: The inner structure of a platelet. The outer shell is comprised out of a membrane (black) with a multitude of receptors and proteins, such as the integrin $\alpha I I b \beta 3$ (green), the glycoprotein GP Ib-IX-V (violet) and the thrombin receptor family PAR (grey). The open canalicular system is not shown. Underneath the membrane, first a spectrin cytoskeleton (orange) is found followed by the actin cortex (red). Further inside, a microtubule coil (blue) helps maintain the characteristic, discoid shape of the platelet. On the inside, actin filaments (red) span the cytoplasm. Associated to the filaments are the myosin motors (pink) which bind to the actin upon activation of the platelet. Image adapted from Ref. [100].

platelets now contain small microtubule coils similar to those found in platelets. It has thus been proposed that platelets form from the ends of these intermediate cells [49].

Intrinsically, platelets exist in their resting state. An sketch of the simplified structure of a platelet can be found in Fig. 2.1. In resting state, a platelet exhibits a generally smooth surface with smaller membrane indentions similar to those found in the brain. Within these indentions of the internalised plasma membrane, small openings of a tunnel-like system, the so-called open canalicular system, can be found. During spreading, the surface area of the platelet increases to span, on average, $30 \mu \mathrm{m}^{2}$ [102] and it is believed that the invaginations provide the additional membrane needed for this process. Further, the open canalicular system has been shown to transports substances from the inside of the platelets to the blood plasma and vice versa. 
While a multitude of receptors are found on the outer membrane, for this thesis, to understand the adhesion and activation, we can reduce the number to five receptors. Two of those are the glycoproteins GP Ib-IX-V and integrin $\alpha \mathrm{IIb} \beta 3$ which are responsible for the adhesion to the extracellular matrix during haemostasis. The former primarily binds to the ligand von Willebrand factor (VWF) while the latter also binds to fibrinogen. Both glycoproteins are mobile on the surface. In particular, it has been shown that in the early state of activation, the integrins are moved towards the centre of the lower membrane of the platelet ( [77], Chapter $3)$.

Additionally, platelets contain a multitude of receptors for activation. One class of receptors are the so called protease activated receptors (PAR). It has been shown that PAR1, PAR3 and PAR4 are all receptors for thrombin, an activation agonist for platelets. The binding of thrombin molecules to the PAR proteins is one way of initiating the actin polymerisation as well as the activation of the myosin motors ( [77], Chapters 9 and 16).

Underneath the plasma membrane, a spectrin-based skeleton is bound to the actin cortex. In its resting state, only about $50 \%$ of the entire actin is polymerised into filaments that stabilise the cell. Inside the actin cortex, the microtubules are found laying in several loops to form a coil, maintaining the discoidal shape [111]. Further inside, actin filaments span the interior of the platelet to act as a matrix for other organisational units. During activation and spreading, the cytoskeleton changes its configuration to undergo the observed structural changes. It has been shown that during spreading, the loops of the microtubule ring are elongated by the dynein motors. Afterwards, they start to coil, resulting in a spherical shape of the platelet $[22,111]$. At the same time, the actin is polymerised and activated myosin motors bind to the filaments to facilitate the contraction of the platelet.

Lastly, a number of organelles are found within the platelets. Most interesting here are the $\alpha$ granules and dense granules. Both structures are storage units within the cell, where the former are larger in size. The amount of $\alpha$ granules is dependent on the size of the platelet and each contains a number of proteins such as von Willebrand factor and fibrinogen. Dense granules are storage units for smaller molecules, especially for calcium, ATP and ADP. 


\subsubsection{Haemostasis}

Under normal conditions, that is without damage to the blood vessel walls, platelets are found in their resting or quiescent state, exhibiting a discoid shape. To avoid an activation at this stage, the blood contains molecules that actively hinder the triggering, such as e.g. prostacyclin. Interestingly, for the work presented here, the receptor on the platelet membrane that binds to prostacyclin also reacts to prostaglandin E1 ( $\mathrm{PGE}_{1}$, [77], Chapter 13).

Upon injury to the vessel walls, a chain of reactions is started to form a blood clot which is initiated by the activation of platelets. The clot formation can, simplified, be divided into three major parts: the initialisation, the extension and the stabilisation. The three stages are shown in Fig. 2.2. On a cellular level, an injury means a detachment of endothelial cell at the wall which exposes the extracellular matrix. This matrix consists of collagen as well as its ligand von Willebrand factor. As the platelets are pressed to the side of the vessel wall due to the shear flow, they are immobilised on the matrix surface and activated by the collagen-VWR complex or free-flowing thrombin (Fig. 2.2 A). The activation describes two different pathways. While the collagen-VWR complex reacts with the aforementioned glycoproteins GP Ib-IX-V and integrin $\alpha \mathrm{Ilb} \beta 3$, thrombin reacts with the PAR receptors. Both reactions, however, result in an increase of intracellular calcium concentration which activates integrin $\alpha \operatorname{IIb} \beta 3$ and thus the platelet ( [77], Chapter 16).

The activation of the platelet starts a cascade of structural changes. Its shape changes from a discoid shape to a spherical shape. The intracellular calcium triggers not only the integrin but also the polymerisation of actin filaments. The platelet spreads on the extracellular matrix via the formation of both filopodia and lamellipodia [101] and the extension of the folded membrane. Their final spread shape resembles that of a fried-egg shape. Examples of spread platelets may be found in Fig. 2.3. Simultaneously, myosin motors IIa and IIb associated with the actin filaments are also activated by the intracellular calcium, assisted by the activation by thrombin and its following reaction cascade. Thus, during spreading, a contractile, actomyosin driven force is observed (Ref. [77], Chapter $4,[29])$. This process is known as clot retraction. At the end of the initialisation step, a monolayer of activated platelets is found on the site of injury.

On the monolayer of blood platelets, the blood clot is now extended by recruitment of other platelets from the blood flow (Fig. 2.2 B). The recruitment is chemically controlled by the release of agonists from the platelets as well as local generation 
A

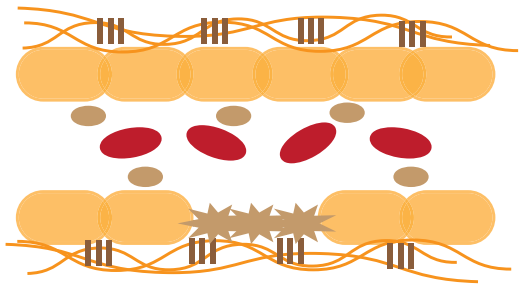

C

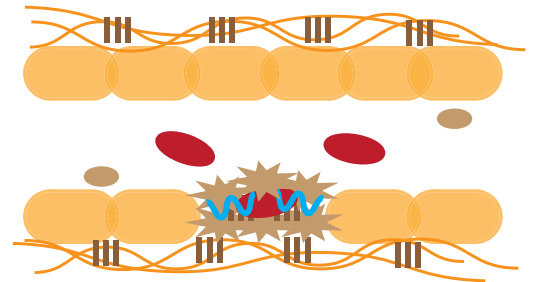

B

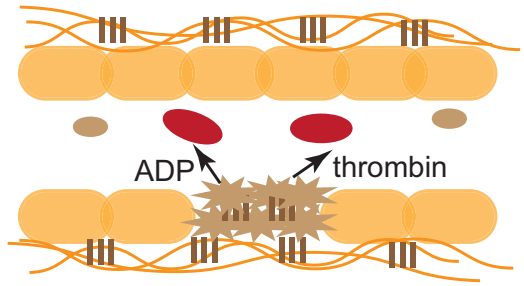

Figure 2.2.: The process of wound healing. A During the initialisation of the blood clot, the platelets start to bind to the extracellular matrix composed out of collagen and VWF to form a monolayer of cells. Following, the arrested platelets release signal substances such as e.g. ADP to recruit other platelets to the blood clot $(\boldsymbol{B})$. Free thrombin molecules assist in this process. Finally, the blood clot stabilises, containing also fibrinogen and captured red blood cells (C). Image adapted from Ref. [77], Chapter 16.
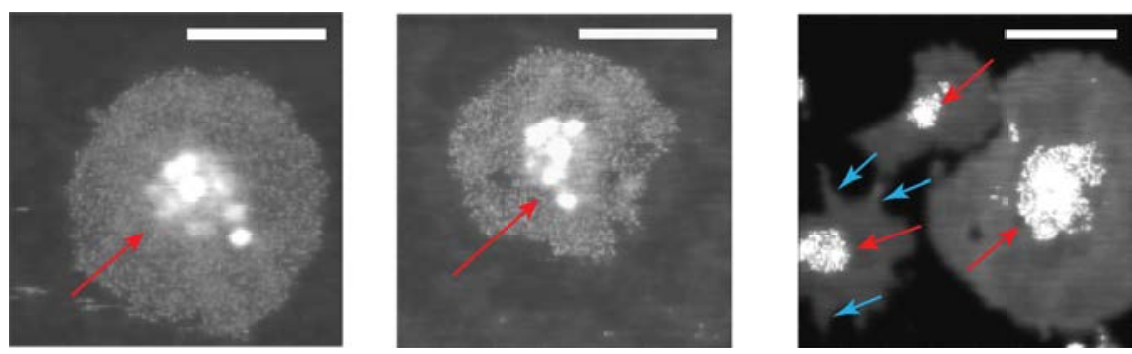

Figure 2.3.: Examples of spread platelets seeded on glass and recorded using a confocal microscope. The membrane was stained before seeding with the dye dSQ12S (Ref. [14]), at a concentration of about $300 \mathrm{nM}$. Most platelets here show the typical round structure associated with fully spread platelets. The high intensity in the middle of the platelets (red arrow) denotes the increased height in the membrane due to assembly of the organelles. The last image was taken on a gold coated surface and shows an ensemble of cells. Here, also filopodia (blue arrows) can be seen. The smaller light streaks outside the cells are bypassing platelets. Scale bar: $5 \mu \mathrm{m}$. Images were recorded in cooperation with A. Chizhik, Third Institute of Physics, University of Göttingen. 
of thrombin. The agonists are first stored within the granules described above and released upon calcium increase. The connection between platelets is facilitated by fibrinogen to which they can bind via activated integrin $\alpha \operatorname{Ib} \beta 3$. After the high levels of activation signalling are decreased, tight bonds between the platelets within the plug are built inside a meshwork of fibrinogen. This stabilises the clot and prevents further bleeding. Additionally, captured red blood cells are found within the final blood clot (Fig. 2.2 C).

In this thesis, we are interested in the stage at which the platelet spreads on the matrix. In particular, it is known that during this process, the cells do not only spread but also contract ( [77], Chapter 4). It has previously been shown that the stiffness of the environment affects the spreading of platelets in that they increase their spread area with stiffness [93]. This effect stagnates at a stiffness of about $50 \mathrm{kPa}$. Additionally, the number of platelets attached per area increases in a similar way as the spread area. It has thus been suggested that the contractile force may vary in a similar fashion [94]. Another interesting point is the influence of continuous flow during haemostasis. A number of studies have been conducted which demonstrated that the shear rate during blood clot formation greatly influences the process (e.g. Refs. [16,34,60,83,103]). Hence, the contraction of platelets may also be influenced by the shear rate.

To measure the forces exerted by a cell, a multitude of experimental and analytical tools have been developed. In the following section, a short overview of these different approaches is given. Additionally, results gained from force measurements are presented. Here, observations both for platelets as well as other cells are summarised.

\subsection{Force Measurements of Contractile Cells}

\subsubsection{Experimental Set-Ups to Measure Cellular Forces}

Cellular forces have been shown to be of importance in various biological processes such as cell migration [69], proliferation and differentiation [28]. To measure the cellular forces during these processes, different methods have been developed, both from an experimental as well as from an analytical point of view.

One of the earliest methods to visualise forces exerted by adherent cells was the imaging of soft elastic substrates. If cells located on the substrate contracted, the gel was deformed, resulting in a wrinkly surface [41]. To be able to quantify the forces corresponding to the degree of 'wrinkling', more accurate imaging techniques 
A

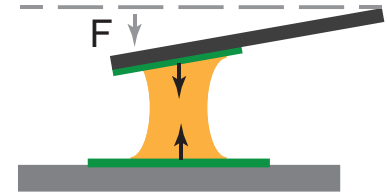

C

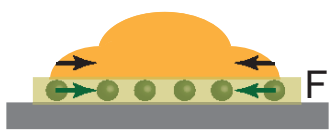

B

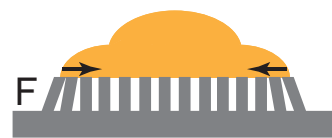

D

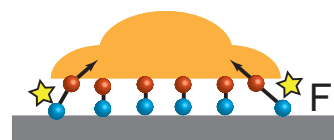

Figure 2.4.: Four different methods to study the force $F$ generated by a cell (orange). The direction of force measurement is given by the black arrows. A AFM measurements are conducted by determining the deflection in a cantilever upon cell contraction. To facilitate this kind of measurement, adhesive proteins (green) are often used on the surface and cantilever. $\boldsymbol{B}$ Cells can be grown on micro-post arrays and the deflection of the single post reflects the contraction. Contrary to AFM, this is done in two dimensions. $C$ Also measured in two dimensions is the deformations of elastic substrates underneath a cell (TFM). The beads within the substrate (green) are displaced accordingly to the exerted force above them (green arrows). $\boldsymbol{D}$ A localised force measurement technique involves the use of molecular force sensors (blue-red spheres). Upon pulling on the molecule, it is stretched, thus increasing the distance between the active parts (both spheres). Following, either the change in energy transfer is measured (two fluorophores) or the direct change in fluorescent intensity (one fluorophore and one quencher).

had to be found. Generally, four different methods for force measuring experiments have developed over time: atomic force microscopy (AFM) measurements (e.g. [58, $78]$ ), micro-post arrays (e.g. [29, 57, 65, 116, 120]), continuous elastic substrates containing markers (e.g. $[17,20,43,74,88,107,122])$ and, most recently, fluorescent, spring-like force sensor molecules (e.g. $[31,125,127])$. A sketch of all methods may be found in Fig. 2.4.

To measure the force exerted along an axis, atomic force microscopy or similar set-ups can be used (e.g. [58,78], Fig. 2.4 A). The cell is grown between a substrate and a flexible microplate [78] or cantilever [58]. By changing the stiffness of the flexible part of the set-up, the response of the cell to the stiffness can be determined. Also, detachment forces from the cell to the substrate may be measured by actively applying an external force. However, as already mentioned, this approach measures the forces along a one-dimensional axis. 
A two-dimensional tool to measure the contractile forces is the so called micropost array (e.g. $[29,57,65,116,120]$, Fig. 2.4 B). A micro-post array consists of a number of elastic, deformable posts positioned close to each other. The single posts are usually cast from Poly(dimethylsiloxane) (PDMS) and vary in post size, density and stiffness [120]. They are regularly distributed to facilitate easy tracking of deformation. Further, the posts are coated with adhesion proteins such as fibrin to facilitate attachment. By micro-contact printing the adhesive proteins to the posts in defined geometries, the cell's shape and cytoskeletal organisation can be actively controlled [57]. Contracting cells seeded on the array deflect the underlying micro-posts, enabling the observer to directly re-calculate the exerted force. These assays are either conducted on ensembles of cells [29,65] or on a single-cell basis $[57,116,120]$. Note that this approach yields a measurement at discrete locations, namely the positions of the micro-posts.

Instead of using the discrete, two-dimensional set-up of micropost arrays, continuous substrates can be used, a technique called traction force microscopy (TFM, e.g. $[17,20,43,74,88,107,122]$, Fig. 2.4 C). These substrates are cast from PDMS [17, $43]$ or, most commonly, polyacrylamide (PAA, [81,88,107,122]). Within the substrate, fluorescent markers are embedded to visualise the deformation of the substrate during cell contraction. Contrary to the micropost array, each fluorescent marker within the continuous gel yields a contribution to the measurement of the entire deformation field. Keeping in mind that the size of the fluorescent beads in general varies between a few tens to hundreds of nanometres in diameter, this gives a much higher resolution of the force field. The resolution in force measurement is limited by the density of beads, the imaging resolution as well as the employed analysis algorithm. At the same time, as all previously described force measurement tools, the stiffness of the substrate can be varied, here in terms of the degree of cross-linking in the polymer gels. The fabrication of PAA gels have been adapted to also include micro-contact printed proteins as for the micro-post arrays $[74,81,88,122]$ as well as force measurement in three dimensions $[21,62]$. Using this experimental set-up, keep in mind that due to the continuity of the substrate, deformations are not only observed at the point of force transduction on the gel but also in its direct neighbourhood.

To measure the forces at the actual point of force transmission, in recent years, so called tension sensor molecules have been developed (e.g. [31, 125, 127], Fig. 2.4 D). Simplified, these sensors consist of two fluorescent molecules connected by a spring-like molecule. When the two fluorophores are close together (= the "spring" is relaxed), an active energy exchange occurs between them and can be measured 
by using Förster resonance energy transfer (FRET). The further the molecules are apart, less energy transfer is seen. Depending on the folding properties of the connecting molecule, different force ranges can be covered with a single sensor, often spanning an interval of some piconewtons [31], contrary to the nanonewtons measured in the methods described above. Alternatively to two fluorophores, one can be exchanged by a gold particle to quench the flourescence of the other [105, 127]. Developing these sensors is still an ongoing research topic as it has to be guaranteed that advert effects such as hysteresis do not occur during multiple stretching cycles.

In the presented work, the most common force measurement technique, TFM in combination with continuous PAA substrates, is used. This allows us to study the force development of a single platelet both in a two-dimensional space as well as in time. PAA substrates have the advantage of being well tunable in stiffness between $100 \mathrm{~Pa}$ and $100 \mathrm{kPa}$ [115] which corresponds to the elasticity range found in the human body [28]. It has previously been demonstrated that platelets adhere well to these substrates when coated with fibrinogen $[93,107]$.

In TFM, one interesting point apart from the experimental set-up is the way to analyse the given data. Independent of the dimensionality of the set-up, the initial point of analysis is a given set of images of beads changing their position. To re-calculate the forces exerted by the cell on the gel from the bead positions, a multitude of algorithms have been developed and are presented in the following.

\subsubsection{Different Analysis Algorithms for TFM}

All algorithms to calculate the exerted forces of the cell when using continuous substrates are based on elasticity theory [59]. In technical terms, one determines the displacement of the markers in the gel and from there re-calculates the corresponding forces. Note that the deformation of the gel is always taken towards a so called null-image, an image taken of the un-deformed substrate. Most commonly, the null-image is recorded by trypsination $[50,88]$ or other forms of cell relaxation drugs [80] after the recording. In cases of migrating cells, the un-deformed substrate is recorded when the cells have left the recording frame [20]. When the adherence of cells is also imaged, the point of attachment acts as a reference frame [107]. For micro-contact printed substrates, the un-deformed fluorescent protein patterns is taken as a null-image $[81,122]$. 
To determine the displacement within the substrate, different analysis approaches have been developed: particle tracking velocimetry (PTV; e.g. $[61,62,98,107]$ ), particle image velocimetry (PIV; e.g. $[3,20,21,46,69,88,95,114,117])$ and, more recently, an optical flow method (e.g. $[45,71,75])$.

PTV involves direct bead tracking within the image and is, mathematically seen, a Lagrangian approach to solve the problem of the displacement field calculation. Each bead is identified in the null-image and the deformed image and the most likely match between them is found. This may be done by triangulation $[62,107]$ or correlation-based [98]. PTV has been adapted to include both time-resolved measurements [107] as well as three-dimensional recordings [61,62]. The computational complexity here depends heavily on the number of beads as well as the number of images that are compared. Furthermore, with increasing bead density, the reliable identification of the beads between images becomes more and more difficult, especially when using triangulation. One way to circumvent this problem is the introduction of beads of multiple colours [98] but that is not possible for all applications. Hence, this approach, while intuitive and easily applicable for sparse bead densities, fails for problems of tightly packed tracers.

Most commonly used is the PIV algorithm [69,88,95,114]. Here, the single images are sub-divided into smaller square windows and the entire included bead pattern is studied. By calculating the cross-correlation between windows at equal position, the statistically most probable translation is determined. This approach assumes that within the sub-window, approximately, all pixels move linearly in the same direction. Note that the window grid is static for all images for this algorithm to work and is thus an Eulerian method. This approach allows for a higher bead density of a single colour to be used as no bead identification is needed. Indeed, only intensity patterns in space are utilised at this point. Hence, a higher spatial resolution may be reached here. Additionally, PIV has been adapted to include both time-resolution $[3,20,46,117]$ and three-dimensional spatial resolution [21]. The limiting factor from a computational point of view is here the choice of subwindows. The size is dependent on the degree of deformation of the substrate which in turn depend on the substrate stiffness, the magnitude of the exerted force as well as of the time difference between images that are compared. Consequently, for large enough deformations, a cross-correlation between sub-windows becomes impossible to determine. Further, with an increasing number of sub-windows, the computational cost increases as well. 
A more recent algorithm in connection with TFM is the optical flow method [45, 71,75]. Similar to the PTV, single beads or features are found in the images that are to be compared. Around these features, similar to the PIV algorithm, small sub-windows are defined which are tracked. Note that here no regular, static grid is used but a set of irregularly spaced, translating windows. Yet, it is still assumed that neighbouring pixels move similar to each other and the deformations are small enough to be able to to track them reliably. Most importantly however, this algorithm requires that the brightness of the feature that is tracked does not change significantly between images. It has been shown to work reliably and with good resolution on two-dimensional substrates [45, 75].

Having calculated the displacement field by either method, the traction forces are now determined. The underlying equation is, however, the inverse of the Boussinesq solution to the equation of equilibrium of an elastic medium bounded by a plane ( [59], §1.8 and [105]). The inverse is generally not readily calculated in real space but in Fourier space. Hence, traction forces are often calculated in Fourier space by using Fourier Transform Traction Cytometry (FTTC; [10]). In most cases, the FTTC algorithm involves the solution of an ill-posed problem. Thus, regularisation schemes need to be included in these cases $[98,105]$. Regularisation can in some cases be avoided or reduced if a-priori knowledge of the traction force field exists. To this end, adapted algorithms such as e.g. the model-based TFM [110] or traction reconstruction with point forces [114] have been developed. Here, knowledge of the cellular, internal structure or, in the latter case, the distribution of focal adhesions is used to better localise the actual point of force exertion.

In the presented work, the PIV algorithm is used. Given the vast amount of studies that have already used it, the flexibility of the algorithm has been demonstrated. Here, however, the algorithm is enhanced to be applicable to the small size of the platelets, their very dynamic and strong forces as well as the temporal resolution. To validate the results gained from this adapted PIV approach, it is compared to a similarly adapted optical flow method. For the force reconstruction, this is followed by the FTTC algorithm.

Some primary cells, in most cases considerably larger than platelets, have been studied as to their adaptation to the micro-environment. In particular, the reaction of the cells to substrates or micro-post arrays of different stiffnesses in terms of force development was investigated. In the following, major insights gained from these studies are presented. 


\subsubsection{Insights Gained from Force Measurements}

A considerable number of studies observed the behaviour of different primary cells when presented with various surroundings. In particular, the contractile force in dependency of the environment's stiffness was of interest. For cells such as fibroblasts [69], endothelial cells [12], Jurkat T-cells [46], neutrophils [89] and also cancer cells [55], it was shown that the forces they exert on the underlying substrate increase with increasing stiffness. This adaptation demonstrates an active feed-back loop within the cells that first sense the stiffness and then regulates a corresponding response by, e.g., changing the internal cellular structure.

Other examples of an active adaptation process within the cells was observed in the speed of their contractile response. In Trichet et al. [120], it could be shown that the velocity of contraction increases with the stiffness. Simultaneously, the cells' spread area also increased with the rigidity. Furthermore, the greater the cell's area was, the higher the exerted force was measured to be $[12,35]$.

The different observation methods were adapted to also include smaller cells such as dictyostelium cells $[20,21]$ and T-cells [46]. Compared to larger cells such as e.g. fibroblasts or endothelial cells, it was shown that they exert considerably less force. Additionally, for dictyostelium cells, it was demonstrated that they not only exert forces in a planar direction but also perpendicular to the substrate surface.

On a molecular level, it was revealed by a combination of knock-down and inhibition experiments in combination with force measurements that both actin polymerisation as well as myosin-driven contraction contribute to the exerted forces of a cell $[11,46]$. Which of the two mechanisms contribute more to the contraction can, however, vary between different cell lines. As an example, while mouse embryonic fibroblast cell could be shown to be driven to $90 \%$ by myosin activity [11], the traction forces observed from T-cells decreased significantly when the actin polymerisation was inhibited [46].

For platelets, most of the different experimental methods were employed to measure the forces exerted by the activated, contracting cells. In particular, continuous PAA substrates [81, 107], AFM measurements [58], micro-post arrays [29, 65] and, most recently, tension sensors [125] have been applied. Note that, due to their size, experiments conducted on micro-post arrays used small aggregates of platelets while the other experiments were conducted on single platelets. All of the different approaches aimed to answer the question which force a platelet can exert on the surrounding during contraction. The resulting forces varied considerably depending on the approach chosen. 
In Schwarz Henriques et al. [107], a substrate of stiffness $4 \mathrm{kPa}$ was utilised and the development of the force studied over time. Here, the point of attachment was taken as the point of reference for the un-stressed substrate. A comparatively sparse bead density was used to measure the contractile forces. It was shown that the platelets, on average, yielded a force of about $34 \mathrm{nN}$. Further, the force developed towards a force plateau that was reached within 25 min. The contraction was described as directed towards the cell centre and close to isotropic behaviour. It was also shown that the total force scaled with the total spread area.

In contrast to the continuous substrate used above, Lam et al. [58] used an AFM to measure the axial contraction. The stiffness of the set-up was calculated to be $12 \mathrm{kPa}, 29 \mathrm{kPa}$ and infinitely stiff for different experiments. For the single platelets, the maximum contraction force was determined to be between $1.5 \mathrm{nN}$ and $79 \mathrm{nN}$, reached within $10 \mathrm{~min}$ to $15 \mathrm{~min}$. Interestingly, the averaged maximal forces per stiffness were lower than the forces measured on the PAA substrates above, in particular about $18 \mathrm{nN}$. It was further observed that the platelets adapted to the rigidity of the substrate by exerting more force with increasing stiffness. Additionally, the adhesion force was measured to be about $70 \mathrm{nN}$. The platelet's elasticity was calculated to be $10 \mathrm{kPa}$, with an increasing stiffness for cells that exerted a higher force. In a following work to this experiment, Myers et al. [81] exchanged the AFM to fibrinogen patterned PAA substrates. Contrary to the substrates used by Schwarz Henriques et al. [107], the platelets were only allowed to adhere between two fibrinogen patches instead of an evenly coated substrate. The force was calculated by the changes in position of the protein patches. Although the PAA gel provided a two-dimensional base for the experiment, due to the protein patterns, the force was determined in a uni-axial manner, similar to the AFM approach. The stiffness was tuned between $25 \mathrm{kPa}$ and $100 \mathrm{kPa}$ and the thrombin concentration varied from $0.1 \mathrm{u} / \mathrm{mL}$ to $5 \mathrm{u} / \mathrm{mL}$. They found that the magnitude of forces not only depend on the stiffness of the substrate but also on the thrombin concentration with an optimum found for the stiffness at $75 \mathrm{kPa}$ or a thrombin concentration of $5 \mathrm{u} / \mathrm{mL}$. The average force ranged from the $15 \mathrm{nN}$ to about $40 \mathrm{nN}$ depending on the stiffness and thrombin combination.

Next, in Refs. [29,65], micro-post arrays were used. Liang et al. [65] determined the stiffness of the micro-posts to be about 2.9 MPa. Here, different thrombin concentrations were utilised to study the dependency of the contraction process on the amount of stimulus. It was shown that an increase in thrombin concentration yields a higher force response until a concentration of $3.5 \mathrm{u} / \mathrm{ml}$ after which no change was seen. At the same time, the clot volume also stagnates in size. Over 
time, with a constant supply of thrombin and platelets, the clot increased in size and contractile force. The force per platelet in the contracting clot was estimated to be about $2 \mathrm{nN}$, considerably less than the works conducted on PAA substrates or with an AFM. Feghhi et al. [29] used posts of a stiffness of about 2.5 MPa and determined the force exerted by the clot on each micro-post. They measured a force of about $14 \mathrm{nN}$ per post and three to four posts per clot. Instead of determining the total force per platelet, they wanted to test the influence of myosin IIa on the contraction process. By blocking different activation pathways for myosin, it was shown that this motor is essential for force generation and clot retraction.

Most recently, Wang et al. [125] developed a molecular tension sensor for the force transmitted by the $\alpha \operatorname{IIb} \beta 3$ integrin. This sensor was demonstrated to tunable between $10 \mathrm{pN}$ and $60 \mathrm{pN}$. Using this sensor, they revealed that the force magnitude of platelets determines the force distribution. Integrins of low tension, i.e. below $54 \mathrm{pN}$, were distributed in a ring-like pattern around the periphery with two to three spots of higher force. Integrins exhibiting higher tensions were only found in two to three focus points. The latter were co-localised with vinculin, a protein associated with focal adhesions. Lastly, they demonstrated that the lower forces develop directly at the beginning of the adhesion and spreading process while the higher, focused forces first appear at later time-points.

\subsection{Microfluidics in Biology and Cellular Forces Measured Under Shear Flow}

During recent years, cellular responses in terms of e.g. differentiation (e.g. [13]) or migration (e.g. $[32,50])$ to actively controlled external conditions have been increasingly investigated. Apart from the static experiments described in the previous section, often, this involves microfluidic techniques. In microfluidics, small quantities of fluid are flushed over the experimental set-up in a controlled fashion. Additionally to only needing small quantities of chemical and biological material, the advantage of a microfluidic approach is the small Reynolds number associated with this method. Consequently, only laminar flow regimes are observed, facilitating an easier control of e.g. the shear rate or concentration of trigger substances. Another advantage is the down scaling of several physical quantities that define the experiment, such as the diffusion time, which allows for faster response times [124]. The microfluidic devices are often produced from PDMS, varying greatly in the geometry depending on the specific influence factor one is interested 
in to study [124]. As an example, one factor frequently studied is the influence of different molecules including drugs on cells (e.g. $[13,32,50,82])$. Using simple set-ups of straight channels with one inlet and one outlet, the addition of specific drugs can be temporally and spatially controlled and the changes in morphology or activity in cells observed. With more complex channel structures of, e.g., multiple mixing regions $[13,50,52]$, defined chemical gradients can be produced $[32,82]$. This allows the user to observe the cellular response such as chemotaxis [50] in dependency of the drug's concentration.

Another influence factor that may be studied is, naturally, the shear stress and shear rate itself during flow (e.g. $[19,57,79])$. Here, both the orientation in flow for adherent cells $[57,79,92]$ as well as the direction of movement in motile cells [19] have been studied. These studies have in common that a preferential direction in parallel to the flow was observed. The alignment of the movement to flow for, e.g. dictyostelium discoideum, was shown to be dependent on the shear stress the cells were exposed to where the directionality increases with increasing stress [19]. Endothelial cells also align towards the flow [57] while the cytoskeleton including the focal adhesion sites shifted with the flow.

For platelets, microfluidics has been used to study to major steps in haemostasis, namely the adhesion to the extracellular matrix (e.g. $[34,38,39,82,84])$ as well as the formation of the thrombus (e.g.. [16, 18,60,83]). Apart from studying the direct influence of shear flow on the formation of a thrombus, the proteins involved have been studied. In the following, the major insights thus gained are presented. In the context of adhesion under flow conditions, both ways to facilitate adhesion (e.g. $[34,38,39,84])$ as well as activation pathways $[82,84]$ were investigated. Savage et al. [103] showed that at low shear rates of $50 \mathrm{~s}^{-1}$, the arrest of platelets is governed by the reaction to fibrinogen. At higher shear rates of $1500 \mathrm{~s}^{-1}$, however, platelets start to adhere to VWF but not fibrinogen. Gutierrez et al. [34] reached a similar result using mouse platelets. They demonstrated that the reaction of integrin $\alpha \operatorname{Ib} \beta 3$ to the extracellular matrix is sufficient at venous shear stress (about $1 \mathrm{~Pa}$ ) but not at higher shear stress. Additionally, it was shown that integrin $\alpha \operatorname{IIb} \beta 3$ is necessary for thrombus formation on collagen. Hansen et al. [39] studied the attachment of platelets on collagen spots within the physiological range (shear rate $50 \mathrm{~s}^{-1}$ to $920 \mathrm{~s}^{-1}$ ), an alternative adhesion protein. They revealed that a minimum diameter of $20 \mu \mathrm{m}$ of collagen is necessary for platelets to arrest on it. Furthermore, they demonstrated that adhesion and aggregation increases with the shear rate until about $300 \mathrm{~s}^{-1}$, after which it stagnates. Previously, 
they had already shown that an increase in collagen concentration on the surface dramatically increases the attachment rate of platelets under arterial shear rate $\left(1000 \mathrm{~s}^{-1}\right)[38]$.

Adding to the attachment observations at high shear rates $\left(1800 \mathrm{~s}^{-1}\right)$ and adaptation pathways, Nesbitt et al. [84] studied the calcium signalling occurring between integrin $\alpha \operatorname{IIb} \beta 3$ and glycoprotein Ib-V-IX during adhesion to VWF. They found that platelets can be divided into three groups in terms of intracellular calcium content where an increased calcium level corresponds to an increase in attachment rate and oscillatory calcium flux. It was suggested that a minimum level of intracellular calcium is necessary to establish stable adhesions under shear flow. The reaction between glycoprotein Ib-V-IX and VWF was proposed as an initiator to integrin $\alpha \operatorname{IIb} \beta 3$ activation by raising the calcium levels within the platelet.

The activation of platelets within the blood flow is highly dependent on the presence of different agonists such as thrombin or ADP ( [77], Chapter 16). A microfluidic tool to study this influence in a pressure controlled manner was developed by Neeves and Diamond [82]. Keeping the shear rate constant $\left(250 \mathrm{~s}^{-1}\right)$, they increased the ADP level within the flow chamber to study the ADP-dependent adhesion and aggregation. They observed that with increasing ADP content, the platelets went from non-adhesive to first building monolayers and finally aggregates.

To summarise briefly, the adhesion process of platelets has been shown to be governed by several external factors. Depending on the shear rate, different matrix proteins facilitate the attachment. At low, venous shear rates, the adhesion depends on the reaction of integrin $\alpha \operatorname{IIb} \beta 3$ to fibrinogen. At higher shear rates, immobilised fibrinogen does not result in attachment but VWF does. Alternatively, collagen works for both low and higher shear rates. At the same time, the concentration and, where relevant, patch size of proteins and trigger substances is important. Generally, a higher concentration of either matrix protein or trigger substance yields a higher response of the platelets although saturation occurs. Simultaneously, a minimal patch size of matrix protein is necessary to facilitate a reaction. However, platelets differ between each other in their response to external signals even under equal conditions, as observed with the calcium levels of single platelets.

Following the initial adhesion, platelets start to aggregate and form a thrombus. This process was investigated by various groups using microfluidic techniques (e.g. $[16,18,60,83])$. Lee et al. [60] demonstrated that the formation of a thrombus is directly influenced by the shear rate. The activation of platelets by shear stress 
was achieved by stirring and the platelets showed an irreversible activation and aggregation at high shear rates. The aggregate size increases with increasing shear rate until about $3000 \mathrm{~s}^{-1}$ after which it decreases again. Also studying the influence of shear rates (between $100 \mathrm{~s}^{-1}$ and $1000 \mathrm{~s}^{-1}$ ) on the thrombus formation and its stability, Neeves et al. [83] let murine platelets aggregate on patterned collagen. Like the previous work by Lee et al. [60], they revealed that the thrombus size first increases until middle high shear rates before decreasing again. Note that Neeves' experiment used lower shear rates than Lee did even though their results were similar. Stepping up the flow to a shear rate of $8000 \mathrm{~s}^{-1}$, the thrombus stability was tested. All aggregates showed a decrease in size. However, actively stimulating the PAR4 receptor reduced the loss in size at high shear rate, suggesting that thrombin increases the stability under flow.

Combining the influence of shear rate and adhesion proteins, Conant et al. [16] developed a microfluidic tool which allowed them to observe the aggregation of platelets in parallel channels at different shear stresses and channel coatings. At lower shear stresses (1-2 Pa) within the channel, they demonstrated an aggregation on collagen I but not VWF or BSA (Bovine serum albumin). At pathologically high shear stress (up to $20 \mathrm{~Pa}$ ), however, thrombus formation on VWF was observed. Excluding the influence of the shear stress on the aggregation, Van de Walle et al. [18] studied the adhesion to and especially aggregation on fibrinogen under physiological conditions (shear rate of $100 \mathrm{~s}^{-1}$ ) more in detail. In particular, they were interested in how the spatial distribution of fibrinogen influences the adhesion and aggregate formation. They did show that fibrinogen lines parallel to the flow with spacings of $6 \mu \mathrm{m}$ allows for platelet aggregates to build between them as bridges. Increasing the distance between the fibrinogen lines gradually to $30 \mu \mathrm{m}$ reduced the presence of aggregates. Simultaneously, it was demonstrated that fibrinogen patches of a width of $4 \mu \mathrm{m}$ or less in flow direction decreases the attachment and aggregation.

To recap, it was demonstrated that the aggregation of platelets is heavily dependent on the shear rate. With increasing shear rate, the clot size increases until a maximum level is reached. Increasing the shear rate beyond this limit produces the opposite effect by reducing the size again. The latter effect can, however, be partly reversed by simultaneously stimulating the PAR4 receptor, an additional activation receptor. This suggests that also the activation by trigger substances play a role in the ability to form aggregates and withstand external shear stresses. As already discussed previously in the context of adhesion, aggregation is also governed by matrix proteins. Again, different proteins facilitate the formation 
of clots depending on the shear rate. Similarly to the adhesion to collagen, the aggregation on fibrinogen is also controlled by the patch size where a minimum adhesion area is necessary to facilitate the clot formation.

Using microfluidic techniques, many insights into the adhesion and aggregation process of platelets have been gained. While the molecular pathways have been studied in great detail, no information exists on how the contraction of a thrombus or a single platelet depends on the shear flow. While Myers et al. [81] included their PAA substrate into a microfluidic channel, they did not include any flow until after the platelets were attached and spread. They reported that the force did not change from the static experiments, suggesting that the critical time point for the force development is the time point of attachment. To understand the process of wound healing, the study of the entire process under flow is thus of essence.

Within the last 10 years, microfluidic set-ups have been combined with TFM to measure the forces exerted by different cells. Here, the reaction of fibroblasts [17], neutrophils [50] and endothelial cells $[47,57,92]$ to shear stress was studied in detail. In the latter case, this is especially interesting in the context of angiogenesis. These experiments were conducted by incorporating substrates as used in the static TFM case into the microfluidic channel. In the case of Das et al. [17], the substrate was a continuous PDMS substrate containing fluorescent markers while Lam et al. [57] used micropost arrays made out of PDMS. Both approaches facilitated the bounding of the chambers by oxidising the PDMS with an oxygen plasma. Perrault et al. [92] and Hur et al. [47] incorporated continuous PAA gels into chambers held together externally. Jannat et al. [50] also used PAA gels but bound the glass slide with the substrate to the PDMS cast by plasma activation. However, protein coating of the substrate was done after binding by flushing the closed microfluidic systems.

For the TFM experiments conducted with endothelial cells, shear rates as found within veins were applied $[91,99]$. Not only the traction forces were measured but also the alignment compared to the flow direction. As mentioned above, the cell body of attached cells was found to align to a parallel orientation [57] compared to the flow, contrary to the perpedicular direction which was reported for fibroblasts in three-dimensional tissue cultures $[85,86]$. The traction forces measured exhibited a tendency to a perpendicular orientation, although large variations could be observed. Note that for all experiments, the endothelial cells were first left under static conditions to adhere to the substrates. While the combination of microfluidics and TFM has been used in the recent past as described above, none of them has tried to study the entire process of cellular attachment and force development. 
Neither did the conducted measurements include a high temporal resolution of small cells, a crucial requirement when observing the attachment and contraction process of platelets. 



\section{Theory}

In this chapter, an overview of the physical background to the methods used in the presented work is given. This includes a brief overview of the principal concepts of elasticity theory (Section 3.1) including the Boussinesq solution applied for traction force microscopy. Next, we describe the physical quantity of a force dipole and its interpretation in terms of isotropic contraction (Section 3.2). As we also want to study our cells under flow conditions as mentioned previously, the basics of fluid dynamics and mass transport in fluids are established in Section 3.3. From the previously described physical concepts, we briefly discuss stresses acting on elastic objects found under flow conditions (Section 3.4). Further, a model is introduced that characterises the contraction of a cell or cell layer on an elastic substrate (Section 3.5).

\subsection{Elasticity Theory}

One of the major physical methods that is used in this work is the calculation of the traction forces exerted by a cell on an elastic substrate by measuring the displacement of fluorescent beads. To understand how one can translate displacements to forces, in the following, a brief description of the basic equations of elasticity theory is given. To start, assume we have a deformable body, e.g. an infinitesimal volume (see Fig. $3.1 \mathrm{~A}$ ), on which an external force is applied. From here, let us introduce the concepts of strain and stress. For the following derivations, we always assume small displacements or deformations. This assumption allows us to linearise our equations.

Upon application of a force on the body, it will start to deform, i.e. elements within the body change their position. Let us regard two points at positions $\mathbf{x}$ and $\mathbf{x}+d \mathbf{x}$. During deformation, they will change their position by $\mathbf{u}(\mathbf{x})$ and $\mathbf{u}(\mathbf{x}+d \mathbf{x})$. The squared distance between them in the deformed body is then 
A

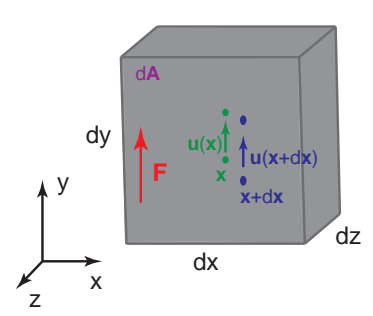

B

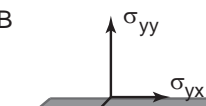

C

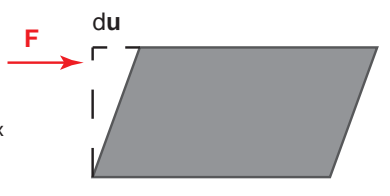

Figure 3.1.: A An infinitesimal deformable volume to which a force (red) is applied. Two example points are included which show the change in position due to the resulting deformation. The original positions are denoted as $\mathbf{x}$ and $\mathbf{x}+d \mathbf{x}$ and their displacements $\mathbf{u}(\mathbf{x})$ and $\mathbf{u}(\mathbf{x}+d \mathbf{x})$, respectively. The resulting stresses are shown in $\boldsymbol{B}$ on the three visible sides. Lastly, in $\boldsymbol{C}$, a sheared body is shown.

given by

$$
\begin{aligned}
d^{2} & =\sum_{i}\left(d x_{i}+u_{i}(\mathbf{x}+d \mathbf{x})-u_{i}(\mathbf{x})\right)^{2} \\
& =\{\text { Taylor expansion }\} \\
& =\sum_{i} d x_{i}^{2}+2 \sum_{i, j} d x_{i} \frac{\partial u_{i}}{\partial x_{j}} d x_{j}+\sum_{i, j, k} \frac{\partial u_{i}}{\partial x_{j}} d x_{j} \frac{\partial u_{i}}{\partial x_{k}} d x_{k}
\end{aligned}
$$

where the subscripts denote the $i, j, k$ th component of the corresponding vector. Note that the first term is equal to the original distance between the points in the un-deformed body. Hence, the change within the body is only given by the latter two terms. We can then define the single components of the strain tensor $\epsilon$ as

$$
\epsilon_{i j}=\frac{1}{2}\left(\frac{\partial u_{i}}{\partial x_{j}}+\frac{\partial u_{j}}{\partial x_{i}}+\sum_{k} \frac{\partial u_{k}}{\partial x_{i}} \frac{\partial u_{k}}{\partial x_{j}}\right)
$$

Thus, the strain describes the degree of deformation within a body. We can further see that the tensor is symmetric such that $\epsilon_{i j}=\epsilon_{j i}$. Lastly, as we assume small displacements and hence small strains, this means that we can neglect the last 
term in Eq. (3.4) and reduce the strain to

$$
\epsilon_{i j}=\frac{1}{2}\left(\frac{\partial u_{i}}{\partial x_{j}}+\frac{\partial u_{j}}{\partial x_{i}}\right) .
$$

Having now defined the strain of our body, let us consider the term stress. For an un-deformed body, it is said that the body is in mechanical equilibrium, i.e., the resultant force on any point in the body is zero. When the body is deformed, it leaves the equilibrium. This gives rise to internal forces that act towards the equilibrium state, the so called stresses. We can thus define the stress tensor $\boldsymbol{\sigma}$ (compare Fig. 3.1 B) by

$$
\sigma_{i j}=\frac{F_{i}}{\partial x_{j}}
$$

It was shown by Parodi and Pershan that there always exists a transformation such that it holds that $\sigma_{i j}=\sigma_{j i}([59] \S 1.2)$. For isotropic materials under stress along one axis, the relation between the stress and the strain is given by

$$
\begin{aligned}
E \cdot \epsilon & =\sigma \\
\nu E & =\epsilon_{t}
\end{aligned}
$$

where $E$ denotes Young's modulus in the unit of pressure, $\nu$ the Poisson's ratio (dimensionless) and $\epsilon_{t}$ the transversal strain. The Young's modulus as well as the Poisson's ratio are material properties. $E$ describes the elastic stiffness of a material. As an example, the Young's modulus for tissues found in the body is of the order of $\mathrm{kPa}$ while materials such as wood or metal are in the order of GPa. The Poisson's ratio $\nu$ describes the ratio between transverse strain and axial strain. In other words, it compares the compression in one direction if the body is stretched in the perpendicular direction. For most materials, $\nu$ falls in the range between 0 and $\frac{1}{2}$. A material of Poisson's ratio $\frac{1}{2}$ corresponds to an incompressible material.

The above relations between strain and stress (Eq. (3.8)) hold true for uni-axial stress. If one considers a linear elastic material under stress in all three dimensions, the single components are related such that

$$
\boldsymbol{\epsilon}=\frac{1+\nu}{E} \boldsymbol{\sigma}-\frac{\nu}{E}(\operatorname{trace}(\boldsymbol{\sigma}))
$$

which corresponds to Hook's law. Here, $\operatorname{trace}(\boldsymbol{\sigma})$ denotes the sum over all elements in $\boldsymbol{\sigma}$ along the diagonal. 
We have now introduced all physical quantities we will need to correlate displacements and forces applied to an elastic body. Let us first consider the body in equilibrium. We can express the equation in equilibrium as

$$
\Delta \mathbf{u}+\frac{1}{1-2 \sigma} \nabla(\nabla \cdot \mathbf{u})=-\rho \mathbf{g} \frac{2(1+\sigma)}{E}
$$

with $\rho$ being the density of the body, $\mathbf{g}$ the gravity and $\mathbf{u}$ the displacement as before (Ref. [59], §1.7). In our specific case, we always study a body that is deformed by an external force applied to the surface. Then, Eq. (3.10) is rewritten as

$$
(1-2 \sigma) \Delta \mathbf{u}+\nabla(\nabla \cdot \mathbf{u})=0
$$

where the force is only included in the boundary conditions (Ref. [59], §1.7).

In traction force microscopy, the problem is reduced to having a force or force distribution $f$ applied on an elastic substrate of a certain height, width and length. In particular, the height or thickness of the substrate is considerably larger than the observed displacements due to the contracting cell. So, again, we can use the assumption of small deformations. Moreover, the area occupied by the cell is much smaller than the substrate area such that there are no deformations near the substrates boundary. Thus, we can consider the substrate to occupy an infinite half-space. This approach allows us to solve the elasticity equation (Eq. (3.11)) analytically leading to an integral equation connecting surface displacements and surface forces.

Let the substrate occupy the half-space $\left\{\mathbf{x}=(x, y, z) \in \mathbb{R}^{3} \mid z>0\right\}$. So the $x$ and $y$-coordinates span the surface while $z$ starts at the substrate surface and is pointing into it. For this situation, Boussinesq (compare Ref. [59], §1.8) has derived the solution

$$
\int_{\mathbb{R}^{3}} \mathbf{G}\left(\mathbf{x}^{\prime}-\mathbf{x}\right) f\left(\mathbf{x}^{\prime}\right) d \mathbf{x}^{\prime}=\mathbf{u}(\mathbf{x}) .
$$

The Green's function $\mathbf{G}$ is given by

$\mathbf{G}(\mathbf{x})=\frac{1+\nu}{2 \pi E}\left(\begin{array}{ccc}\frac{2(1-\nu) r+z}{r(r+z)}+\frac{2 r(\nu r+z)+z^{2}}{r^{3}(r+z)^{2}} x^{2} & \frac{2 r(\nu r+z)+z^{2}}{r^{3}(r+z)^{2}} x y & \left(\frac{z}{r^{3}}-\frac{1-2 \nu}{r(r+z)}\right) x \\ \frac{2 r(\nu r+z)+z^{2}}{r^{3}(r+z)^{2}} x y & \frac{2(1-\nu) r+z}{r(r+z)}+\frac{2 r(\nu r+z)+z^{2}}{r^{3}(r+z)^{2}} y^{2} & \left(\frac{z}{r^{3}}-\frac{1-2 \nu}{r(r+z)}\right) y \\ \left(\frac{z}{r^{3}}+\frac{1-2 \nu}{r(r+z)}\right) x & \left(\frac{z}{r^{3}}+\frac{1-2 \nu}{r(r+z)}\right) y & \frac{z^{2}}{r^{3}}+\frac{2(1-\nu)}{r}\end{array}\right)$

where $r=\sqrt{x^{2}+y^{2}+z^{2}}, E$ is the Young's modulus and $\nu$ is the Poisson's ratio as previously defined. 
In our application, we only observe displacements near the surface, that is, the displacement in $z$-direction is 0 . Further, we can safely assume that the force field is parallel to the surface leading to $f_{z}=0$. Therefore, the Boussinesq-solution simplifies to

$$
\int_{\mathbb{R}^{2}} \mathbf{G}\left(\mathbf{x}^{\prime}-\mathbf{x}\right) f\left(\mathbf{x}^{\prime}\right) d \mathbf{x}^{\prime}=\mathbf{u}(\mathbf{x}) .
$$

where the Green's function reduces to

$$
\mathbf{G}(\mathbf{x})=\frac{1+\nu}{\pi E r^{3}}\left(\begin{array}{cc}
(1-\nu) r^{2}+\nu x^{2} & \nu x y \\
\nu x y & (1-\nu) r^{2}+\nu y^{2}
\end{array}\right)
$$

This is sufficient for our purposes because vertical displacements are not measured. Later, instead of calculating the displacement or deformation of the substrate from the force, we measure the displacement to calculate the force distribution. In particular, the vectorial forces, given in units of $\mathrm{Pa}$, are hence called traction forces.

\subsection{Force Dipole}

Using the elasticity theory, we now derive a relation between the forces applied to an elastic substrate and the resulting displacements. As we are studying a two-dimensional problem, we can further define a force distribution, i.e. how the single forces are placed in space. Depending on the alignment between them, one can distinguish between isotropically and anisotropically contracting cells. An isotropic contraction here corresponds to a regular or symmetric force pattern such as a contractile ring or force spots of equal magnitude distributed on regular polygons (compare Fig. 3.2). Is it possible to define a physical quantity that describes the degree of isotropy?

Such a quantity can be derived by using the so called elastic or force dipole, in analogy to the electromagnetic dipole $[56,104,126]$. The dipole is defined as a tensor $\mathbf{P}$ given by

$$
\mathbf{P}=\int \mathbf{x} \cdot \mathbf{f}(\mathbf{x}) d \mathbf{x}
$$

where $\mathbf{x}$ is a position vector and $\mathbf{f}(\mathbf{x})$ is the corresponding force density at this position. In particular, the force density here is identical to the traction force at the given position. The existance of a monopole is not considered here for a 
A

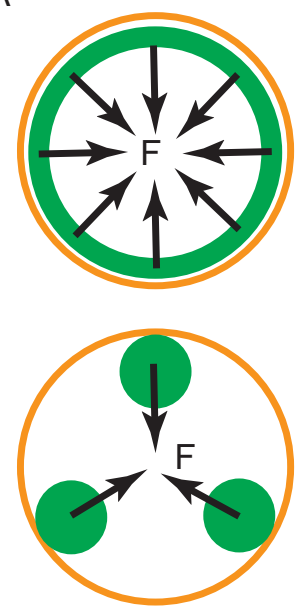

B

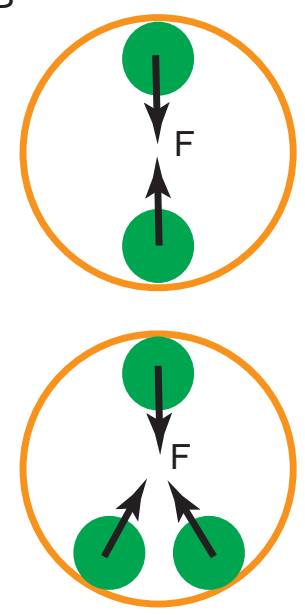

Figure 3.2.: Schematic examples of isotropically (A) and anisotropically (B) contracting cells. The orange circle denotes the cell outline and the green areas the force foot prints (areas where forces are detected through deformation of the substrate). Here, all forces (black arrows) are assumed to be of equal magnitude. The patterns in $\boldsymbol{A}$ show a radially symmetric distribution, a sign for isotropy.

The patterns in $\boldsymbol{B}$ are more polarised, a sign of anisotropy.

reason. A monopole corresponds to the vector sum of all traction forces. However, as we consider adherent, non-motile cells, the overall force is always zero. Hence, we only need to take the dipole into account.

Let us briefly consider the position vector. The vector describes the distance between the studied point and the origin of the system. At a first glance, it might appear as if the result of Eq. (3.14) depends on the choice of the coordinate system, in particular the choice of origin. So where should we define the origin in the image of a substrate deformed due to a contractile cell? Indeed, it can be shown that the choice of origin is irrelevant here. Due to the fact that we assume a net force of 0 , the choice of origin does not play a role in the result. If it can be shown that this is not the case for a given data set, there must exist a monopole, $i$. e., the cell is moving.

In our case, we only consider a two-dimensional case, $i$. e. we assume a purely planar contraction. Hence, the tensor $\mathbf{P}$ is a 2 -by-2 matrix. The integral in Eq. (3.14) can be approximated by the trapezoidal rule such that

$$
\mathbf{P}=\left[\begin{array}{ll}
x F_{x} & x F_{y} \\
y F_{x} & y F_{y}
\end{array}\right]
$$


where $F_{i}$ is the $i$-component of the force in Newton and $i F_{j}$ in the matrix always denotes the sum over all components of the structure $i \cdot F_{j}$. As can be seen, the unit of the dipole is $\mathrm{J}$.

For a purely contractile cell that is not rotating or moving, $\mathbf{P}$ is symmetric and the eigenvectors determine the direction of the major and minor dipole axis. The corresponding eigenvalues give the magnitude of the major $\left(D_{1}\right)$ and minor $\left(D_{2}\right)$ dipole. In particular, negative eigenvalues describe a contracting system while positive eigenvalues are equivalent to a dilating system. Due to their mathematical definition, the axes of the dipoles are always perpendicular to each other. For an isotropic contraction, e.g. a perfect contractile circle, both axes have the same magnitude. For increasing anisotropy, the quotient or ratio between them progressively deviates from 1 . Hence, coming back to our original question, the degree of isotropy can be defined by the ratio $q_{\text {dipole }}$ given by

$$
q_{\text {dipole }}=\frac{D_{1}}{D_{2}}
$$

Note that $q \in[1, \infty)$.

\subsection{Fluid Dynamics}

For the second set of experiments, we include a fluid flow within a channel to the force measurements of the platelets. The analysis of the forces exerted by the cells is conducted with the theory described above. However, to be able to design our flow chamber, we first need to understand the physical concepts that are of relevance in our set-up. This means we have to understand the fluid motion within a channel as well as the mass transport and diffusion under flow. In particular, we here use the concepts of microfluidics, the study of flow on small length scales. Briefly, for our specific problem, our microfluidic unit is a combination of two different channels connected in series, sketched in Fig. 3.3 A. In one channel, a substrate is embedded on which the platelets are able to attach and spread, enabling us to measure the exerted forces, compare Fig. 3.3 B and C. This chamber mimics the "blood vessel". In an additional channel preceding the measuring chamber, the platelets are mixed with the trigger substance thrombin in a controlled fashion. This helps to avoid pre-mature triggering within a shared syringe or delayed activation within the measuring chamber. 
A

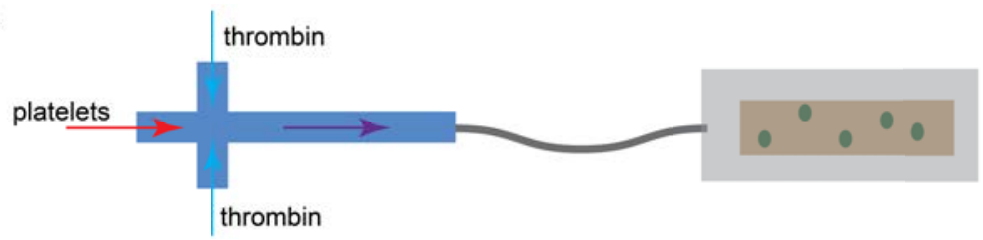

B

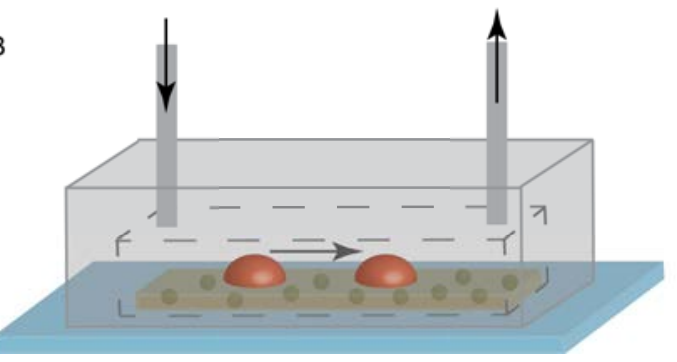

C

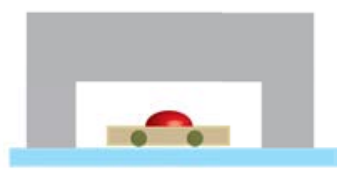

Figure 3.3.: A A sketch of the entire device designed. The platelets enter through the central inlet of the left device, thrombin is added through the side inlets. The mixing channel is connected through a connecting tube (dark grey) to the measuring chamber (light grey), containing a PAA substrate (brown with green beads). The measuring chamber is sketched in profile in $\boldsymbol{B}$. On a glass slide (light blue), a PAA substrate stripe (brown) containing fluorescent beads (green) is situated. Above it, a microfluidic chamber is build from PDMS (gray, channel denoted with dashed lines). On the top, tubing is inserted into the PDMS, acting as inlet and outlet. On the gel, coated with fibrinogen, platelets can adhere (red). C A cross-section through the channel. This figure is partially taken from Ref. [37]. 


\subsubsection{Fluid Flow in Microfluidic Channels}

First, we study the fluid flow or fluid motion within an arbitrary channel. The fundamental equation describing the flow of incompressible Newtonian fluids is the so called Navier-Stokes equation given as

$$
\rho\left(\frac{\partial \mathbf{v}}{\partial t}+(\mathbf{v} \cdot \nabla) \mathbf{v}\right)=-\nabla p+\eta \nabla^{2} \mathbf{v}+\mathbf{f}_{\text {ext }}
$$

subject to the incompressibility condition $\nabla \cdot \mathbf{v}[44]$. Here, $\rho$ denotes the density, $p$ the pressure, $\eta$ the viscosity, $\mathbf{f}_{\text {ext }}$ the external forces and $\mathbf{v}$ and $t$ as previously the velocity and time, respectively. On the left hand side of the equation, the single terms correspond to the fluid acceleration and the convection, representing the inertial forces. On the right hand side, the terms correspond to the pressure gradient and the viscous forces.

Depending on whether the viscous or inertial forces dominate the flow process, different phenomena within the fluid are observed such as laminar flow or the presence of turbulences. The relationship between these forces is characterised by the Reynolds number

$$
R e:=\frac{\rho v D_{H}}{\eta},
$$

where $D_{H}$ denotes the hydraulic diameter. Note that the Reynolds number is dimensionless. For $R e>2300$, turbulent flow may be observed while lower values indicate laminar flow [6].

From the velocity field of a Newtonian fluid, several other physical quantities can be derived. One of them is the shear rate, $\gamma$, which describes how one layer of fluid passes over a neighbouring fluid layer. Hence, it is proportional to the gradient of the velocity $\mathbf{v}$

$$
\gamma=\nabla \mathbf{v}+(\nabla \mathbf{v})^{T}
$$

and is measured in reciprocal seconds, $\mathrm{s}^{-1}$ [44]. We define the scalar norm of the shear rate as $\gamma=\sqrt{\frac{1}{2} \gamma: \gamma}$ such that $\mathbf{a}: \mathbf{b}=\sum_{n} \sum_{m} a_{n m} b_{n m}$ [15]. Further, from the shear rate, we can calculate the shear stress, $\tau$. The shear stress represents, analogous to the shear rate, the frictional forces between fluid layers [44]. Simultaneously, if a body is inserted within the fluid flow, the shear stress results in a force acting on the body to deform it as depicted in Fig. 3.1 C. For Newtonian fluids, this corresponds to the linear relationship

$$
\tau=\eta \gamma
$$


For our specific case, we study a mixed solution of buffer, platelets and thrombin. From a fluid dynamics point of view, due to the relatively low content of all other substances, this mixture may be approximated by its major component, namely water. Water is considered a Newtonian fluid, meaning we can apply Eq. (3.17) to study its behaviour. At the same time, the concept of microfluidics is used, i.e. the study of flow on small length scales. Generally, the usage of microfluidics has two major advantages. First, only limited amounts of substance is needed to measure the desired properties of the species. Second, due to the small length scales, the Reynolds numbers are often so low that a laminar flow regime is observed $[6,30]$. These facts are employed in the construction of our measuring devices. In particular, we later show that the chamber we design exhibits a Reynolds number of no more than 0.09 for the highest used flow rate during the experiments. While this is on the larger side of the Reynolds number usually associated with microfluidics (orders of $10^{-2}$ or $10^{-3}$ ), it is still fairly low. Thus, we are well within the laminar flow regime.

\subsubsection{Mass Transport in Microfluidic Channels}

Next, let us consider the mass transport within our device. In particular, as mentioned above, we want to mix a cell solution with a trigger substance within another microfluidic device. Here, we use a three inlet, one outlet device. On one hand, this device mixes the solutions as controlled as possible. At the same time, the shear rate remains reasonable low as to not trigger platelet activation which is known to happen at high shear rates.

To study the mixing of the both substances, in addition to the fluid flow as described in the previous section, we also have to consider the transport of our chemicals within the channel. Here, two processes play a role; first the active mass transport or convection due to the fluid flow and secondly the diffusion within the solution. The convection-diffusion equation for mass transfer is generally given by

$$
\frac{\partial c}{\partial t}=\nabla \cdot(D \nabla c)-\nabla \cdot(\mathbf{v} c)+R
$$

where $c$ denotes the concentration of the studied substance, $D$ its diffusion coefficient and $R$ additional sources or sinks of $c$, meaning if a substance is created or used up during the process ( [113], Chapter 3). The velocity field $\mathbf{v}$ is in our case given by Eq. (3.17). Note that the lower the diffusion coefficient is, the more the convection part dominates the behaviour of the substance. The diffusion coefficient describes the movement of the particles in flow not driven by an active mass 
transport. It was previously stated by Einstein and Smoluchowski that it is given by

$$
D=\mu k_{B} T
$$

where $T$ is the temperature, $k_{B}$ the Boltzmann constant and $\mu$ is the mobility of the studied particle or molecule. At low Reynolds numbers, so in flow regimes as studied here, the mobility is proportional to the drag coefficient $c_{d}$ such that $\mu=c_{d}^{-1}$. If we further approximate the studied particles by small spheres, it was derived by Stokes that

$$
c_{d}=6 \pi \eta r_{p},
$$

with $r_{p}$ being the radius of our particle. Hence, it follows that

$$
D=\frac{k_{B} T}{6 \pi \eta r_{p}} .
$$

For our experiments, we can directly see that the diffusion coefficient for the platelets is considerably larger than for thrombin due to their difference in size. In particular, the diffusion process of the platelets is so slow that an even concentration within the channel is not reached. For the thrombin, this is not the case and an its even distribution over the entire channel width is taken as the point of equal activation for the cells. The thorough characterisation of this process is found in the results, Section 7.3.1.

\subsection{Stresses in a Cell}

At a later time point in this work, we also consider the deformations of a cell within a flow field. This means, we wish to study the deformation of an elastic body exposed to an external force due to an applied flow field. We have previously introduced the concept of the shear stress, the stress in the flow due to the velocity gradient. At the same time, going back to Section 3.1, we saw that the stress is actually a tensor. For a three-dimensional body, the tensor is a 3 -by-3 matrix. In particular, this tensor does not only contain shear stresses but also normal stresses. Note that the shear stress from the stress acting on the matrix is generally not equivalent with the shear stress within the fluid. If we now want to study the stress that deforms our body, or here, the cell, can we define a scalar stress quantity that describes the entire stress distribution? Especially, we want to use this scalar quantity to determine the positions of highest stress, i.e. the positions at which the connection between the cell and the substrate will first break. 
Indeed, such a quantity exists, the so called von Mises stress. The von Mises stress originates from structural and material analysis where one is interested in whether an isotropic material will yield or break when exposed to an arbitrary load. Let us first define the hydrostatic stress $\sigma_{h y d}$, which relates to the normal stresses such that

$$
\sigma_{h y d}=\frac{1}{3} \sum_{i} \sigma_{i i}=\frac{1}{3} \operatorname{trace}(\boldsymbol{\sigma}) .
$$

The hydostatic stress can be written as a diagonal matrix with the scalars as entries. The deviatoric stress $\sigma_{d e v}$ is the remaining stress tensor

$$
\boldsymbol{\sigma}_{d e v}=\boldsymbol{\sigma}-\boldsymbol{\sigma}_{h y d}
$$

The same partition can be made for the strain tensor $\boldsymbol{\epsilon}$.

Let us now go back to Hook's law, Eq. (3.9). In tensor form, the equation is given as

$$
\epsilon_{i j}=\frac{1}{E}\left((1+\nu) \sigma_{i j}-\nu \delta_{i j} \sigma_{k k}\right)
$$

Further, it can be rewritten as

$$
\frac{1}{3} \delta_{i j} \epsilon_{k k}=\frac{1-2 \nu}{3 E} \delta_{i j} \sigma_{k k}
$$

which contains both the hydrostatic stress and strain terms. Combining Eq. (3.27) and Eq. (3.28), we get

$$
\epsilon_{i j}-\frac{1}{3} \delta_{i j} \epsilon_{k k}=\frac{1+\nu}{E}\left(\sigma_{i j}-\frac{1}{3} \delta_{i j} \sigma_{k k}\right) .
$$

Thus, we are able to also relate the deviatoric stress and strain terms. The von Mises stress is now defined as

$$
\sigma_{\text {Mises }}=\sqrt{\frac{3}{2} \sigma_{i j} \sigma_{i j}-\frac{1}{2} \sigma_{k k}^{2}}
$$

or, in purely deviatoric terms,

$$
\sigma_{\text {Mises }}=\sqrt{\frac{3}{2} \sigma_{d e v, i j} \sigma_{d e v, i j}}
$$




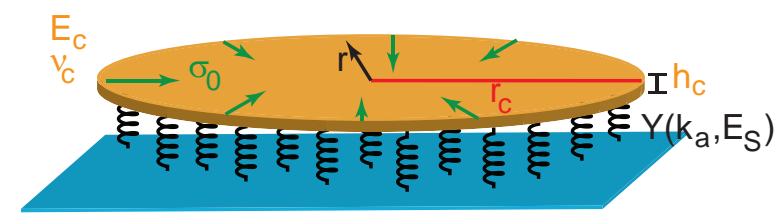

Figure 3.4.: A schematic representation of the model described in Hanke et al. [36]. The platelets is reduced to a circular disc (orange) of radius $r_{c}$ (red) and height $h_{c}$. The platelet is characterised further by the elastic modulus $E_{c}$ and Poisson's ratio $\nu_{c}$. The active stress of the platelet during contraction is given by $\sigma_{0}$. The combined stiffness density of the anchor proteins and substrate is given as $Y$ (black), a function of the single stiffness components $k_{a}$ (protein) and $E_{S}$ (substrate). As the problem is axially symmetric, the independent variable is denoted by $r$.

Hence, the von Mises stress is related to the shear stress but a scalar quantity. As previously mentioned, the von Mises stress originates from material science. In particular, if we know the strength of our material, say an anchor protein of our cell to the substrate, we are able to determine at which external load our cell would detach.

\subsection{Modelling a Contractile Cell}

For the interpretation of our data, we model the contracting cell on the elastic substrate. In particular, we are interested in the interaction between the cell and the stiffness of the substrate. Here, different models exist, varying in complexity. For our specific problem, we employ the model introduced by Edwards et al. [26]. A sketch of the model can be found in Fig. 3.4. Unless declared otherwise, the same variable notation holds as used in Section 3.1. The entire derivation for our specific problem of a contractile blood platelet, i.e. the deviations from the original works by Edwards et al. [26], can be found in Hanke et al. [36]. ${ }^{1}$

The original model [26] was derived to analytically solve the problem of a cell layer contracting on a set of micro-pillars. Here, the micro-pillars are described as a set of springs with a given spring constant or stiffness. We, however, study a single platelet contracting on an elastic substrate. Thus, to mathematically describe the interactions between cell and substrate, we define an elastic string stiffness density $Y$ to reflect both the stiffness of the substrate as well as the anchor

\footnotetext{
${ }^{1} \mathrm{I}$ am indepted to D. Probst, U. Schwarz (University of Heidelberg) and A. Zemel (Hebrew University of Jerusalem) for providing theses derivations.
} 
proteins between cell body and substrate. Additionally, instead of studying a cell layer, our object is a single platelet approximated by a contractile disc situated on the springs.

Next, let us define a number of physical properties of the blood platelet. First, for the elastic platelet, we define the elastic modulus $E_{c}$ as well as a Poisson's ratio $\nu_{c}$. Furthermore, as we approximate our platelet as a contractile disc, we set the radius to $r_{c}$ and the height to $h_{c}$. Lastly, the active stress of the platelet is set to $\sigma_{0}$. The force balance is then given by

$$
\nabla \boldsymbol{\sigma}=\frac{Y}{h_{c}} \mathbf{u}
$$

We again assume small displacements within the deformed layer as done previously in Section 3.1, which allows us to linearise the strain. Then, the single stress' components take the form

$$
h_{c} \sigma_{i j}=2 \epsilon_{i j} \frac{h_{c} E_{c}}{2\left(1+\nu_{c}\right)}+\left(\frac{h_{c} E_{c} \nu_{c}}{1-\nu_{c}^{2}} \epsilon_{k k}+\sigma_{0} h_{c}\right) \delta_{i j}
$$

where $i, j, k$ denote the three dimensions. Let us denote $\lambda=\frac{h_{c} E_{c} \nu}{1-\nu^{2}}$ and $\mu=\frac{h_{c} E_{c}}{2\left(1+\nu_{c}\right)}$. We can further simplify Eq. (3.33) if one assumes that the active stress within the platelet is constant at each point, hence, its derivative in space is 0 . The force balance is now given by

$$
h_{c} \frac{\partial \sigma_{i j}}{\partial x_{j}}=\lambda \frac{\partial u_{k}}{\partial x_{k i}}+\mu\left(\frac{\partial u_{i}}{\partial x_{j j}}+\frac{\partial u_{j}}{\partial x_{i j}}\right)=Y u_{i} .
$$

We then define a localisation length $l_{L}$ such that

$$
l_{L}=\sqrt{\frac{h_{c} E_{c}}{Y\left(1-\nu_{c}^{2}\right)}} .
$$

This length describes the decay of a point force as seen by the deformation of the substrate. If we now decouple the assembled spring constant $Y$ into its two components, namely the stiffness of the substrate as well as the protein bonds as previously demonstrated in Ref. [5], we re-write Eq. (3.35) as

$$
l_{L}=\sqrt{\frac{h_{c} E_{c} L^{2}}{k_{a} N_{a}}+\frac{h_{c} E_{c}}{\pi E_{s}}\left(\frac{1}{2 \pi h_{s}\left(1+\nu_{s}\right)}+\frac{1}{L}\right)^{-1}}
$$


with $N_{a} / L^{2}$ being the ligand density and $k_{a}$ its stiffness, $E_{s}$ the elastic modulus of the substrate, $\nu_{s}$ its Poisson's ratio and $h_{s}$ its height. We combine the properties of the adhesion region, i.e. the layer of the ligands, by setting $\frac{N_{a} k_{a}}{L^{2}}$ as the so called adhesion layer stiffness density.

Let us now take a look at the displacement we observe in the substrate. As our cell is modelled as a circular disc of isotropic contraction, in mathematical terms, the problem is axially symmetric and the strain tensor is given in spherical coordinates as

$$
r^{2} \frac{d^{2} u}{d r^{2}}+r \frac{d u}{d r}-\left(1+\frac{r^{2}}{l_{L}^{2}}\right) u=0,
$$

with $u$ being the displacement as before. Note that due to the condition of isotropy, the angle does not play a role in this equation. We further have the boundary condition $u(r=0)=0$ and

$$
\frac{\partial u}{\partial r}+\frac{\lambda}{\lambda+2 \mu} \frac{u}{r}=-\frac{\sigma_{0}}{\lambda+2 \mu}
$$

at $r=r_{0}$. The solution is then given by

$$
u(r)=-l_{L} \frac{\sigma_{0}}{\lambda+2 \mu} \cdot \frac{I_{1}\left(\frac{r}{l_{L}}\right)}{I_{0}\left(\frac{r_{c}}{l_{L}}\right)-\frac{2 \mu}{\lambda+2 \mu} \frac{l_{L}}{r_{c}} I_{1}\left(\frac{r_{c}}{l_{L}}\right)}
$$

with $I_{0}$ and $I_{1}$ being the modified Bessel functions of first kind. The total force is given by

$$
F=\int Y|u| d \mathbf{x}
$$

Evaluating this integral, we obtain for the theoretical total force the expression

$$
F_{\text {theo }}=\pi^{2} r_{c} h_{c} \sigma_{0} \frac{I_{1}\left(\frac{r_{c}}{l_{L}}\right) L_{0}\left(\frac{r_{c}}{l_{L}}\right)-I_{0}\left(\frac{r_{c}}{l_{L}}\right) L_{1}\left(\frac{r_{c}}{l_{L}}\right)}{I_{0}\left(\frac{r_{c}}{l_{L}}\right)-\left(1-\nu_{c}\right) \cdot \frac{l_{L}}{r_{c}} \cdot I_{1}\left(\frac{r_{c}}{l_{L}}\right)} .
$$

Here, $L_{n}(x)$ denotes the modified Struve function. From this relation, we can determine the behaviour of the total force in its limiting cases. The first limit is the case of soft substrates. As $L_{0}(0)=L_{1}(0)=0$ and the localisation length increases towards infinity, the total force goes to 0 . On stiff substrates, however, the localisation length decreases to its minimal value which in turn increases the total force to its maximal value. Note that the minimal localisation length is given by the expression $l_{L, \text { min }}=\sqrt{\frac{h_{c} E_{c} L^{2}}{N_{a} k_{a}}}$. Hence, $l_{L}$ and thus the maximal theoretical total force is governed by two entities, one stemming from the cell properties $\left(E_{c} h_{c}\right)$ and one stemming from the adhesion layer $\left(\frac{N_{a} k_{a}}{L^{2}}\right)$. 
Lastly, let us find a relationship between the total force and the cell radius. We approximate the Bessel functions given in Eq. (3.41) for $x>>n$ by the expression $I_{n}(x) \approx \frac{e^{x}}{\sqrt{2 \pi x}}$. This yields for $F_{\text {theo }}\left(r_{c}\right)$ that

$$
\frac{F_{\text {theo }}}{2 \pi r_{c}} \approx \sigma_{0} h_{c}\left(1+\left(\frac{1}{2}-\nu_{c}\right) \frac{l_{L}}{r_{c}}+O\left(\frac{l_{L}}{r_{c}}\right)^{2}\right) .
$$

From here, we can directly see that, if $r_{c} \gg l_{L}$, it follows that $\frac{F_{\text {theo }}}{2 \pi r_{c} h_{c}}=\sigma_{0}$. 


\section{Materials and Methods}

In the present chapter, all methods used for the experiments are summarised. In Section 4.1, the isolation of platelets from concentrates is described, followed by the fabrication of the PAA gels used in all experiments in Section 4.2. The assembly of the different microfluidic devices is outlined in Section 4.3. Finally, the recording of the data is explained in Section 4.4. Note that Sections 4.1, 4.2.1 and 4.4.2 are part of Hanke et al. [36] and Sections 4.2.2, 4.3 and 4.4.3 are part of Ref. [37].

All buffers and solutions mentioned in this chapter are listed in Table 4.1 along with their chemical composition. All commercial chemicals and tools used are found in Table 4.3 along with the corresponding supplier.

\subsection{Platelet Isolation From Plasma Concentrates}

The plasma isolation was done according to Ref. [107]. The platelets were isolated from plasma concentrates donated to the University Medical Center Göttingen but expired for clinical use. Thus, platelets were used 4 to 6 days after donation. All work described below was performed in a clean bench. Before starting the isolation, at least $30 \mathrm{~min}$ in advance, the tubes containing PSG (Pipes Saline Glucose) and HT buffer (Hepes-Tyrode buffer) were pre-heated in the incubator, slightly opened. This was done to ensure that the buffers exhibited similar conditions as found in vivo, namely $5 \% \mathrm{CO}_{2}$ content and a temperature of $37^{\circ} \mathrm{C}$. When not used, the tubes were always stored in the incubator during the isolation process.

From the blood concentrates, the cap on the outlet was removed. The bag was still closed at this point as an additional, self-sealing rubber stopper was incorporated into the bag itself. A $2 \mathrm{~mL}$ syringe was connected to a needle of size 1 and carefully inserted into the rubber stopper without perforating the bag. Slowly, the syringe was filled, detached from the needle and discarded including the $2 \mathrm{~mL}$ of blood plasma. A $5 \mathrm{~mL}$ syringe was subsequently attached to the needle and filled with $4 \mathrm{~mL}$ of plasma. The syringe was again detached and the content transferred into a $15 \mathrm{~mL}$ reaction tube by slowly letting it run down the tube's wall. $90 \mu \mathrm{L}$ of PSG 
was mixed with $10 \mu \mathrm{L}$ of $\mathrm{PGE}_{1}$ (Prostaglandin $\mathrm{E}_{1}$ ) and added into the $15 \mathrm{~mL}$ tube. Mixing it shortly by swirling it gently by hand, the tube was put into the centrifuge kept at $21^{\circ} \mathrm{C}$. A counterbalance was inserted, the centrifuge was closed and set to $480 \mathrm{~g}$ for $20 \mathrm{~min}$.

After centrifuging, a pellet consisting of platelets and red blood cells were found in the bottom of the tube. The supernatant was carefully removed and $4 \mathrm{~mL}$ of PSG was added. The cells were now brought back into suspension by gently pipetting the fluid up and down until no clumps of cells were observed anymore. It is important to note that the re-suspension was done very gently as to not stress the platelets unnecessarily.

Subsequently, $10 \mu \mathrm{L} \mathrm{PGE}_{1}$ was mixed with $90 \mu \mathrm{L}$ PSG and added to the platelet suspension. After swirling the tube, it was inserted into the centrifuge and put to the previous settings. In total, the cells including the $\mathrm{PGE}_{1}$ were centrifuged and re-suspended in $4 \mathrm{~mL}$ PSG three times. During the centrifugation, in a separate reaction tube, BSA (bovine serum albumin) was added to the HT buffer to a final concentration of $5 \mathrm{mg} / \mathrm{mL}$. After the last centrifugation step, the cells were resuspended in $1 \mathrm{~mL}$ of HT-BSA buffer until the solution was a homogeneous, milky mixture. $90 \mu \mathrm{L}$ of the suspension was transferred into a small reaction tube while the larger tube was fastened into a smaller table rotator and kept in motion with $12 \mathrm{rpm}$. The cells on the rotator were later used for the experiments. To avoid aggregation due to stagnation of the solution, they were kept in constant motion.

The small amount of platelet suspension in the smaller tube was then used to determine the concentration of cells. The fluid was filled into a haematocrit capillary using capillary forces (Fig. 4.1). On the capillary, a small red ring was displayed around its circumference to mark the maximum fluid level which not to exceed. The filled capillary was sealed at the lower end using modelling clay, wrapped in a piece of low-lint tissue wipe (KimTech tissue, see Table 4.3) to avoid breakage and inserted into a $15 \mathrm{~mL}$ tube. The tube was put into the centrifuge and rotated at $1000 \mathrm{~g}$ at $21^{\circ}$ for $10 \mathrm{~min}$. The cells inside the capillary were then pelleted directly above the clay and, using a haematocrit reader chart, the total concentration of cells was determined. Typically, the concentration amounted to $4 \cdot 10^{9}$ cells $/ \mathrm{mL}$ to $6 \cdot 10^{9}$ cells $/ \mathrm{mL}$. For the experiments, a final concentration of $2 \cdot 10^{7}$ cells $/ \mathrm{mL}$ was used.

For the static experiments, the platelets were stained with a membrane dye, CellMask Deep Red dye, to identify the time point of attachment during recording. When staining the platelets, $0.5 \mu \mathrm{L}$ of dye was mixed in $999.5 \mu \mathrm{L}$ of HT-BSA buffer to a final concentration of $2.5 \mu \mathrm{g} / \mathrm{mL}$ until the solution's colour was uniform. The 


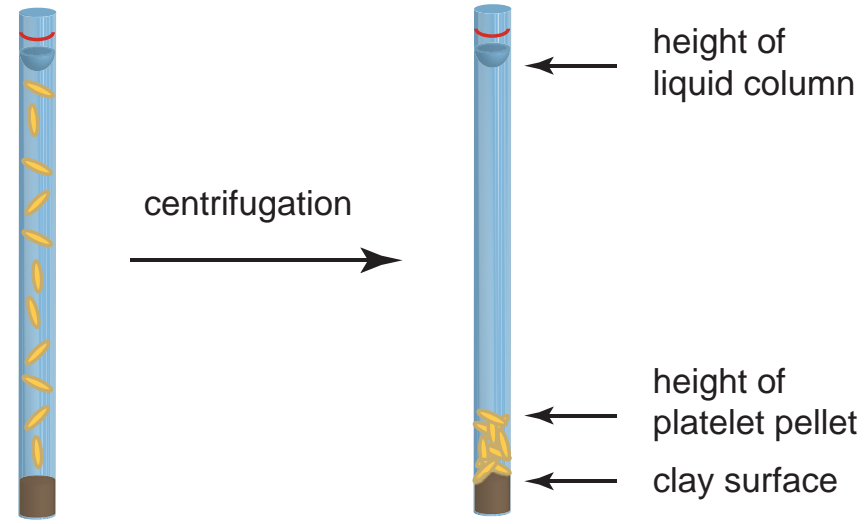

Figure 4.1.: A sketch of the process of cell counting. A capillary is filled with a suspension of platelets (yellow). The fluid upper surface should not exceed the red line at the top of the capillary. Leakage is prevented by sealing the capillary with clay at the bottom (brown). After centrifugation, the platelets are found directly above the clay. By aligning the capillary to a reader chart, the height from the upper clay surface to the upper boundary of the platelets yields the concentration of cells per millilitre.

platelets were then diluted to their final concentration in the dye containing buffer. All following steps had to be made quickly as the platelets tended to take up the dye into the cytoplasma, thus reducing the time in which reliable detection is possible. In particular, between the first staining step and the start of recording, on average, 20 min elapsed. The tube with the stained platelets was incubated for 5 min at $37^{\circ} \mathrm{C}$. Meanwhile, $10 \mu \mathrm{L} \mathrm{PGE}_{1}$ was mixed with $90 \mu \mathrm{L}$ HT-BSA buffer. $25 \mu \mathrm{L}$ of the diluted $\mathrm{PGE}_{1}$ was pipetted into the cell suspension before the tube was transferred into the centrifuge pre-set to $480 \mathrm{~g}$ for $5 \mathrm{~min}$. The supernatant was removed and the platelets re-suspended in $1 \mathrm{~mL}$ HT-BSA buffer for $8 \mathrm{~min}$ to $10 \mathrm{~min}$. Note that the cell pellet was not visible anymore at this stage. The stained platelets were then directly used for recording.

Additionally to the above steps, two further aliquots had to be prepared. One aliquot of some millilitres of HT-BSA buffer solution was prepared for the later washing of the substrates. Furthermore, thrombin solution was prepared with a concentration of $40 \mathrm{u} / \mathrm{mL}$ by mixing $25 \mu \mathrm{L}$ of the thrombin stock as denoted in Table 4.1 with $225 \mu \mathrm{L}$ HT-BSA solution. 


\begin{tabular}{|c|c|}
\hline Buffer / Solution & Chemical Composition \\
\hline APS working solution & $10 \mathrm{mg}$ APS in $100 \mu \mathrm{L}$ MilliQ water \\
\hline Fibrinogen & $100 \mathrm{mg}$ in $5 \mathrm{~mL} \mathrm{MilliQ} \mathrm{water}$ \\
\hline Fibrinogen, Alexa 488 labelled & 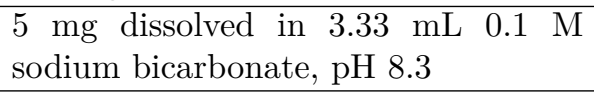 \\
\hline $\begin{array}{l}\text { fluorescent beads stock solution, } \\
40 \mathrm{~nm} \text { diameter, } 505 / 515 \mathrm{~nm} \text { excita- } \\
\text { tion/emission wavelength }\end{array}$ & $\begin{array}{l}10 \mu \mathrm{L} \text { fluorescent beads, } 5 \% \text { solids, in } \\
240 \mu \mathrm{L} \text { MilliQ water }\end{array}$ \\
\hline $\begin{array}{l}\text { fluorescent beads stock solution, } \\
100 \mathrm{~nm} \text { diameter, } 660 / 680 \mathrm{~nm} \\
\text { excitation/emission wavelength }\end{array}$ & $\begin{array}{l}10 \mu \mathrm{L} \text { fluorescent beads, } 2 \% \text { solids, in } \\
90 \mu \mathrm{L} \text { MilliQ water }\end{array}$ \\
\hline $\begin{array}{l}\text { fluorescent beads stock solution, } \\
200 \mathrm{~nm} \text { diameter, } 660 / 680 \mathrm{~nm} \\
\text { excitation/emission wavelength }\end{array}$ & $\begin{array}{l}10 \mu \mathrm{L} \text { fluorescent beads, } 2 \% \text { solids, in } \\
90 \mu \mathrm{L} \text { MilliQ water }\end{array}$ \\
\hline Hepes buffer, $0.5 \mathrm{M}$, pH 8.0 & $\begin{array}{l}119.15 \mathrm{~g} \text { Hepes in } 1 \mathrm{~L} \text { sterile distilled } \\
\text { water; working solution } 50 \mathrm{mM}\end{array}$ \\
\hline Hepes-Tyrode buffer, pH 7.4 & $\begin{array}{l}134 \mathrm{mM} \mathrm{NaCl}, 12 \mathrm{mM} \mathrm{NaHCO}_{3} \text {, } \\
2.9 \mathrm{mM} \mathrm{KCl}, 1 \mathrm{mM} \mathrm{MgCl} 2,5 \mathrm{mM} \\
\mathrm{HEPES}, 5 \mathrm{mM} \text { glucose, 0.34 mM } \\
\mathrm{Na}_{2} \mathrm{HPO}_{4}\end{array}$ \\
\hline PBS, 10X, pH 7.2 & $\begin{array}{l}1.37 \mathrm{M} \mathrm{NaCl}, 27 \mathrm{mM} \mathrm{KCl}, 43 \mathrm{mM} \\
\mathrm{Na}_{2} \mathrm{HPO}_{4}{ }^{*} 12 \mathrm{H}_{2} \mathrm{O}, 14 \mathrm{mM} \mathrm{KH} \mathrm{PO}_{4} \\
\text { working solution } 1 \mathrm{X}\end{array}$ \\
\hline PBS-Glutaraldehyde, $0.5 \%$ & $\begin{array}{l}357 \mu \mathrm{L} 70 \% \text { glutaraldehyde in } 50 \mathrm{~mL} \\
1 \mathrm{X} \text { PBS }\end{array}$ \\
\hline Pipes Saline Glucose (PSG), pH 6.8 & $\begin{array}{l}5 \mathrm{mM} \text { PIPES, } 145 \mathrm{mM} \mathrm{NaCl}, 4 \mathrm{mM} \\
\mathrm{KCl}, 1 \mathrm{mM} \mathrm{MgCl}_{2} * 6 \mathrm{H}_{2} \mathrm{O}, 5 \mathrm{mM} \text { glu- } \\
\text { cose, } 0.05 \mathrm{mM} \mathrm{Na}_{2} \mathrm{HPO}_{4}\end{array}$ \\
\hline Prostaglandin $\mathrm{E}_{1}\left(\mathrm{PGE}_{1}\right)$ & $1 \mathrm{mg} \mathrm{PGE}{ }_{1}$ in $940 \mu \mathrm{L}$ DMSO \\
\hline Sulfo-SANPAH & $50 \mathrm{mg}$ in $250 \mathrm{ml}$ of $50 \mathrm{mM}$ Hepes buffer \\
\hline Thrombin & $\begin{array}{l}1 \mathrm{mg} \text { thrombin }(1 \mathrm{kU} / \mathrm{mL}) \text { in } 2.5 \mathrm{~mL} \\
\text { BSA stock solution }\end{array}$ \\
\hline
\end{tabular}

Table 4.1.: Composition of various buffers and solutions used during all experiments. 


\subsection{Fabrication of PAA Gels}

For all tTFM measurements in this thesis, PAA gels were used whose production was to some extend based on Ref [107]. In general, the gels were all fabricated similar to each other, however, some aspects differ slightly depending on the later usage. Below, the polymerisation of the gels is first described for the static experiments, followed by changes needed when conducting flow experiments.

\subsubsection{Substrates for Static Experiments}

For the static experiments, we produced comparatively large gels to increase the probability of finding a spot where the platelets attach while the substrate remained as flat as possible at a certain height. We used two different glass slides for the polymerisation, a 24-by-24 $\mathrm{mm}$ square glass slide on which we bound the gel and a $18 \mathrm{~mm}$ round cover slip to shape the gel.

To start, all glass slides were cleaned with iso-propanol and dried with nitrogen gas. For all following steps, the cover slips were kept in cleaned petri-dishes for convenience. Furthermore, henceforth, all work was done underneath the fume hood for safety reasons as several of the following substances emitted toxic fumes.

To facilitate that the gel was able to bind covalently to the glass slide, the surface was pre-coated. The chemical reactions can be found in Fig. 4.2. To start, the glass was wetted with $0.1 \mathrm{M} \mathrm{NaOH}$ using a cotton swap and left to dry. Subsequently, APTMS (3-aminopropyltrimethoxysilane) was applied, again with a cotton swap, and incubated for 5 min (panel A). Excess APTMS was washed away with MilliQ (purified) water until no white residual crystals were visible anymore. Larger remaining water droplets were removed with a low-lint wipe without touching the glass slide itself while the residual water was air dried. Due to the reaction of the silicon oxide in the glass and the silane, a $\mathrm{Si}-\mathrm{O}-\mathrm{Si}$-bound was established on the glass surface, exposing a free amine group. Subsequently, $0.5 \%$ PBS-glutaraldehyde (compare Table 4.1) was pipetted onto the glass slides and incubated for $30 \mathrm{~min}$ (panel B). The supernatant was aspired, discarded separately and residual solution washed away with MilliQ water. The glutaraldehyde polymerised, leaving a carbon-hydroxide group of the glutaraldehyde free to react with the acrylamide (panel $\mathbf{C}$ ). The coated side of the glass was then marked and air dried before usage. 
A

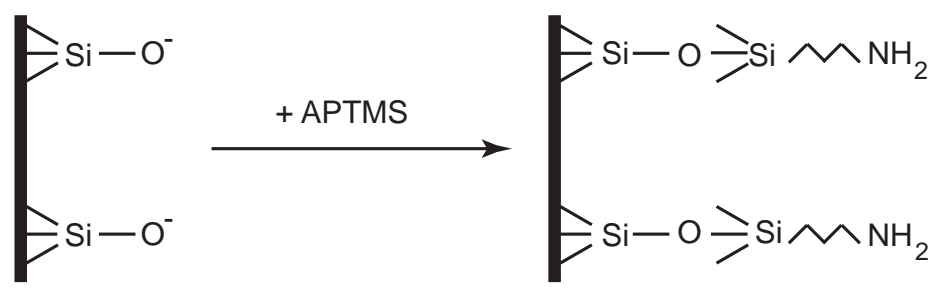

B

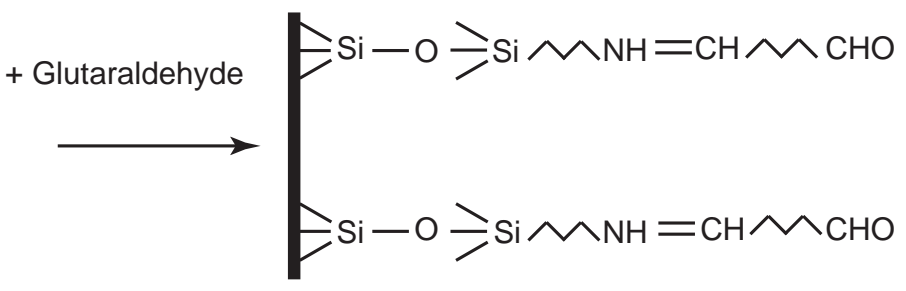

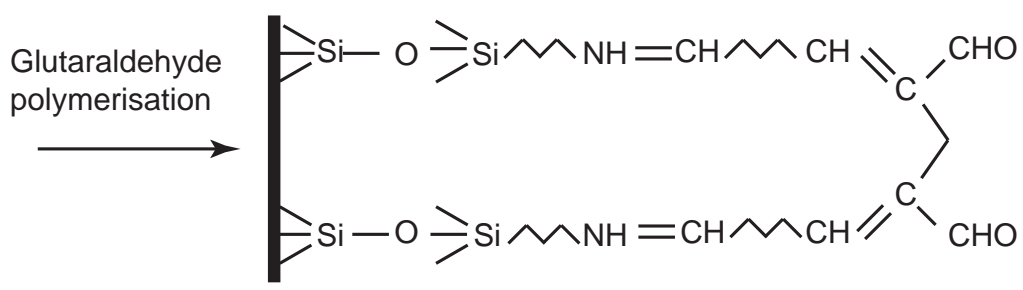

C

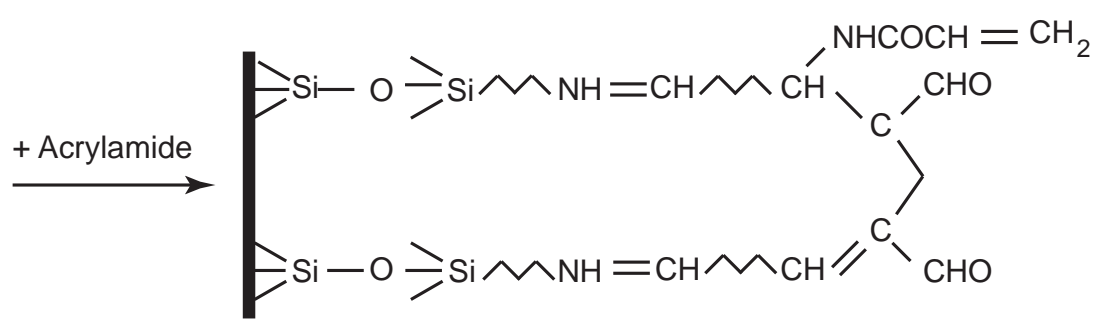

Figure 4.2.: A sketch of the binding of the PAA substrate to the glass slide. A On the glass surface, free $\mathrm{Si}-\mathrm{O}$ groups bind to the APTMS by building $\mathrm{Si}$ $\mathrm{O}-\mathrm{Si}$ bonds. $\boldsymbol{B}$ The glutaraldehyde further covalently binds to the NH group and polymerises. $\mathrm{C}$ Finally, the acrylamide binds to the $\mathrm{CH}$ group of the glutaraldehyde, facilitating the covalent attachment of the PAA substrate to the glass slide. The figure is based on Ref. [107]. 


\begin{tabular}{|c|c|c|}
\hline Elastic Modulus $[\mathrm{kPa}]$ & $40 \%$ Acrylamide $[\mathrm{mL}]$ & $2 \%$ Bis-Acrylamide $[\mathrm{mL}]$ \\
\hline \hline $19.4 \pm 0.5$ & 2 & 0.7 \\
\hline $29.3 \pm 0.5$ & 2.5 & 0.75 \\
\hline $41.2 \pm 0.9$ & 2.5 & 1.3 \\
\hline $54.1 \pm 0.7$ & 2.5 & 2.25 \\
\hline $83.1 \pm 0.3$ & 3.75 & 1.5 \\
\hline
\end{tabular}

Table 4.2.: Chemical composition of $10 \mathrm{~mL}$ PAA pre-mixed solution. Remaining volume is filled up with $1 X \mathrm{PBS}$. The elastic modulus was measured by rheology by A. Paknikar.

In parallel, the round cover slips were treated to be hydrophobic as to avoid any sticking of the gel to the glass. Here, the glass slides were wetted on both sides with Plus One Repel Silane (compare Table 4.3) and incubated for $5 \mathrm{~min}$. They were then washed with $70 \%$ ethanol followed by MilliQ water and air dried until usage.

Subsequently, all solutions needed for the PAA gel were prepared. First, the acrylamide/bis-acrylamide solutions were mixed according to Table 4.2. The catalyst APS (ammonium persulfate) was prepared from powder and kept in the fridge until usage. The stock solution of the $40 \mathrm{~nm}$ diameter fluorescent beads (Table 4.1) was homogenised by inserting the bead containing tube into the ultrasonic bath for 10 min followed by vortexing. $485 \mu \mathrm{L}$ pre-mixed PAA solution of the desired stiffness was mixed with $15 \mu \mathrm{L}$ fluorescent beads.

The next step was the polymerisation of the PAA substrates. As the polymerisation reaction was initiated as soon as the catalysts are added to the solution, it was essential to work fast to reach a good gel quality. 0.5 $\mu \mathrm{L}$ TEMED (Tetramethylethylenediamine) and $5 \mu \mathrm{L}$ APS were added into the PAA-bead-mixture and the solution mixed by pipetting up and down in between each addition. Following, $7.5 \mu \mathrm{L}$ of the final PAA solution was pipetted onto each circular cover slip. The square glass slide was carefully added onto the mixture with the coated surface facing towards the solution thus building a sandwich-like construction of glass-solution-glass (compare Fig. 4.3). The gel was polymerised for $1 \mathrm{~h}$ on a flat surface upside-down to ensure that as many beads as possible were found on the upper part of the finished gel. To avoid photo-bleaching of the beads during polymerisation, everything was covered with aluminium foil. 
A

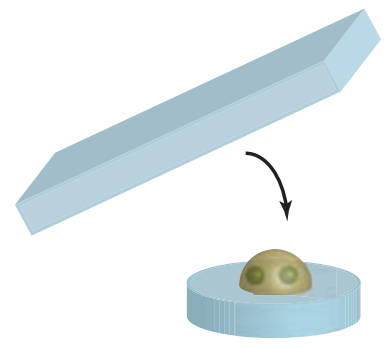

B

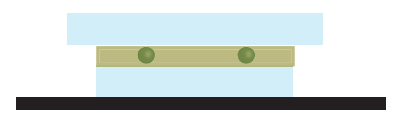

Figure 4.3.: A sketch of the assembly of the PAA substrate for static experiments. A On the circular coverslip, the acrylamide-bisacrylamide solution (brown) containing beads (green) is pipetted and gently flattened with the larger, rectangular glass slide. $\boldsymbol{B}$ The substrate is left to polymerise in an upside-down position on a flat surface (black).

After polymerisation, the glass sandwiches were immersed into 1X PBS inside a petri-dish with the correct orientation, the circular glass slide facing up. The upper glass started to detach and was then be removed carefully with a pair of tweezers. The gels were then washed in PBS to remove residual, unpolymerised PAA solution. From this point onwards, the gels were kept moist at all times.

Lastly, the gels were coated with a layer of fibrinogen to facilitate the attachment of the platelets. This was done by first binding a cross-linker, Sulfo-SANPAH (Table 4.3), onto the substrate. As Sulfo-SANPAH is a cross-linker activated by UV light and light-sensitive, the following was done with as low room illumination as possible, avoiding direct light completely. The substrates were covered with Sulfo-SANPAH and incubated under UV light, wavelength $366 \mathrm{~nm}$, for $8 \mathrm{~min}$. Afterwards, the gels were washed in Hepes buffer. This procedure was then repeated once more before the fibrinogen was added onto the substrates. For all static experiments, unlabelled fibrinogen was used. Here, $5 \mu \mathrm{L}$ fibrinogen was mixed in $995 \mu \mathrm{L} 1 \mathrm{X}$ PBS to a final concentration of $0.1 \mathrm{mg} / \mathrm{mL}$ and added to gels to cover them entirely. The substrates were incubated at $4^{\circ} \mathrm{C}$ over night. The following day, the gels were washed again in PBS and stored in PBS until usage. The finished gels were used for one week after which they started to loose their flat appearance. 


\subsubsection{Substrates for Flow Experiments}

For the flow experiments, the geometry of the gel needed to be changed entirely. As we wanted to embed the gel within a microfluidic channel, we were now in need of a thin strip of PAA gel to fit within the channel structure. This accounted for the major differences in the substrate fabrication protocol compared to the above description.

For the glass slide to which the gel was bound, rectangular glass slides of a size 24by-60 $\mathrm{mm}$ were chosen. For the glass slide which was used to flatten the substrate into its final shape, another 24-by-60 mm glass was cut with a diamond cutter into stripes of 3-by-24 mm size. The different glass slides were then treated as previously described, the larger slide with the APTMS-glutaraldehyde mixture, the smaller coverslip with Repel Silane.

As we were only interested in the influence of the shear stress on the platelets during the experiments, the stiffness of the substrates was kept constant. For all experiments, a stiffness of $41 \mathrm{kPa}$ was chosen. This stiffness fell into the middle of the elasticity range we studied in the static case. At the same time, we were sure that the substrate was stiff enough so that deformations due to the fluid flow did not occur.

To ensure that the gels were of comparable height as the substrates used in the stationary case, only $2.16 \mu \mathrm{L}$ of the finished PAA solution containing both catalysts was used. Due to the fact that the size difference between the glass slides used was comparably high, the polymerisation set-up in an upside-down manner on a flat surface as used previously did not work here. Using the previous approach, the non-polymerised solution ran a risk of being pressed out from between the glass slides, resulting in too thin substrates of about $10 \mu \mathrm{m}$ or less in thickness. However, it was still necessary to polymerise the gel upside-down to guarantee that a densely seeded bead pattern was found at the upper layer. Thus, instead of laying the ensemble on a flat working desk, a stack of thick microscopy slides were arranged such that their distance was between $24 \mathrm{~mm}$ and $55 \mathrm{~mm}$. The PAA solution was then pipetted onto the smaller coverslip and the larger glass slide with the coated side facing the smaller glass was brought into contact with the solution (Fig 4.4 A). Important at this step was to not let the larger glass fall onto the smaller glass slide but instead use capillary forces to distribute the fluid solution between both glass slides. The ensemble was then picked up and positioned on the microscopy slide stacks such that the area containing the substrate was hanging 
A

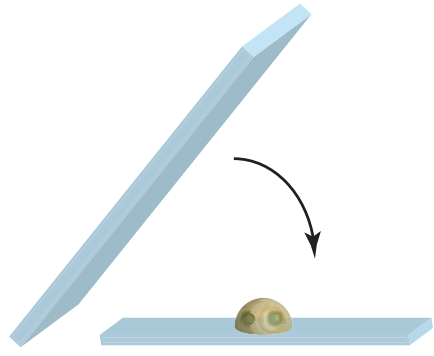

B

Figure 4.4.: The assembly of the glass-PAA-glass sandwich for flow experiments. A On the small glass stripe, the liquid PAA solution containing fluorescent beads is added. The larger glass slide is lowered carefully onto the fluid without pressing (black arrow). When the solution is spread entirely between the glass slides, the assembly is lifted up. $\boldsymbol{B}$ For the polymerisation, the assembly is left turned upside-down. To avoid pressure on the smaller glass slide, the glass sandwich is hung up between two thicker microscopy slides so the side of polymerisation is suspended in air.

upside-down between the slides (Fig 4.4 B). In this position, the set-up polymerised for one hour under aluminium foil. Afterwards, the gels were again coated with fibrinogen as described above.

For some test experiments where the attachment of fibrinogen was studied, Alexa 488 labelled fibrinogen was used. To ensure the same concentration as in the unlabelled case, $66.66 \mu \mathrm{L}$ labelled fibrinogen was mixed in $933.34 \mu \mathrm{L}$ PBS and added to the substrates. As the emission spectrum of Alexa 488 was the same as that for the normally used green beads, red fluorescent beads of various sizes as found in Table 4.3 were added for orientation.

\subsection{Assembly of Microfluidic Channels and Chambers}

In the following, the construction of the microfluidic devices used in the flow experiments were described. As already shown in Fig. 3.3, the final set-up contained two different devices connected in series. The first one was the three inlet channel to mix the thrombin and platelet suspension to reach the desired concentrations for each substance as well as the final combined flow rate. This device is hence called mixing channel to distinguish it from the second device. The latter was used as the container in which the actual measurement is conducted and is hence 


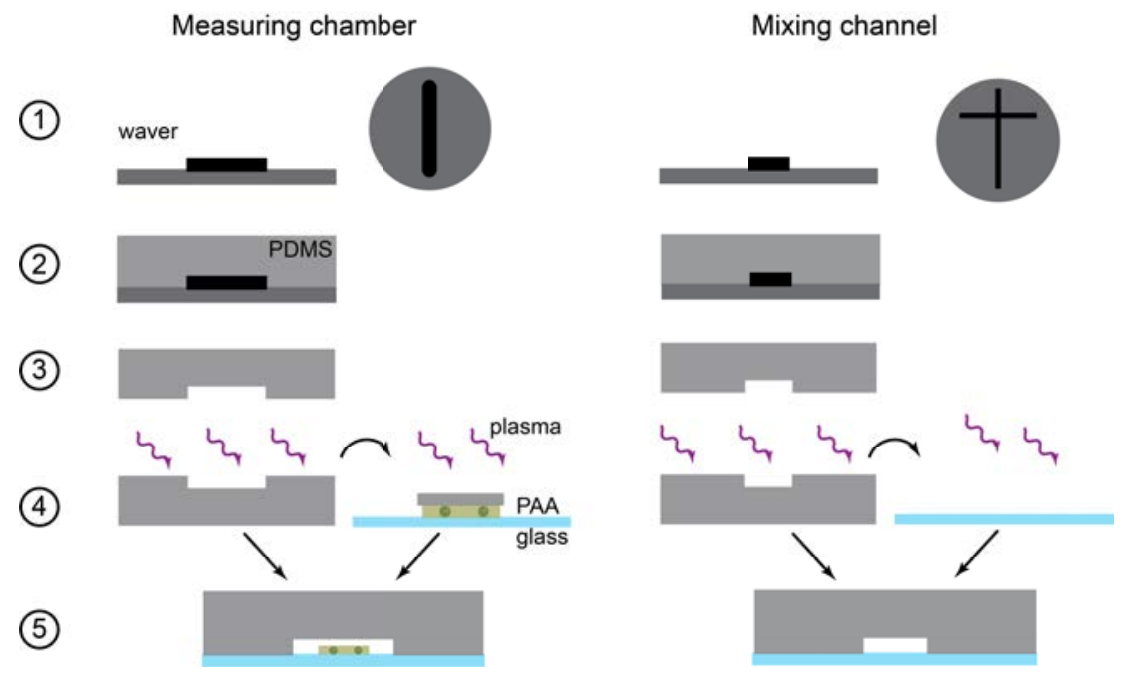

Figure 4.5.: To shape the devices, silicon wavers (dark grey) are produced, displaying the negative of the channel structures (step 1). The measuring chamber is a long, thicker channel with rounded endings and the mixing channel resembles a cross (geometries in top view shown on the right). On the waver, PDMS is cast (light gray, step 2), building the upper part of the devices upon removal from the waver (step 3). To bind the devices, the casts are plasma treated, inserted into the plasma cleaner as depicted (step 4). Together with the PDMS, the glass slides (blue) to close the channels are also plasma treated. For the measuring chamber, the glass slide already contains the PAA stripe, which is protected with a small piece of PDMS during treatment. Finally, the PDMS is bound to the glass slide (step 5). The PDMS stripe used to protect the PAA substrate is removed beforehand. The sketch is adapted from Hanke et al. [37].

called measuring chamber. The simulations conducted to characterise the devices are found in Section 7.3.1. For the following description of the fabrication and assembling of both devices, the reader is referred to Fig 4.5.

\subsubsection{Assembly of Mixing Channels}

The silicon wavers used in this section were made by photo lithography $[24,76]$. They were produced in the clean room of the Physics department by G. Brehm and E. Perego.

Here, we started out with the silicon wavers containing the negative of our desired three inlet channel structure (step 1). The wavers were cleaned by washing them with iso-propanol and blow drying them with nitrogen. Furthermore, glass slides 
with a diameter of $50 \mathrm{~mm}$ were also cleaned with iso-propanol and blown dry with nitrogen. To ensure that all liquid evaporated, the glass slides were then put on a heating plate at $90^{\circ} \mathrm{C}$.

Subsequently, PDMS was mixed thoroughly with its cross-linker in a ratio of 10:1. The PDMS was then poured onto the wavers until an average height of about half a centimetre was reached. All filled wavers were put into a desiccator and degassed for about $15 \mathrm{~min}$. If larger air bubbles remained, this procedure was repeated. Smaller bubbles were pushed away with a small pipette tip. It was important that no air remained directly on or near the channel structure. The PDMS was hardened into a rubber-like polymer by baking at $65^{\circ} \mathrm{C}$ for an hour (step 2).

Using a scalpel, the PDMS was carefully cut out from the waver to include the entire channel structure plus some additional bordering region for binding (step 3 ). To extract the final structure from the waver without damage, some isopropanol was poured onto the PDMS to build a film of liquid between the waver and PDMS. It was then removed from the waver using a pair of blunt and bended tweezers. Excess PDMS was cut with the scalpel. The in- and outlets for the tubing (polyethylen tubing Table 4.3) through which any fluid was transported later, were punched using a $0.75 \mathrm{~mm}$ biopsy puncher (Table 4.3) at the marked positions in the structure. Finally, the PDMS was cleaned again using iso-propanol and dried using first nitrogen followed by the heating plate already holding the glass slides, structure facing up (step 4).

To bind the channel structure to the glass slides, both were put on an aluminium foil covered glass pane and inserted into a plasma cleaner connected to a pump. Again, the structure in the PDMS was facing up. The air from the plasma cleaner was extracted using the pump until the plasma ignited in a purple-pink shade. The pump was disconnected from the plasma cleaner but no air injected for another $20 \mathrm{~s}$. Slowly, the air valve was opened, avoiding any strong air blasts as to not move and break the glass slide. The glass pane was moved out from the plasma cleaner and the PDMS pressed onto the glass slide with the structure facing the glass (step 5). At this point, it was important to remove all remaining air between structure and glass slide, best done by pressing softly from the centre outwards. Finally, the finished structure was again put on the heating plate for a couple of minutes before the binding was tested. If no detaching area was observed, the channel was deemed ready to use. 


\subsubsection{Assembly of Measuring Chambers}

The measuring chamber was assembled similar to the channels concerning the upper part made out of PDMS. The wavers were again fabricated in the clean room of the Physics department by E. Perego and C. Ranke.

The upper part of the channel structure was again cast from PDMS as described above (step 1 to 3). A photo is shown in Fig. 4.6 A. In- and outlets were punched with the previously used puncher of $0.75 \mathrm{~mm}$ width. Note that in this device, no mark was included as to where the holes were punched. They were however always positioned in the middle of the channel where the rectangular part of the channel transitioned into the circular closure. The PDMS was then cleaned again with iso-propanol and nitrogen and dried additionally on a heating plate at $90^{\circ} \mathrm{C}$. Now, the substrate containing glass slide was prepared. For all following steps, it was important to reduce any time periods at which the substrate was not in liquid or at least moist as the gel collapsed otherwise. The glass slide was taken out of the petri-dish containing PBS and gently washed with MilliQ water to remove residual salt crystals. All liquid on the glass slide was removed using a low-lint KimTech wipe leaving a layer of water on the substrate itself.

The glass slide was then placed onto the aluminium foil covered glass pane used inside the plasma cleaner. To protect the fibrinogen from direct exposure to the plasma and keeping the gel moist as long as possible, a thin stripe of PDMS with similar dimensions as the gel itself was gently placed onto the substrate (compare Fig. 4.6 B). Additionally, one PDMS device was put onto the glass pane with the structure facing up (step 4). The glass pane was inserted into the plasma cleaner which was then closed. To ensure that the plasma ignited even though the chamber contained small amounts of liquid, the chamber was very shortly ventilated when a pressure of about $10^{-2}$ mbar was reached. As soon as the plasma ignited in a pink-purple light, the vacuum pump was switched off. After another $10 \mathrm{~s}$, the chamber was carefully opened until it was again completely filled with air. The glass pane was taken out and the small PDMS stripe on the substrate was carefully removed with a pair of forceps.

At this point, the glass slide containing the substrate was briefly inspected. If too much PBS still remained on the glass slide, a white layer of fine salt was observed on the glass which prevented the covalent binding of the PDMS to the glass. If such a layer was seen, depending on the state of the gel, this glass slide was either discarded directly or left to soak in MilliQ water for at least $30 \mathrm{~min}$ before another attempt was made. In most cases, the border region of the gel 


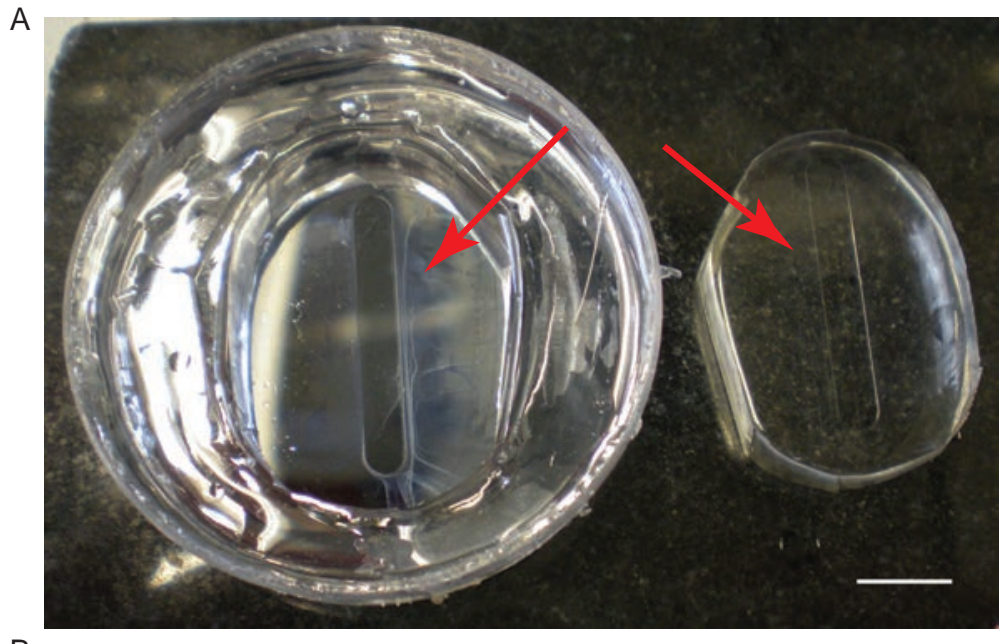

B

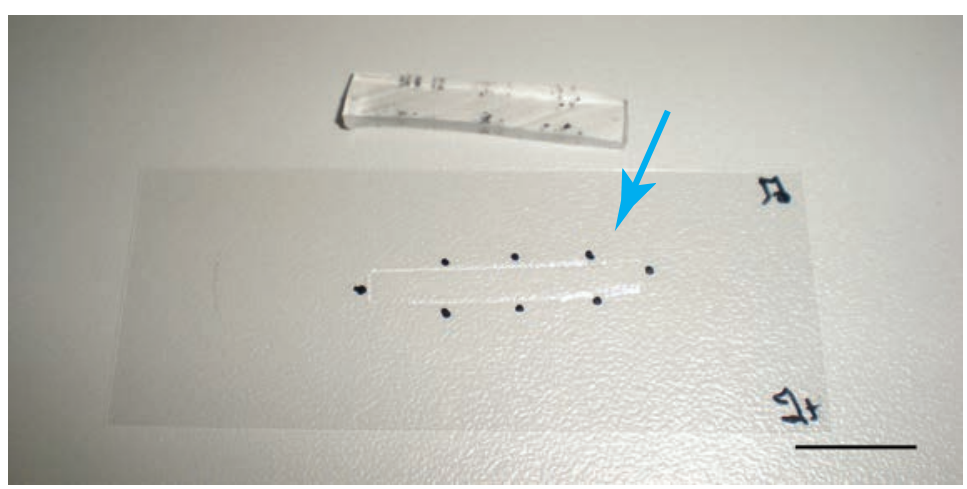

Figure 4.6.: A The PDMS cast (right) for the ceiling and side walls of the measuring chamber. On the left side of the image, the silicon waver with the chamber negative is seen. The structures are marked by a red arrow each. $\boldsymbol{B}$ The glass slide containing the PAA stripe substrate (blue arrow). The black dots mark the borders of the stripe for easier alignment after the plasma treatment. The smaller PDMS stripe at the top of the image was used to protect the substrate during plasma treatment. Scale bar: $1 \mathrm{~cm}$.

which was not protected entirely exhibited small ruffles. As long as these were confined to the outermost part of the substrate, this did not have any influence on the later measurement where only inner regions of the gel were considered. If these ruffles grew larger, parts of the gel would detach from the glass slide and could no longer be used. Lastly, if the gel became too dry in the plasma cleaner, it appeared opaque. If larger regions were affected the substrate was discarded as a full recovery of the gel was unlikely. 
If the substrate and the glass slide were deemed satisfactory, the PDMS structure was swiftly placed over the gel and pressed onto the glass from the middle outwards, avoiding any part of the substrate to be under the PDMS itself (step 5). Tubing was inserted into the holes for the in- and outlets, each of a length between $15 \mathrm{~cm}$ and $20 \mathrm{~cm}$. In some cases, during punching, small fissures arose where we ran the risk of leakage when the device was exposed to higher flow rates for several hours. To avoid leakage and ensuring that the tubing did not detach during measuring, small quantities of two component glue (UHU Plus, see Table 4.3) was added directly around the inlets and left to dry.

As the substrates were always kept moist, the entire chamber including the tubing was now filled with MilliQ water by using an automated pump system (syringe pump LA-30, Table 4.3) at $800 \mu \mathrm{L} / \mathrm{h}$ or an average velocity of $630 \mu \mathrm{m} / \mathrm{s}$ within the chamber. This was a higher flow rate compared to that used later on in the measurements to ensure that the devices also withstood the actual experiment. During the filling of the system, all air bubbles were avoided and they were reduced by gently pressing onto the chamber where air bubbles appeared. When the entire system was filled with water, the syringe pump was switched off and both tubing ends were closed by melting the tips and pressing them together. For short term storage of no more than 3 days, the devices were put into a high beaker, the tubing pointing up and stored at $4^{\circ} \mathrm{C}$ covered in aluminium foil. For longer storage of up to one week, the whole devices including tubing were immersed into a wide beaker filled with MilliQ water. As the PDMS devices tended to float in water, the devices were kept down by small weights. Again, this version of storage was kept at $4^{\circ} \mathrm{C}$ covered in aluminium foil until usage.

\subsection{Imaging of Contractile Platelets}

\subsubsection{Fluorescence Microscopy}

All imaging performed within the scope of this work were conducted employing an epi-fluorescence microscope. To understand the description of the experimental set-up, let us briefly introduce the working principle of such a microscope.

The basis for fluorescence is the ability of certain substances to emit light after previously absorbing light. In particular, the substance, a so called fluorophore, absorbs a photon of wavelength $\lambda_{\text {excite }}$. Upon excitation, an electron within the fluorophore leaves its energetic ground state $S_{0}$ towards an energetically higher state, $S_{1}$, compare Fig. 4.7 A. Note that each energy state can have several vibra- 
tional levels, in the image denoted by 0 to 3 . In the depicted case, the electron enters the vibrational level 2. The electron now relaxes again to a energetically lower level. This happens in several steps. The initial relaxation goes to the lowest vibrational level within the energy state it is currently found in, so level 0 in $S_{1}$. This process is called vibrational relaxation and results in a reduction of energy but does not yield radiation. Next, the electron further decreases to the next lower energy level, $S_{0}$. This transition also goes along with a reduction of energy which is now detected in terms of a photon. As the difference in energy in the second step is smaller than the energy that originally caused the excitation, the emitted photon does exhibit a longer wavelength, hence $\lambda_{\text {excite }}<\lambda_{\text {emit }}$. This phenomenon is known as the Stoke's shift and the basis for all fluorescent imaging techniques. The ability of substances to fluoresce is employed for microscopy by specifically binding the molecules to structures one wants to study. The imaging is then conducted with e.g. a microscope set-up as depicted in Fig. 4.7 B. The light emitted by the light source is filtered by a so called excitation filter to reduce the wavelength spectrum of the light. Subsequently, the light is reflected by a dichroic mirror. A dichroic mirror has the ability to reflect light of a given wavelength band while others pass through it. The excitation light is then focused on the sample by an objective. When the fluorescent substance within the sample, often a fluorescent dye, absorbs the light, it will emit light in another wavelength according to the principle described above. The emitted light passes back through the objective as well as the dicroic mirror. It is thus of importance that a mirror is chosen whose transition between reflecting and transmitting is set between the wavelength' of the exciting and emitting light. The emitted light is then further filtered by an emission filter, similar to the previous excitation filter. The light is again focused by a lens within an ocular before being recorded with a camera. Note that this is the set-up of an epi-fluorescent microscope. More advanced methods and set-ups do exist but are not used for the described experiments.

For all experiments, an Olympus IX81 inverted research microscope was used in combination with a $60 \mathrm{X}, 1.35 \mathrm{NA}$ oil immersion objective and a Retiga 6000 CCD camera. For the fluorescence recordings, a FITC/Cy5 (Fluorescein isothiocyanate / Cyanine 5 dye) dual excitation and emission filters were used (compare Table 4.3). All fluorescent beads and dyes employed within this thesis were in either of theses two channels and their excitation and emission spectra are found in Fig 4.8. 
A

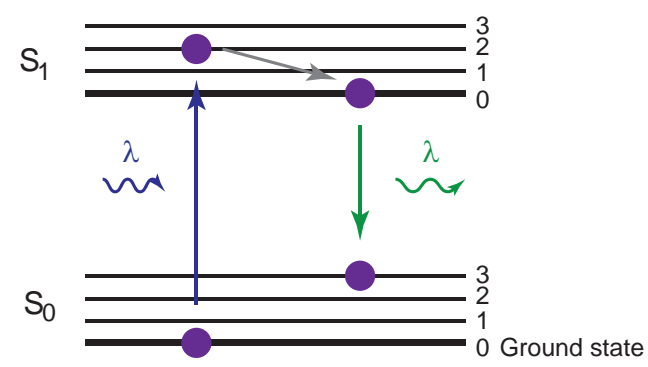

B

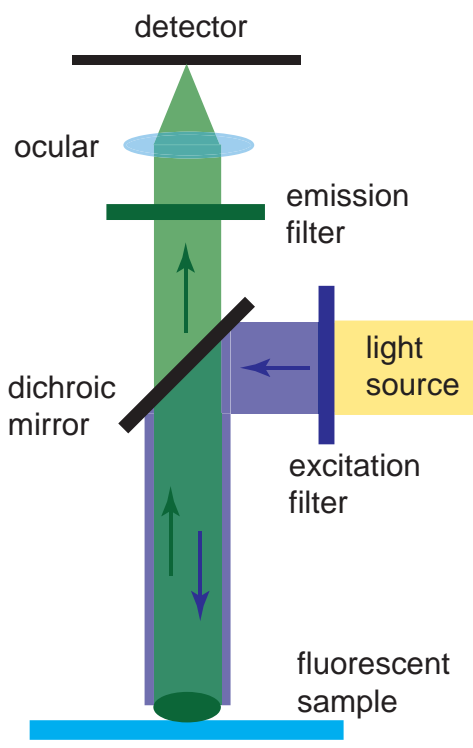

Figure 4.7.: A The principle of fluorescence. An electron (violet) in ground state is excited to a higher energy level $S_{1}$ and vibrational level (here 2) by absorbing light at a given wavelength (blue). The electron relaxes to the lowest vibrational level within the higher energy level (grey). This relaxation is not detected by emitted visible light. When the electron relaxes back to the lower energy level $S_{0}$, light is emitted at a longer wavelength (green) as the one that was previously absorbed. $\boldsymbol{B}$ The set-up of a fluorescence microscope. The light emitted from a light source (yellow) is filtered by an excitation filter (blue bar) to reduce the sprectrum of the light. The filtered light (here blue) is reflected at the dichroic mirror (black bar) to illuminate the fluorescent sample (green ellipse). The sample emits light in a different wavelength (green) according to the principle described in $\boldsymbol{A}$. The emitted light passes through the dichroic mirror and is further filtered by the emission filter (green bar). Following, the light is focused by a lens or ocular (blue ellipse) and recorded by a detector (black line). 


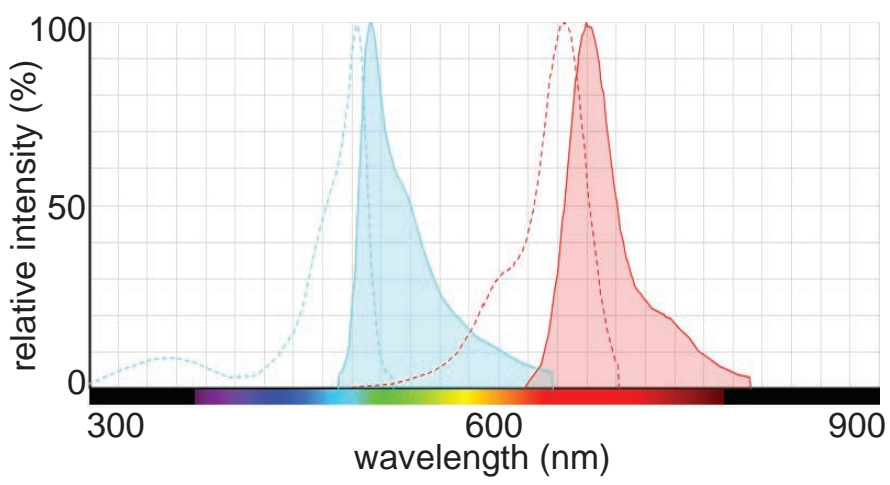

Figure 4.8.: The excitation (dashed) and emission (filled) spectra of the green fluorescent beads (blue) as well as the CellMask deep red and red fluorescent beads (red).

When imaging platelets, it was necessary to take into account that they are highly sensitive to light exposure. Too high light intensities or too long exposure times reduced first the amount of platelets spread followed closely by the decrease in the number of attached cells in the studied area. At the same time, an adequate amount of images per time needed to be taken to track the displacement of bead patters even on soft substrates as described in Section 5.2. Hence, an illumination set-up was chosen that allowed us to take a sufficient number of images per time while reducing the light exposure for the cells as much as possible. Depending on the conducted experiment, different illumination settings were taken which are summarised in the following subsections, separated according to the studied influencing factor.

\subsection{2. tTFM With Various Substrate Stiffnesses}

To ensure that all experiments were carried out as closely to physiological conditions as possible, an incubation chamber (INUG2EONICS, Table 4.3) was mounted onto the microscope stage and pre-heated to $37^{\circ} \mathrm{C}$ with a constant $\mathrm{CO}_{2}$ level of $5 \%$. In the chamber, the glass slide containing the gel was fastened using a customized sample holder.

The glass slide was taken out from the petri-dish and all fluid on both sides of the glass was removed carefully using a low-lint wipe. Comparing the dimensions of the substrate and the glass slide makes it obvious that not much glass was surrounding the gel. During imaging, this led to the problem that fluid left the substrate and flowed underneath the glass slide. As the objective was directly in contact with 
the sample, this resulted in platelet solution running onto the objective. To avoid this problem, after drying the glass, a hydrophobic pen (Mini Pap Pen, compare Table 4.3) was used to retrace the border of the substrate. Subsequently, the gel was gently washed with HT-BSA buffer using a pipette. The last round of buffer solution was kept on the substrate until usage to avoid drying out. The glass slide was now fastened in the chamber.

Using the FITC channel on the microscope, the lower and upper surface of the substrate was visualised by the two distinct bead layers found in these regions. The lower bead layer was characterised by a generally very high bead density with low background noise in the images. The upper layer normally showed a smaller amount of beads but overall high enough to be able to analyse the images as described in Section 5.2. The difference in the background signal stemmed from the fact that to image the upper bead layer, the light was focused through the gel, resulting in additional scattering within the sample. Noting the objective positions in height at the different bead layers, it was possible to approximate the thickness of the gel. Each gel was measured at least at five different positions on the substrate; one position in the middle and four positions distributed regularly over the border of the substrate. At the border positions, often multiple measurements were taken as the thickness gradient was extremely high for some substrates. The height range was noted for each gel. Overall, the average thickness of the substrates was found to be about $35 \mu \mathrm{m}$. For the recording, no positions was chosen at which the substrate was less than $20 \mu \mathrm{m}$ high to ensure that the platelets did react to the stiffness of the gel and not the glass underneath. Furthermore, at about $15 \mu \mathrm{m}$ thickness and below, the lower bead layer shone through to the upper layer, resulting in a very high background signal.

For the experiments, the remaining HT-BSA solution was carefully aspirated with a low-lint tissue without touching the gel itself. $291.6 \mu \mathrm{L}$ of the stained platelets were added as well as $32.4 \mu \mathrm{L}$ thrombin, resulting in a final concentration of $4 \mathrm{u} / \mathrm{mL}$ thrombin. The Cy5 channel of the microscope was switched on and an attaching cell was searched for. An attached cell appeared as a bright, round spot of about $3 \mu \mathrm{m}$ in diameter (compare Fig. 6.1 D). As soon as such a cell was discovered, the actual recording was started at this position.

For the recording, a compromise between image quality and influence of the energy input on the platelets was reached. As mentioned previously, platelets are lightsensitive and excessive light exposure was avoided by all means. At the same time, we needed a sufficiently high contrast between the beads and the background as well as a good time resolution. The lowest possible light exposure at which the 
beads were still distinguishable from the background by means of computational filter algorithms was a combination of $23 \%$ light intensity of the used xenon arc-lamp and an exposure of $50 \mathrm{~ms}$ each for both the beads and cells. As time resolution, a time step of $7.5 \mathrm{~s}$ was used. At shorter time steps, the cells were visibly affected compared to platelets situated at non-exposed positions. Longer time steps on the other hand resulted in too large deformations between images to still be analysable, especially when studying cells on softer gels. All recordings had a total length of $30 \mathrm{~min}$.

\subsection{3. tTFM in Combination With Microfluidics}

For the microfluidics experiments, a larger set-up was needed, including a syringe pump unit (syringe pump neMESYS, Table 4.3) in addition to the microscope. The cells and thrombin solutions were prepared as previously at the same concentrations of $2 \cdot 10^{7}$ cells $/ \mathrm{mL}$ and $40 \mathrm{u} / \mathrm{mL}$, respectively. Note that for these experiments, the platelets were not stained as the membrane dye was not stable enough for all preparation steps and the following recording. Additionally, as previously, some millilitres of HT-BSA buffer was aliquoted for washing the gels.

To start, the measuring chamber and the mixing channel was connected. Here, three pieces of tubing were cut, each about $20 \mathrm{~cm}$ of length, and inserted in the three inlets of the mixing channel. In the outlet of the mixing channel, one of the already connected tubes of the measuring chamber was inserted. To be able to fasten the finished chamber device into the incubation chamber while the mixing device was mounted onto the microscopy stage, the connecting tube was cut to a length of about $12 \mathrm{~cm}$. Hence, one tubing connected to the measuring device was cut to this length and then inserted into the outlet of the mixing channel. All newly inserted tubings were then glued with two-component glue.

As previously, the substrate was washed with HT-BSA buffer. Here, the entire device was flushed as done when assembling the measuring chamber device. The buffer solution was filled into a disposable $1 \mathrm{~mL}$ syringe (disposable syringe, Terumo Corporation, Table 4.3) without any air bubbles enclosed and mounted on a syringe driving unit. The outlet of the measuring chamber was opened and connected to the syringe so the entire device including all three inlets was flushed with the buffer. For the filling of the system, a flow rate of $800 \mu \mathrm{L} / \mathrm{h}$ was used. When the whole system was filled with buffer, all inlets and the outlet were again closed by melting the tubing. For this step, it was of utmost importance to avoid 
any kind of air pockets both in the tubing and the devices themselves as they remained during recording and disturbed or even blocked the fluid flow. A photo of the final device can be seen in Fig. $4.9 \mathrm{~A}$.

The platelets and thrombin were now filled into their respective syringes. Here, Hamilton gas tight glass syringes (Table 4.3) were used together with a cannula of $0.4 \mathrm{~mm}$ inner diameter (disposable hypodermic needle Sterican, Table 4.3). For the thrombin, two syringes each with a total volume of $250 \mu \mathrm{L}$ where filled and all air bubbles removed. To avoid remaining air in the needles, the needles were then exchanged and re-filled with liquid. For the platelets, a syringe with a total volume of $2.5 \mathrm{~mL}$ was used. As the platelet-HT-BSA solution tended to produce large air bubbles when pulling the liquid into the syringe with the small cannula used for the other syringes, a larger needle was used with a diameter of $0.9 \mathrm{~mm}$. This had the additional advantage of exerting less stress on the cells during filling. After the air from the syringe was removed, the cannula was exchanged to the smaller needle of $0.4 \mathrm{~mm}$ diameter. The syringes were now mounted onto the syringe pump on the fixed stage. The syringe pump was connected to the computer to be able to operate the flow rate dispensed from the syringes.

The measuring chamber was mounted into the incubation chamber using the customised sample holder. The mixing device was fastened to the microscopy stage using adhesive tape such that the outlet was pointing towards the incubation chamber without stretching the tubing. The inlets were pointed towards the syringes to keep the connecting tubes as short as possible. The outlet of the whole system was guided out of the incubation chamber into a reaction tube also fastened onto the stage with adhesive tape. This functioned as the waste collector. An image of the final set-up can be found in Fig. 4.9 B.

As with the static experiments, the substrate thickness was measured by focusing first on the lower bead layer followed by the upper bead layer and the height was calculated by the difference in objective position. To get an overview of the thickness of the whole gel, three sections were measured at multiple positions: the section near the inlet, a section at the central part and finally the section nearest to the outlet. Contrary to the static experiments, a ROI for recording was chosen before the attachment of any cell as the time-point of attachment varied greatly between experiments. Instead, a position was chosen that exhibited a thickness of at least $20 \mu \mathrm{m}$, a high bead density and was as little tilted as possible. Recordings were made in the middle along the width of the channel. The average substrate height was found to be $30 \mu \mathrm{m}$. 

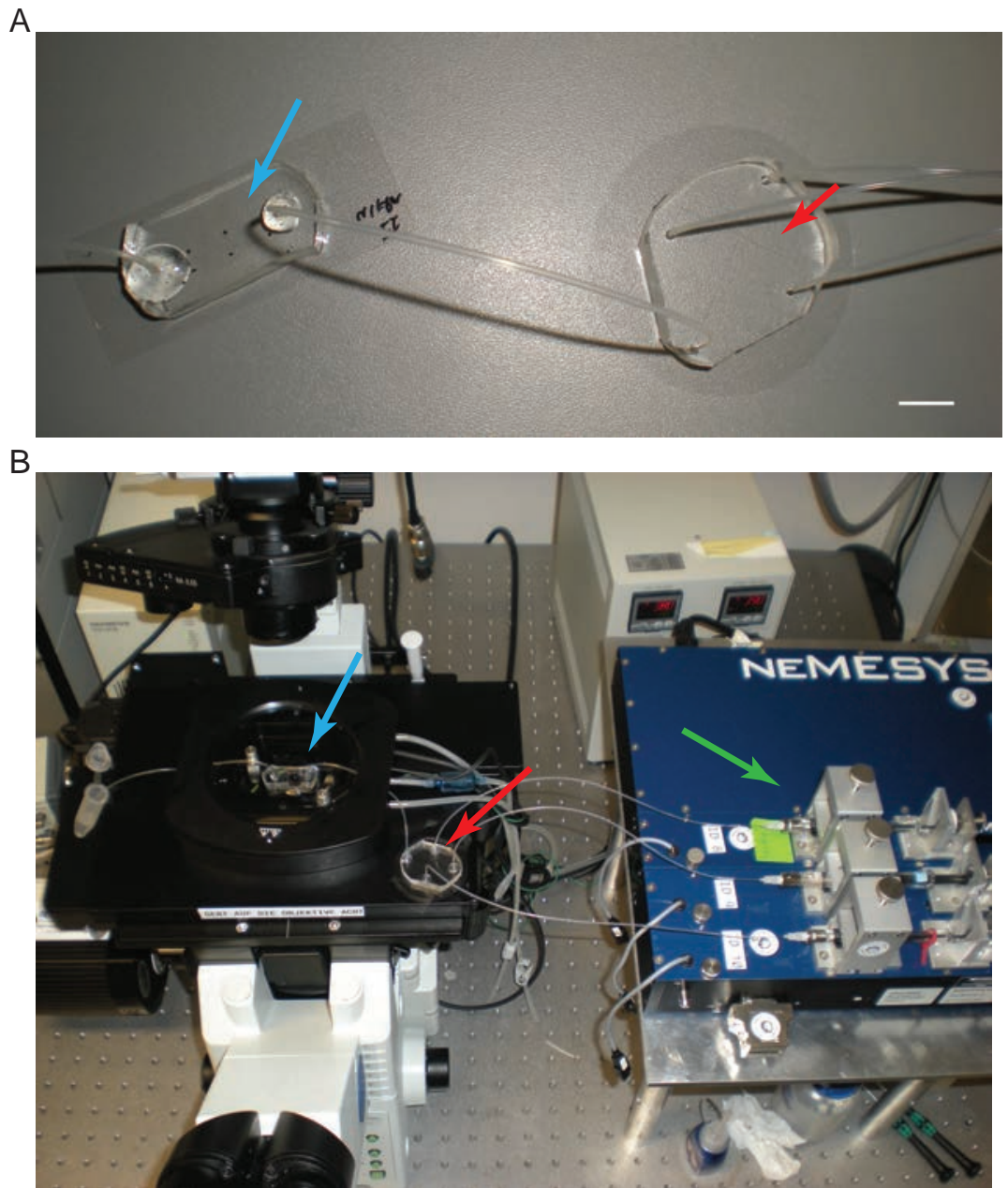

Figure 4.9.: $\boldsymbol{A}$ The assembled microfluidic device. The left device (blue arrow) shows the measuring chamber with an inlet (right) and an outlet (left). Here, the tubings are already sealed with glue to avoid leakage. On the right, the mixing channel is connected to the chamber (red arrow). It has one outlet (left, connected to the measuring chamber) and three inlets (right). During filling of the system with HT-buffer, the fluid is flushed from left to right, during recording from right to left. Scale bar: $1 \mathrm{~cm}$. $\boldsymbol{B}$ The entire set-up at the microscope. From right to left: The pumps (green arrow) supply the platelet solution (middle syringe) while the smaller outer syringes are filled with thrombin. The blending occurs within the mixing channel (red arrow) which is glued to the microscopy stage. From the channel, the fluid is transported into the measuring chamber located in the incubation chamber (blue arrow). On the outer left, the waste fluid leaves the chamber into the waste tube. 
The syringes were started at an arbitrary flow rate to initially expel remaining air from the cannula. When a droplet of the fluid developed at the needle, the end of the corresponding tubing was cut open and connected to the syringe. Note that no air was allowed in the tubing when connecting to the syringes as this remained in the system during recording. The whole system was now filled with both substances until all had entered the mixing device. It was of importance that both thrombin streams had reached the mixing region of the mixing device as they continued later at a comparably low speed during recording. Simultaneously, the platelet flow rate for the filling was not set too high to avoid unnecessary stress to the cells.

For the recording, the final flow rates of $300 \mu \mathrm{L} / \mathrm{h}, 500 \mu \mathrm{L} / \mathrm{h}$ and $700 \mu \mathrm{L} / \mathrm{h}$ were used, corresponding to an average velocity in the measuring chamber of $236 \mu \mathrm{m} / \mathrm{s}$, $393 \mu \mathrm{m} / \mathrm{s}$ and $550 \mu \mathrm{m} / \mathrm{s}$, respectively. The final concentration of the plateletthrombin mixture was set to be equal to that of the static experiments. Hence, after the initial filling of the mixing channel, the flow rates for the thrombin vs platelet syringes were set to a ratio of $1: 18$, i.e. for the $300 \mu \mathrm{L} / \mathrm{h}$ flow rate, the thrombin syringes delivered the solution at a flow rate of $15 \mu \mathrm{L} / \mathrm{h}$ each and the platelet syringe at $270 \mu \mathrm{L} / \mathrm{h}$.

The recording was done using the FITC channel with the settings as previously described. The cells were imaged using the BF channel. The bright field lamp was set to $3.2 \mathrm{~V}$ and the exposure time to $500 \mathrm{~ms}$. In total, the recording lasted for $1.5 \mathrm{~h}$.

Table 4.3.: Chemical products and tools used for all experiments and their suppliers.

\begin{tabular}{|l|l|}
\hline Chemical / Tool & Supplier \\
\hline $2 \%$ Bis-acrylamide & $\begin{array}{l}\text { Bio-Rad Laboratories Inc., Hercules, } \\
\text { CA, USA }\end{array}$ \\
\hline $40 \%$ Acrylamide & $\begin{array}{l}\text { Bio-Rad Laboratories Inc., Hercules, } \\
\text { CA, USA }\end{array}$ \\
\hline $\begin{array}{l}60 X \text { oil-immersion objective, UP- } \\
\text { lanSApo, NA 1.35 }\end{array}$ & Olympus, Hamburg, Germany \\
\hline APS & $\begin{array}{l}\text { Bio-Rad Laboratories Inc., Hercules, } \\
\text { CA, USA }\end{array}$ \\
\hline & \\
\hline
\end{tabular}




\begin{tabular}{|c|c|}
\hline \multicolumn{2}{|l|}{ continued from previous page } \\
\hline APTMS & Sigma-Aldrich, St. Louis, MO, USA \\
\hline BSA Macs BSA stock solution & $\begin{array}{l}\text { Milteny Biotech, Bergisch Gladbach, } \\
\text { Germany }\end{array}$ \\
\hline CellMask DeepRed, 649/66 nm & $\begin{array}{l}\text { Thermo Fisher Scientific Inc., } \\
\text { Waltham, MA, USA }\end{array}$ \\
\hline Centrifuge $5810 \mathrm{R}$ & Eppendorf AG, Hamburg, Germany \\
\hline $\begin{array}{l}\text { Disposable hypodermic needle Steri- } \\
\text { can, various sizes }\end{array}$ & $\begin{array}{l}\text { B.Braun Melsungen AG, Melsungen, } \\
\text { Germany }\end{array}$ \\
\hline Fibrinogen & $\begin{array}{l}\text { CalBiochem-Merck KGaA, Darm- } \\
\text { stadt, Germany }\end{array}$ \\
\hline $\begin{array}{l}\text { FITC/Cy5 dualband emission filter, } \\
537 \mathrm{~nm} \text { and } 694 \mathrm{~nm}\end{array}$ & $\begin{array}{l}\text { AHF Analysetechnik AG, Tübingen, } \\
\text { Germany }\end{array}$ \\
\hline $\begin{array}{l}\text { FITC/Cy5 dualband excitation fil- } \\
\text { ter, } 470 \mathrm{~nm} \text { and } 628 \mathrm{~nm}\end{array}$ & $\begin{array}{l}\text { AHF Analysetechnik AG, Tübingen, } \\
\text { Germany }\end{array}$ \\
\hline $\begin{array}{l}\text { FluoSpheres,carboxylate-modified } \\
\text { microspheres, } 40 \mathrm{~nm} \text { diameter, } \\
\text { yellow-green, } 505 / 515 \mathrm{~nm}\end{array}$ & $\begin{array}{l}\text { Thermo Fisher Scientific Inc., } \\
\text { Waltham, MA, USA }\end{array}$ \\
\hline $\begin{array}{l}\text { FluoSpheres,carboxylate-modified } \\
\text { microspheres, various sizes, red, } \\
660 / 680 \mathrm{~nm}\end{array}$ & $\begin{array}{l}\text { Thermo Fisher Scientific Inc., } \\
\text { Waltham, MA, USA }\end{array}$ \\
\hline $\begin{array}{l}\text { Glass cover slips, thickness No } 1 \text {, var- } \\
\text { ious sizes }\end{array}$ & VWR, Radnor, PA, USA \\
\hline Glutaraldehyde & $\begin{array}{l}\text { Polysciences Inc., Warrington, PA, } \\
\text { USA }\end{array}$ \\
\hline $\begin{array}{l}\text { Incubation chamber } \quad \text { INUG2E- } \\
\text { ONICS }\end{array}$ & Tokai Hit Ltd. Co., Shizuoka, Japan \\
\hline Incubator HeraCell 150 & $\begin{array}{l}\text { Thermo Fisher Scientific Inc., } \\
\text { Waltham, MA, USA }\end{array}$ \\
\hline IX81 inverted microscope & Olympus, Hamburg, Germany \\
\hline $\begin{array}{l}\text { KimTech Science, low-lint precision } \\
\text { wipes }\end{array}$ & $\begin{array}{l}\text { Kimberly-Clark Corporation, Irving, } \\
\text { TX, USA }\end{array}$ \\
\hline Microscopy slides, cut edges & VWR, Radnor, PA, USA \\
\hline Mini Pap Pen & $\begin{array}{l}\text { Thermo Fisher Scientific } \\
\text { Waltham, MA, USA }\end{array}$ \\
\hline
\end{tabular}




\begin{tabular}{|c|c|}
\hline \multicolumn{2}{|l|}{ continued from previous page } \\
\hline MT-ARC/Xe lamp & Olympus, Hamburg, Germany \\
\hline Oven DryLine & VWR, Radnor, PA, USA \\
\hline Plasma Cleaner PDC-32G & Harrick Plasma, Ithaca, NY, USA \\
\hline Plus One Repel Silane & GE Healthcare, Little Chalfont, UK \\
\hline $\begin{array}{l}\text { Polyethylene tubing, } 0.38 \mathrm{~mm} \text { inner } \\
\text { diameter }\end{array}$ & $\begin{array}{l}\text { Becton, Dickinson and Company, } \\
\text { Franklin Lakes, NJ, USA }\end{array}$ \\
\hline Prostaglandin $\mathrm{E}_{1}$ & $\begin{array}{l}\text { Cayman Chemical Company, Ann } \\
\text { Harbor, MI, USA }\end{array}$ \\
\hline Puncher, $0.75 \mathrm{~mm}$ & $\begin{array}{l}\text { World Precision Instruments, Sara- } \\
\text { sota, FL, USA }\end{array}$ \\
\hline QImaging Retiga 6000 CCD camera & QImaging, Surry, BC, Canaday \\
\hline Reaction tubes, various sizes & VWR, Radnor, PA, USA \\
\hline Rotator, MACSmix Tube Rotator & $\begin{array}{l}\text { Miltenyi Biotech GmbH, Bergisch } \\
\text { Gladbach, Germany }\end{array}$ \\
\hline $\begin{array}{lll}\text { Scalpels, } & \text { Surgical } & \text { Disposable } \\
\text { Scalpels } & & \\
\end{array}$ & $\begin{array}{l}\text { B.Braun Melsungen AG, Melsungen, } \\
\text { Germany }\end{array}$ \\
\hline Sulfo-SANPAH & $\begin{array}{l}\text { Thermo Fisher Scientific Inc., } \\
\text { Waltham, MA, USA }\end{array}$ \\
\hline $\begin{array}{l}\text { Syringe, Hamilton gas-tight, Series } \\
1000\end{array}$ & Hamilton, Bonaduz, Switzerland \\
\hline Syringe, disposable, $1 \mathrm{~mL}$ & Terumo Corporation, Tokyo, Japan \\
\hline $\begin{array}{l}\text { Syringe, disposable, BD Discardit, } \\
2 \mathrm{~mL} \text { and } 5 \mathrm{~mL}\end{array}$ & $\begin{array}{l}\text { Becton, Dickinson and Company, } \\
\text { Franklin Lakes, NJ, USA }\end{array}$ \\
\hline $\begin{array}{l}\text { Syringe pump neMESYS, used for } \\
\text { Hamilton syringes }\end{array}$ & $\begin{array}{l}\text { neMESYS, Centoni GmbH, Kor- } \\
\text { bußen, Germany }\end{array}$ \\
\hline $\begin{array}{l}\text { Syringe pump LA-30, used for dis- } \\
\text { posable syringes }\end{array}$ & $\begin{array}{l}\text { Landgraf Laborsysteme HLL GmbH, } \\
\text { Langenhagen, Germany }\end{array}$ \\
\hline TEMED & $\begin{array}{l}\text { Bio-Rad Laboratories Inc., Hercules, } \\
\text { CA, USA }\end{array}$ \\
\hline Thrombin from human plasma & Sigma-Aldrich, St. Louis, MO, USA \\
\hline $\begin{array}{l}\text { UHU Plus Sofortfest } 2-\mathrm{K} \text { - } \\
\text { Epoxidkleber Transparent }\end{array}$ & $\begin{array}{l}\text { OBI Group Holding SE and Co. } \\
\text { KGaA, Wermelskirchen, Germany }\end{array}$ \\
\hline
\end{tabular}





\section{Development of an Analysis Algorithm}

In this chapter, the theoretical background for the data analysis as well as the interpretation is presented. Let us briefly recap the major point we derived in the previous chapter. The key mathematical tool is the relation between the forces exerted on an elastic medium and its resulting displacements. Under the experimental conditions used in the presented investigations, the Boussinesq solution to the equation of equilibrium of an elastic medium bounded by a plane provides a useful model (Ref. [59], §1.8, and Ref. [106], Section II.1.2):

$$
\int_{\mathbb{R}^{2}} \mathbf{G}\left(\mathbf{x}^{\prime}-\mathbf{x}\right) f\left(\mathbf{x}^{\prime}\right) d \mathbf{x}^{\prime}=\mathbf{u}(\mathbf{x}) .
$$

This equation connects the surface forces $f$ and the surface displacements $\mathbf{u}$ on an infinite elastic material. Letting $\mathbf{x}=(x, y)$, the Green's function $\mathbf{G}$ is given by

$$
\mathbf{G}(\mathbf{x})=\frac{1+\nu}{\pi E r^{3}}\left(\begin{array}{cc}
(1-\nu) r^{2}+\nu x^{2} & \nu x y \\
\nu x y & (1-\nu) r^{2}+\nu y^{2}
\end{array}\right)
$$

where $r=\sqrt{x^{2}+y^{2}}, E$ is the Young's modulus and $\nu$ is the Poisson's ratio. We develop an algorithm tailored to the special situation of measuring the forces of very small cells which exert exceedingly high forces. We intend to emphasize that the algorithm is rather general such that it can easily be adapted to similar situations.

The analysis algorithm to calculate the forces exerted of the platelets is divided into two parts: First, the PIV algorithm for determining the displacement field is described in Section 5.2, followed in Section 5.4 by a discussion of the regularisation procedure usually used during the FTTC algorithm for computing the force field. Here, we emphasis on the circumstances under which regularisation is needed or, in our case, not needed. Another method for determining the displacement field is the so called optical flow algorithm. It is described in detail in Section 5.3 and its properties are compared to the PIV-based algorithm. As part of the data interpretation, this is followed by the calculation the force dipole ratio of 
contracting cells derived in Section 5.5. Note that several parts of this chapter are part of Hanke et al. [36]. In particular, these include Sections 5.2, 5.3, 5.4.3, 5.4.4, 5.4 .5 and 5.5 .

The final algorithm becomes rather complex. For its construction and understanding it is useful to have a simplified example at hand. This allows us to demonstrate certain properties of the algorithmic components. Measured data could be used when one is interested in whether the algorithm is applicable to real data. However, when searching for possible errors or pitfalls, it is more feasible to use a data set where the answer is known. To this end, an artificial data set is constructed that shows behaviour similar to the recorded data of the contracting platelets but can be changed in its characteristics. It is described in Section 5.1.

\subsection{Construction of Artificial Auxiliary Data Set}

The test data set includes a number of images similar to the bead images recorded. Around a number of positions randomly sampled, a Gaussian intensity profile is assumed. Here, the intensity corresponds to different gray values within the image, mimicking the idealised images of the beads. Additionally, Gaussian noise is added to the images. Gaussian noise is a noise distribution associated with images recorded at, e.g. low intensities. An example of the constructed, noisy image can be found in Fig. 5.1, a recorded image in Fig. 6.2 A. From image to image, the beads are moved to emulate the contraction of a cell situated on the substrate.

The movement inside the images is constructed to be close to the actual expected bead movement. This behaviour is characterised both by the shape and velocity profiles of beads' movement. Generally, on flat substrates, platelets have been shown to adopt a near elliptical shape. [102]. Thus, it is reasonable to approximate the area of contraction under the cell by that of a circle. Now, let us consider a simple representation of a velocity field which similarly can be observed beneath the cell.

For several cell types, among them platelets [107], it has been shown that the contraction is highest along the periphery of the cell $[3,46]$. Thus, we can model the contraction in our auxiliary data set as being observed along the contour of the circle representing the cell. We further assume that the contraction is equal at each point. With respect to the time, we use the ansatz

$$
R(t)=R_{0} e^{-\beta t}
$$


for the radius of the contraction pattern where $\beta>0$ and $R_{0}$ is the initial radius. This is a simple model of a contracting circle with $R(t) \rightarrow 0$ for $t \rightarrow \infty$.

For the velocity described by the artificial beads, we assume them to be embedded in a gel, hence, it holds that

- the largest velocity is found on the cell periphery;

- in the cell centre, the velocity is zero;

- far away from the cell, the velocity is zero;

- the velocity is always in the direction of the contractions, $i$. e. going towards the cell centre.

We assume that the gel is homogeneous, hence, the velocity field is radially symmetric. Setting the origin in the cell centre, we can describe the velocity as $\mathbf{v}=\left(v_{r}, v_{\theta}\right)=\mathbf{v}(r)$ in spherical coordinates $(r, \theta)$. The ansatz for the velocity is then set to be

$$
v_{r}(r)=v_{0} r^{n} e^{\gamma r}, \quad v_{\theta}=0 .
$$

Here, the exponent $\gamma$ is chosen in such a way that the maximum in $\mathbf{v}$ is found at the current radius $R(t)$. It follows that

$$
\gamma=\gamma(t)=-\frac{n}{R(t)}
$$

The term $r^{n}$ accounts for the fact that the velocity is 0 at the center of the cell. By the exponent $n$ we can control the rate of decay against 0 . The displacement of the beads themselves is then given by

$$
\begin{aligned}
& r^{\prime}=v_{r}(r, t), \\
& \theta^{\prime}=0
\end{aligned}
$$

in spherical coordinates.

For one data set, five images are created in which the circular cell contracts towards its centre, see Fig. 5.1 for an example. The same images are then inverted in time and added to the image stack. This creates the image sequence $1 \rightarrow 5 \rightarrow 5 \rightarrow 1$. This sequence is then repeated to a total of 10 contraction cycles. Several versions of the data set are created with changing bead density, cell radius, contraction speed as well as the level of image noise in order to confirm the robustness of the algorithm. In all data sets, the exponent $n$ is taken to be 1 . 


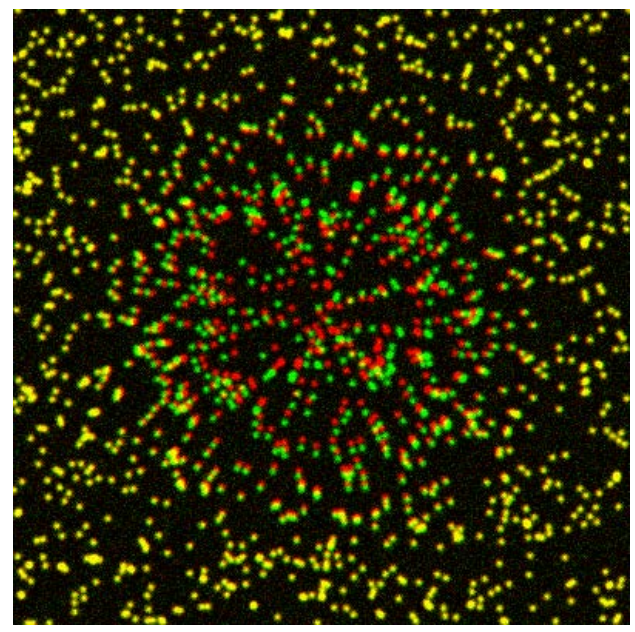

Figure 5.1.: Overlay of frame 1 and 5 of a contracting circle. Frame 1 denotes the relaxed state and is coloured in green, frame 5 denotes the maximal deformed state and is coloured in red. In the images, $10 \%$ Gaussian noise is included.

\subsection{PIV on Image Data of Small, Highly Contractile Cells}

We must solve Boussinesq's equation Eq. (5.1). To do so, we first need to determine the displacements of the beads before being able to calculate the forces. There exists a multitude of different algorithms to calculate the displacements from a given set of images as presented in Section 2.2.2. In the following, we employ the PIV algorithm [95] and adapt it for time-resolved TFM measurements.

\subsubsection{Time-Resolved PIV}

Let us first consider the general idea of PIV. The aim of PIV is to determine the deformation of the substrate between two images taken at time points $t_{i}$ and $t_{j}$, respectively. The deformation is made visible by a set of fluorescent beads which we assume to be fixed within the gel. Instead of direct bead-tracking as done in PTV, we now study the deformation of a gel patch, $i$. $e$. we track patterns of beads. The following steps are depicted in Fig. 5.2. 


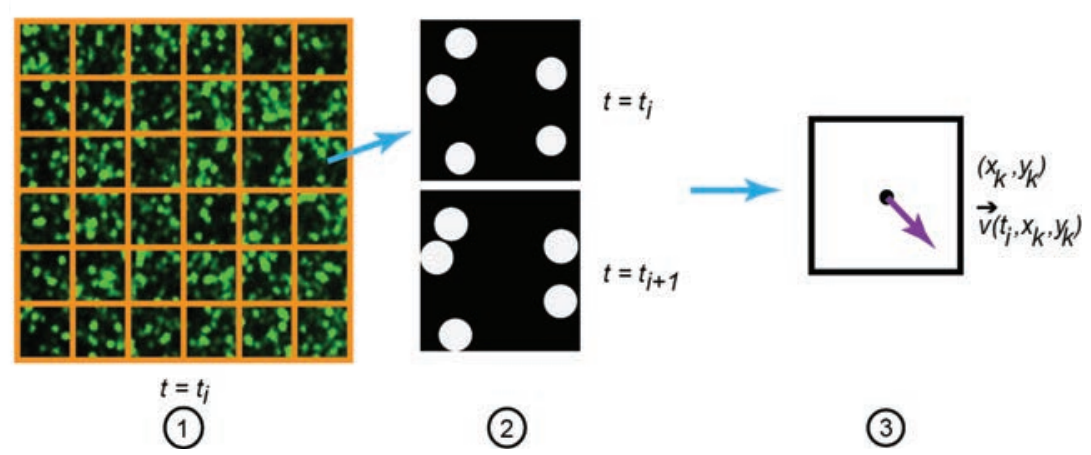

Figure 5.2.: A simplified representation of the PIV algorithm used. In step 1, the bead image (underlying image) is subdivided into smaller sub-windows of equal size and spacing (orange). Taking an arbitrary sub-window, assume the white circles in step 2 describe the position of the beads at $t=t_{i}$. At time point $t_{i+1}$, all beads have moved slightly differently from each other but with a preferred direction. By cross-correlation of the images in step 2 and considering the time difference, the statistical mean velocity (purple) can be determined for the entire sub-window (step 3). The image is taken from Hanke et al. [36].

To track the movement of a bead pattern or group of beads, we first sub-divide the first image at $t_{i}$ into sub-areas. This is done in a regular fashion such that each area, henceforth called sub-windows, is square, of equal size, equidistant and covers the entire image (compare step 1 in Fig. 5.2). The same sub-division is done with the image recorded at $t_{j}$.

Consider now two sub-windows at the same position within the different images (step 2). We assume that the sub-window is sufficiently small so only translation is seen but no rotation. Furthermore, the difference in displacement of the single beads between the time points are approximately the same. Then the displacement of the window is given by the cross-correlation where the peak in the correlation matrix corresponds to the most probable displacement. Note that this is equivalent to the statistical mean displacement of the window. This analysis is now performed for each sub-window pair in the images. It is important to keep in mind that deformations that result in beads leaving the windows or that changes the pattern considerably, lead to a failure to determine the peak in the cross-correlation matrix. This phenomenon is called loss-of-pairs.

In this work, we do not only consider two time points at which the bead positions are recorded but we plan to study a whole sequence of images taken over a longer time interval. While we still always compare two images with each other, this comparison must be performed with more image pairs. Especially, we intend to 
track the bead movement starting from an initial, relaxed position, which we set to be the time point of cell attachment. We have now two options: Either, we compare each image at time point $t_{i}$ to the initial, relaxed state at time $t_{0}$, or we always compare two subsequent images at times $t_{i}$ and $t_{i+1}$ with each other. The former option has been used previously in other studies [3,20,46]. However, this approach has one major drawback in that is cannot account for larger deformations that may occur for longer recording times or for highly contractile cells.

To avoid the loss of information for larger deformations, the second approach is more suitable. Here, one only has to consider a sufficiently small time interval $\Delta t$ between images but the total length of recording time becomes un-important. However, the question now arises how to determine the total deformation at a given time point $t_{i}$ compared to $t_{0}$ if we only calculate the instantaneous displacement. Summing up the displacements within the static sub-windows does not yield the desired result as becomes clear if one considers a spatially highly fluctuating displacement field. In other words, we do not need the displacement $\mathbf{u}(\mathbf{x}, t)$ but the displacement $\mathbf{u}(\mathbf{x}(t), t)$.

As an alternative to summing up static sub-windows, another ides is to move the sub-windows forward over time with the current displacement. However, this means that over time, if we assume a monotonously contractile behaviour, areas containing no sub-windows appear. This leads to larger areas with no displacement information being available.

We choose a third approach in this work. Here, we keep the grid of sub-windows static for all frames. In contrast to the previous approach, we introduce the concept of Lagrangian markers that are moved over time, starting at a known position at $t_{0}$. Effectively, this means that we decouple the determination of the local displacement field (done by using the physical beads) from the calculation of the global displacement fields where artificial Lagrangian markers are utilised.

After comparing two images taken at $t_{i}$ and $t_{i+1}$ and considering $\Delta t$, the PIV algorithm provides a velocity field $\mathbf{v}_{i}=\mathbf{v}_{i}(\mathbf{x})$ (compare step 3). For the tracking of Lagrangian markers later it is convenient to have the velocity field defined for all instances of time $t$. The easiest method to do this is

$$
\mathbf{v}(\mathbf{x}, t)=\mathbf{v}_{i}(\mathbf{x}), \quad t_{i} \leq t<t_{i+1}
$$




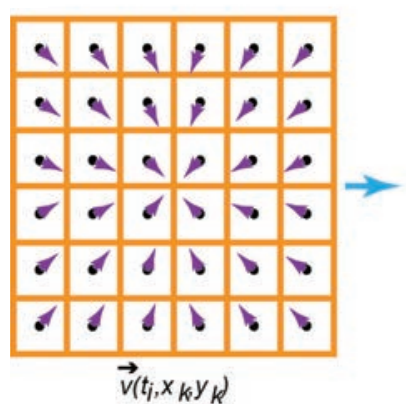

(1)

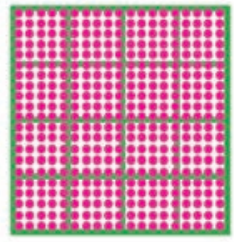

(2)

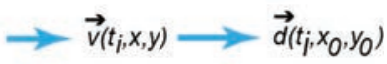

(3)

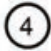

Figure 5.3.: The Lagrangian marker tracking algorithm. All velocity vectors (purple) are assigned to the mid-point of their corresponding sub-window (black dots, step 1). At time point $t_{0}$, Lagrangian markers are distributed over the image considered (pink dots, step 2). Note that the outer sub-windows are disregarded in this analysis, hence, the image is smaller in size (dark green grid). By interpolation, the velocity field is calculated at any given position (step 3) and, by tracking the movement of the markers, the displacement relative to the starting point can be calculated (step 4). The image is taken from Ref. [36].

\subsubsection{Recalculating the Displacement Fields - Tracking of Lagrangian Markers}

To calculate the displacement of the beads in our substrate over time, let us consider a concept used in fluid mechanics of changing the frame of reference when studying a fluid flow [97]. In fluid mechanics, we can observe the flow of substance within an area of interest either from an Eulerian or a Lagrangian frame of reference. In the Eulerian specification of a flow field, we chose a static point of reference and study how the flow changes in this point. Specifically, this observation method is the foundation of the PIV algorithm described above where we study the changes inside a rigid sub-window between images. Contrary, in the Lagrangian specification of the flow field, we study particles inside the flow and observe their trajectory over time. These particles may be real physical particles. This is the idea behind the embedding of beads inside deformable polymers as done in TFM and, from a computational point of view, the direct tracking using PTV. However, the Lagrangian approach can also be used in a completely computational procedure as is done here. In the following, by introducing the concept of Lagrangian markers, we transform the Eulerian PIV algorithm into a Lagrangian approach. The description of the algorithm can be followed step by step in Fig. 5.3. 
After the PIV calculation, we have a set of instantaneous velocity fields discrete in both time and space. For each sub-window, we know the statistical mean velocity for the entire area. Assuming that we interpolate the velocity field with respect to space, the setting of Eq. (5.8) provides then a velocity field $\mathbf{v}(\mathbf{x}, t)$ defined for all points $\mathbf{x}$ in space and instances $t \geq t_{0}$ in time.

At this stage, consider the Lagrangian approach of studying a flow field. Were we to have some kind of particles in the continuous velocity field, we could observe their motion over time from one frame to another. A first idea is to use the physical beads even as markers. However, this is not an option due to their tendency of building clusters, making it difficult to track them. Instead, we utilise artificial particles. These particles are a completely synthetic set of positions across our frame without any physical properties such as mass or dimension. To distinguish them from the physical beads in our substrate, these particles are hence called Lagrangian markers.

Let $\overline{\mathbf{x}}(t)$ denote the position of a Lagrangian marker at time $t$. This marker has the position $\overline{\mathbf{x}}_{0}=\overline{\mathbf{x}}\left(t_{0}\right)$ at the start $t_{0}$ of the recording. Hence, its displacement $\overline{\mathbf{u}}(t)$ at time $t$ is given by

$$
\overline{\mathbf{u}}(t)=\overline{\mathbf{x}}(t)-\overline{\mathbf{x}}\left(t_{0}\right) .
$$

In order to determine the displacement field, all we have to do is, therefore, to trace a sufficiently large set of markers. This is accomplished by solving the ordinary differential equation

$$
\frac{d}{d t} \overline{\mathbf{x}}(t)=\mathbf{v}(\overline{\mathbf{x}}(t), t)
$$

subject to the initial condition $\overline{\mathbf{x}}\left(t_{0}\right)=\overline{\mathbf{x}}_{0}$ for many arbitrary initial positions $\bar{x}_{0}$. Thus, it is essential to know the velocity at a given, arbitrary position at any time point. Here, we interpolate in such a way that the velocity value of each sub-window is assigned to the sub-window's center (step 1).

A finite set of Lagrangian markers is now distributed on the first frame at time $t_{0}$ such that the domain is densely packed (step 2). They are each defined by their starting position $\overline{\mathbf{x}}\left(t_{0}\right)$. For each position, we now determine the velocity according to our assumption made in Eq. (5.8) (step 3) and move the marker to the next frame. This procedure is repeated for all markers and all frames until the end. Giving their initial position, we can now re-calculate the total displacement according to Eq. (5.9) (step 4).

To ensure that no marker leaves the image frame that we study, additional Dirichlet boundary conditions are included at this point. As a consequence, we assume that the velocity at the boundary is zero everywhere and all markers reaching the 
boundary get an assigned velocity of zero, a so called "sticky edge condition". This assumption is justified by the fact that all studied cells are cut from the larger recording in such a way that no deformations are detected at the image edges.

Sections 5.2.1 and 5.2.2 describe the mathematical concept of the calculation of the displacement over time in an ROI. In the algorithm, several statistical and numerical methods and approximations are employed. This introduces errors and uncertainties to the calculations that cannot be avoided but reduced in their magnitude. In the following, these improvements are described.

\subsubsection{Reduction of Errors - Double Comparisons and Symplectic Methods}

Let us first consider the PIV algorithm. We determine the velocity vectors via cross-correlation which is a statistical method. We calculate here the statistical most probable mean velocity of our small sub-window. While we include tests to ensure that the main peak in the correlation space is really a certain, significantly higher peak than other peaks, the result still includes errors. These errors become apparent if we take a closer look at our contractile circle described above. In total, we only use five images which we first stack in one order followed by the inverse order. Theoretically, we should thus re-gain a displacement of 0 after 10 images. However, this is generally not the case. Instead, we note a slight off-set in the displacement due to small uncertainties in the PIV. In other words, the algorithm does not yield the same velocity magnitude at every point in space if a pair of images is first compared in one direction in time followed by the inverse order. An example can be see in Fig. 5.4, panels $\mathbf{A}$ to $\mathbf{C}$.

To reduce the errors at this step, all images are always be compared in both directions, once forward and once backwards in time. The final velocity field is then given by the mean of both results where consideration is given to the direction of the corresponding vector fields. As an additional advantage, subwindows where no velocity can be determined in one direction may yield a result when considering the reverse order, thus increasing the robustness of the method. Sub-images which are still missing any velocity vector are approximated by local Kriging interpolation [70]. Additionally, the sub-windows are in a following step reduced in size by halving the edge length. The same analysis as described before is done, taking the previously determined velocity vectors as guide lines for the new grid size. 


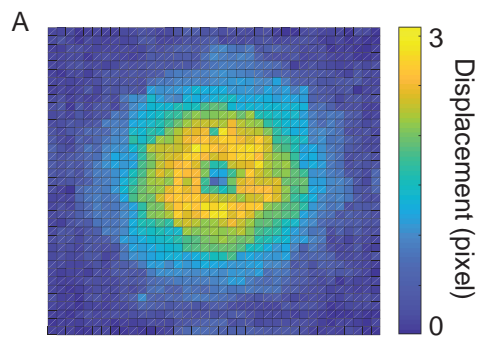

B
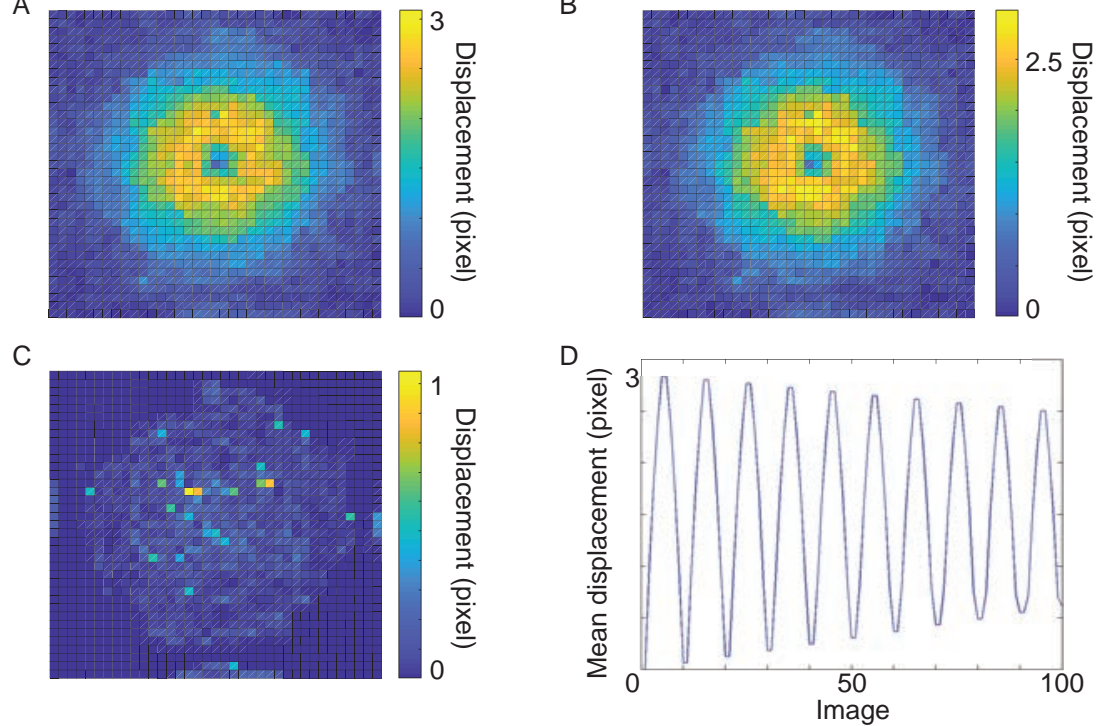

Figure 5.4.: Comparison of the first two frames of the contractile circle. A shows the comparison forward in time and $\boldsymbol{B}$ backwards in time. The displacement is given in pixels. The difference in displacement is displayed in C. As can be seen, the difference is not zero as would be expected from theory. $\boldsymbol{D}$ Using an Euler forward interpolation during marker tracking on ten consecutive contraction circles yields a positive off-set for the relaxed state. Additionally, a smaller decline can be seen at the point of maximum contraction. 
Next, consider the tracking of the Lagrangian markers. To solve Eq. (5.10), a numerical method has to be chosen. The simplest and fastest way is an forward Euler approach, which means we rewrite the equation as

$$
\overline{\mathbf{x}}\left(t_{i+1}\right) \approx \overline{\mathbf{x}}\left(t_{i}\right)+\Delta t \cdot \mathbf{v}\left(\overline{\mathbf{x}}\left(t_{i}\right), t_{i}\right)
$$

for a given time point $t_{i}$ and the corresponding velocity field. However, this yields an increasing positive off-set in the displacement. This approach adds onto the displacement as if we were adding energy to our system. The added displacement is noticeable when calculating the displacement at successive relaxed states in our example as shown in Fig. 5.4 D. It would be advantageous to use energypreserving methods, leading us to consider symplectic methods. One such method is the implicit mid-point method. The implicit mid-point method approximates Eq. (5.10) numerically by

$$
\overline{\mathbf{x}}\left(t_{i+1}\right) \approx \overline{\mathbf{x}}\left(t_{i}\right)+\Delta t \cdot \mathbf{v}\left(\frac{\overline{\mathbf{x}}\left(t_{i}\right)+\overline{\mathbf{x}}\left(t_{i+1}\right)}{2}, t_{i}+\frac{\Delta t}{2}\right)
$$

Besides being symplectical it has the advantage of being a second order method with respect to time thus potentially more accurate than the Euler methods.

The velocity field at time point $t_{i}+\frac{\Delta t}{2}$ is simply given by Eq. (5.8). But which location should we consider? Where is $\left(\overline{\mathbf{x}}\left(t_{i}\right)+\overline{\mathbf{x}}\left(t_{i+1}\right)\right) / 2$ ? To determine the appropriate location, we employ an iterative method, for example the fixed-point iteration. The fixed-point iteration is given by

$$
\overline{\mathbf{x}}^{m+1}=\mathbf{F}\left(\overline{\mathbf{x}}^{m}\right), \quad m=0,1, \cdots
$$

where the current approximation $\overline{\mathbf{x}}^{m+1}$ of the real solution $\overline{\mathbf{x}}\left(t_{i+1}\right)$ is given by inserting the previous approximation $\overline{\mathbf{x}}^{m}$ into the function $\mathbf{F}(\bar{x})$. Our function $\mathbf{F}$ is here given by the right hand side of Eq. (5.12) where $\overline{\mathbf{x}}\left(t_{i+1}\right)$ is replaced by $\overline{\mathbf{x}}$,

$$
\mathbf{F}(\overline{\mathbf{x}})=\overline{\mathbf{x}}\left(t_{i}\right)+\Delta t \cdot \mathbf{v}\left(\frac{\overline{\mathbf{x}}\left(t_{i}\right)+\overline{\mathbf{x}}}{2}, t_{i}+\frac{\Delta t}{2}\right)
$$

We need an initial guess $\mathbf{x}^{0}$ for $\overline{\mathbf{x}}\left(t_{i+1}\right)$ which we gain by using the forward Euler method as denoted in Eq. (5.11)

$$
\overline{\mathbf{x}}^{0}=\overline{\mathbf{x}}\left(t_{i}\right)+\Delta t \cdot \mathbf{v}\left(\overline{\mathbf{x}}\left(t_{i}\right), t_{i}\right)
$$


Subsequently, Eq. (5.13) is solved iteratively until the difference between successive approximations of $\overline{\mathbf{x}}\left(t_{i+1}\right)$ is much smaller than the iterates $\overline{\mathbf{x}}^{m}$ themselves. This provides the result for the new position of the studied Lagrangian marker. In practice, it turns out that the number of iteration steps is usually not larger than $2-4$. Given our set of displacements over time, we are now able to calculate the traction forces using FTTC.

\subsection{Optical Flow}

To later compare the results gained with the above described algorithm to another existing approach, an alternative analysis algorithm is used. We applied the aforementioned optical flow analysis. Contrary to the PIV approach, the optical flow does not track patterns formed by beads but instead so called 'features'. In our case, this is equivalent with the beads or even smaller bead clusters. These features or beads must first be found within an image. Considering the illuminated image recorded of a bead, one expects a near Gaussian intensity profile for each of the beads. This change in intensity can be used to detect the needed features by the so called Shi-Tomasi corner tracking algorithm [109,119]. The following algorithm was developed jointly with, and implemented by, D. Probst, University of Heidelberg.

In the Shi-Tomasi corner tracking, for each pixel position $(x, y)$, the nearest neighbourhood is considered. The neighbourhood is defined as a rectangular window of size $a \times b$ around the given pixel. For simplicity, let us here assume that $a=b$ thus defining a square region of interest. We are now searching for larger intensity changes in our image. Hence, consider what would happen if we were to change the position of our window around pixel $(x, y)$ by the translation vector $(\Delta x, \Delta y)$. The change in intensity can then be quantified as

$$
S S D(x, y)=\sum_{(u, v)}(I(u+\Delta x, v+\Delta y)-I(u, v))^{2},
$$

where $S S D$ denotes the sum of squared differences, $I$ the intensity of a given pixel and the sum is taken over all pixels $(u, v)$ within our window $a \times a$. Applying a Taylor expansion on the expression of the shifted intensity, we rewrite the previous equation as

$$
S S D(x, y) \approx \sum_{(u, v)}\left(\frac{\partial I(u, v)}{\partial u} \Delta x+\frac{\partial I(u, v)}{\partial v} \Delta y\right)^{2}
$$


Next, we define the structure tensor $\mathbf{S}(x, y)$ by

$$
\mathbf{S}(x, y)=\sum_{(u, v)}\left(\begin{array}{cc}
\left(\frac{\partial I(u, v)}{\partial u}\right)^{2} & \frac{\partial I(u, v)}{\partial u} \cdot \frac{\partial I(u, v)}{\partial v} \\
\frac{\partial I(u, v)}{\partial u} \cdot \frac{\partial I(u, v)}{\partial v} & \left(\frac{\partial I(u, v)}{\partial v}\right)^{2}
\end{array}\right)
$$

Note that $\mathbf{S}(x, y)$ is symmetric and positive semidefinite. Then, the problem of the intensity changes in our window during translation is approximated by

$$
S S D(x, y) \approx(\Delta x \Delta y) \mathbf{S}(x, y)\left(\begin{array}{c}
\Delta x \\
\Delta y
\end{array}\right)
$$

It follows from our definition that large changes in intensity mean a localised bead where the degree of change is given by the eigenvalues of the structure tensor $\mathbf{S}(x, y)$. Let now $R(x, y)=\min \left(\lambda_{1}(x, y), \lambda_{2}(x, y)\right)$ be the smallest eigenvalue of $\mathbf{S}(x, y)$. Then we define a threshold for the intensity change $q \cdot R_{\max }$ such that $R_{\text {max }}=\max _{(x, y)} R(x, y)$ and $q$ is a quality factor. Thus all pixels with $R(x, y)$ larger than the threshold are counted as a bead. The quality factor is used to reduce the influence of image noise. As this approach may still lead to errors due to larger bead clusters, detected features also need to show a minimum defined distance towards each other.

We have now found a set of detectable features as well as their starting position at $t_{0}$. The displacement of each feature is then tracked using the optical flow algorithm. Specifically, here, we use the pyramidal Kanade-Lucas-Tomasi (KLT) algorithm, see Refs. [8,71]. For the reasons stated in Section 5.2.1, we again replace the commonly used strategy of image comparison at time-points $t_{0}$ and $t_{i}$ by the comparison of successive image. Let us denote the time difference between images $t_{i}$ and $t_{i+1}$ as $\Delta t$. For small intensity changes over time, we can approximate

$$
I(x, y, t) \approx I(x+\Delta x, y+\Delta y, t+\Delta t)
$$

if the studied bead moves by the vector $(\Delta x, \Delta y)$. If we assume that $\Delta t$ becomes infinitesimal small and approaches 0 , this equation is well approximated by the optical flow equation

$$
\frac{\partial I(x, y)}{\partial x} v_{x}+\frac{\partial I(x, y)}{\partial y} v_{y}+\frac{\partial I(x, y)}{\partial t}=0
$$


As defined previously, $\mathbf{v}=\left(v_{x}, v_{y}\right)$ is the velocity, here of our studied feature. Given the calculated velocity vector of a given bead between two consecutive images, the displacement change is then be approximated by $\mathbf{u}=\mathbf{v} \Delta t$, assuming the velocity is constant between images.

It should be noted that Eq. (5.19) is under-determined since it is a scalar equation for the two unknowns $v_{x}$ and $v_{y}$. We thus choose a similar approach as done with the PIV algorithm and define a square window around each bead (feature) of size $s \times s$ and assume that pixels within this sub-window move in parallel to each other. Eq. (5.19) is then solved for each pixel within a sub-window in a least-square sense. Additionally, similar as the above described PIV algorithm, the edge length is halved after one pass of velocity calculation and the window area is reduced by a factor of 4 . This marks the pyramidal part of the algorithm, where again the displacement of the coarser grid is used as an off-set for the finer grid calculation. The halving of the edge length is repeated until a certain defined stop criterion is reached. Note that contrary to the PIV algorithm where the positioning of the grid of sub-windows stays constant, in the optical flow described here, the sub-windows move with the beads over time, resulting in an irregular grid.

Given the same reasons as stated in Section 5.2.3, to reduce statistical noise, we again change standard procedures of image comparison to compare images both forward and backwards in time and average the resulting displacement vectors. However, contrary to the aformentioned implicit mid-point method, for simplicity, an explicit Euler method is used.

In the PIV algorithm, when tracking the Lagrangian markers, we explicitly set the velocity of our markers to 0 at the boundary by applying a Dirichlet boundary condition. As we do not employ any such tracking here, to ensure a 0 velocity at the edge of our ROI, we directly set all displacements near the border to 0 . This is achieved by using a Tukey filter in two dimensions in real space [123] before continuing with the FTTC part of the analysis.

\subsection{Regularisation in FTTC}

\subsubsection{Fourier Transforms in the Force Calculation}

Given our continuous displacement field determined in Section 5.2 or alternatively Section 5.3, we now want to calculate the forces exerted on the gel. For this, we need to solve Eq. (5.1). As can be seen, this equation is a convolution. Mapping this into a two-dimensional periodic space, we can transform the equation into 
Fourier space. This idea was first introduced by Butler et al. [10]. We briefly summarise their results with its mathematical derivation to provide a better understanding of the following section on the regularisation during FTTC.

If the transformation from real space into Fourier space is given by the operator $\mathcal{F}$, then it holds that

$$
\begin{aligned}
\mathcal{F}(\mathbf{G} * \mathbf{f}) & =\mathcal{F}(\mathbf{u}), \\
\mathcal{F}(\mathbf{G}) \cdot \mathcal{F}(\mathbf{f}) & =\mathcal{F}(\mathbf{u}) .
\end{aligned}
$$

The Fourier transform $\hat{\mathbf{G}}$ of Green's function in Eq. (5.2) for a wave vector $\mathbf{k}=$ $\left(k_{1}, k_{2}\right)$ is given by

$$
\hat{\mathbf{G}}\left(k_{1}, k_{2}\right)=\frac{2(1+\nu)}{E \cdot k} \cdot\left(\begin{array}{cc}
(1-\nu) k^{2}+\nu k_{2}^{2} & -\nu k_{1} k_{2} \\
-\nu k_{1} k_{2} & (1-\nu) k^{2}+\nu k_{1}^{2}
\end{array}\right)
$$

where $\nu$ is Poisson's ratio, $E$ is the Young's modulus and $k$ denotes the norm of $\mathbf{k}, k=|\mathbf{k}|=\sqrt{k_{1}^{2}+k_{2}^{2}}$. We can see that for each given wave vector, the corresponding matrix is symmetric and real. Eq. (5.1) then translates to

$$
\hat{\mathbf{G}}\left(k_{1}, k_{2}\right) \cdot \hat{\mathbf{f}}\left(k_{1}, k_{2}\right)=\hat{\mathbf{u}}\left(k_{1}, k_{2}\right)
$$

Note that the values of $\left(k_{1}, k_{2}\right)$ all have the form $k_{i}=$ (scale factor) * integer, where the scale factor depends on the units used for measuring pixel distances (e.g. pixels units vs. SI units) while the integers are equal to or larger than 0 and thus form a regular grid. As an example, a square system of 4 nodes is given by

$$
\left(\begin{array}{cccc}
\hat{\mathbf{G}}(0,0) & 0 & 0 & 0 \\
0 & \hat{\mathbf{G}}(1,0) & 0 & 0 \\
0 & 0 & \hat{\mathbf{G}}(0,1) & 0 \\
0 & 0 & 0 & \hat{\mathbf{G}}(1,1)
\end{array}\right) \cdot\left(\begin{array}{c}
\hat{\mathbf{f}}(0,0) \\
\hat{\mathbf{f}}(1,0) \\
\hat{\mathbf{f}}(0,1) \\
\hat{\mathbf{f}}(1,1)
\end{array}\right)=\left(\begin{array}{c}
\hat{\mathbf{u}}(0,0) \\
\hat{\mathbf{u}}(1,0) \\
\hat{\mathbf{u}}(0,1) \\
\hat{\mathbf{u}}(1,1)
\end{array}\right)
$$

The most interesting observation is that $\hat{\mathbf{G}}$ is a block diagonal matrix of $2 \times 2$ blocks where all blocks are symmetric and real. Therefore, it is convenient to solve Eq. (5.1) by the following steps:

1. $\hat{\mathbf{u}}=\mathcal{F}(\mathbf{u})$

2. Solve the block diagonal linear equation system $\hat{\mathbf{G}} \cdot \hat{\mathbf{f}}=\hat{\mathbf{u}}$ for $\hat{\mathbf{f}}$;

3. $\mathbf{f}=\mathcal{F}^{-1}(\hat{\mathbf{f}})$. 
In our case, we have a discrete system, so $\hat{\mathbf{G}}$ is expressed as the matrix given in Eq. (5.22) and $\mathbf{u}$ and $\mathbf{f}$ as a vector each. This approach is called Fourier Transform Traction Cytometry or short FTTC. In general, FTTC is considered an ill-posed problem and thus, regularisation is used [98]. However, in the following, it is shown that regularisation is not needed in our case when using PIV.

\subsubsection{On the Need of Regularisation in FTTC}

\subsubsection{Some Facts on Ill-Posed Problems}

In the following, the used definitions and derivations are based on the Refs. [27] and [53]. Regularisation to solve a problem is needed if the problem is ill-posed. So if we want to see if regularisation is necessary in our application of PIV, marker tracking and FTTC, we need to answer the question whether our problem is wellposed or not. When do we consider a problem as being well-posed?

The equation we intend to solve is of the form

$$
T x=y
$$

where $T$ is an operator from space $X$ to space $Y$ so that $x \in X$ and $y \in Y$. This problem is said to be well-posed if the following is true:

Definition. Let $X$ and $Y$ be normed spaces and $T: X \rightarrow Y$ a mapping. Then $T x=y$ is well-posed if:

1. for each $y$, there exists a solution (that is, $T$ is surjective);

2. the solution $x$ for every $y$ is unique (that is, $T$ is injective);

3. the solution $x$ depends continuously on $y$ (that is, $T^{-1}$ is continuous).

It is important to note that for each problem, multiple spaces $X$ and $Y$ may exist that can be used and contain all elements $x$ and $y$, respectively. Additionally, each of these spaces can furthermore have multiple norms that are meaningful. However, given that our data $y$ is disturbed by an error denoted by $\delta y$, the space $Y$ has to be chosen in such a way that also $\delta y \in Y$ holds true.

We later plan to solve the Boussinesq equation which has the form of an integral equation

$$
\int_{\Omega} K(t, s) x(s) d s=y(t)=T x(t)
$$


Depending on the structure of $K$, this equation is often ill-posed if one allows all kinds of errors on $y$. However, if choosing a suitable sub-space $Y_{0}$ with a corresponding norm, the ill-posedness may be avoided. Again, note that this subspace has to include not only $y$ but also $y+\delta y$.

\subsubsection{FTTC and Ill-Posedness}

Until now, we have not had any restriction on the data and errors. However, if we can assume any kind of a-priori knowledge about the data, it may be possible to construct a suitable space $Y_{0}$ (comp. examples in [27], p.12ff and [53], p. 11 and 14ff). But do we have any a-priori information? And does this information need to be in real space or could it be in Fourier space? Given that we do not know the 'real' displacement or the structure of the error, we could potentially find such information in Fourier space.

In order to simplify the notation let us assume that both $x$ and $y$ in Eq. (5.24) are only one-dimensional functions (and not two-dimensional as in Eq. (5.1)). Assume further that our spaces $X$ and $Y$ are $L^{2}(-\pi, \pi)$. Considering our errors in the data, we rewrite Eq. (5.23) as

$$
T(x+\delta x)=y+\delta y
$$

Applying the Fourier transform, we have

$$
\hat{T}(\hat{x}+\widehat{\delta x})=\hat{y}+\widehat{\delta y}
$$

with the corresponding spaces $\hat{X}=\hat{Y}=l^{2}$. The corresponding norm for the Fourier space is given by Parseval's identity,

$$
\|x\|^{2}=2 \pi \sum_{k=-\infty}^{\infty}\left|\hat{x}_{k}\right|^{2}=2 \pi\|\hat{x}\|_{l^{2}}^{2} .
$$

We know that a linear operator is continuous if it is bounded. The norm of a linear operator $A: X \rightarrow Y$ is defined as

$$
\|A\|=\sup _{x \neq 0} \frac{\|A x\|_{Y}}{\|x\|_{X}}
$$

It follows that

$$
\left\|T^{-1}\right\|=\left\|\hat{T}^{-1}\right\|
$$


Thus, if $T^{-1}$ is continuous, $\hat{T}^{-1}$ is so, too, and vice versa. Furthermore, we can conclude that the problem is equally well-/ill-posed in Fourier space as in real space. Hence, we continue to study our problem in Fourier space.

We know that our operator $T$ in Fourier space is given as a real, (block-)diagonal matrix, namely $\hat{\mathbf{G}}$. Thus, the eigenvalues of our matrix correspond to the singular values, here called $\lambda_{\mathbf{k}}$ with $k_{i}, i=1,2$ being the modes $\pm 1, \pm 2 \ldots$. Note that $\mathbf{k}=\mathbf{0}$ is excluded as we assume that we do not observe any rigid body motion.

A quantitative measure of the sensitivity of the solution of a linear system of equations is the condition number. Consider the linear system

$$
\mathbf{G f}=\mathbf{u}
$$

with a non-singular square matrix $\mathbf{G}$. The condition number of $\mathbf{G}$, $\operatorname{cond}(\mathbf{G})$, is then defined by ( [42], Chapter 2)

$$
\operatorname{cond}(\mathbf{G})=\|\mathbf{G}\| \cdot\left\|\mathbf{G}^{-1}\right\|
$$

Then, we have the bound

$$
\frac{\|\delta \mathbf{f}\|}{\|\mathbf{f}\|} \leq \operatorname{cond}(\mathbf{G}) \frac{\|\delta \mathbf{u}\|}{\|\mathbf{0}\|}
$$

where $\mathbf{G f}=\mathbf{u}$ and $\mathbf{G}(\mathbf{f}+\delta \mathbf{f})=\mathbf{u}+\delta \mathbf{u}$. Note that it always holds that $\operatorname{cond}(\mathbf{G}) \geq 1$. $\hat{\mathbf{G}}$ is a normal matrix and the condition number is calculated by determining the largest and smallest eigenvalue ( [42], Chapter 3). It can be shown that for each block, the eigenvalues are given by

$$
\begin{gathered}
\lambda_{\mathbf{k}}=\frac{2(1+\nu)}{E \cdot k} \cdot\left\{\begin{array}{l}
(1-\nu) \\
1
\end{array}\right. \\
\Rightarrow \operatorname{cond}\left(\hat{\mathbf{G}}\left(k_{1}, k_{2}\right)\right)=\frac{1}{1-\nu}=\{\nu=0.3\} \approx 1.43
\end{gathered}
$$

Note that $\nu$ describes the Poisson's ratio of the substrate, here taken to be 0.3 according to Ref. [64]. Hence, the condition number of $\hat{\mathbf{G}}\left(k_{1}, k_{2}\right)$ is close to 1 and independent of the modes. This shows that each individual block is insensitive to errors in the data. In order to find the condition number of $\hat{\mathbf{G}}$, we must divide 
the largest by the smallest eigenvalue providing us with

$$
\operatorname{cond}(\hat{\mathbf{G}})=\frac{1}{1-\nu} k
$$

Thus, our problem is only weakly ill-posed.

The block-diagonal structure of the matrix $\hat{\mathbf{G}}$ allows us also to define an effective condition number. It is obtained if modes $\mathbf{k}$ are neglected, e.g., if the corresponding right-hand side $\hat{\mathbf{u}}\left(k_{1}, k_{2}\right)$ is 0 . If $\hat{u}\left(k_{1}, k_{2}\right)=0$ for $|\mathbf{k}|>k_{\max }$, then

$$
\operatorname{cond}_{\mathrm{eff}}(\hat{\mathbf{G}})=\frac{1}{1-\nu} k_{\max } .
$$

Further below we will see that this is a very good approximation with a small $k_{\max }$ in our application.

In the regularised FTTC algorithm described by Sabass et.al [98], the system is nevertheless regularised by the Tikhonov regularisation of 0th order. What would happen to our system if we were to regularise with 0th order Tikhonov regularisation?

For a 0th order regularisation, the corresponding regularisation kernel is the identity matrix. The Fourier transform of the identity matrix is given by $\mathcal{F}(\mathbf{I})=\hat{\mathbf{I}}=\mathbf{I}$. Our regularised problem thus corresponds to (with $\alpha>0$ being the regularisation parameter)

$$
\hat{\mathbf{f}}_{\text {approx }}=\left(\hat{\mathbf{G}}^{T} \hat{\mathbf{G}}+\alpha \mathbf{I}\right)^{-1} \cdot \hat{\mathbf{G}}^{T} \cdot \hat{\mathbf{u}} .
$$

The eigenvalues $\lambda^{*}$ of $\left(\hat{\mathbf{G}}^{T} \hat{\mathbf{G}}+\alpha \mathbf{I}\right)^{-1} \cdot \hat{\mathbf{G}}^{T}$ are given by

$$
\lambda^{*}=\frac{\lambda}{\lambda^{2}+\alpha} \sim \frac{\frac{1}{k}}{\frac{1}{k^{2}}+\alpha}=\frac{1}{\frac{1}{k}+\alpha k} .
$$

So for $\alpha=0$ we have the original ones of $\hat{\mathbf{G}}^{-1}$ back. Observe that they behave like $C k$.

To study the influence of $\alpha$ on the eigenvalues, let us consider the limiting cases of Eq. (5.36). For easier understanding, let us simplify our problem to the onedimensional one as before. This means, we assume that all block entries in $\hat{\mathbf{G}}$ are one-by-one matrices instead of two-by-two. It then follows that our problem reads

$$
\hat{\mathbf{G}}\left(k_{1}, k_{2}\right) \cdot \hat{\mathbf{f}}\left(k_{1}, k_{2}\right)=\lambda\left(k_{1}, k_{2}\right) \cdot \hat{\mathbf{f}}\left(k_{1}, k_{2}\right)=\hat{\mathbf{u}}\left(k_{1}, k_{2}\right),
$$


such that

$$
\hat{\mathbf{f}}=\frac{1}{\lambda} \cdot \hat{\mathbf{u}} \quad \text { for all } k_{1}, k_{2} .
$$

Note that by our definition above, for the regularised case, $\lambda^{*}$ replaces $1 / \lambda$ from the un-regularised case. Thus, the solution for $\hat{\mathbf{f}}$ (and $\mathbf{f}$ ) scales with a scalar unit, the corresponding eigenvalue. The limit cases of the equation system is then given by the limits of $\lambda^{*}$ which in turn is given by the limits of the $\lambda^{\prime}$ 's of the un-regularised case as well as $\alpha$.

Considering Eq. (5.36), we can directly see that, for very large $\alpha$ compared to $\lambda^{2}$, the regularisation dominates the solution. Furthermore, we observe that the influence of $\alpha \geq 0$ is most notable for larger $k$ 's. Let us have a more detailed look at what this entails.

First, let us define the exact values of $\left(k_{1}, k_{2}\right)$. The single components of the wave vectors in the Fourier transform are calculated to be

$$
\begin{aligned}
& k_{1}=n_{\text {scal }} \cdot l, \quad-\frac{n}{2}+1 \leq l \leq \frac{n}{2}, \\
& k_{2}=m_{\text {scal }} \cdot l, \quad-\frac{m}{2}+1 \leq l \leq \frac{m}{2},
\end{aligned}
$$

where $(n, m)$ gives the number of modes in each direction and $\left(n_{\text {scal }}, m_{\text {scal }}\right)$ are scale factors given as $2 \pi / d, d$ being the interval length in the corresponding dimension (e.g, in our case, the width and height of our images, respectively, measured in the chosen pixel or SI units). It follows that the minimum and maximum values for each component are

$$
\begin{array}{ll}
k_{1, \min }=1 \cdot n_{\text {scal }}, & k_{1, \max }=\frac{n}{2} \cdot n_{\text {scal }}, \\
k_{2, \min }=1 \cdot m_{\text {scal }}, & k_{2, \max }=\frac{m}{2} \cdot m_{\text {scal }} .
\end{array}
$$

We directly observe that the vectors scale with the chosen units, $i$. $e$. we obtain different wave vectors for the same set of data depending on if we use pixel units or our actual SI-units, here $75.65 \cdot 10^{-9} \mathrm{~m} /$ pixel. The same is true for $\hat{G}$. However, the condition number is independent of the chosen units.

We now further conclude that the smallest and largest eigenvalues of $\hat{G}$ are given by

$$
\lambda_{\min }=\frac{2(1-\nu)(1+\nu)}{E \sqrt{n^{2} n_{\mathrm{scal}}^{2} / 4+m^{2} m_{\mathrm{scal}}^{2} / 4}}, \quad \lambda_{\max }=\frac{2(1+\nu)}{E \sqrt{n_{\mathrm{scal}}^{2}+m_{\mathrm{scal}}^{2}}} .
$$


Next, let us have a look at how the inclusion of $\alpha$ changes our solution for $f$. We see that according to Eq. (5.36), all eigenvalues to our problem are modified by the regularisation parameter. However, as our original system is only weakly ill-posed to begin with, we do not expect any notable changes in the result for sufficiently small $\alpha$. Let us now examine what values of $\alpha$ can be considered as small.

For $\alpha \rightarrow 0, \lambda^{*} \approx 1 / \lambda$. Thus, for a well-conditioned problem, we can set $\alpha=0$ and regain our original problem. The same result is seen if $\lambda$ is large compared to $\alpha$.

The next limit case to consider is the case where $\lambda \rightarrow 0$. Here, $\lambda^{*} \rightarrow 0$, contrary to the un-regularised case where $\lambda \rightarrow \infty$. This indicates that small $\lambda$ (i.e. large $k$ 's) are damped. Thus, the choice of $\alpha$ determines which $k$-values are damped. However, the damped $k$-values will always correspond to high-frequency noise. For too large choices of $\alpha$ compared to $\lambda$, the features that we plan to study are filtered and we loose information. We can hence determine an upper limit for $\alpha$ such that

$$
\alpha \ll \lambda_{\max }^{2}
$$

To test our theory, let us study our artificial data set of a contracting circle as described in Section 5.1. For five images, the circle contracts towards its centre before relaxing again via the same path to its original state (compare Fig. 5.1). We first calculate the velocity fields in units of pixel/s using PIV and then start the Lagrangian marker tracking, followed by the (regularised) FTTC calculation. To compare the results in dependence of $\alpha$, we always determine the maximum force during contraction, meaning the force detected at frame 5 . These calculations are done twice, once without any scaling (i.e. using pixel units) and once with the correct scaling to SI-units as given in our actual experiments. The results can be found in Tab. 5.1 and Tab. 5.2, respectively.

For our scaled system of the contracting circle $^{1}$, our upper limit for the choice of $\alpha$ implies that $\alpha<1.6 \cdot 10^{-19}$ is a reasonable choice. This is exactly what we observe in Tab. 5.2 where we regain the same order of magnitude as the un-regularised case at $\alpha=10^{-19}$ and similar values from $\alpha \approx 10^{-22}$. For the un-scaled system, the corresponding value is $\alpha<2.7 \cdot 10^{-5}$ which is in good agreement with the listed results in Tab. 5.1. The smaller values found for larger values of $\alpha$ denote the smoothing effect of a calculation where $\alpha$ dominates compared to $\lambda$.

\footnotetext{
$\overline{{ }^{1} E}=19 \mathrm{kPa}, \nu=0.3,256 \times 256$ nodes, $75.65 \cdot 10^{-9} \mathrm{~m} /$ pixel
} 


\begin{tabular}{|c|c|}
\hline Regularisation parameter $\alpha$ & Maximal Force $\left[10^{7}\right.$ a.u.] \\
\hline \hline $10^{-1}$ & $1.98 \cdot 10^{-4}$ \\
\hline $10^{-2}$ & $1.98 \cdot 10^{-3}$ \\
\hline $10^{-3}$ & $1.93 \cdot 10^{-2}$ \\
\hline $10^{-4}$ & $1.58 \cdot 10^{-1}$ \\
\hline $10^{-5}$ & $5.87 \cdot 10^{-1}$ \\
\hline $10^{-6}$ & $9.27 \cdot 10^{-1}$ \\
\hline $10^{-7}$ & 1.18 \\
\hline $10^{-8}$ & 1.34 \\
\hline $10^{-9}$ & 1.38 \\
\hline $10^{-10}$ & 1.39 \\
\hline $10^{-11}$ & 1.38 \\
\hline $10^{-12}$ & 1.38 \\
\hline 0 & 1.39 \\
\hline
\end{tabular}

Table 5.1.: The maximal force determined for the data set of a contracting circle, calculated in pixel units, $E=19 \mathrm{kPa}, \Delta t=1 \mathrm{~s}, 256 \times 256$ nodes.

The tables show another important phenomenon: The values for the forces stay bounded even if $\alpha=0$. Since our original problem is (weakly) ill-posed, we would expect that a regularisation, that is $\alpha>0$, is needed for reasonable results. So what is the explanation for the fact that no regularisation is needed?

In Fig. 5.5, the displacement is plotted against the absolute value of $k$ for each component in Fourier space, one frame of the previously used data set. We observe that for higher $k$-modes, the displacement values are rapidly decaying. We can model the decay by

$$
\hat{u}=c \cdot k^{-p}
$$

for a rough approximation of the speed of decay. Here, $c$ is a constant and $p$ is the decaying coefficient. We find for both examples a decaying coefficient of about 3 . Most interesting in the figure is the order of magnitude of $\left|\hat{u}_{x}\right|$ for high $|k|$ values. We see that the size of $\left|\hat{u}_{x}\right|$ for high frequencies is three orders of magnitude lower than the values for the dominant, lower frequencies. We can thus conclude that the influence of data stemming from noise is so small that regularisation is not necessary as we have seen previously and indicates that the previous steps of the algorithm are not amplifying noise unnecessarily. This can even be explained by a more detailed analysis of our problem. In fact, we have been able to show mathematically by construction a suitable space $Y_{0}$ that, for our algorithm, no regularisation is necessary. This is in contrast to the algorithm proposed in [98]. Details of the proof are found in the appendix, Section A.1. 


\begin{tabular}{|c|c|}
\hline Regularisation parameter $\alpha$ & Maximal Force $\left[10^{-9} \mathrm{~N}\right]$ \\
\hline $\begin{array}{l}10^{-1} \\
\end{array}$ & $6.47 \cdot 10^{-17}$ \\
\hline $10^{-2}$ & $6.49 \cdot 10^{-16}$ \\
\hline $10^{-3}$ & $6.48 \cdot 10^{-15}$ \\
\hline $10^{-4}$ & $6.48 \cdot 10^{-14}$ \\
\hline $10^{-5}$ & $6.49 \cdot 10^{-13}$ \\
\hline $10^{-6}$ & $6.47 \cdot 10^{-12}$ \\
\hline $10^{-7}$ & $6.48 \cdot 10^{-11}$ \\
\hline $10^{-8}$ & $6.48 \cdot 10^{-10}$ \\
\hline $10^{-9}$ & $6.47 \cdot 10^{-9}$ \\
\hline $10^{-10}$ & $6.48 \cdot 10^{-8}$ \\
\hline $10^{-11}$ & $6.48 \cdot 10^{-7}$ \\
\hline $10^{-12}$ & $6.48 \cdot 10^{-6}$ \\
\hline $10^{-13}$ & $6.48 \cdot 10^{-5}$ \\
\hline $10^{-14}$ & $6.49 \cdot 10^{-4}$ \\
\hline $10^{-15}$ & $6.47 \cdot 10^{-3}$ \\
\hline $10^{-16}$ & $6.47 \cdot 10^{-2}$ \\
\hline $10^{-17}$ & $6.39 \cdot 10^{-1}$ \\
\hline $10^{-18}$ & 5.67 \\
\hline $10^{-19}$ & 27.11 \\
\hline $10^{-20}$ & 49.25 \\
\hline $10^{-21}$ & 64.11 \\
\hline $10^{-22}$ & 75.61 \\
\hline $10^{-23}$ & 78.91 \\
\hline $10^{-24}$ & 79.12 \\
\hline $10^{-25}$ & 79.16 \\
\hline 0 & 79.16 \\
\hline
\end{tabular}

Table 5.2.: The maximal force determined for the data set of a contracting circle, calculated in SI-units, conversion factor from pixels to SI-units is 75.65 . $10^{-9} \mathrm{~m} /$ pixel, $E=19 \mathrm{kPa}, \Delta t=1 \mathrm{~s}, 256 \times 256$ nodes.

\subsubsection{Reduction of Image Noise}

We have now shown that regularisation is not necessary in the FTTC part of our algorithm after using PIV for the velocity field. Note that this holds true for any given data set of finite size which does not exceed a given information size $(e . g$. PIV windows). However, as demonstrated, even data sets as large as our artificial data set which, on average, contains up to four times the area than our real data sets of contracting blood cells, does not need any regularisation during FTTC. 
A

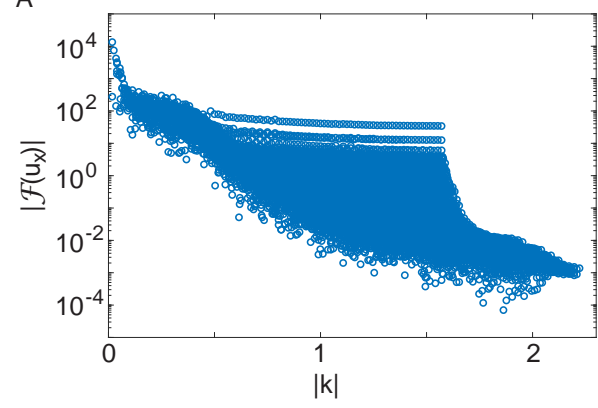

B

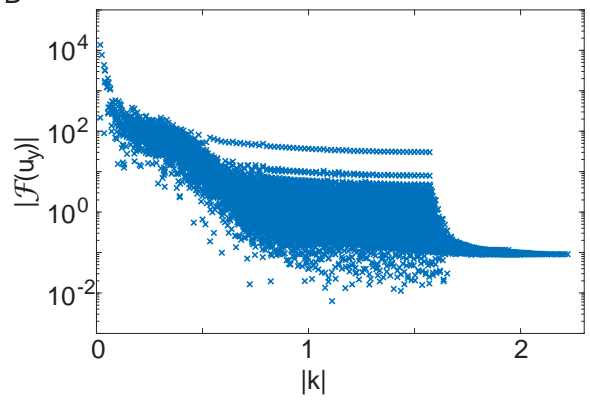

Figure 5.5.: Decaying $k$-values for a contracting circle at maximum contraction, frame 5. In $\boldsymbol{A}$, the Fourier transformed $x$-component of the displacement is given, in $\boldsymbol{B}$ the corresponding $y$-component. Decaying exponents for the exponential fitting model was approximately 3. The Fourier coefficients were calculated in pixel units.

This does not mean that we do not see image artefacts in the final traction force maps. Indeed, our results may, depending on the observed force pattern, show signs of Gibb's phenomenon. Fundamentally, the problem stems from the fact that for the Fourier transform done during FTTC, we assume a periodicity in the data which in fact is not present. To avoid the artefacts due to the nonperiodicity, we choose a different interpolation method when determining the local velocity at our markers' positions. Several different methods are tested, such as linear interpolation, Kriging interpolation [70], cubic interpolation and cubic spline interpolation. Examples of the result of different interpolation methods on real data can be found in Fig. 5.6. As the artefacts are due to spatially fast changing displacement field, here, the data of a platelet is taken instead of the continuous contracting circle.

As expected, linear interpolation works for areas of small changes. But, due to the discontinuity at the end points of the linear segments, this method fails for data sets which are highly variable in space. Kriging interpolation does not fail for any tested data set, however, studying the traction force magnitude maps in more detail, we observe some regular increases in force outside the actual contraction pattern. This is due to the fast decrease and increase of the underlying function that is used between neighbouring data points in Kriging interpolation. Measuring the distance between 'grid nodes' in the phantom force pattern shows that these nodes are located at the mid-points of the original PIV sub-windows. Similar effects are observed, to a lesser extend, in the cubic interpolation. 

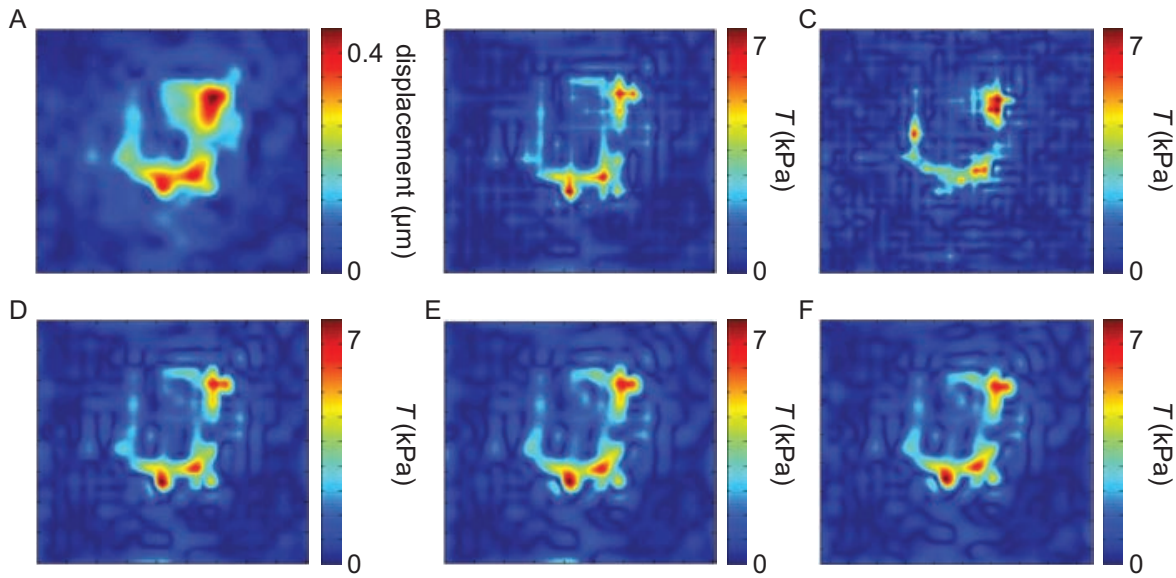

Figure 5.6.: A Displacement field of a contracting platelet. The corresponding traction force was calculated using different interpolation methods for the determination of the continuous velocity field: linear interpolation (B), Kriging interpolation $(\boldsymbol{C})$, cubic interpolation $(\boldsymbol{D})$, cubic splines $(\boldsymbol{E})$ and periodic cubic splines $(\boldsymbol{F})$.

Lastly, cubic splines are tested. While these work well within the inner part of our observed region of interest, boundary regions may cause problems as high noise forces are produced. These influence in particular the Fourier transform as we assume here that the data are continuous and periodically in space, particularly near the border regions. To avoid this problem, let us continue our PIV windows periodically in all directions, compare Fig.5.7. We use the three first and last columns and rows and assume them repeated at the corresponding opposite boundary of the image. The sub-windows at the corners are averaged over all four corners. Applying a cubic spline algorithm now yields largely undisturbed traction force data. We will hence call this interpolation method periodic cubic splines interpolation.

It is important to note that no matter which interpolation method is used at this stage, the overall force contained in the force map does not change its magnitude. The choice of interpolation method only reduces image artefacts.

\subsubsection{Total Force and Error Estimation}

In the end, we intend to compare different contractile cells to each other. This does not only include the spatial contraction behaviour as can be see in the traction force map but also their dynamics over time. To facilitate the direct comparison 

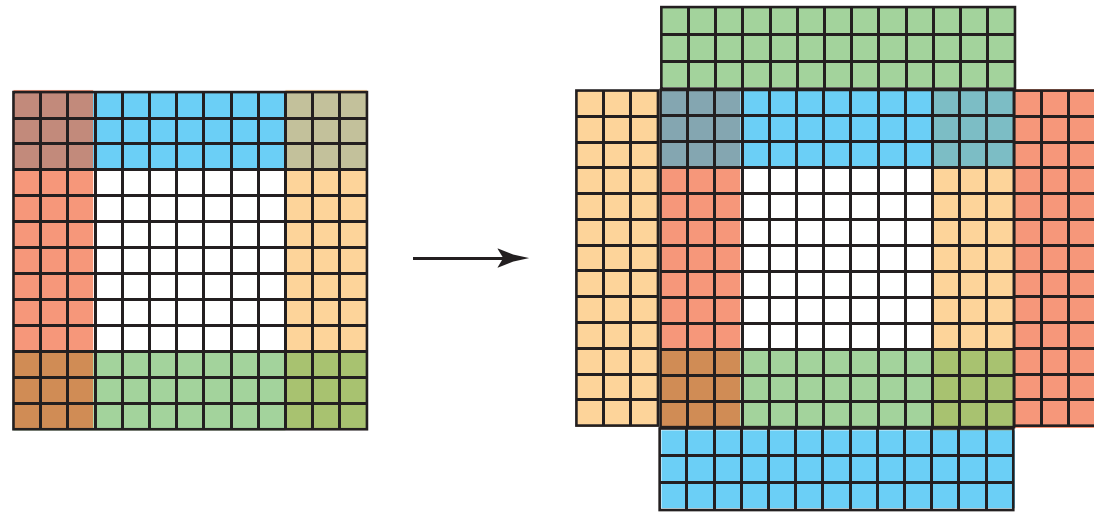

Figure 5.7.: The principle of periodic cubic splines. Assume the black grid denotes the set of PIV sub-windows, each small square containing one velocity vector. On each side, we now take the three outer columns (red and yellow) and rows (blue and green). We assume that they are repeated at their corresponding opposite side of our image as shown on the right image. The cubic splines are now calculated such that the additional windows at the border are also considered.

of different cells, we introduce the scalar entity total force, $F_{\text {tot }}(t)$ with the unit Newton defined as

$$
F_{\text {tot }}(t)=\int_{R O I}|\mathbf{T}(\mathbf{x}, t)| d A
$$

where $\mathbf{T}(\mathbf{x}, t)$ denotes the traction force at position $\mathbf{x}$ at the given time point $t$ within a defined region of interest, ROI. The ROI is positioned in such a way that all traction forces which can be associated to the actual contraction are included within while areas which are governed by noise of any kind are excluded. An alternative to the total force to compare cells to each other in a temporal fashion is the energy. Previously, the energy has been used to this end by, e.g. Oakes et al. [88]. The energy is given by

$$
E=\int \mathbf{T} \cdot \mathbf{u} d \mathbf{x}
$$

We are not employing the energy here as we could show that the angle between the calculated displacement vector $\mathbf{u}$ and the traction force $\mathbf{T}$ at regions with low forces was not 0 but in severe cases up to $180^{\circ}$ (Fig. 5.8 A and B). This observation was independent of the used algorithm to calculate the displacements and hence traction forces. While the energy in the end is not expected to yield a different result than the total force in terms of, e.g. stiffness dependency, we here employ the total force instead. 

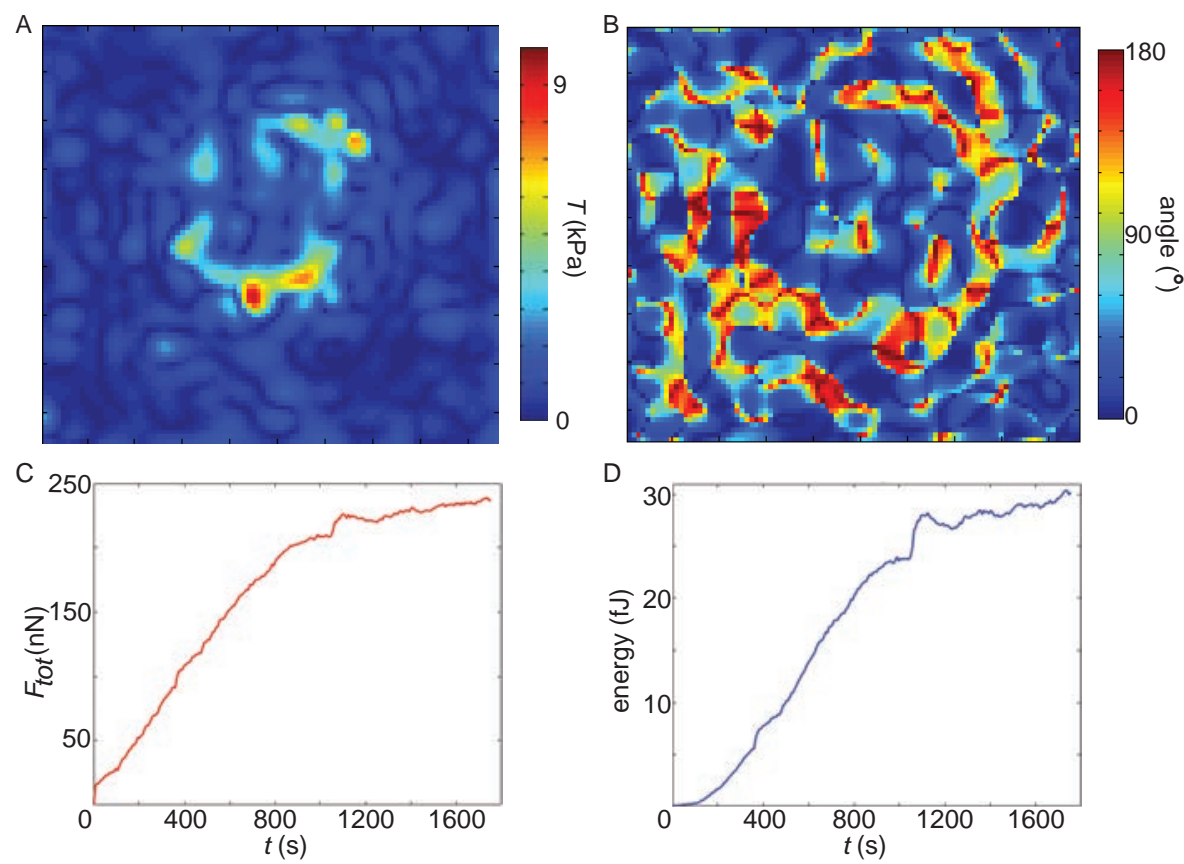

Figure 5.8.: A An example of a traction force map and the corresponding angles between them and the displacement vectors $(\boldsymbol{B})$. Where a high force is found, the angles are close to 0 while in between, they are up to $180^{\circ}$. This reflects in the conversion to the energy $(\boldsymbol{D})$ compared to the total force $(\boldsymbol{C})$ such that small features are overemphasised in the energy, as seen in the small jump at about $t=1100 \mathrm{~s}$. 
As already mentioned in the previous sections, all mathematical methods used here include uncertainties which add onto the inhered error due to the noise in the original images. It is impossible to measure each error and uncertainty by itself, however, the final cumulative error can be estimated. Theoretically, for non-motile contractile cells, the vectorial sum of all traction forces should be zero. However, this is not the case in our computations for the mentioned reasons. Though, the deviation from zero is utilised as an estimate for the error, $e(t)$, in the entire recording and analysis process by setting

$$
e(t)=\frac{\left|\sum_{R O I} \mathbf{T}(\mathbf{x}, t)\right|}{\sum_{R O I}|\mathbf{T}(\mathbf{x}, t)|}
$$

Note that the denominator is the numerical approximation of Eq. (5.40). We can now use the error estimate in Eq. (5.42) as a criterion of exclusion of which data to include in our further analysis. All cells over a defined error bound here are excluded.

\subsubsection{FTTC Using the Optical Flow Algorithm}

For the approach using the optical flow, a regular grid of displacements is needed again. Here, the irregular displacement field is interpolated onto a 2-by-2 pixel grid by using bivariate cubic splines. Contrary to the previously described algorithm based on PIV, for this algorithm, regularisation is necessary during the FTTC. Here, it is done by the so called Tikhonov regularisation of 0th order as proposed in Ref. [98]. To determine the regularisation parameter $\alpha$, the L-curve criterion used there does not yield reliable results in our application. Hence, Strong Robust Generalised Cross Validation $\left(\mathrm{R}_{1} \mathrm{GCV}\right)$ is used [72]. Lastly, the total force is calculated as described in Section 5.4.4. As for the optical flow algorithm above, this was implemented by D. Probst, University of Heidelberg.

\subsection{Force Dipole}

\subsubsection{Force Dipole Calculation}

We have previously (Section 3.2) introduced the concept of the elastic or force dipole $\mathbf{P}$ with the eigenvalues in two dimensions denoted as $D_{1}$ and $D_{2}$. We saw that for purely contracting cells with no forward movement, $\mathbf{P}$ is a symmetric matrix and the resulting dipole axes (= eigenvectors) perpendicular to each other. 
Here, we assume that our platelets do, indeed, not move in space. While this is a legitimate assumption, it does not follow that the calculated dipole matrix from the force data is always symmetric for any given time point. As already seen in previous sections, additional to any measurement noise, we introduce calculation noise during the evaluation. Thus, we often have some divergence from symmetry due to noisy vectors. To calculate the dipoles, we only consider the symmetric part of the calculated dipole matrix $\mathbf{A}$,

$$
\mathbf{A}=0.5 \cdot\left(\mathbf{P}+\mathbf{P}^{T}\right)
$$

Then, we employ the approximation

$$
q=\frac{\left|\lambda_{\max }(\mathbf{A})\right|}{\left|\lambda_{\min }(\mathbf{A})\right|}
$$

for $q_{\text {dipole }}$ as defined in Eq. (3.16).

Note that the dipole orientation and magnitude may still vary over time even if the traction force field remains stable overall due to the inherent noise level. Larger noise spots can be avoided by choosing a region of interest containing the cell in which to determine the dipole instead of regarding the entire, ideally infinite area. However, some noise remains, especially notable at time points where no clear force field is observed or just emerging. To avoid these time points, one can average the 'true' dipole quotient where appropriate. The question directly arises how one chooses the correct interval to average. In the following, two approaches are described.

\subsubsection{Dipole Quotient Averaging and Error Estimation}

To determine the average of the dipole quotient $q$, we have to exclude values of $q$ that are dominated by noise. This can be achieved either by calculating a weighted average using an error estimate or by using the variance of the quotient over time as a criterion of exclusion. Let us consider these approaches in detail.

\subsubsection{Weighted Average by Error Estimation}

To determine a weighted average of the dipole quotient, we use an estimation of the error as a weight. Here, the weight is given by the inverse error so values with higher error are counted less important than values of lower error. This approach 
has the advantage that we do not need to estimate which values to actually include in our calculation. But how do we estimate the error to be expected in our quotient?

Let us start by considering the dipole matrix $\mathbf{P}$. Ideally, $\mathbf{P}$ should be symmetric, however, due to noise, we do know that this is often not the case. But for any given matrix, we can divide it into two parts, a symmetric part and a skew symmetric part. The symmetric matrix is what we use to calculate the dipole as described in Eq. (5.43). The skew symmetric part is given by

$$
\tilde{\mathbf{A}}=0.5 \cdot\left(\mathbf{P}-\mathbf{P}^{T}\right)
$$

However, the matrices $\mathbf{A}$ and $\tilde{\mathbf{A}}$ are also disturbed by the error already contained in $\mathbf{P}$. Let us denote the exact unperturbed matrix by $\mathbf{A}^{*}$ (which is of course symmetric) and the error by $\epsilon$ between the matrices and thus the perturbation from the "real" result can be assumed to be

$$
\left\|\mathbf{A}-\mathbf{A}^{*}\right\|_{2} \approx\|\tilde{\mathbf{A}}\|_{2}=: \epsilon
$$

where $\|\cdot\|_{2}$ denotes the Euclidean norm. Here we simply assume that the size of the skew-symmetric part of the matrix $\mathbf{P}$ is an error indicator for the measurements. How does that error influence the quotient $q$ ? The quotient is defined as given in Eq. (3.16) where the major dipole axis is given by the largest eigenvalue of $\mathbf{A}$, $\lambda_{\max }$, and the minor dipole axis by the smallest eigenvalue, $\lambda_{\min }$. We do know that $\mathbf{A}$ contains some disturbance. The true quotient, $q^{*}$, is given by

$$
q^{*}=\frac{\left|\lambda_{\max }\left(\mathbf{A}^{*}\right)\right|}{\left|\lambda_{\min }\left(\mathbf{A}^{*}\right)\right|} .
$$

Let the difference between $q$ and $q^{*}$ be given by

$$
\delta=\left|q-q^{*}\right|
$$

We can draw two conclusions whose mathematical proof is given in the appendix, Section A.2:

- Under the condition that $\lambda_{\min (\mathbf{A})}>\epsilon$, the error can be estimated by

$$
\delta \leq \hat{\delta}=\frac{\epsilon\left(\lambda_{\max }(\mathbf{A})+\lambda_{\min }(\mathbf{A})\right)}{\lambda_{\min }(\mathbf{A})\left(\lambda_{\min }(\mathbf{A})-\epsilon\right)}
$$


- In the contrary case of $\lambda_{\min (\mathbf{A})} \leq \epsilon$, the error $\delta$ may become arbitrarily large. Hence, $\hat{\delta}$ gives an upper bound estimate of the real error in the dipole quotient. Note that the expression for $\hat{\delta}$ includes only computable quantities. If we now calculate $\hat{\delta}$ for each quotient over time, the inverse can be taken as a weight when averaging all values.

\subsubsection{Average Determined via the Variance}

As an alternative to the weighted average, we can also use the variance between consecutive values of the dipole quotient. Here, we use the knowledge that for $q_{\text {dipole }}$ values dominated by noise as is seen at very low forces yield highly fluctuating results within short time intervals. Thus, instead of taking all values and determine their influence on the average by their error, we exclude values where a high error is expected.

To determine the variance of the dipole quotient, the variance is calculated inside a moving window of a defined size. Afterwards, only values under a certain bound are included for the averaging. So we first have to determine an upper bound for the allowed variance in the dipole ratio. The most intuitive way is to estimate the error we expect in the variance. Thus, will it be possible to find a number of points where we can assume that the variance is mostly governed by noise, independent of the data set? Indeed, given our time resolution and an assumption that within the first few recorded time points, the forces are close to zero, we can define such a group of values. We here only include values of the ratio where the variance falls under a bound defined by the first variance values.

However, as we discussed above, there exist dipole values which inherently contains such a large error that the ratio does not yield any realistic result. To ensure that these values are discounted from our analysis, we identify them in such a way that all values where $\lambda_{\min }<\epsilon$ are excluded. $\epsilon$ is defined as given in Eq. (5.45). The variance is then only calculated over the remaining ratios. 



\section{Data Analysis}

In the following, the details of the data analysis are given. In Section 6.1, the analysis starting from the bead movies to the final force data is described. It is followed by the description of different methods used during post-processing interpretation in Section 6.2. Note that unless explicitly stated differently, all analysis was done using MATLAB R2009b and MATLAB R2017b .

\subsection{Calculation of Force Data from Bead Movies}

\subsubsection{Analysis Using the PIV Algorithm}

For the following analysis, the corresponding Matlab programmes are found in Appendix B. How the bead patterns are transformed into the traction force maps can be followed in Fig. 6.1. The starting point is given by the deformed substrate as noted in panel $\mathbf{A}$. For the same cell, the images of the membrane are depicted for three time points in panel $\mathbf{D}$.

Before the evaluation process, the data sets were inspected for out-of-focus images. During recording, changes in focus were observed that in the most severe cases reached up to about $1 \mu \mathrm{m}$ between successive images. While those were immediately counteracted on the microscope by modifying the focus, larger jumps resulted in an unusable, blurry image of the beads. As the used algorithm relied on the information of successive images, an alternative was included at these time points were direct comparison was not possible. To still hold up the time sequence, the blurry image was exchanged by a single black frame. These frames were handled differently from the other frames as will be described later.

To start out, as mentioned in Section 4.4, due to the fact that platelets are very light sensitive, only minimal illumination was used. This resulted in bead images of comparably low contrast although the beads themselves were photo-stable over time. To facilitate the PIV analysis, the contrast of all images was increased

\footnotetext{
${ }^{1}$ MathWorks Inc., Natick, MA, USA
} 


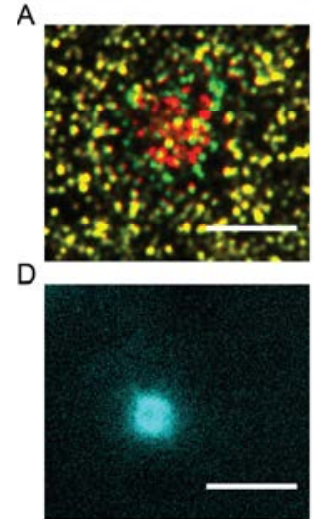

$\mathrm{t}=0 \mathrm{~min}$
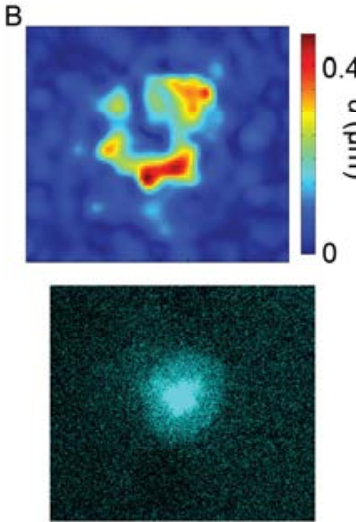

$\mathrm{t}=10 \mathrm{~min}$
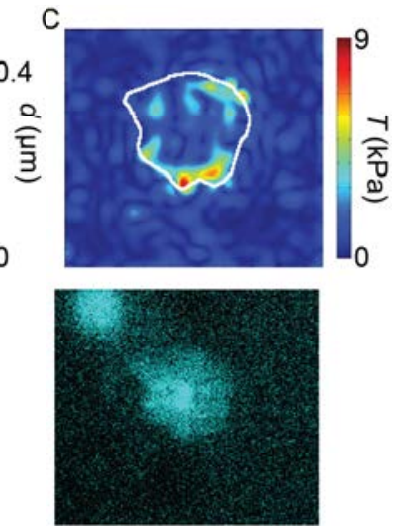

$\mathrm{t}=22 \mathrm{~min}$

Figure 6.1.: An example on how the deformation of the substrates translates to the forces. A The overlay of the relaxed (green) and contracted (red) bead patterns. The calculated displacements and traction forces are shown in panels $\boldsymbol{B}$ and $\boldsymbol{C}$, respectively. The white contour over the traction force denotes the cell outline at this stage. For the first, last and intermediate time points, the cell images are added in $\boldsymbol{D}$. The progressively lowering of the dye signal can be observed. Scale bars: $5 \mu \mathrm{m}$. Part of the image is partially taken from [36].

by applying a non-linear contrast enhancer (compare Appendix B.2). The entire filtering procedure is depicted step-by-step in Fig. 6.2, starting with the original image of high noise level (A).

For the non-linear enhancer, the histogram of each image was remapped to the interval $[0,1]$ such that the lowest intensity was assigned 0 and the highest measured intensity in each image was assigned to 1 (Fig. 6.2 B). Subsequently, the new intensity distribution was redefined such that $s \rightarrow s^{p}$ for $s \in[0,1]$ where $p>1$ (Fig. $6.2 \mathrm{C}$ ). As a result of the exponentiation, values close to 0 and thus interpreted as noise were repressed while values close to 1 and thus seen as signal were enhanced. At the same time, the lower and upper boundary of 0 and 1 remained constant. $p$ had to be chosen individually for each data set depending on the perceived contrast in the movie. The higher the noise level, the higher $p$ had to be chosen. Overall, analysable data sets had a $p$ value between 1.7 and 2.8. To remove remaining noise, a Wiener filter of size 3-by-3 pixels was applied. Lastly, the images were converted back to 16 bit (Fig. 6.2 D). Apart from the focus shifts mentioned earlier, small changes in FOV could also be observed over time. They were corrected by cross-correlation in Fourier space as described in Ref. [33] (compare Appendix B.3). 
A
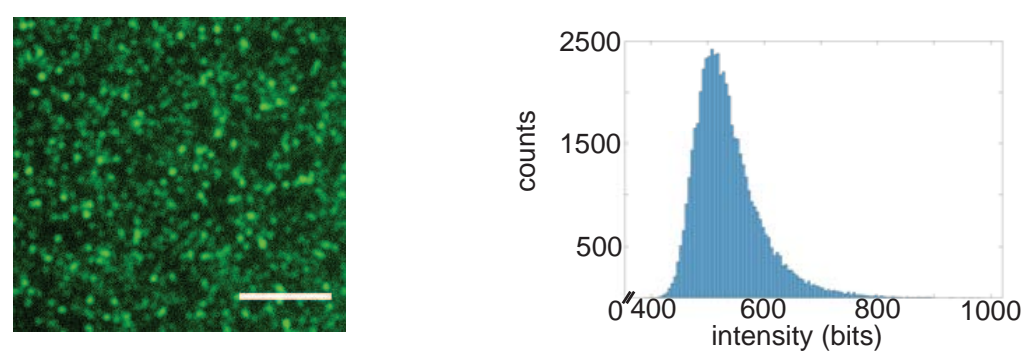

B
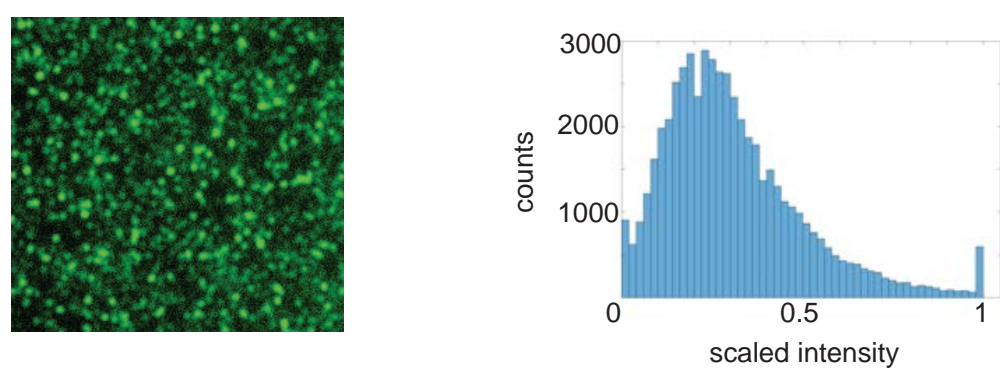

C
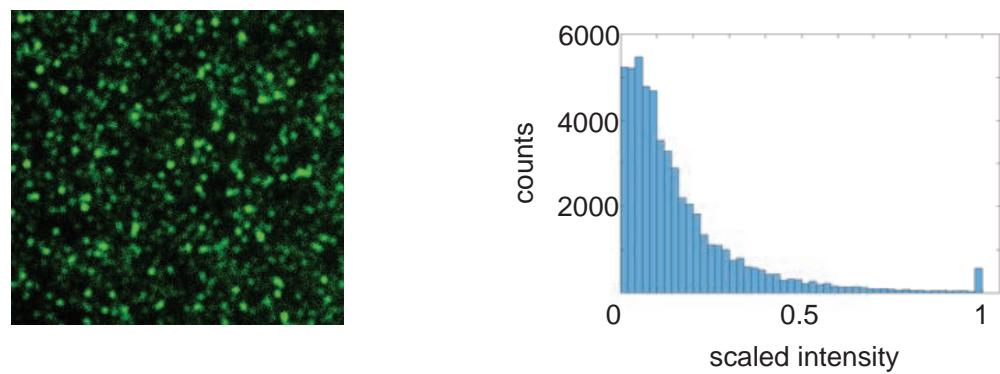

D
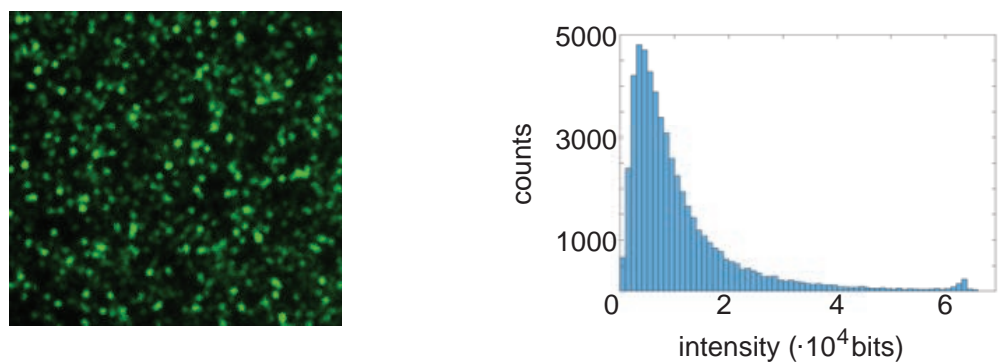

Figure 6.2.: The filtering process step-by-step. On the left hand side, the current image of the fluorescent beads is shown, on the right hand side the corresponding histogram. A The unfiltered image recorded in 16 bit. As can be seen by the noisiness in the image and the narrow and low intensity spectrum, the image has both a low intensity and contrast. $\boldsymbol{B}$ The unfiltered image is mapped onto the interval $[0,1]$ and adjusted to span the entire spectrum. $\boldsymbol{C}$ The intensity distribution is scaled with the exponent $p=1.7$. This greatly increases the contrast between background and beads. $\mathbf{D}$ Lastly, a Wiener filter is applied and the image is mapped back onto 16 bit. Scale bar: $5 \mu \mathrm{m}$. 
Following the image de-noising and contrast enhancing part, the PIV algorithm was used as described previously in Section 5.2.1, using the MATLAB toolbox $\mathrm{mPIV}^{2}$ (compare Appendix B.4). Specifically, first, a coarser grid of windows was used with a edge size 32 pixels or 40 pixels and kept fixed for the entire recording. Given the objective magnification as well as the camera pixel size, this corresponded to a window area of $5.9 \mu \mathrm{m}^{2}$ to $9.2 \mu \mathrm{m}^{2}$. The choice of starting grid size was made by studying the degree of displacement of the beads; for larger displacements between successive images, a larger window size was chosen. Note that neighbouring windows overlapped by $50 \%$. After the first round of velocity field approximations at the coarser grid, the edge length of each window was halved and the PIV algorithm was repeated on this finer grid, taking the first estimation as a guideline for the expected velocity values. Using a median filter, velocity vectors differing considerably from neighbouring vectors, were removed and replaced by local interpolation using Kriging interpolation. Furthermore, windows without unique definable velocity were also interpolated using the neighbouring windows. Whether a velocity was considered a unique definable velocity was here determined by comparing the highest and second highest peaks in the cross-correlation matrix during PIV. They had to differ significantly from each other for the velocity of the highest peak to be considered correct within the considered window. Using this approach, it was possible to gain a spatial resolution in the velocity field of down to $600 \mathrm{~nm}$. Here, the spatial resolution was not defined as a visual resolution between resolvable features but as the distance of neighbouring PIV windows as used earlier in e.g. Ref. [98]. This means that we considered the spatial resolution to be a resolution of resolvable velocity information.

As mentioned previously, blurred images of the beads were replaced by single black frames. Assuming that this was the case at time point $t_{j}$, a comparison between the image pairs $t_{j-1}$ and $t_{j}$ as well as $t_{j}$ and $t_{j+1}$ using differential PIV was not possible to carry out. Instead, the velocity within this time interval was assumed to not differ significantly from the previous time point where a measurement was possible. Thus, the velocity field in the time interval of interest was set to be the velocity calculated between $t_{j-2}$ and $t_{j-1}$. Note that this approximation was only valid at time points were either no velocity was detected or very little variation over time could be observed.

Before continuing with the Lagrangian marker tracking as derived in Section 5.2.2 (compare Appendix B.5), we adapted our velocity fields slightly. In some instances, the PIV algorithm caused erratic velocity vectors at the borders of the studied

\footnotetext{
${ }^{2}$ http://www.oceanwave.jp/software/mpiv
} 
frame. As we later interpolated the velocity fields using periodic cubic splines, the existence of such vectors meant that we included randomly oriented vectors in our calculation close to the boundaries. Furthermore, considering our drift correction given above, for larger drifts in FOV, beads appeared on one side of the image that previously were located at the opposite border. Hence, the first row and column of PIV windows were discarded for the static experiments.

For the flow experiments with trice the observation time, the drift in FOV was more dramatic than in the static case. Here, much larger ROIs were chosen from the movie to later be able to cut the first two rows and columns or, in a few rare cases, even the first three rows and columns. Note that this did not influence whether a data set was analysable or not. One had only to choose platelets with a sufficiently large distance to its closest neighbour and enlarge the ROI accordingly. For the set of Lagrangian markers, a regular grid of 2-by-2 pixels was chosen which was also directly employed for the transformation into Fourier space in the FTTC later on. An example of such a displacement field is found in Fig. 6.1 B. For the FTTC, a Poisson's ratio of $\nu=0.3$ was used [64]. The resulting traction force map is exemplary depicted in Fig. 6.1 C.

Lastly, after the calculation of the traction force field, a ROI was chosen to determine the total force over time as given by Eq. (5.40). Simultaneously, the error in the force was estimated in the same ROI as defined in Eq. (5.42). As can be seen in Fig. 6.3, in the fully contracted state, the relative error fluctuated around a near constant value, exhibiting a stable level of error. During initial contraction, the relative error stabilised while it increased again during relaxation. Only contraction data where the relative error after initial contraction was maximum $10 \%$ were included in the further analysis. Note that this corresponded to an absolute error of a few $\mathrm{nN}$, as seen in the bottom graphs in Fig. 6.3.

\subsubsection{Analysis Using the Optical Flow Approach}

The optical flow algorithm was programmed in Python 2.7.13 using the existing optical flow routines from OpenCV ${ }^{3}$. Before the analysis, the movies were downsampled from the recorded 16 bit to 8 bit images. To correct for the drift in FOV, a box of 50-by-50 pixels was placed within a corner where no displacement due to cell contraction was observed. All calculated bead displacements within this corner were then subtracted from the entire displacement field. The analysis

\footnotetext{
${ }^{3}$ Open Source Computer Vision Library, https://github.com/itseez/opencv

${ }^{4}$ The programme was implemented by D. Probst, University of Heidelberg.
} 
A
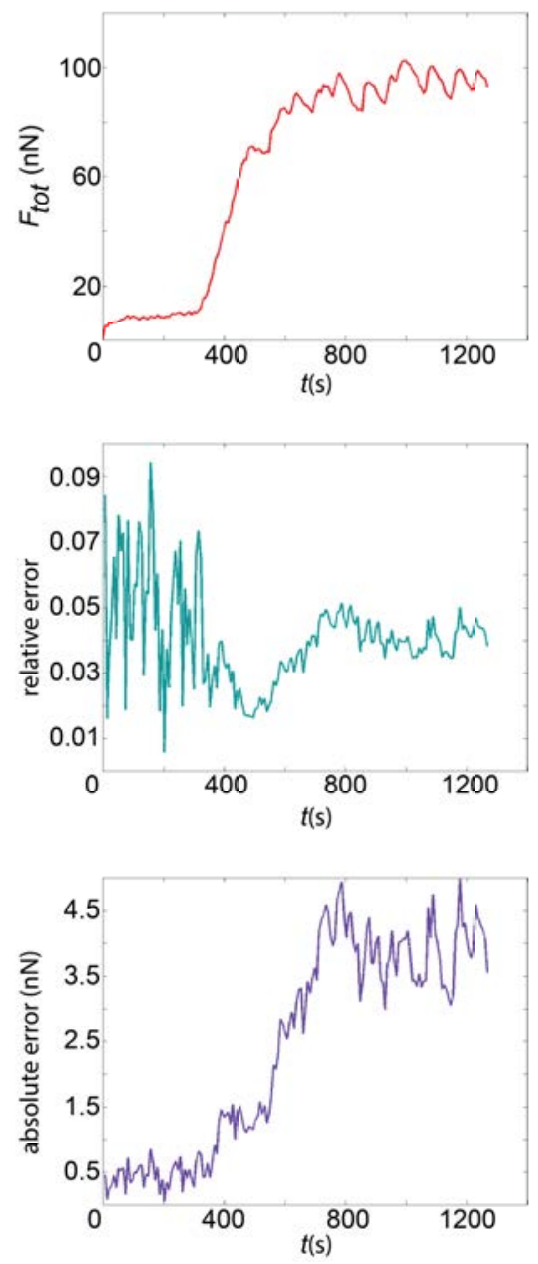

B
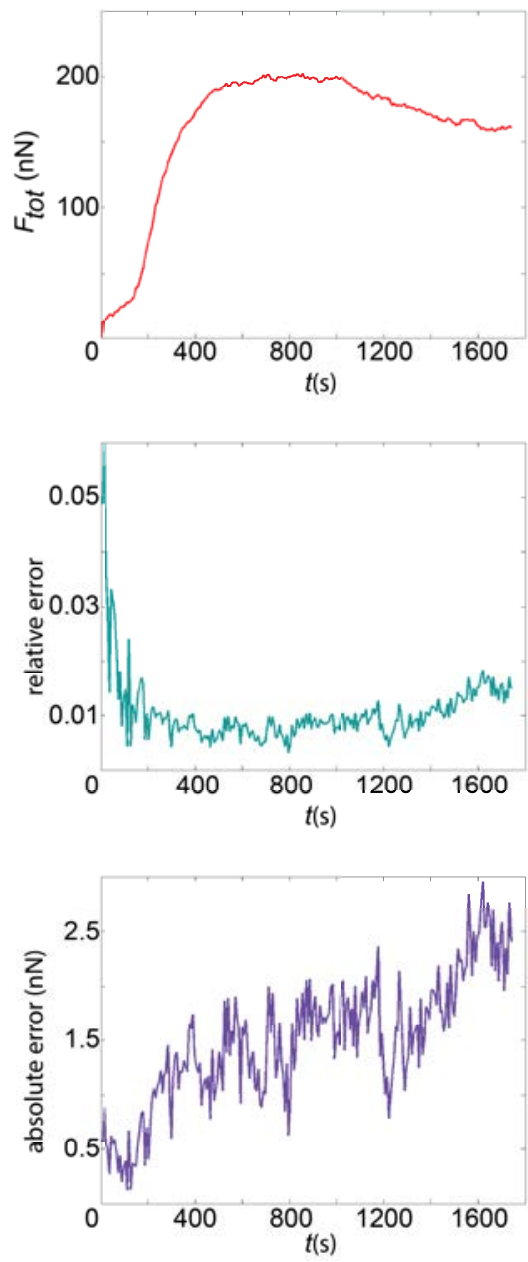

Figure 6.3.: Total force development of two platelets over time with the corresponding estimated errors. In panel $\boldsymbol{A}$, a oscillating platelet is shown. The relative error stabilises in the contracted state at about $700 \mathrm{~s}$ as seen in the second row graph (turquoise). In panel $\boldsymbol{B}$, the same stabilisation of the relative error is seen, starting at about $400 \mathrm{~s}$. During relaxation, the error increases again. The overall absolute error shown in last row (purple) stays at a few $\mathrm{nN}$. The figure is taken from Ref. [36]. Both cells were recorded on $19 \mathrm{kPa}$ substrates. 
was conducted as described in Section 5.3. Between beads, a minimum distance of 3 pixels was assumed and a maximum of 1000 features were tracked. For the sub-windows around the beads, an initial size of 64 -by-64 pixels were used. As mentioned previously, displacements near the image border were set to 0 by using a Tukey filter with an constant $\beta=0.2$. Furthermore, for the regularisation during FTTC using $\mathrm{R}_{1} \mathrm{GCV}$, the tuning parameter was chosen to be $\gamma=0.95$. The total force was calculated as previously done for the PIV algorithm.

\subsection{Post-processing Analysis}

\subsubsection{Determination of Final Spread Area}

Each contracting platelet was not only analysed towards their exerted force but also for their fully spread area. For the static experiments, as mentioned in Section 4.1, the membrane dye CellMask Deep Red was used. However, it was observed that this dye is not photostable over longer times as shown in the decreasing contrast in the image sequence in Fig. 6.1 D. While this was taken into account during isolation and the staining by keeping those times as short as possible without effecting the actual experiment, the dye still grew steadily fainter during recording. Furthermore, some dye remained in the solution thus yielding an additional fluorescent background signal that disturbed the image quality, especially as the light exposure was comparably low. On the other hand, the middle part of the cell where the granules clustered together gave of a much higher signal than the remaining membrane. This made the determination of the platelet size difficult. Automatic algorithms such as e.g. the Canny edge detection were tried as it had been shown to work on similar images [102]. However, the contrast between background and membrane was found to be too low for these algorithms to be usable.

For cells that did not bleach too fast, a semi-automated analysis using logarithmic images of the cells in Fourier space was employed. An example for this approach can be found in Figure 6.4. The image of the platelet was noisy and in the upper left corner, another stained cell was floating by when the snapshot was taken. Studying this particular image in Fourier space showed large variations in the Fourier coefficients, spanning six orders of magnitude. Transferring the Fourier spectrum of the image into a logarithmic scale revealed a sharp peak in the low frequencies but no indication of clear borders. Hence, high frequencies were cut to avoid the noise. This, however, resulted in blurry, smeared out images as seen 
in panel $\mathbf{B}$. This mask was too large but was used as a guideline for the overall outline. Slightly shrinking the contour obtained by the logarithmic image and addition of filopodia and smaller details by hand using ImageJ yielded a suitable mask for the spread platelet.

Yet, this approach did only work for images of platelets were the membrane dye was not noticeable bleached and some contrast was observed by eye. This was generally not the case and while the membrane was seen by eye inspection, even semi-automated algorithms failed. On the other hand, it was observed that the membrane exhibited only very minor changes in the later time points after initial spreading. Thus, to determine the final spread area for all cells, the outline was drawn by hand in ImageJ for one of the last five cell images and then compared to the remaining four snapshots for possible minor adjustments. The area was directly calculated given the known conversion rate between pixel size and physical length. This procedure was repeated multiple times for the same image to reduce human error.

For the flow experiments, the platelets were not stained due to the short life time of the dye. Instead, bright-field images were taken. As the fully spread cells were very thin especially towards the outer borders, the full membrane was mostly seen with a bright halo around it, making edge detection algorithms again not suitable. Hence, again, the hand-drawn version was used as done for the stained platelets.

\subsubsection{Determination of Primary Oscillating Frequency}

To be able to distinguish oscillatory contractile behaviour from other behaviours, a Fourier analysis was used. Here, we distinguished between two to three time intervals of contraction: the initial contraction, the contracted state and, in some cases, the force relaxation. The initial contraction was defined as the time interval in which the force rapidly increased after adhesion. Taking the examples in Fig. 6.5, first row, this would correspond to the time between approximately $350 \mathrm{~s}$ and $500 \mathrm{~s}$ for the platelet depicted in panel $\mathbf{A}$ and, for the platelet of panel $\mathbf{B}$, the time between about $200 \mathrm{~s}$ and $500 \mathrm{~s}$. The contracted state followed directly after the initial contraction. Some cells, as e.g. the platelet in panel $\mathbf{B}$, showed a third interval of relaxation, here starting at about $1000 \mathrm{~s}$. In some few cases not shown here, only the first and third time interval were observed. Oscillations occurred in the second contraction interval and occasionally the third interval. 


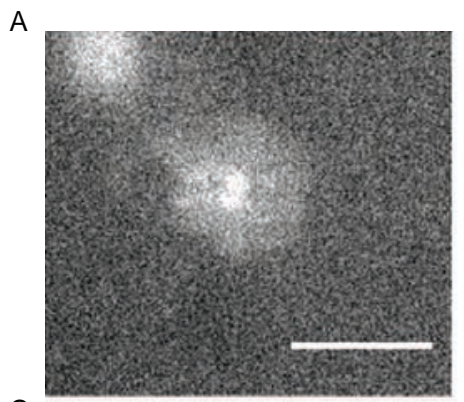

C

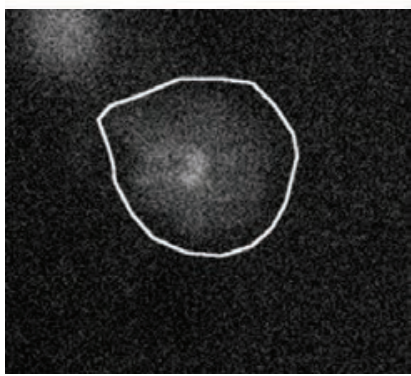

B

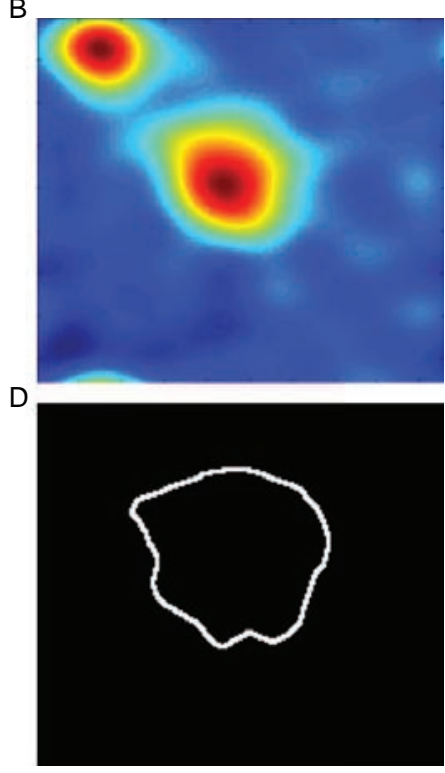

Figure 6.4.: A shows an original snapshot of a stained platelet membrane after about 20 min of recording. The brighter spot in the upper left corner is another cell floating by. In panel $\boldsymbol{B}$, the filtered, logarithmic image is depicted. $A$ heat map was chosen to better visualise the differences in intensity. The edge was then be detected where sharp changes between light blue and dark blue was seen when the upper cell was ignored. The calculated cell contour overlayed with the original cell image is given in panel $\boldsymbol{C}$. The darker image is due to autocontrasting. The final platelet outline is shown in panel $\boldsymbol{D}$. Here, the contour from $\boldsymbol{C}$ was shrunk and filopodia added in accordance to the image of the cell membrane. Scale bar: $5 \mu \mathrm{m}$. 
A
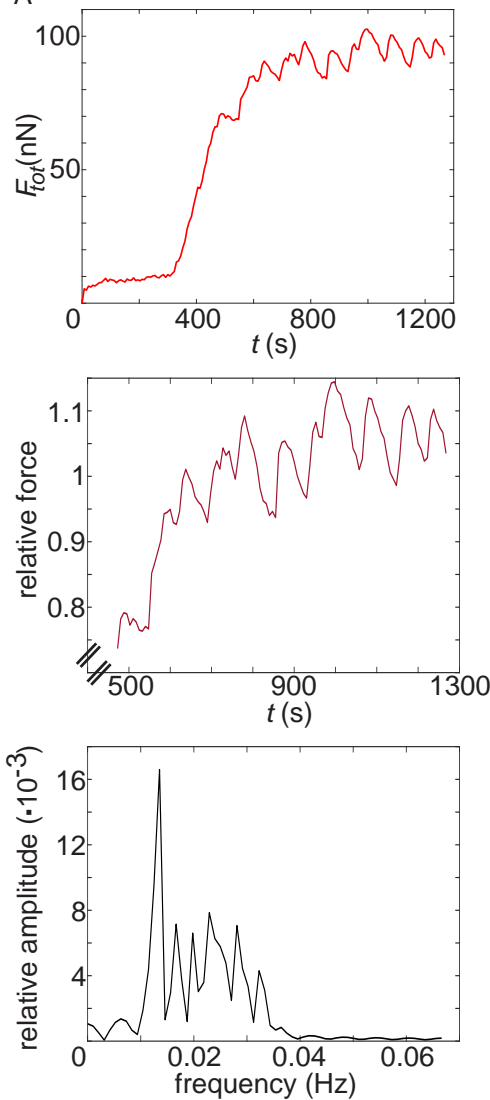

B
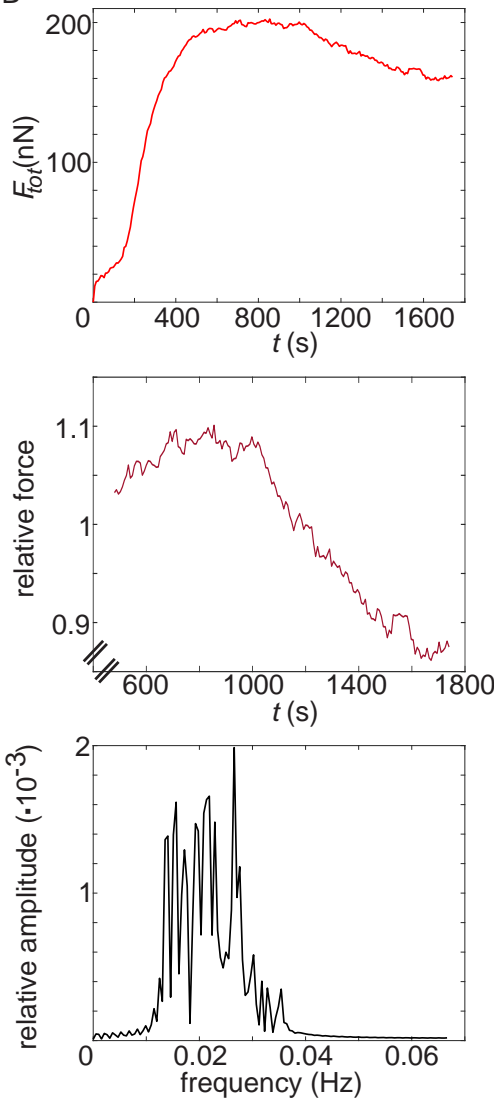

Figure 6.5.: Two examples to demonstrate the analysis process to determine the primary oscillatory frequency. In $\boldsymbol{A}$, an oscillating platelet is shown, in $\boldsymbol{B}$ a non-oscillating cell. From the total force (upper row), the interesting time interval is determined and the relative force calculated (middle row). For both cells, the time interval was taken to start at about $500 \mathrm{~s}$. From the Fourier spectrum (lower row), the dominate frequency, denoted by the highest peak in the spectrum, is derived. Both platelets were recorded on $19 \mathrm{kPa}$ substrates.

Given that the interesting part showing oscillations was found after initial contraction, the Fourier analysis was only conducted on this later interval. In cases where a second force plateau was reached after relaxation, the second plateau was excluded. To ensure that the study of oscillation frequency was not biased towards the mean in force, the total force was first divided by the mean force, compare Fig. 6.5, second row. This ensured that oscillations of $10 \mathrm{nN}$ amplitude were not treated equally for platelets reaching forces of, e.g, $50 \mathrm{nN}$ and $200 \mathrm{nN}$. 
Next, frequencies not corresponding to physical oscillations were filtered by a digital filter. These frequencies included very low frequencies representing the average force development as well as very high frequencies corresponding to noise. It could be shown that a Butterworth band-pass filter suppressing all frequencies below $10 \mathrm{mHz}$ as well as all above $35 \mathrm{mHz}$ worked well towards these specific requirements. These boundaries were chosen by estimating the oscillating frequency of clearly classified platelets and allowing for a broad spectrum of frequencies around this estimate. The remaining force graph was then transformed into Fourier space by Fast Fourier Transform (third row). The dominant frequency of each data set was then defined as the frequency displaying the largest relative amplitude in the spectrum (for $\mathbf{A}$ about $13 \mathrm{mHz}$ and $\mathbf{B} 27 \mathrm{mHz}$ ). In general, an oscillating platelet was expected to exhibit a considerable higher amplitude at the dominant frequency then a non-oscillating cell.

\subsubsection{Determination of Force Dipole Moment Orientation and Magnitude}

For all force experiments, both static and in flow, the force dipole moment was calculated. The definition of the force dipole may be found in Refs. [56, 104, 126] and the specific mathematical derivation used in this thesis in Section 5.5. The following analysis discription is depicted in Fig. 6.6.

As previously done for the calculation of the total force, an ROI was chosen in which the force dipole was determined according to Eq. (3.14). The choice of the ROI was important as the theory behind the force dipole assumes an infinitely large area over which to integrate containing a force field that converges towards zero. However, statistical noise was observed in the force fields. To avoid this noise, the ROI were chosen as small as possible, contradicting to some extend the assumption of a infinitely large area. A compromise had to be found here. To help the decision on a reasonable size and position of the ROI, several criteria were checked.

The ROI was chosen on the traction force map with the highest single value of traction force magnitude. Here, it was easiest to estimate the largest expansion of the traction forces and the possible position of noise. Sometimes, the position of noise was seen better on the last image of the data stack, and was additionally consulted. In total, at least 10 different sizes and positions were tested per data set to study the deviation between them. Generally, the calculated result of the 
A

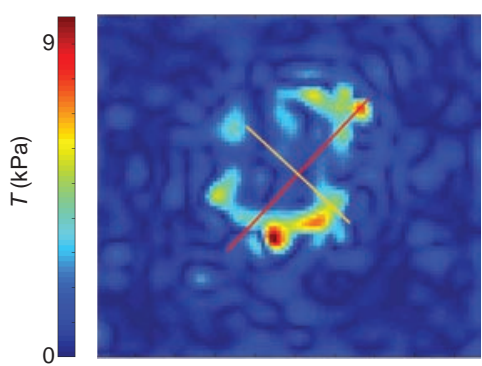

C

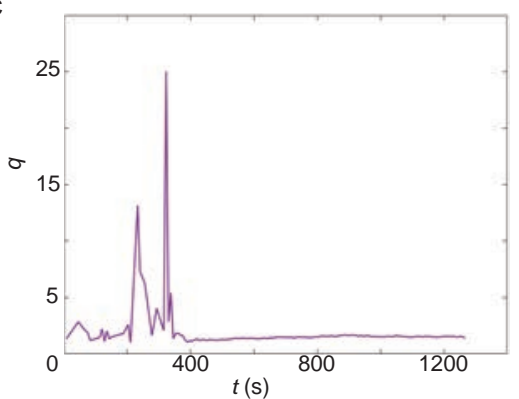

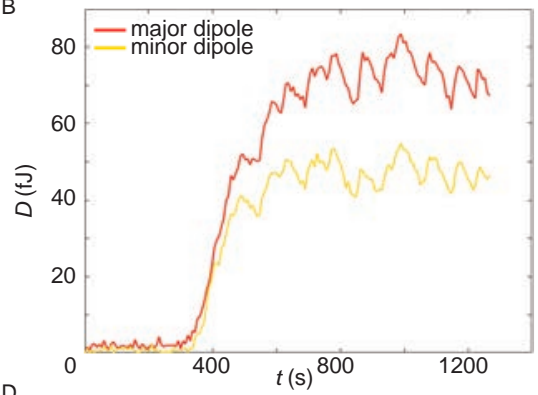

D
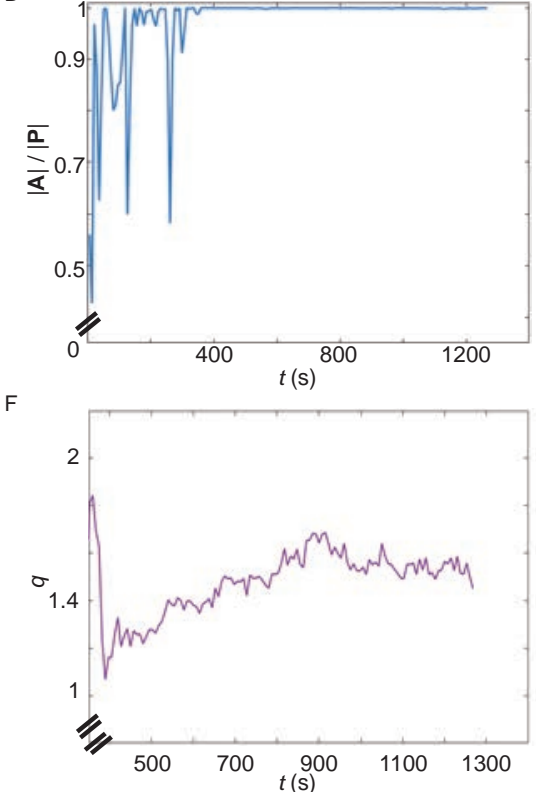

Figure 6.6.: A An example of the orientation of the major (red) and minor (yellow) dipole axis. Underlying the axis is the corresponding traction force pattern. The orientation of the major dipole axis is governed by the hot-spots in the traction force. Note that the lengths of the axes are scaled according to their magnitude. The corresponding dipole magnitudes over time can be seen in panel $\boldsymbol{B}$. The dipole moments develop largely in synchrony. The quotient $q$ between major $\left(D_{1}\right)$ and minor $\left(D_{2}\right)$ dipole moment is depicted in panel $\boldsymbol{C}$. As soon as a noticeable dipole moment develops $(t \approx 400 \mathrm{~s})$, the quotient stabilises. Simultaneously, the quotient between the norm of the symmetric dipole matrix $\mathbf{A}$ and the real dipole matrix $\mathbf{P}$ stagnates close to 1 . A value of 1 here corresponds to a real, symmetric matrix, thus no disturbance of the dipole due to noise. $\boldsymbol{E}$ By calculating the variance over $q$, the time interval can be determined at which the quotient is stable. Note that this interval corresponds to the time at which a clear dipole moment is observed in panel $\boldsymbol{B}$. The quotient $q$ at the calculated time interval is shown in panel $\boldsymbol{F}$. As can be seen, the changes in $q$ are comparatively small. The averaged quotient for this example was calculated to be 1.49. The images are partially taken from Ref. [36]. 
dipole quotient $q$ determined as given in Eq. (3.16) did not vary largely between similar ROIs. Large differences at this point suggested either a too large ROI including noise, or too small excluding single traction forces.

Next, the deviation of the dipole matrix from symmetry was studied (Fig. 6.6 D). As mentioned previously, we only considered the symmetric part of the dipole matrix as no movement during contraction was observed. This meant of course that the calculated dipole moments and their corresponding quotient were determined under the assumption of a slightly different force field. Hence, it was of interest to study the difference between the computed matrix and its symmetric part. To this end, the quotient of the matrix norms was calculated. Here, a quotient of 1 equalled to the calculated matrix being symmetric. Both the highest value of this quotient as well as the mean were kept as close as possible to 1. Indeed, for all data later presented, the highest value was always 1 and the mean quotient did not deviate more than $2.5 \%$.

Another interesting measure for the choice of the ROI were the actually included values in the averaging of the dipole quotient (Fig. 6.6 E). This quotient was used as a measure for isotropy and represents a physical characteristic which was used to compare cells to each other. As described in detail in Section 5.5.2, if the forces were very low, the noise dominated the quotient, making an exclusion measure essential. Two different versions were already presented in Section 5.5.2. Both measures yielded the same results in the averaged dipole quotient. Here, the approach of the moving variance including the estimation of stability was chosen. Values denoted as unstable by the criterion $\lambda_{\min }<\epsilon, \epsilon$ as defined in Eq. (5.45), were set to $\mathrm{NaN}$ and disregarded in the following analysis. This had the advantage that we were able to visualise all values of low enough error that were considered in the averaging and used this as another aid to determine a good ROI. No values of $q$ were excluded where a definite force was observed while values with no force were excluded. Choices of ROI where this was violated were not considered. For this analysis step, a moving window for the variance with a size of 17 values was taken. In the beginning and end of data sets where less then 17 values were available, less values were used and the variance calculated accordingly. To establish a boundary as to when to exclude values due to too high variance, the variance of the first three time points was considered. During this time, equivalent to the first $15 \mathrm{~s}$ after attachment, generally, no force was detected. Thus, the variances determined here represented an expected bound for the variance for images of pure noise. Time points of variances of less or equal to $10 \%$ of this bound were considered. 
Lastly, the orientation of the dipole moments compared to the corresponding force fields were inspected (Fig. 6.6 A). Generally, a low number of spots of very high force were observed. These dominated the orientation of the force dipole. Thus, plotting the dipole axes over the force field showed the major axis acting as a connection line between these spots, especially well seen on highly polarised platelets with only two force maxima. This agreement of the major dipole axis orientation to the force maxima was also taken under consideration for the choice of a reasonable ROI. The minor dipole axis was always perpendicular to the major axis due to our assumption of a symmetric dipole matrix.

\subsubsection{Angle of Flow Direction vs Angle of Major Dipole Axis}

For the flow experiments, it was also studied how the platelets adapted their contraction to the external shear stress. Here, not only the actual exerted force was considered but also the direction of contraction. The direction of contraction was defined as the preferred axis of contraction. This corresponded, as already discussed above, to the orientation of the major dipole axis. Thus, the angle between the major dipole axis and the direction of flow was determined.

To determine the direction of flow, classically, tracer particles such as fluorescent beads are used. However, this was not feasible in this case. Beads showed a tendency to stick to the gel or already attached platelets, disturbing the measurements. As we wanted to observe the flow at the position where the platelets attached and also simultaneously to the attachment, another method was employed. Additional cells were always delivered with the buffer flow but not necessarily attaching to the gel at the observed position or even before that. Thus, a constant set of traces of platelets passing by were seen in the BF images. This traces were used to determine the direction of the flow.

From the entire recording, a spot was selected where no cell attached or the flow was disturbed by cell clusters. This area was chosen as large as possible to increase the probability of passing platelets. The underlying beads in the gel were used to calculate the drift in the FOV as previously done (see Section 6.1). Next, in each image, a trace of a by-passing cell was marked if one was detected. Note that as seen in Fig. 6.7 A, the trace had a given width, resulting in variations when tracing them by hand. Automatic tracing was however not possible due to the background noise and the halos surrounding the traces. By measuring the width and length of representative traces, the error in angle determination in both 

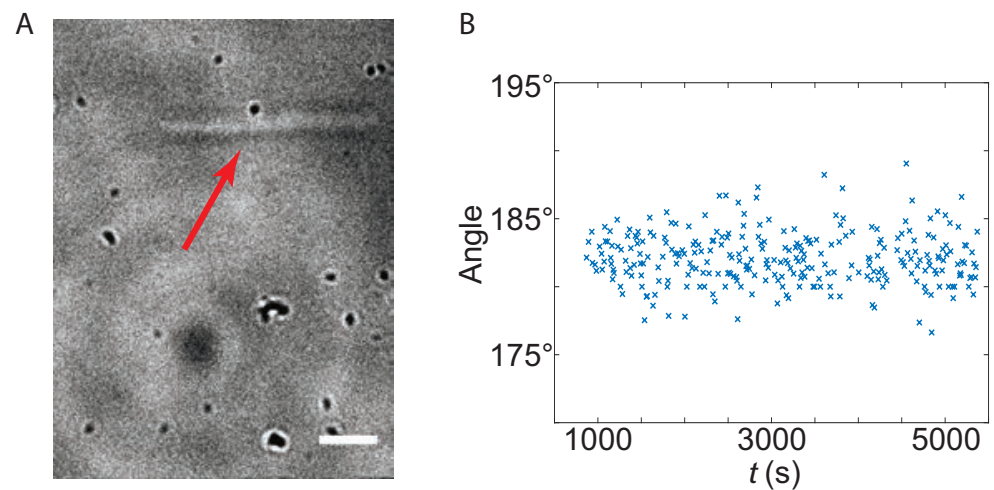

Figure 6.7.: A An example of a bright-field image containing a cell trace (red arrow). $\boldsymbol{B}$ The calculated flow angle for an entire recording. $0^{\circ}$ is defined along the lower image edge, pointing towards the right hand side. Both images were taken at a flow rate of $300 \mu \mathrm{L} / \mathrm{h}$. Scale bar: $5 \mu \mathrm{m}$. The image is taken from Hanke et al. [37].

directions was estimated to not exceed $5 \%$. This analysis was conducted for the entire movie (compare Fig. 6.7 B) and all calculated angles averaged. The averaging was justified by the fact that changes in the flow direction were only possible as a result from air bubbles or other obstacles in the chamber and those data sets were not considered during analysis.

Next, the angle between the major dipole axis and the direction of flow was determined. The dipole axis was calculated as described above in detail. For the following part, the reader is referred to Fig. 6.8 for the visualisation of the reasoning. The angle $\phi$ between the flow and the dipole axis was defined as shown in panel A. Due to the nature of the dipole axis, the angles $\phi$ and $\phi+180^{\circ}$ are identical (panel B), describing the same eigenvector with opposite sign. Furthermore, consider the arbitrary angle shown in panel $\mathbf{A}$, if we assume that we observe the system from above the platelet and the gel. Due to the wide chamber structure, the flow profile perpendicular to the flow, so from one vertical wall to the other, was constant over the gel. This implies that angles of $\phi$ and $180^{\circ}-\phi$ (panel C) as well as $360^{\circ}-\phi$ (panel D) also are considered identical. The only difference towards the original orientation is which 'end' of the platelet points towards the inlet. Thus, all calculated angles were found between $0^{\circ}$ and $90^{\circ}$.

Note that there exists one case where this way of analysis yields an inconclusive result. This is the case of isotropy. To illustrate, let us assume that the axis of the dipole moments remain constant over time. Here, we now have to find the axis 
A

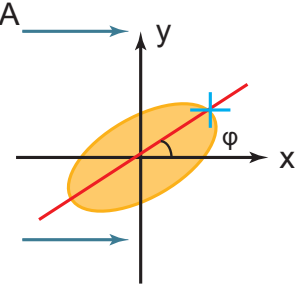

II

C

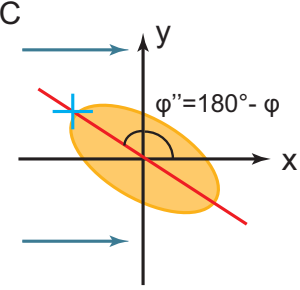

B

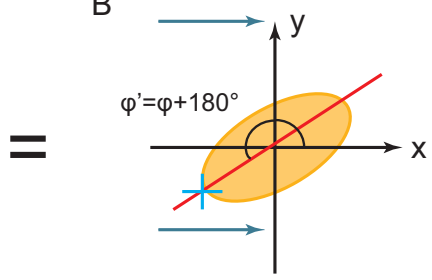

II

$\mathrm{D}$

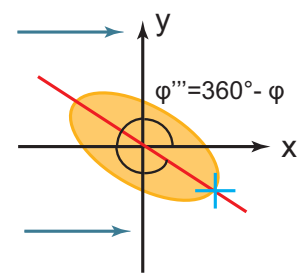

Figure 6.8.: Angles between flow direction and major dipole axis that are considered the same. The platelet is here symbolised as an orange ellipse, the red line denoting the major dipole axis. Here, the flow (blue arrows) goes along the positive $x$-axis. The light blue cross marks the position of the determined dipole vector pointing inwards towards the centre of the cell. The iamge is adapted from Ref. [37].

with larger magnitude. At isotropy, this decision can either not be made at all or is governed by noise. Consequently, the axis of larger magnitude may change between each time point. This results in jumps in the angle $\phi$ of $90^{\circ}$ from one frame to the other. During analysis, this was considered and taken into account if such a case was discovered. 


\section{Results}

In this chapter, the results are presented. First, in Section 7.1, the results of the different algorithms, PIV and optical flow, are compared to each other to validate the applicability of the algorithm of choice. Next, using the chosen algorithm, the observation during first the static experiments (Section 7.2) followed of the flow experiments (Section 7.3), are described. Here, the traction force spatial distribution, the temporal development of the total force and the force dipoles are considered. In particular, possible cues to an adaptation of the contractile behaviour of the platelets in dependency of the external conditions are studied. For the flow experiments, the specially designed flow chamber is characterised both mathematically and experimentally.

Note that Sections 7.1 and 7.2 are part of Hanke et al. [36] and Section 7.3 is part of Ref. [37].

\subsection{Comparison PIV and Optical Flow Algorithms}

Let us briefly recapitulate the two analysis versions we derived in Chapter 5. On one hand, we designed a new version of the commonly used PIV algorithm that accounted for the small size of the platelets as well as the high and dynamic forces exerted by them. This was achieved by including a technique for comparing successive images at time points $t_{i}$ and $t_{i+1}$. To re-calculate the displacement fields compared to the reference frame at $t_{0}$, we introduced the concept of Lagrangian markers. Subsequently, we demonstrated that for this algorithm, no regularisation during FTTC was necessary. Hence we employed an unregularised FTTC to determine the forces. Alternatively, an optical flow method was used. As with the previous approach, a comparison of successive images was applied to account for the force dynamics. Here, the re-calculation of the displacement fields was straight forward due to the moving set of sub- windows. However, for the FTTC part, regularisation was of essence. The reason for the need of regularisation in the latter 
algorithm as opposed to the former one is the level of noise present in the displacement data. In the PIV version, we interpolate the velocity field before tracking the Lagrangian markers, using cubic splines. This reduces the local noise level such that the actual displacements are several orders of magnitudes larger than the noise. In this sense, the interpolation has a slightly smoothing effect. As this interpolation step is missing in the optical flow method, the inherent noise from the image data and the analysis process remains. Hence, regularisation cannot be avoided.

Before starting the actual analysis process, the two different algorithms, namely PIV and optical flow, were compared to guarantee that the Lagrangian marker tracking version of the PIV works as expected. Both the PIV algorithm as well as the optical flow algorithm have previously been shown to work well for larger cells. Here, we studied very small cells exerting comparatively high forces and thus both algorithms had to be adapted to our specific situation. The optical flow approach includes only small changes compared to previously reported versions [45] and was thus taken as a calibration method for the PIV algorithm. This does not mean that the optical flow algorithm was taken as ground truth but instead both algorithms were calibrated to each other by studying the differences in their results. Here, we postulated that the results from both algorithms have to be equal with respect to the computed traction forces since the result needs to be independent of the analysis algorithm used to obtain them.

In Chapter 5, we derived the adaptations we had to implement to be able to analyse the recorded data sets taken over half an hour. While from a mathematical point of view the analysis algorithms were sound, they now were tested on the real data. Here, we intended to calibrate both algorithms to each other and demonstrate their practical applicability to our data. Hence, we first tested whether the algorithms failed to perform for any recorded platelet as previously observed when directly comparing images at time point $t_{i}$ with the reference frame at $t_{0}$. Taking this conventional version of both algorithms, within a few minutes, no reliable velocity field could be determined anymore. Thus, taking different data sets, it was tested if both algorithms yielded a result until the end of recording and if the forces calculated coincided between both approaches. An example of such a data set on a substrate of $19 \mathrm{kPa}$ stiffness is found in Fig. 7.1. Each of the traction force magnitude maps shows the last time point of recording. The white contour denotes the outline of the cell while the black arrows show the direction of the traction force vector. We first observed that the spatial distribution of the traction forces was not identical between both approaches although the location of the force maxima 
remained the same as well as the direction of contraction. For the PIV algorithm, the traction forces were more confined in space while reaching higher magnitudes than the optical flow method. However, taking a look at the total force over time as seen in the lower panels, calculated according to Eq. (5.40), the differences levelled out during integration. We were thus able to conclude that the 'force information' contained within the traction force maps were similar for both algorithms, only differing in their spatial distribution.

Seeing as both algorithms did perform similarly, one analysis programme had to be chosen over the other. Given that the PIV algorithm was specifically designed for the studied cells here, including several mathematical alternative methods compared to standard procedures such as symplectic numerical methods, this approach was taken. Hence, all following results were obtained using the PIV algorithm.

A

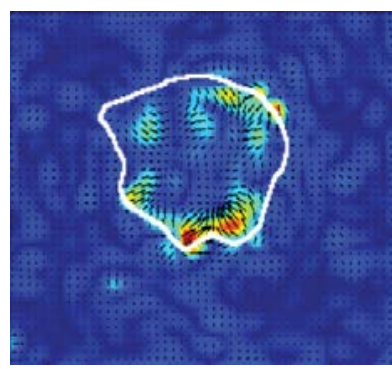

C

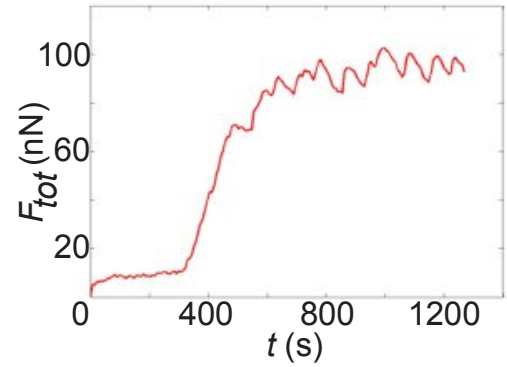

B

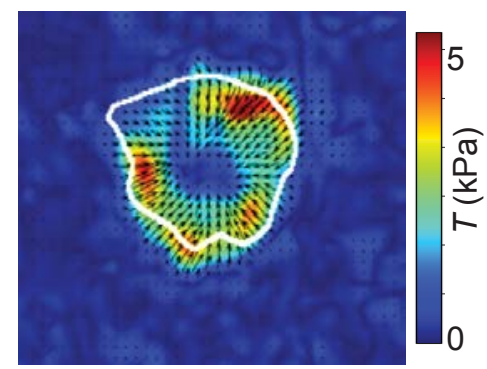

D

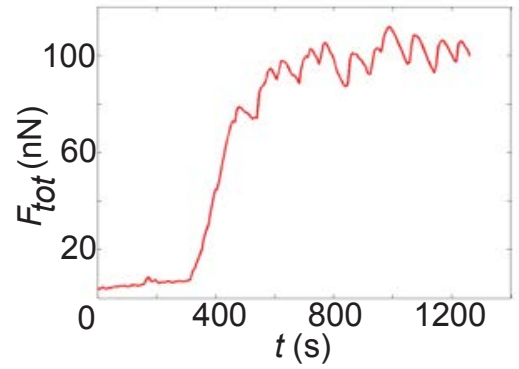

Figure 7.1.: Panels $\boldsymbol{A}$ and $\boldsymbol{B}$ show the traction force magnitude distribution for the PIV and optical flow algorithms, respectively. The white curve denotes the cell outline at the given time point. The black arrows point in the direction of the traction forces. In panels $\boldsymbol{C}$ and $\boldsymbol{D}$, the corresponding total force graphs are depicted. This figure is taken from Hanke et al. [36]. 


\subsection{Contractile Behaviour on Substrata of Different Stiffness}

\subsubsection{Traction Force Distribution in Relation to the Cell's Spread Area}

Analysing the bead movies as previously described in Chapter 5, the distribution of the traction forces was studied at each given time point. This enabled us to investigate the distribution of the traction forces and their location in comparison to the spread cell. Four examples of the cell outline of a fully spread platelet on the corresponding traction force map can be found in Fig. 7.2. As can be seen, the forces exerted by the cell were always found either on or close to the periphery of the platelet. None of the platelets contracted around its centre.
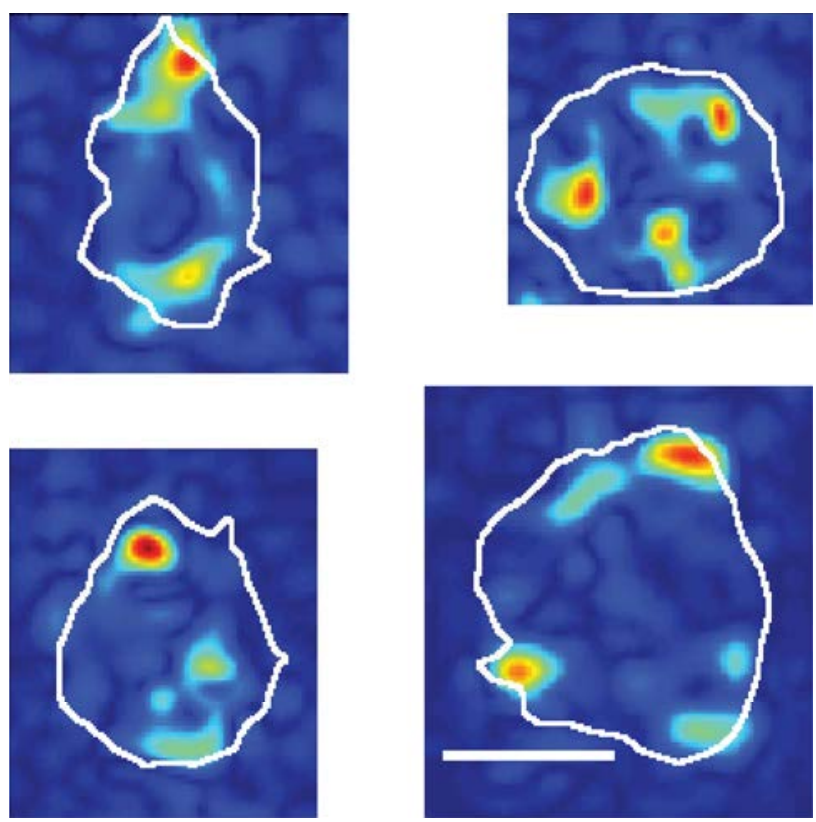

Figure 7.2.: Four examples of the traction force distribution pattern compared to the spreading area of the platelet. The cell's outline is shown as the white curve in each image. Scale bar: $5 \mu \mathrm{m}$. Some of the traction force maps are part of Ref. [36].

At the same time, the contraction was not uniformly distributed around the cell outline but instead exhibited a small number of spatially separated maxima. These maxima varied in number but generally showed two to three, sometimes also four, 
hot spots. Between the maxima, the traction forces were reduced dramatically to a force level close to 0 . In rarer cases, like the platelet shown in Fig. 7.1, the single spots could also nearly span the entire periphery but a completely closed pattern was never observed.

\subsubsection{Temporal Evolution of the Contraction}

Next, let us take a look at the evolution of the total force over time. Before going more into detail of the actual force levels exerted by the platelets on their surrounding, let us take a look at the general temporal behaviour of the cells.

In Fig. 7.3 A, three examples of different contractile behaviours are shown. All cells here were recorded on substrates of stiffness $54 \mathrm{kPa}$. In general, we distinguished between three different behaviours. The first group of platelets, seen in cyan, contracted towards a force plateau. The second group relaxed again after an initial contraction period as shown in green. The last group exhibited a rather dynamic contractile behaviour in that they started to oscillate after an initial increase in force (magenta). These behaviours were found on substrates of all stiffnesses and thus not linked to the elasticity of the surrounding material. Furthermore, combinations of the different behaviours did occur. Note that due to the finite recording time, it was not possible to exclude that some cells showing either a force plateau or oscillations would not relax later. On the contrary, one can safely assume that all cells relaxed again after a sufficiently long time interval when all internal energy storage was used up. Interestingly, the contractile behaviour was not reflected in the dynamics of the spread area, also depicted in Figure 7.3 A. Another example of an oscillating platelet is also shown in Fig. 7.3 B, here recorded on $19 \mathrm{kPa}$. Independent on the contractile behaviour, the platelet spread towards a final area and stabilised. This does not mean that no changes in the membrane were observed but that, given the resolution of our set-up, no quantifiable area fluctuations were seen. Additionally, as can be already estimated from these examples, the area stabilised faster than the total force. The area reached its maximal area within the first 3 to 5 minutes while the force lacked behind some minutes. However, the initial contraction was concluded within the first $10 \mathrm{~min}$ after adhesion. 


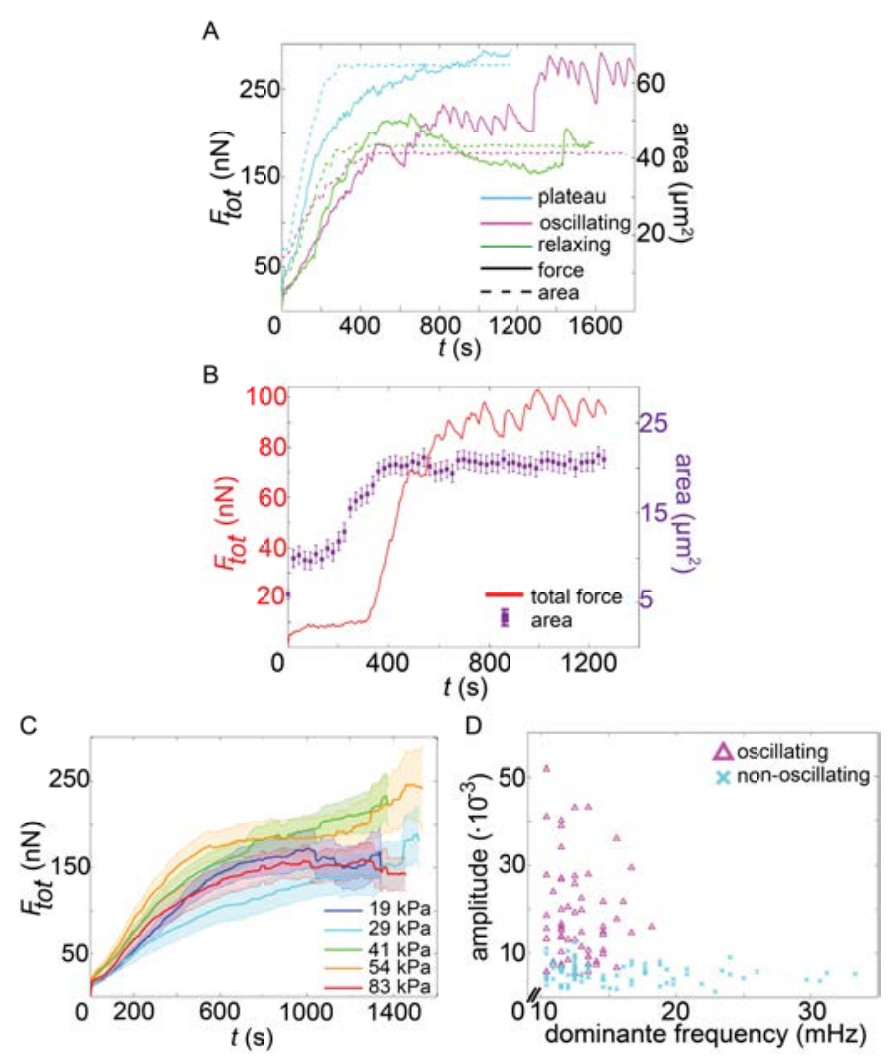

Figure 7.3.: A Three examples of the general temporal contraction behaviour over time. All three cells were recorded on a substrate of $54 \mathrm{kPa}$. In cyan, a platelet is shown that reaches a force plateau. In green, a cell relaxes again after initial contraction. In magenta, a more dynamic platelet is displayed that starts to oscillate after the first contraction. For all three cells, their area development is added in the dashed lines. Here, every third cell image was taken for analysis. $\boldsymbol{B}$ Another example for the spread area with error vs total force development, this platelet recorded on a $19 \mathrm{kPa}$ substrate. As can be observed, the platelets spread faster towards its final area than the force completes the initial contraction period. Also, no synchronisation between the oscillations in area and the force was detected given the resolution and contrast. $\boldsymbol{C}$ The averaged total force curves according to the substrate stiffness. The bold lines denote the averaged force until the time point at which half of the included cells do no longer contribute to the mean. The transparent areas in the same colour show the corresponding standard error. The stiffness varies from $19 \mathrm{kPa}$ in deep blue to $83 \mathrm{kPa}$ in red. $\boldsymbol{D}$ The dominant frequency of all data determined by Fourier analysis. Only the part after the initial contraction was considered. Furthermore, the Fourier analysis was made over the relative changes in force by dividing the analysable force by their mean value. To exclude too low or high frequencies, a Butterworth band-pass filter was applied to only allow frequencies between $10 \mathrm{mHz}$ and $35 \mathrm{mHz}$. Panels $\boldsymbol{A}, \boldsymbol{C}$ and $\boldsymbol{D}$ are taken from Ref. [36]. 
Determining whether a platelet was contracting in an oscillating fashion proved to be not as straight forward as one would expect. First, the total force curves were inspected if a continuous increasing and decreasing behaviour after initial contraction could be observed. If such a behaviour was visible, the underlying bead movies were inspected closely. If a clear in-and-out movement of the beads were seen, indicating a continuous contraction and relaxation of the platelets, the cells were deemed oscillatory. In total, $39 \%$ of all studied platelets under static conditions were thus classified as oscillating. Here, we only distinguished between oscillating and non-oscillating cells. Dividing the cells into all three categories, the percentages were $32 \%, 18 \%$ and $49 \%$ for oscillating, relaxing and plateauing platelets, respectively. The missing percentage was due to rounding. Note that for this division, double-counting of single platelets was possible if a combination of two behaviours was observed.

One could argue that a Fourier analysis would give a clearer criterion on the question whether a cell was oscillating or not. Indeed, this was also tested. Here, the initial contraction was disregarded for the analysis as we were only interested in the behaviour afterwards. The force data at the later time points was then analysed as described in Section 6.2.2. Note that we studied the oscillatory frequency of the relative force to ensure that the magnitude of the mean force in this interval did not influence the result. As an example, $10 \mathrm{nN}$ variation on a cell that contracted up to $40 \mathrm{nN}$ was then deemed to be oscillating while $10 \mathrm{nN}$ variation on a $200 \mathrm{nN}$ contracting platelet most likely was not considered as such. All platelets underwent this analysis and the result can be seen in Fig. $7.3 \mathbf{D}$, where the colour of each marker denotes the classification made previously by eye as described before. We clearly see that non-oscillating cells tended to have lower amplitudes in Fourier space as well as reached higher frequencies, a clear distinction from the oscillating cells was, however, not possible. Both groups overlapped in a band of amplitudes around $7 \cdot 10^{-3}$. While this analysis points towards the correct classification of the eye subdivision, an unique assignment to one behaviour using a Fourier analysis approach was not possible. On the other hand, this analysis allowed us to determine the average oscillatory frequency of the platelets which amounted to $13.5 \mathrm{mHz}$. 


\subsubsection{Dependency of the Force on the Substrate Stiffness}

Having now studied the behaviour of single platelets over time, let us take a look at the average force over time in relation to the underlying substrate. As mentioned previously, we intended to determine the influence of the stiffness of the surrounding on the force development over time of the platelets. Studies on other cell types such as endothelial cells, fibroblasts as well as numerous cancerous cells $[12,35,55,69]$ showed that an adaptation to the external elasticity occurs in those cells. Especially, it was demonstrated that they exert higher forces the stiffer the surrounding is. Is this also the case for the much thinner and smaller platelets? Here, for each substrate stiffness, on average, 32 platelets were analysed, sampled from 5 independent experimental days, as described in the previous chapter, including the given error bound. Following, all force data were averaged per stiffness to gain insight to the general behaviour of platelets in this particular setting. Here, all total force graphs were shifted in time such that the time point of attachment of each cell was set to $t=0$, irrespective of the actual time point in the recording. Keep in mind, that all force data graphs had different length' as the time point $t_{0}$ was chosen individually for each cell. This suggests that the longer the time interval over which was averaged, fewer and fewer platelets were included. To still be able to reach a reliable mean force curve, averaging was stopped at such a time at which half of the platelets stopped contributing to the force. The result can be seen in Fig. 7.3 C in the bold lines. The softest substrate is given in dark blue, the stiffest one in red. Additionally, the standard error for each mean force graph is given as a transparent area in the same colour as the corresponding bold line. Note that we do not show the standard deviation of the averaged force curves. The standard deviation was considerably larger than the standard error such that a clear graphical representation proved difficult. As the standard error, however, is directly related to the standard deviation, we chose to show the error instead.

It was observed from Fig. 7.3 $\mathbf{C}$ that the force does not depend on the stiffness of the environment. Instead of showing lower force values for the $19 \mathrm{kPa}$ curve than for the $83 \mathrm{kPa}$ curve, they both reach about the same force level. Including the large variations between the single platelets in the analysis, no distinction between the different force developments can be made. In other words, given an arbitrary force graph, it would be impossible to relate the platelet to a certain tissue stiffness within the range we studied. The adaptation process observed for other cells did not seem to exist for the given conditions. 
Still, given the large variations noted in Fig. 7.3 C, there must exist a difference between different platelets concerning the force development. If the force exerted by the platelet is not governed by the stiffness of the substrate, what other quantity does influence the contraction?

\subsubsection{Dependency of the Maximum Force on the Platelet Size}

To answer the above question, the final spread area of each platelet determined as described in Section 6.2.1 was studied. As the platelets are produced by a budding-off process from the megakaryocytes, we expected a variation in size to be seen. Indeed, taking a look at the distribution of the final spread area of each cell as seen in Fig. 7.4 A, the size varied between $17 \mu \mathrm{m}^{2}$ to nearly $100 \mu \mathrm{m}^{2}$. On average, the analysed platelets exhibited a size of about $40 \mu \mathrm{m}^{2}$.

Next, the maximal force each platelet exerted on the substrate within the $30 \mathrm{~min}$ of recording time was determined. This was generally not the last time point of recording but was often reached beforehand, especially for relaxing cells. Plotting the maximal exerted total force of each platelet $v s$. the final spread area resulted in the scatter plot shown in Fig. 7.4 A. Again, as previously noted concerning the total force development with regard to the gel elasticity, no difference was seen between the different substrate stiffnesses. At the same time, a clear increase in maximum force was seen with growing area. However, the growing variance in force with larger area, made a linear fit as done in Refs. [12,35] infeasible.

Instead of a linear fit, the model introduced in Section 3.5 (compare also [36]) was used. Here, we modelled the platelet as a thin, circular disc coupled to an elastic substrate by a set of springs (compare Fig. 7.4 B). Briefly, the platelet was characterised by its elastic modulus $E_{c}$, its Poisson's ratio $\nu_{c}$, its height $h_{c}$ and radius $r_{c}$. We further defined the substrate stiffness as $E_{s}$, its Poisson ratio $\nu_{s}$ and its height $h_{s}$. The adhesion layer between both elastic layers was characterised by the adhesion layer stiffness density $\frac{N_{a} k_{a}}{L}$, where $N_{a}$ denotes the number of anchor proteins, $k_{a}$ their stiffness and $L$ the platelet size. The combined stiffness density of the platelet and the substrate is given by $Y$. The contraction was lastly characterised by two quantities, the localisation length $l_{L}$ for the traction force decay in space and $\sigma_{0}$ as the active contractile stress of the cell. Given the previously described model using the here defined physical quantities, an expression for the theoretical forces was derived, compare Eq. (3.41). We fitted the latter equation to the maximal force $v s$ area data given the estimates for the different parameters 


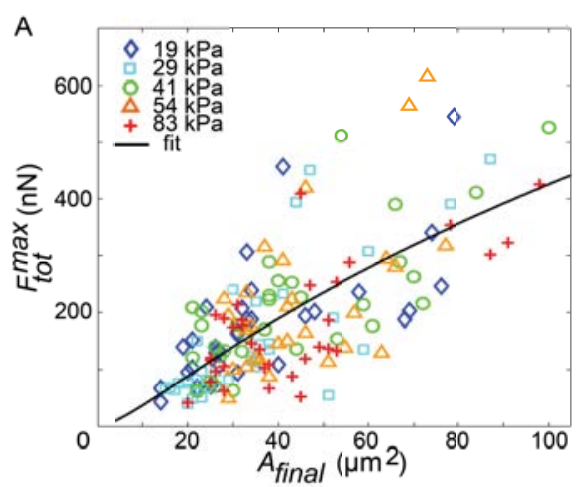

B
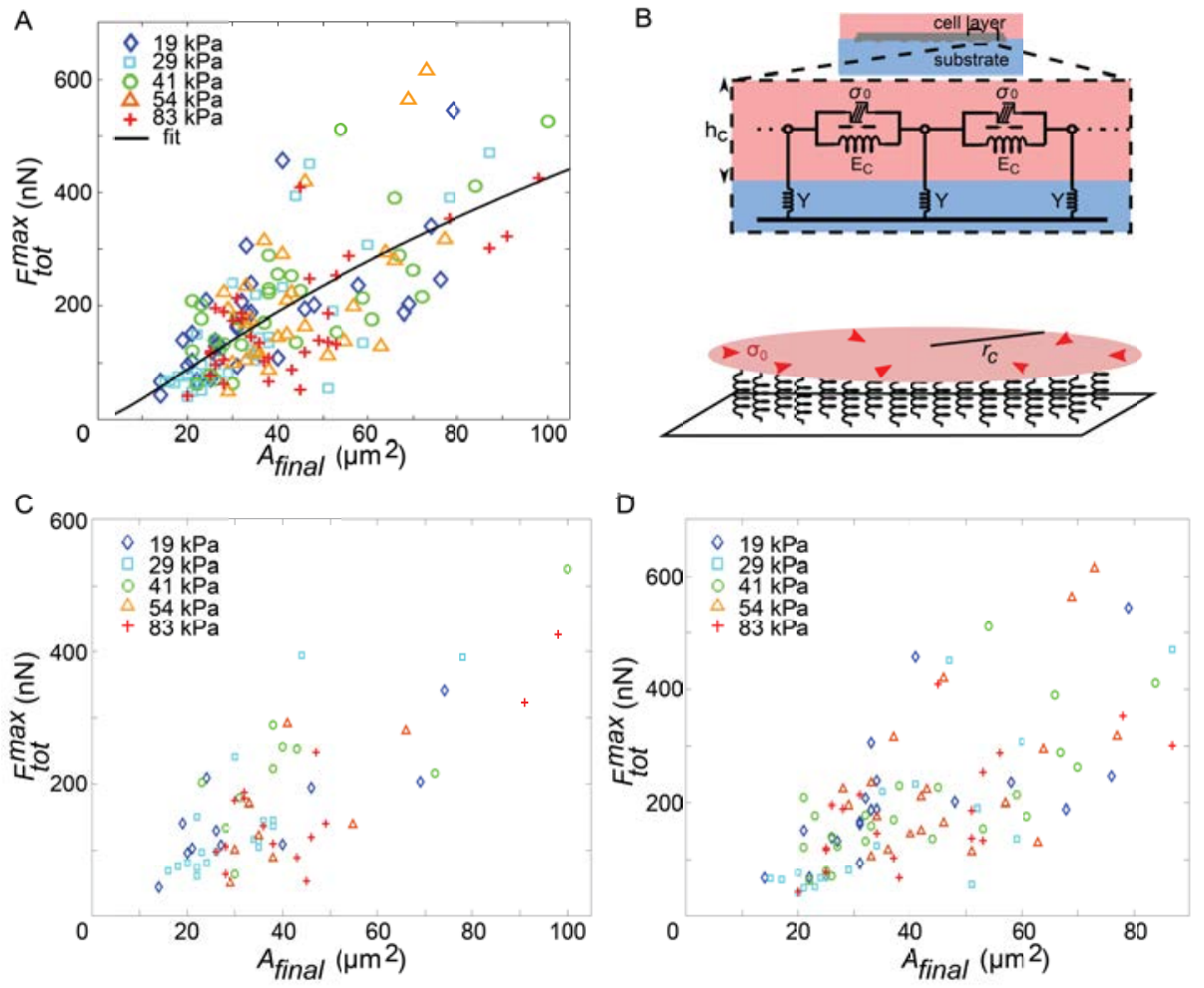

Figure 7.4.: $\boldsymbol{A}$ The maximal reached force within $30 \mathrm{~min}$ of recording vs. the final spread area. A general increase in force is seen with increasing area of the spread platelet. $\boldsymbol{B}$ A sketch of the two-dimensional model used to fit the data shown in $\boldsymbol{A}$. In panels $\boldsymbol{C}$ and $\boldsymbol{D}$, the graph depicted in panel $\boldsymbol{A}$ is separated into oscillating and non-oscillating, respectively. As can be seen, no difference between the groups could be observed. The figure is partially taken from [36].

found in Table 7.1. To find the best fit, the sum of residuals was reduced by finding the best combination of the free fitting parameters $\sigma_{0}$ and $l_{L}$. The best estimate is included in Fig. 7.4 A. ${ }^{1}$ For the fitting parameters, the best estimates were found to be $\sigma_{0}=162 \mathrm{kPa}$ and $l_{L}=1.8 \mu \mathrm{m}$. We included all data here, not separating between oscillating and non-oscillating platelets as both groups did not differ from each other to warrant such a separation, compare Fig. 7.4 C and D.

\footnotetext{
${ }^{1}$ The model fitting was done by D. Probst, University of Heidelberg.
} 


\begin{tabular}{|l|c|c|}
\hline Physical Quantity & Parameter & Value \\
\hline \hline Platelet Young's modulus [58] & $E_{c}$ & $5 \mathrm{kPa}$ \\
\hline Platelet Poisson ratio [121] & $\nu_{c}$ & 0.3 \\
\hline Platelet height [2] & $h_{c}$ & $100 \mathrm{~nm}$ \\
\hline Platelet size & $L$ & $10 \mu \mathrm{m}$ \\
\hline Adhesion layer stiffness density & $\frac{N_{a} k_{a}}{L^{2}}$ & $0.3 \mathrm{nN} / \mu \mathrm{m}^{3}$ \\
\hline Substrate Young's modulus & $E_{s}$ & $19-83 \mathrm{kPa}$ \\
\hline Substrate height & $h_{s}$ & $50 \mu \mathrm{m}$ \\
\hline Substrate Poisson's ratio [64] & $\nu_{s}$ & 0.3 \\
\hline
\end{tabular}

Table 7.1.: Values used for the model fitting described in Section 3.5 and depicted in Fig. 7.4 A.

During the model fitting, we defined two free parameters, namely the localisation length $l_{L}$ and the active contractile stress $\sigma_{0}$. The former of these can also directly be estimated from the traction force maps for each platelet. As mentioned previously, the localisation length denotes a measure on how fast an applied force on the substrate decays in space. Hence, we studied the traction force profiles in space to extract an approximation of the physical value for $l_{L}$. We already touched on the fact that a large variation between platelets was observed in terms of force and area. To compare the physical $l_{L}$ to the estimated value, we thus chose platelets close to the fitted graph. An example of such cell is depicted in Fig. 7.1 with a final spread area of $21 \mu \mathrm{m}^{2}$ and a maximal force of $103 \mathrm{nN}$. The value for $l_{L}$ was determined by first defining the start point of the traction force profiles in the centre of the cell and secondly, automatically finding the force hot spots within the traction force map. For this particular platelet, the lines at which the traction force profiles were considered are shown in the inset of Fig. $7.5 \mathbf{A}$. The traction force along each line was calculated and normalised by its maximal force. All normalised traction forces were then averaged and fitted to the function

$$
\frac{T(r)}{T_{\max }}=\frac{I_{1}\left(\frac{r}{l_{L}}\right)}{I_{1}\left(\frac{r_{c}}{l_{L}}\right)} .
$$

For the platelet depicted in Fig. $7.5 \mathbf{A}$, a value of $1.3 \mu \mathrm{m}$ was found, in good agreement with the value estimated from the fit to all data. We thus concluded that the estimates from the theoretical model very well describe the actual measurements. In the following, we used the value for $l_{L}$ as derived from the model. 
The reason for this choice was the variation found for the $l_{L}$ values between the platelets close and farther away the fitted graph. As we knew that the graph was a good representation of all data, we continued with this value.

Let us briefly go back to the model fit in Fig.7.4 A. Here, we observe that no point of saturation at larger areas was reached. From Eq. (3.42), assuming that $r_{c} \gg l_{L}$, we derived that a saturation in force was reached such that $F_{\text {theo }}=2 \pi h_{c} r_{c} \sigma_{0}$ described its asymptotic maximum value. Given the set values of the cell and substrate characteristics as well as the estimates for the free fitting parameters, a saturation was calculated to be found at a platelet radius of approximately $30 \mu \mathrm{m}$, corresponding to an area of $2800 \mu \mathrm{m}^{2}$, a size two orders of magnitude larger than the real platelet size. Continuing along this line, we derived in Section 3.5 that the total force depends on cell properties $E_{c} h_{c}$ and on the adhesion layer stiffness density $\frac{N_{a} k_{a}}{L^{2}}$. We previously observed that no influence of the substrate stiffness on the total force was measurable. This was further explored by considering the definition of $l_{L}$ as given in Eq. (3.36) and the relation between the force and the localisation length. For different heights of the cell itself as well as different adhesion layer stiffness densities, the approximations shown in Fig. 7.5 B. were derived. In our specific case of platelets, we know from literature that the actual height was $100 \mathrm{~nm}$ or below. Within this regime, the stiffness density did not influence the result significantly. Most interestingly, we noted that a saturation in the force was detected below a substrate stiffness of $10 \mathrm{kPa}$, suggesting that all stiffnesses higher than $10 \mathrm{kPa}$ result in a maximal force response in the platelet. In other words, given the physical properties of the platelets, especially their size, and our data, we were able to show that platelets are unable to sense a difference in the stiffness range studied here in terms of total force. ${ }^{2}$

Until now, we saw that platelets contracted near their periphery, exhibited three distinct behaviours or a combination of these and did not adapt to the stiffness of their surrounding in the studied range of elasticity. The exerted maximum force, however, was dependent on the size of the cells. Using the model of a contractile circular disc with an elastic coupling to the substrate, it became clear that the size of the platelets played an important role in the elasticity insensitivity. In particular, from our data, we predicted that due to their small height, a saturation in total force would not be detected for reasonable platelet sizes and an insensitivity

\footnotetext{
${ }^{2}$ The calculations depicted in Fig. 7.5 B were conducted by A. Zemel, Hebrew University of Jerusalem.
} 

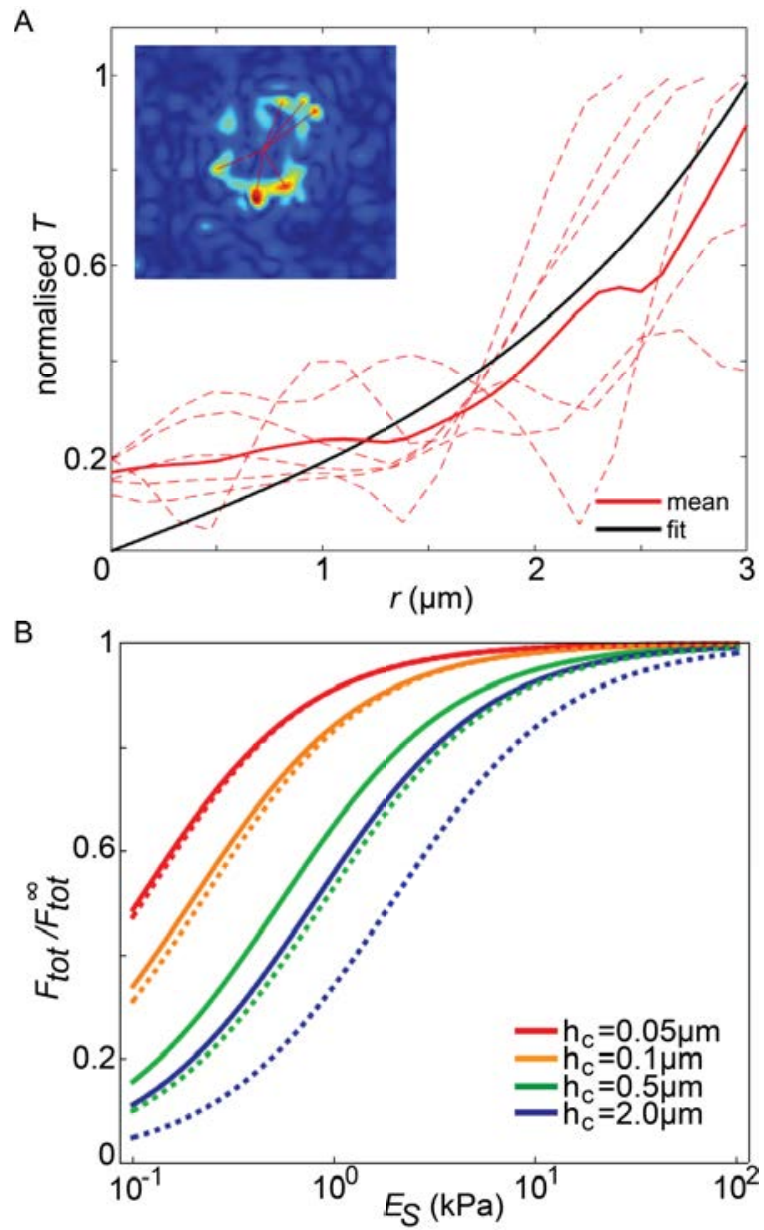

Figure 7.5.: $\boldsymbol{A}$ The localisation length can be determined from the traction force maps as shown here. For the traction force map depicted in the inset, the connection lines between the cell centre and the force maxima are determined (red lines). Along these lines, the relative traction force is calculated (dashed lines) and from the averaged curve (bold), $1_{L}$ is determined via fitting (black) according to Eq. 7.1. For this particular cell, the total force was $103 \mathrm{nN}$, the area $21 \mu \mathrm{m}$ and the localisation length $1.3 \mu \mathrm{m}$. $\boldsymbol{B}$ From the model and the estimation of the localisation length, the dependency of the total force on the substrate stiffness can be estimated. This is depicted here for different cell heights and adhesion layer stiffness density. The bold lines denote a stiffness density of $0.3 \mathrm{nN} / \mu \mathrm{m}^{3}$ and the dashed lines $1 \mathrm{nN} / \mu \mathrm{m}^{3}$. The images are taken from Hanke et al. [36].

exists to substrates of stiffnesses over $10 \mathrm{kPa}$. However, that still does not explain the different behaviours observed. Hence, another physical measure was explored as to explain the different behaviours. 
As we could already see in Fig. 7.4, panel $\mathbf{C}$ and $\mathbf{D}$, neither the final spread area nor the maximal total force was correlated with the individual contractile behaviour of each platelet. Could the behaviour then correspond to an internal structure? As was shown previously in Ref. [25, 48, 90], the actin cytoskeleton may adapt several different shapes such as a pointy ellipse with dense end points or a triangular shape. Let us assume that the major transfer of force from the cytoskeleton to the substrate is done at the pointed ends of the inner actin structure. Then a triangular actin network generally would yield to a more isotropic contraction pattern compared to an elliptical structure. In particular, a triangular actin network does then correlated to, e.g., three distinct hot spots in the traction force distribution as seen above. Can we explain the observed oscillations by a different inner actin architecture? To study this possible aspect, the force dipole was calculated as described in Sections 5.5.1 and 6.2.3. To determine the deviation from the isotropic contraction, the dipole quotient $q$ was determined as given in Section 5.5.2.2 using the error estimation via variance calculation approach.

\subsubsection{Force Dipole Distribution}

For all traction force data sets, the force dipole was determined. One example of the force dipole can be found in Fig. 7.6, another in Fig. 6.6. The dipole was calculated in orientation and magnitude for each given time point apart from those images where no traction force was detected. Hence, a distinguishable dipole magnitude was only observed if an actual force existed (comp. Fig. 6.6 B to the corresponding force curve in Fig. 7.1 C).

Taking a closer look at panel $\mathbf{B}$ in Fig. 6.6, a very synchronised behaviour between the major and minor dipole moments was observed. This behaviour was seen in most cells with the exception of highly polarised cells. Here, the minor dipole moment often remained close to $0 \mathrm{~J}$ or developed partially independently from the major dipole moment as seen in Fig. 7.6 B at about 500 s. Note that always the (absolute) magnitudes in dipole are shown. As the considered traction forces were contractile, the actual eigenvalues were negative.

To determine the degree of isotropy, the dipole quotient $q$ was calculated as described previously in Sections 5.5 and 6.2.3. An example is depicted in Fig. 6.6 C for a synchronised behaviour and in Fig. 7.6 C for an unsynchronised behaviour. Clearly, during the time interval before initial contraction at which the dipole magnitudes were dominated by noise in the images (synchronised case, approximately the first $400 \mathrm{~s}$; unsynchronised case, about $100 \mathrm{~s}$ ), the $q$ changed quickly between 
neighbouring time points. During the interval of distinct dipole moments, this variation decreased, leading to a more stable quotient. Only this time interval was considered during averaging. In the synchronised example, this included all data after initial contraction as indicated in panels $\mathbf{E}$ and $\mathbf{F}$. Contrary, the dipole quotient in Fig. 7.6 C, only showed a smaller time interval of little variance between about $200 \mathrm{~s}$ and $700 \mathrm{~s}$, after which the noise increased due to the relaxing and slightly asynchrony in behaviour of the platelet. Here, accordingly, a shorter interval for averaging was used. Interestingly, the synchronised behaviour of the majority of platelets directly indicates that the force pattern and thus the force transmitting network is established during the initial stages of spreading and does not change upon contraction within the first half an hour.

As we were first interested in whether the dipole ratio varied with the behaviour, we first divided all platelets into two groups, oscillating vs non-oscillating. Subsequently, the dipole quotient $q$ was calculated for each platelet and assigned to its corresponding group. The accumulated averaged quotients $q$ are shown in panel $\mathbf{D}$. We directly saw that, on average, platelets in both groups deviated slightly from isotropy. The median of both groups was 2.2 and 2.3 for the oscillating and nonoscillating platelets, respectively. Due to the non-normal distribution in $q$ in each group, a Wilcoxon rank sum test was applied to test for significant differences between them. No such difference was detected, suggesting that the oscillating behaviour did not coincide with a different cytoskeletal structure.

Additionally to studying the dipole quotient in the context of the temporal contractile behaviour, we also investigated the dependency of $q$ on the substrate stiffness. We previously demonstrated that the total force did not depend on the stiffness due to the small size of the platelet. If the ratio was to change with respect to the substrate stiffness, this would indicate that although the force was not adapted, the force transmitting system was. Calculating the dipole quotient as before, the accumulated data is found in Fig. 7.7 C. At a first glance, the differences were not large between the single stiffnesses, however, a significant difference between the distributions of $19 \mathrm{kPa}$ and $83 \mathrm{kPa}$ as well as $29 \mathrm{kPa}$ and $83 \mathrm{kPa}$ was observed $(p<0.1)$. Thus, an increasing trend in the anisotropy was established, indicating a higher degree of polarisation on stiffer substrates than on softer.

Lastly, let us take a look at the actual values of $q$ that we derived. Independent of the partition of the data we investigated, the median of the distributions was always found between 2 and 3. Pooling all data together, irrespective of the platelet's temporal contractile behaviour or the corresponding substrate stiffness, the median was calculated to be 2.2 and the mean 2.5. We simulated 500 different 


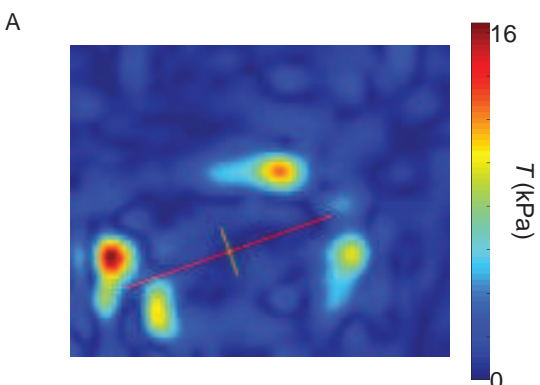

C

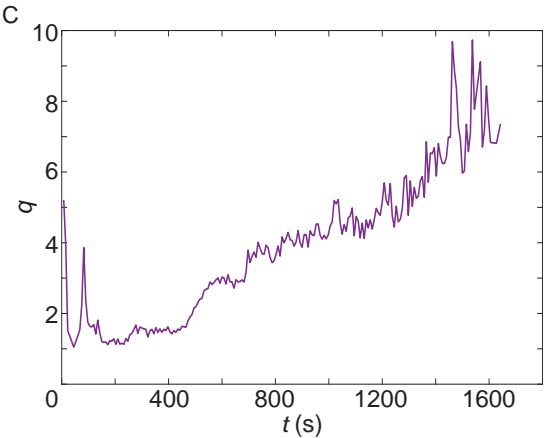

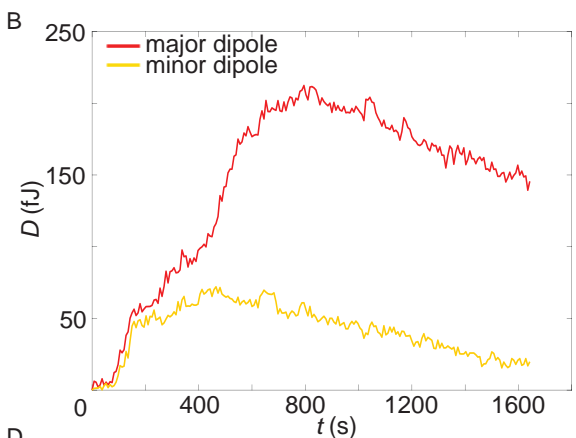

D

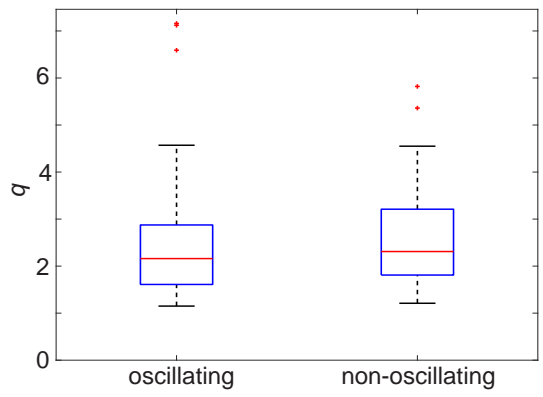

Figure 7.6.: Force dipole of a contracting platelet. A Example of major (red) and minor (yellow) dipole axis on a magnitude traction force map. The length of each axis is scaled to the actual norm of the corresponding vectors. $\boldsymbol{B}$ The temporal evolution of the dipole moments in magnitude. $\boldsymbol{C}$ The dipole quotient $q$ calculated from the dipoles shown in panel B. $\boldsymbol{D}$ Box plot for all average dipole quotients $q$ partitioned into two groups of platelets, oscillating and nonoscillating. The median of the groups are 2.2 and 2.3 , respectively.

point force patterns to gain an insight into what dipole ratio distributions we were to expect of a random distribution. Here, a total of 8 point forces were set around the periphery of a circle, in agreement to the traction force patterns described above. The first seven forces were all of equal magnitude, pointing towards the centre of the circle and randomly allotted. The eighth force was situated in such a way that the vectorial sum of all traction forces was 0 within the simulation. Three examples of such distributions are found in Fig. $7.7 \mathbf{A}^{3}$. For each of the 500 force patterns, the dipole quotient was calculated. The entire distribution in terms of $q$ is found in Fig. 7.7 B, along with the distribution calculated from the measured data. The distributions are in good agreement with each other. The same result is seen in the box plot in Fig. $7.7 \mathbf{C}$, where the simulated ratios exhibited an

\footnotetext{
${ }^{3}$ The simulation was performed by D. Probst, University of Heidelberg.
} 
equivalent median to the distributions on the different substrate stiffnesses. Note that we only included 30 randomly sampled $q$ values, in accordance to the number of platelets included in the other groups depicted here. Hence, we conclude that the slight force anisotropy seen in the platelets is equivalent to a random distribution in force along a circular circumference.
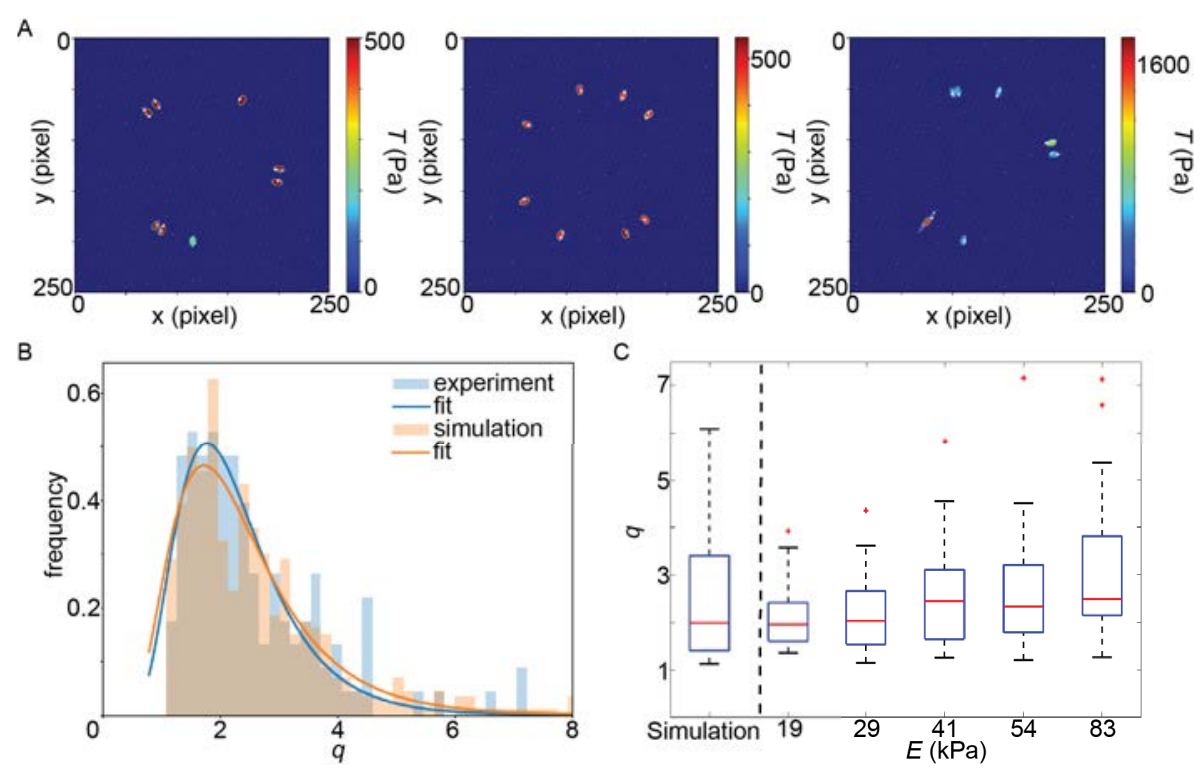

Figure 7.7.: A Three examples of randomised point force patterns containing 8 point forces. All forces are set on the circumference of a circle and their net vector force is $0 . \boldsymbol{B}$ The dipole ratio distribution of the simulated ratios (orange) fit well with the determined distributions from the experiments (blue). $\boldsymbol{C}$ The agreement between the simulation and experiments is also seen when separating the dipole quotients according to the substrate stiffness. For the simulation, out of 500 different force patterns, 30 were randomly sampled for this figure. A slight increasing trend is observed with increasing elasticity of the substrate, where the distributions for $19 \mathrm{kPa}$ and $29 \mathrm{kPa}$ are significantly different from that of $83 \mathrm{kPa}(p<0.1)$. The figure is taken from [36].

To briefly summarise, for the static experiments, we first calibrated both algorithms against each other, demonstrating that both approaches led to the same results in terms of total force. In the following analysis, we always used the PIV algorithm as it was specifically tailored to fit our problem. We showed that platelets contract along their periphery at a distinct number of spots. Platelets could be subdivided into three different categories concerning their temporal contractile behaviour; they either reached a force plateau, relaxed after initial contraction or started to oscillate after contraction with an average frequency of $13.5 \mathrm{mHz}$. 
Combinations between these behaviours were observed. The different contractile developments were not reflected in the spread area evolution. The cells spread rapidly towards a final area, stabilising faster than their corresponding total forces. Additionally, we experimentally demonstrated that the total force of the platelets is independent within the studied stiffness range. This was due to the small size of the platelets as we derived theoretically by modelling the cells as elastic, circular discs coupled to the substrates. Here, we predicted that platelets were only mechano-sensitive in a stiffness range well below $10 \mathrm{kPa}$. An adaptation in the total force was observed in correspondence with the spread area, where larger cells exerted a higher force than their smaller equivalents. Lastly, we showed that the contraction of platelets is slightly anisotropic. The degree of anisotropy did not change with the temporal contractile development but possessed an increasing trend with increasing substrate stiffness, suggesting a higher polarisation within the force transmitting network on stiffer substrates. The observed anisotropy, on average, was reproducible by simulating 8 random point forces along a circle's circumference, indicating a generally random force spot distribution.

\subsection{Blood Platelets Under Flow Conditions}

\subsubsection{Device Characterisation by Simulation}

To start the characterisation of the our microfluidic device, both the measuring chamber as well as the mixing channel were simulated using COMSOL Multiphysics $^{4}$. For the geometry of the combined device, the reader is referred back to Fig. 3.3. The measuring chamber was investigated towards its velocity profile and the expected shear rates above the gel. This shear rate was then taken as the shear rate experienced by the platelet during the experiments and the goal here was to be in a shear rate regime as found in larger human veins. Later on, the experiments were conducted using three different flow rates, namely $300 \mu \mathrm{L} / \mathrm{h}$, $500 \mu \mathrm{L} / \mathrm{h}$ and $700 \mu \mathrm{L} / \mathrm{h}$. Another flow rate, $1000 \mu \mathrm{L} / \mathrm{h}$, was tested concerning the attachment rate but not employed during experiments. In Table 7.2, the analytically calculated mean velocities within the measuring chamber are given for all four tested flow rates. Here, we assumed that the gel was $3 \mathrm{~mm}$ wide and $30 \mu \mathrm{m}$ high while the chamber itself was $4 \mathrm{~mm}$ wide and $110 \mu \mathrm{m}$ high, which corresponds to the average dimensions of both the substrate as well as the chamber. For the same dimensions, the Reynolds number is included in the table.

\footnotetext{
${ }^{4}$ COMSOL AB, Stockholm, Sweden
} 


\begin{tabular}{|c|c|c|}
\hline Flow rate $[\mu \mathrm{L} / \mathrm{h}]$ & Mean velocity $[\mu \mathrm{m} / \mathrm{s}]$ & Reynolds number \\
\hline \hline 300 & 236 & 0.04 \\
\hline 500 & 393 & 0.07 \\
\hline 700 & 550 & 0.09 \\
\hline 1000 & 786 & 0.13 \\
\hline
\end{tabular}

Table 7.2.: The mean velocity and corresponding Reynolds number found within the measuring chamber for the different tested flow rates.

\subsubsection{Simulation of the Velocities and Shear Rates Inside the Measuring Chamber}

To be able to calculate the velocity profile, the Navier-Stokes equation (Eq. (3.17)) was solved using the finite element method. Given the analytically calculated Reynolds numbers, we expected the flow profile to be laminar, i.e. resembling that of a parabola. As the substrate did not span the entire chamber, two different profiles were anticipated, one between the substrate's surface and the chamber ceiling and another between the glass slide and the ceiling. For simplicity, we assumed that the substrate was completely centred inside the chamber's channel. This resulted in the geometry to become symmetric with respect to a vertical plane through the chamber's middle. Hence, only half of the chamber had to be simulated, compare Fig 7.8 A. For all simulations, we assumed that the plateletthrombin-buffer solution, from a fluid dynamics point of view, behaved as water with its corresponding viscosity and density. This was justified by the fact that water was the major component of the liquid. Note that water is a Newtonian fluid. For the simulations, the physical properties of the chamber and substrate are found in Table 7.3. We assumed a rectangular chamber, disregarding the rounded endings of the actual chamber. As these endings were avoided during assembly of the chamber to reduce the chance of the substrate being positioned underneath the PDMS cast, this simplification was warrantable. Overall, the geometry of the ending had no influence on the flow profiles at the measuring site in the middle of the chamber.

Solving the Navier-Stokes equation for our geometry, we did indeed observe a laminar flow regime. For the flow rate of $700 \mu \mathrm{L} / \mathrm{h}$, the flow profile around the border of the substrate is depicted in Fig. 7.8 B. The laminar flow is seen both before the substrate as well as above it. As we already noted in Table 7.2, the velocity scales linearly with the flow rate. Hence, all velocities scale accordingly in the flow profiles and are thus not shown here. From the velocity profiles, the shear rate was directly derived according to Eq. (3.19). For the lowest and highest 
A

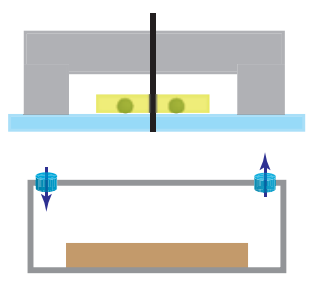

C

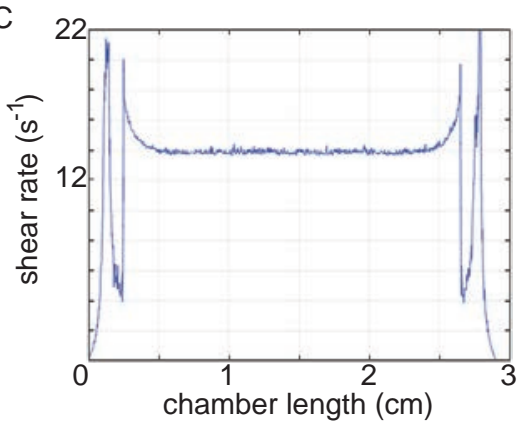

B

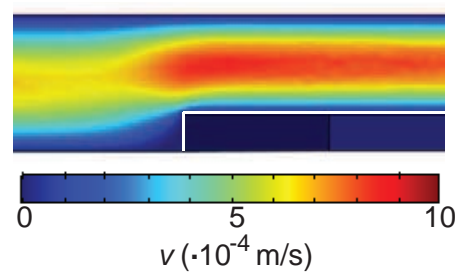

D

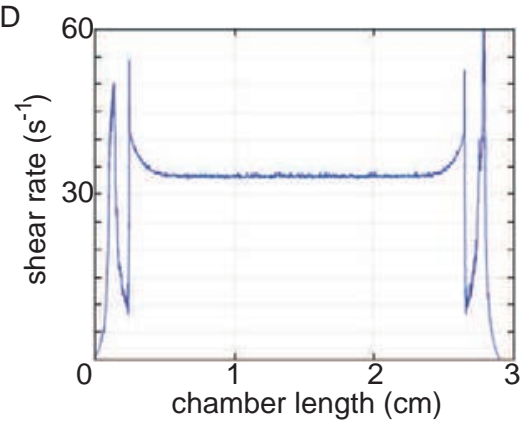

Figure 7.8.: A The geometry used for the theoretical characterisation of the flow chamber. The upper sketch shows the cross section along the width of the channel (gel - yellow with green beads, channel walls - gray and glass slide blue). Note that the problem is symmetric towards a plane in the middle of the channel, denoted here by the black line. In the lower sketch, the cross-section along the length is sketched, denoting the geometry used for the simulations, containing the inlet and outlet (blue with black arrow). $\boldsymbol{B}$ The laminar flow velocity profile at the border of the substrate for a flow rate of $700 \mu \mathrm{L} / \mathrm{h}$. The substrate edge is marked by white lines. The shear rates directly above the gel (height $30 \mu \mathrm{m}$ ) along the centre of the chamber, from inlet to outlet, is shown for $300 \mu \mathrm{L} / \mathrm{h}$ and $700 \mu \mathrm{L} / \mathrm{h}$ in $\boldsymbol{C}$ and $\boldsymbol{D}$, respectively. The high peaks in the graph denote the positions of, from left to right, the inlet, the first gel edge, the second gel edge and the outlet. The linear scaling of the shear rate with the flow rate is illustrated when comparing the values above the substrate for $\boldsymbol{C}$ and $\boldsymbol{D}$. Note that panel $\boldsymbol{C}$ was adapted from Hanke et al. [37].

flow rate utilised during the measurements, the shear rates directly above the gel are shown in Fig. $7.8 \mathbf{C}$ and $\mathbf{D}$, respectively. The shear rate was taken along the length of the entire chamber, from one narrow side to the other. The dimensions from the substrates are noted by the inner high peaks in the graphs, from $2.5 \mathrm{~mm}$ to $26.5 \mathrm{~mm}$. From the simulations, the shear rates are calculated to be approximately between $14 \mathrm{~s}^{-1}$ and $33 \mathrm{~s}^{-1}$ for the flow rates of $300 \mu \mathrm{L} / \mathrm{h}$ and $700 \mu \mathrm{L} / \mathrm{h}$, respectively. Again, the shear rate scales linearly with the flow rate. Previously, the shear rates in larger veins have been reported to start at values of $5 \mathrm{~s}^{-1}$ to $10 \mathrm{~s}^{-1}[91,99]$. Hence, the chamber dimensions and the chosen flow rates allowed 


\begin{tabular}{|c|c|}
\hline Physical Property & Value \\
\hline \hline Chamber height & $110 \mu \mathrm{m}$ \\
\hline Chamber width & $4 \mathrm{~mm}$ \\
\hline Chamber length & $29 \mathrm{~mm}$ \\
\hline Substrate height & $30 \mu \mathrm{m}$ \\
\hline Substrate width & $3 \mathrm{~mm}$ \\
\hline Substrate length & $24 \mu \mathrm{m}$ \\
\hline Inlet radius & $0.19 \mathrm{~mm}$ \\
\hline
\end{tabular}

Table 7.3.: The values used for the simulation of the measuring chamber. Note that the actual geometry size is given while the simulation was conducted on half of the geometry, using symmetrical boundary condition.

us to mimic the flow situation found in human veins. At the same time, the size of the substrate was large enough to guarantee that spots for recording of attached platelets were found.

\subsubsection{Simulation of the Diffusion and Transport Processes Inside the Mixing Channel}

We have now shown that the measuring chamber we designed fulfilled our physical criteria such as the desired flow rate. Its practical application is presented in the following section. Before the actual experiments, the mixing device was also characterised. The mixing channel was introduced into the set-up to satisfy two purposes. On one hand, within the device, the thrombin was mixed with the platelet solution such that the end concentrations were the same as used for the static experiments. On the other hand, by mixing the substances in an external device in lieu of the syringe ensured that the reaction time between them was of the order of minutes instead of hours. Due to the controlled mixing process, all platelets recorded at a given place on the substrate had about the same amount of time to interact with the thrombin, independent at which time point they were recorded. Here, we defined the time from the complete mixing of the substances to the entrance of the measuring chamber as the reaction time. Due to the chosen flow rates and the needed length of tubing to connect both devices, the reaction time was estimated to be under 5 min for all flow rates. In particular, given the inner radius of the connecting tubing as stated in Table 7.3 and length of about $12 \mathrm{~cm}$ needed to link the devices on the microscope stage, the dwell time was estimated to be about $2.7 \mathrm{~min}$ for the slowest flow velocity at $300 \mu \mathrm{L} / \mathrm{h}$. The dwell time in the mixing channel (the dimensions are noted in Table 7.4) after complete mixing 
was in the order of a few seconds. The reaction time created with this set-up correlated with the time difference we observed in the static experiments between the mixing of thrombin and platelets and the first attached cells on the substrate.

\begin{tabular}{|l|c|}
\hline Physical Property & Value \\
\hline \hline Channel height & $180 \mu \mathrm{m}$ \\
\hline Channel width & $200 \mu \mathrm{m}$ \\
\hline Channel length from mixing region & $22.3 \mathrm{~mm}$ \\
\hline Channel length in simulation & $15 \mathrm{~mm}$ \\
\hline Platelet radius & $1.5 \mu \mathrm{m}$ \\
\hline Diffusion coefficient platelets & $1.63 \cdot 10^{-13} \mathrm{~m}^{2} / \mathrm{s}$ \\
\hline Platelet concentration & $3.32 \cdot 10^{-11} \mathrm{~mol} / \mathrm{m}^{3}$ \\
\hline Diffusion coefficient thrombin $[40]$ & $8.7 \cdot 10^{-11} \mathrm{~m}^{2} / \mathrm{s}$ \\
\hline Thrombin concentration & $3 \cdot 10^{-4} \mathrm{~mol} / \mathrm{m}^{3}$ \\
\hline
\end{tabular}

Table 7.4.: The values used for the simulation of the mixing channel. Note that the first three entries are given for the actual device while the simulation was conducted on half of the geometry, using symmetrical boundary condition along the length.

To simulate the mixing process within the mixing channel, we combined the Navier-Stokes equation with the convection-diffusion equation (3.21). All combined flow rates from the side inlets and the central inlet summed up to the flow rates used during the experiments. The thrombin solution injected into the side inlets was set to a concentration of $40 \mathrm{u} / \mathrm{mL}$ while the platelets were added at a concentration of $2 \cdot 10^{7}$ cells $/ \mathrm{mL}$, similar to the static case. Hence, to regain the original mixing concentrations of $4 \mathrm{u} / \mathrm{mL}$ thrombin in the platelets solution, the ratio between the flow rates of the single inlet was set to $1: 18$, i.e. a ration of $35-630-35 \mu \mathrm{L} / \mathrm{h}$ for the highest flow rate used in the experiments. All physical quantities needed for the diffusion process, such as the diffusion coefficients, inlet concentrations and the assumed radius of an un-spread platelet, are found in Table 7.4. The diffusion coefficient for the platelets was estimated according to Eq. (3.24). To calculate the concentration of the thrombin, note that $1 \mathrm{NIH}$ unit corresponds to $0.324 \mu \mathrm{g}$ of active enzyme and the molecular weight is $36 \mathrm{kDa}$.

The results of such a simulation can be found in Fig. 7.9. A sketch of the geometry is found in A. Again, the problem was symmetric along the the central plane in length. As the channel is comparatively long, only a part was simulated as marked in the figure. First, we intended to ensure that the platelets were not exposed to such a high shear stress within the channel as to get activated without the addition of thrombin. The most critical flow rate here was the highest one, 
namely $700 \mu \mathrm{L} / \mathrm{h}$. For the first part of the channel, around the position of the side inlet, the shear rate was studied as depicted in panel $\mathbf{B}$. We observed that the maximal shear rate did not exceed $300 \mathrm{~s}^{-1}$. This is a shear rate expected in smaller veins [77] and well below the shear rates found in healthy arteries by an order of magnitude. We thus concluded that an activation by pure shear stress was unlikely. Thus, we investigated the mixing process. We remarked earlier that we expect the platelets to diffuse from the central line towards the outer wall much slower than the thrombin for the reverse direction due to their difference in size. This was well seen in the simulations, compare panel $\mathbf{C}$. While the thrombin rapidly changes its concentration locally, the platelet solution does not. We thus define the point of complete mixture as the point where the thrombin concentration stabilises along the central line of the channel. As shown in Fig 7.9 D for the highest flow rate, this point is always reached within the first centimetre after the position of the side inlet. Hence, we can conclude that the solutions are always mixed completely inside the channel before entering the connecting tubing.

\subsubsection{Testing for Usability of the Flow Chamber}

After mathematically characterising the measuring chamber to demonstrate the compliance to our desired physical requirements in terms of flow profile and shear rate, the chamber was tested in its practical use. First, keep in mind that the finished PAA substrate completed with the fibrinogen coating was treated with an oxygen plasma to facilitate the covalent binding between the glass and PDMS cast. Consequently, it was tested whether both the hydrogel as well as the fibrinogen coating were able to endure this process. Next, our flow chamber needed to be capable to withstand a constant flow over at least $1.5 \mathrm{~h}$ of measuring time additionally to the time that was needed to fill the entire system with fluid three times: first the flushing with MilliQ water for storage, next the washing before recording with HTBSA-buffer and lastly the filling of the system with the platelet-thrombin-buffer solution directly before measurements were started. Lastly, in Section 7.3.1.1, we determined that a laminar flow profile was to be expected in the flow chamber, corresponding to a velocity profile in height of a parabola. From the flow profiles, other physical quantities such as the shear rates were then derived. Thus, we examined whether the actual flow profile resembled the theoretical profile. 
A

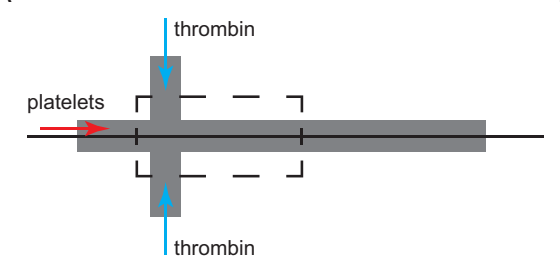

C
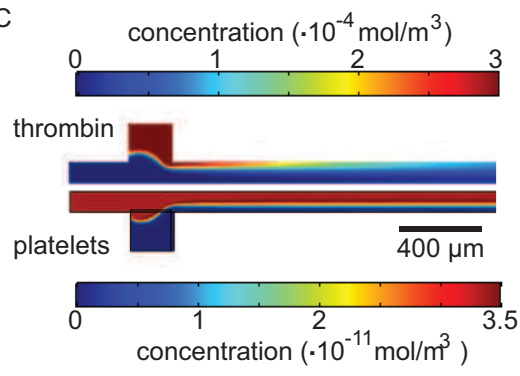

B
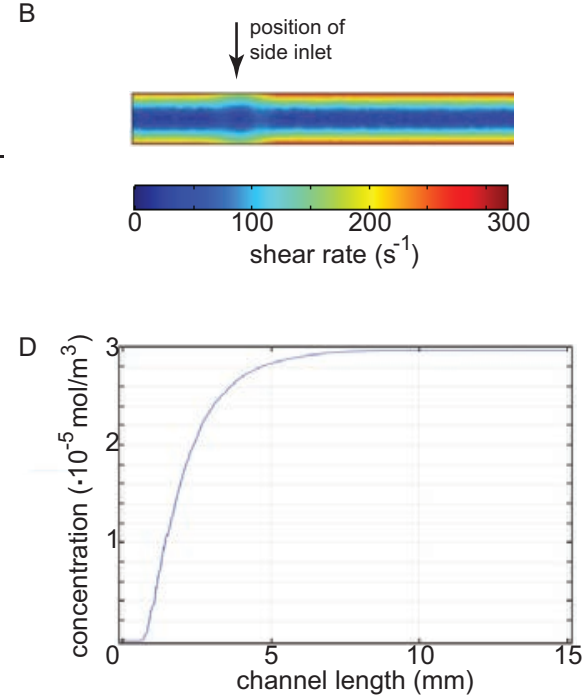

Figure 7.9.: A The general structure of the mixing channel. The thrombin solutions enter from the side inlets, the platelets from the central inlet. We have again a symmetric problem, the symmetry line being along the length of the channel. The geometry is reduced for the simulations to the central part denoted with the dashed rectangle, sufficiently long to see complete mixture from the thrombin. $\boldsymbol{B}$ The shear rate distribution for the highest flow rate of $700 \mu \mathrm{L} / \mathrm{h}$. The shear rate does not exceed $300 \mathrm{~s}^{-1}$. We thus assume no activation due to shear stresses in the mixing channel. $\boldsymbol{C}$ The concentration distribution of thrombin (upper part) and the platelets (lower part) for the first few millimetres. While the thrombin solution diffuses fast, the platelets do not show any noticeable diffusion. $\boldsymbol{D}$ The concentration of thrombin along the central line of the channel. The stabilising concentration marks the even distribution of thrombin within the entire channel. All figures are taken for a flow rate of $700 \mu \mathrm{L} / \mathrm{h}$. The image is adapted from [37]. 
Starting, let us have a look at the fibrinogen coating. From the experiments under static conditions as well as other reported literature (see e.g. [107]), we know that platelets attach to the PAA gels if those are coated with fibrinogen. To ensure that the fibrinogen coating was not destroyed during plasma treatment, the following substrates were prepared for control experiments:

- a PAA substrate with neither coating nor plasma treatment;

- a substrate with fibrinogen coating done after plasma treatment;

- a substrate with fibrinogen coating done before plasma treatment.

For the two latter substrate types, during plasma treatment, a thin PDMS stripe was used to cover and protect the hydrogel as described in Section 4.3.2. Note that the upper part of the chamber, the cast made from PDMS, was not bound to the glass at this stage. As we wanted to check the state of the fibrinogen coating, a labelled fibrinogen was used alongside red fluorescent beads as described in Section 4.2.2.

We first investigated the coverage of the fibrinogen on the substrates. Here, we employed labelled fibrinogen. In the FITC channel, we were able to record a signal, but the intensity distribution within the pictures did show a similar spectrum as substrates without any coating at all. An example for all tested variations is found in Fig. 7.10. Here, A denotes a substrate without protein coating, $\mathbf{B}$ a gel which was first coated with fibrinogen before undergoing plasma treatment and C first underwent plasma treatment before the protein coating. Despite the nonsignificant changes in the intensity between the different substrates, small patterns were observable on gels that had been in contact with fibrinogen, suggesting that some fibrinogen still was attached to the substrate. To ensure that this signal was not originating from single fluorophores attached to the gel, platelets and thrombin were added to the substrate as done during static experiments. While the cells did not spread and indeed were hardly attaching to the gel if no fibrinogen was present, they did spread on substrates that had undergone plasma treatment (compare lowest row in Fig. 7.10). Thus, we concluded that the protein coating was not destroyed during plasma treatment. Furthermore, while spots of uneven surface were found in the bright-field channel, indicating a partial drying during plasma exposure, those were few and easily detectable. During experiments, these were avoided if seen. In the following, due to its easier handling and the reduced stress on the fibrinogen, the approach of using the plasma cleaner after the fibrinogen coating was chosen. 
A
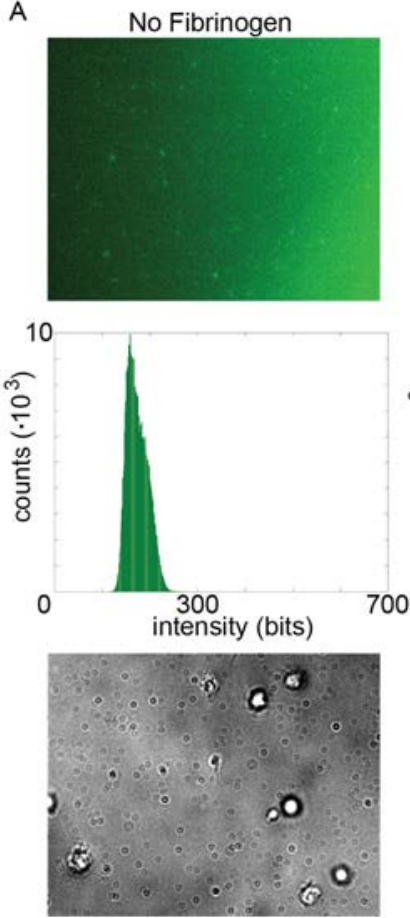

B
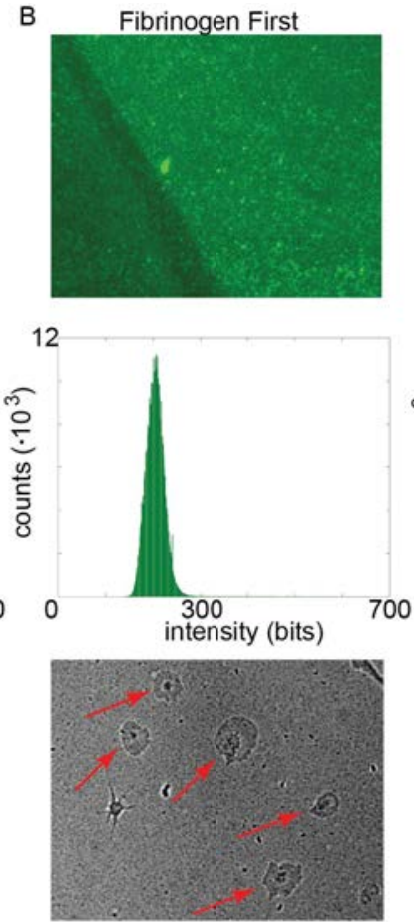

C
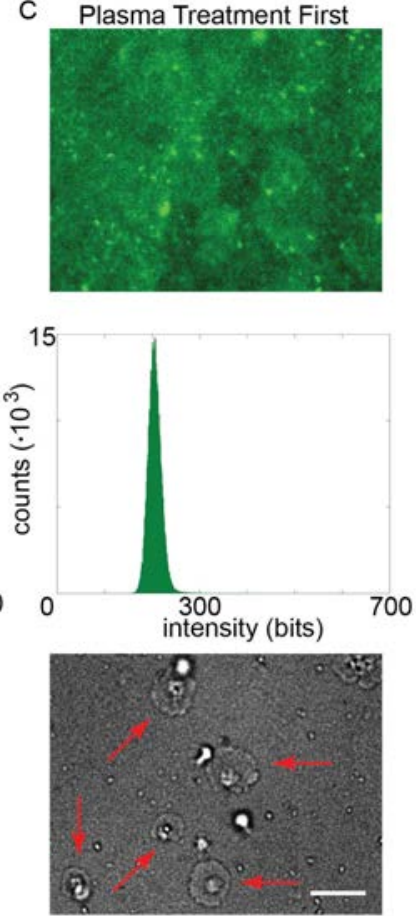

Figure 7.10.: Testing for the coverage of fibrinogen with plasma treatment. The following substrates are shown: a gel with no fibrinogen coating $(\boldsymbol{A})$, a gel first coated with fibrinogen before plasma treatment $(\boldsymbol{B})$ and a gel which was first inserted into the plasma cleaner before the fibrinogen was applied $(\boldsymbol{C})$. The first row shows examples of the fibrinogen coverage, below the corresponding intensity distribution. In the last row, for the same spot, the platelet coverage is shown. Spread platelets are marked with red arrows. The bright white spots with black borders are round cells near the surface but not attached. Scale bar: $10 \mu \mathrm{m}$. 
To reduce the possibility of damage to the substrates during storage, the complete chamber was always stored filled with water as described in Section 4.3.2. The water was injected into the chamber by the inlet tubing using a syringe, also filling the outlet tubing. Both the filling as well as all washing steps were conducted at a higher flow rate of $800 \mu \mathrm{L} / \mathrm{h}$ as described previously. During the filling of the tubing and mixing device with the platelets and thrombin directly before the measurement, a lower flow rate was chosen to avoid unnecessary stress onto the cells or even trigger spreading before reaching the measuring chamber. We observed that devices withstanding both flushing procedures at the higher flow rate and the re-filling at lower velocity did not start to leak during the recording. The tubing at all inlets and outlets on both devices were glued onto the devices to ensure a tight fitting.

Next, to check the flow profiles, the same set-up as for the normal flow experiments was used, including the mixing device. All pumps were driven as during actual experiments with the corresponding flow rates. Instead of platelets and thrombin, the syringes were filled with a solution containing $500 \mathrm{~nm}$ red fluorescent beads in HT-BSA buffer at a mixing ratio of 1:1999. The beads in the solution were employed to visualise the flow inside the flow chamber. They travelled with the liquid and, by illuminating them sufficiently long, described small traces along their pathway. These traces were analysed to determine the velocity of each particle and hence the flow velocity at that point. A spot on the gel was chosen and fixed for all following tests. Two different kinds of tests were conducted, one to determine the horizontal flow profile directly above the substrate, compare the sketch in Fig. 7.11 A, and one to determine the profile vertically to the substrate, see Fig. 7.12 A. Thus, for all three flow rates, both multiple scans directly on the gel surface as well as a scan in height were recorded. Due to the software of the microscope, the exposure time could not be adjusted during the height scan but was kept constant. Consequently, an exposure time was chosen that was sufficiently long to visualise the bead traces directly above the gel while being short enough for faster flowing particles to not leave the FOV during exposure. Here, we used $100 \%$ of the lamp intensity with $200 \mathrm{~ms}$ of exposure time. As a result, these scans were restricted to $20 \mu \mathrm{m}$ above the gel but were always conducted at least twice to increase the sample size within the measured interval. Directly above the substrate, to evaluate the flow laterally to the surface, at least 50 images were taken. The following analysis was carried out by hand using ImageJ. 
We started out by investigating the velocity profile horizontally above the gel, compare Fig. 7.11 A. Along the length of the substrate, no change in velocity did occur for an undisturbed flow, hence, we only considered the velocity profile perpendicular to the flow. To begin with, the recording was divided into three equally sized parts parallel to the flow direction as depicted in Fig 7.11 B. Both the upper part and the lower part of the recording were analysed separately from each other. From the theoretical examinations made previously, we did not expect a difference in velocity between these two sections. If this was proven to be true, we could safely assume in the following that the flow around the platelet at the time point of attachment as well as full spreading was equal on both sides. Otherwise, a closer look at the profile had to be taken. In Fig. 7.11, the estimated velocities above the gel for both $300 \mu \mathrm{L} / \mathrm{h}$ (panel C) and $700 \mu \mathrm{L} / \mathrm{h}$ (panel D) are shown, each corresponding to a recording of 50 single frames with approximately 200 traces in total. Although a noticeable variation did exist between the single traces, the distributions of the velocities between the upper part of the image and the lower part were similar, confirmed by a Wilcoxon rank sum test. This held true for all tested flow rates. We thus concluded that, as expected from theory, the velocity profile horizontally to the gel was constant.

Subsequently, we evaluated the traces recorded during the height scans. The scans were conducted according to the sketch found in Fig. 7.12 A. Before recording, the substrate's height was measured to be $25 \mu \mathrm{m}$. From theory, we did expect a parabolic velocity profile. As we were not able to reliably record the entire profile in height, we added an additional data point at the ceiling of the chamber, namely a velocity of 0 . Note that for this particular measurement, a PDMS cast was utilised which had a total chamber height of $129 \mu \mathrm{m}$, a bit higher than the average chamber. At the same time, the gel was slightly lower than the average substrate used for the simulations in Section 7.3.1. Hence, we anticipated a slightly lower maximal velocity than shown in Fig. 7.8 for the corresponding flow rate. The results for $300 \mu \mathrm{L} / \mathrm{h}$ and $700 \mu \mathrm{L} / \mathrm{h}$ are depicted in Fig. $7.12 \mathbf{B}$ and $\mathbf{C}$, respectively. In the upper panels, the actual measured velocities are depicted. We used the average velocities at each measured height as well as the additional velocity at the ceiling to fit a parabola using Matlab. From the fits, we extracted both the maximal velocity as well as estimated the shear rate directly above the substrate. The results can be found in Table 7.5. Following, we repeated the simulations presented above to accommodate the changed geometry for this particular experiment to evaluate the theoretical flow profile. To facilitate the direct comparison between the velocity parabolas, the discreet values were extracted from Comsol and fitted 
A
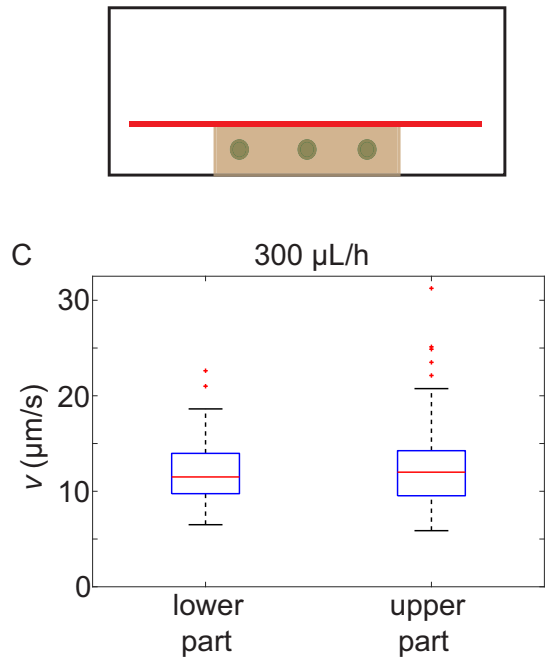

B

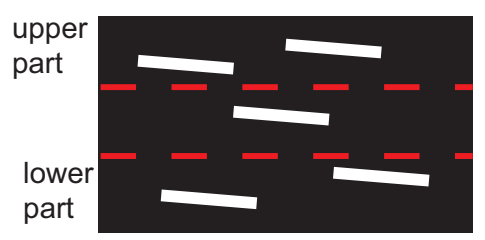

D

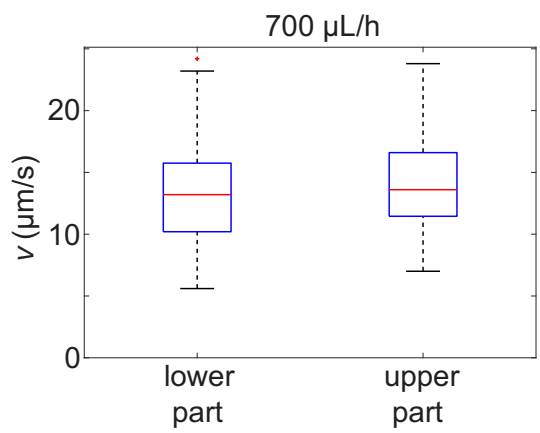

Figure 7.11.: A A cross section through the measuring chamber. To determine the flow profile on the substrate surface, multiple images were taken along its surface, denoted by the red line. $\boldsymbol{B}$ The resulting images of the bead traces (white lines) were analysed by dividing the images into three parts along its length (red dashed lines). Note that the long sides of the image point towards the side walls of the channel. The upper and lower parts were evaluated separately from each other towards the particle velocity in 50 separate images. The result for each part is shown for $\boldsymbol{C} 300 \mu \mathrm{L} / \mathrm{h}$ and $\boldsymbol{D} 700 \mu \mathrm{L} / \mathrm{h}$. No significant difference could be seen. 


\begin{tabular}{|l|c|c|}
\hline & $300 \mu \mathrm{L} / \mathrm{h}$ & $700 \mu \mathrm{L} / \mathrm{h}$ \\
\hline \hline Measured maximal velocity & $252 \mu \mathrm{m} / \mathrm{s}$ & $568 \mu \mathrm{m} / \mathrm{s}$ \\
\hline Simulated maximal velocity & $254 \mu \mathrm{m} / \mathrm{s}$ & $593 \mu \mathrm{m} / \mathrm{s}$ \\
\hline Measured shear rate & $13.9 \mathrm{~s}^{-1}$ & $32.3 \mathrm{~s}^{-1}$ \\
\hline Simulated shear rate & $14.5 \mathrm{~s}^{-1}$ & $33.8 \mathrm{~s}^{-1}$ \\
\hline
\end{tabular}

Table 7.5.: The measured and simulated maximal velocities for the flow profile shown in Fig. 7.12. From the parabolic fits calculated using Matlab, the shear rates above the substrates were derived.

with Matlab. The resulting fits are found in the lower panels and the estimated maximal velocities as well as shear rates in Table 7.5. Both from the graphical representation in Fig. 7.12 as well as the values listed in Table 7.5, it became clear that the flow within the chamber corresponded very well to the theoretically predicted one. The maximal velocities differed less than $5 \%$ for both cases. The same was true for the shear rates, which was the most interesting physical quantity in our experiments. Additionally, if we compare the estimated shear rates of this particular experiments with the given geometry to the average experiments simulated previously, we found that the difference was well under $5 \%$. Hence, it was concluded that even if smaller changes in the geometry occurred during the experiments, the shear rate did not differ by much. Note that most substrates used did exhibited a height of $25 \mu \mathrm{m}$ to $35 \mu \mathrm{m}$, the variations in the chamber height being of the same scale. We concluded that the theoretical predicted shear rates were in excellent agreement with the actually found shear rates, even including variations in the physical heights of the single components. Additionally, the expected flow profiles both horizontally as well as perpendicular to the gel surface were met in practice.

The experiments so far demonstrated the practical usability of the set-up with respect to the flow characteristics. The next step consisted of testing whether the platelets attached to the substrate. One has to keep in mind that our system was much simplified from a biological point of view. Under physiological conditions, more proteins are involved in the entire process than just fibrinogen and thrombin. Thus, we tested if these two proteins were sufficient to facilitate an attachment within our system. 

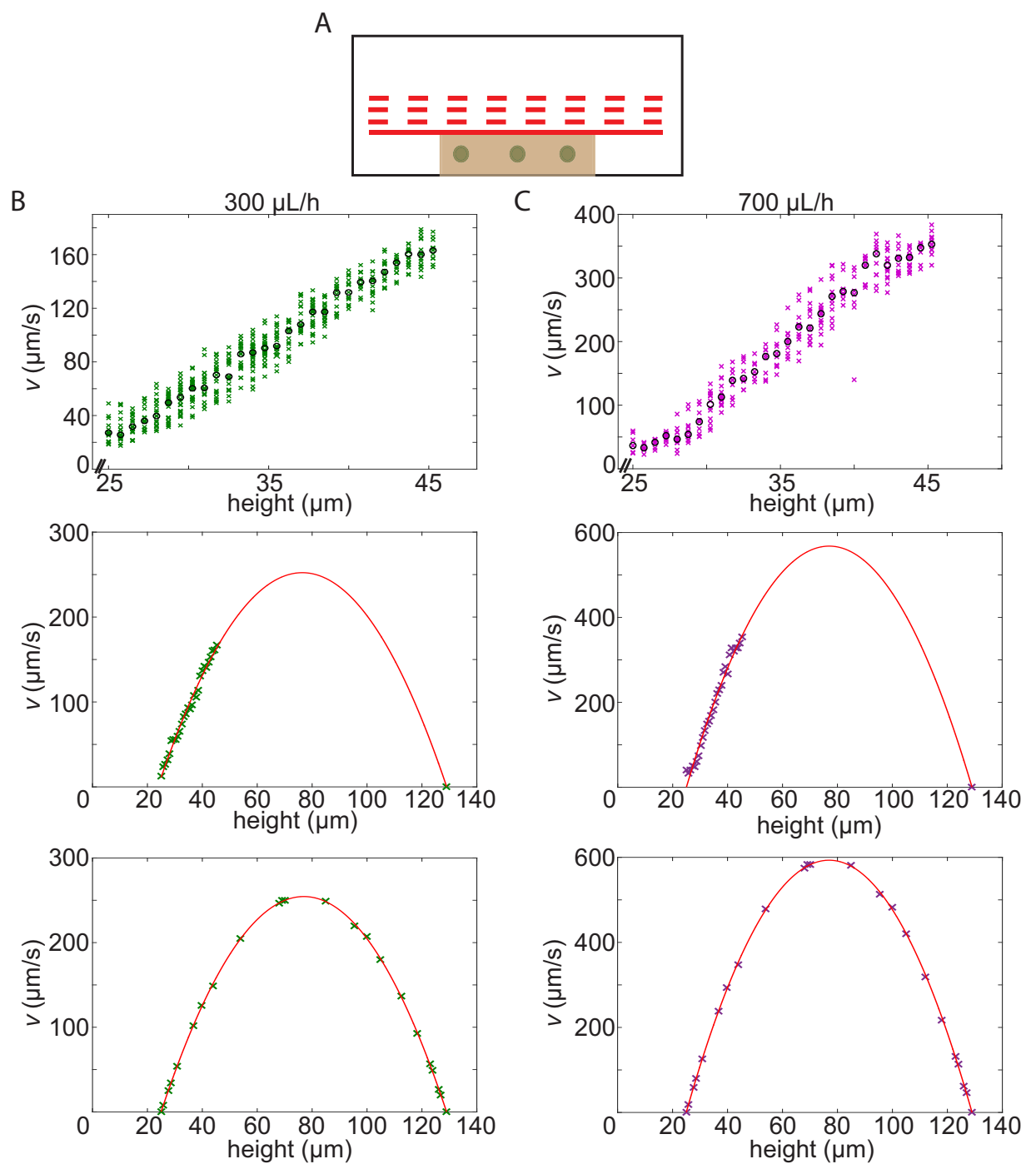

Figure 7.12.: Starting at the gel surface at $25 \mu \mathrm{m}, 28$ images in height were taken according to the sketch in $\boldsymbol{A}$. The first image was taken directly on the surface (red solid line) and than varied in height to produce equidistant images (red dashed lines). The results for $300 \mu \mathrm{L} / \mathrm{h}(\boldsymbol{B})$ and $700 \mu \mathrm{L} / \mathrm{h}(\boldsymbol{C})$ are shown here. The first row denotes the actual measured velocity values with their median. Subsequently, for each height, the mean velocity was calculated. From the measured, averaged values (crosses), a parabola was fitted (red line) using Matlab, shown in the middle row. The theoretical velocity profile determined with Comsol can be seen in the lower row. Here, the crosses mark the actually calculated values by Comsol and the red parabola fit was determined with Matlab. The difference in the maximal velocity between the simulated and measured velocity is less than $5 \%$. The image is taken from Hanke et al. [37]. 


\subsubsection{Attachment of Platelets During Flow}

To test the adhesion of the platelets to the substrate under flow, the experiment was conducted with the flow rates and concentrations as later used during the measurements. The attachment and possible contraction was monitored over $1.5 \mathrm{~h}$. At the end of each recording, the last image of the attached platelets was taken and all spread cells were evaluated as to their time point of attachment and whether they contracted. This analysis gave answers to three important questions:

1. Do the platelets attach under flow?

2. If so, when do they attach?

3. Do the platelets that attach also contract?

The second question was eminent to decide whether a recording time of $1.5 \mathrm{~h}$ was reasonable or had to be shortened or lengthened.

In Fig. $7.13 \mathbf{A}$, an example of an attachment test is seen. For this particular example, the flow rate was set to $300 \mu \mathrm{L} / \mathrm{h}$ and the last image of the recording is shown. All platelets that adhered and contracted were put into three categories: cells that attached as clusters, platelets that adhered separately but had other cells attaching to them at a later time point, thus forming clusters, and platelets that remained single during the entire recording process. For the measurements to determine the contraction force of a platelet, the first group was discarded, cells in the second group were only analysable until another cell attached to the original platelet while the last group was analysable in its entirety. Additionally, for this test, the approximate time of attachment was noted for all platelets. The example presented here corresponds to an average situation observed in terms of the attachment rate. While all tests conducted for flow rates between $300 \mu \mathrm{L} / \mathrm{h}$ and $700 \mu \mathrm{L} / \mathrm{h}$ saw some degree of adhesion, the rate varies greatly, from 4 to nearly 40 cells or cell clusters after $1.5 \mathrm{~h}$ of recording. At the same time, as can already be assessed from this example, out of all adhered platelets, the amount of clustering platelets were equally as often observed as single, contracting platelets. In Fig. $7.13 \mathbf{B}$, the relative attachment rate according to the time point of adhesion for all experiments is shown, a total of 202 platelets and platelet clusters taken from 5 independent experiment days. We observed that the majority of platelets attached between $5 \mathrm{~min}$ and $35 \mathrm{~min}$ after recording started. In the following $15 \mathrm{~min}$ interval, the attachment rate remained near constant before decaying further. To ensure that the bulk of platelets were recorded for about $30 \mathrm{~min}$, a recording time of an hour was sufficient. We still chose to record for $1.5 \mathrm{~h}$. The reason here was the aforementioned partially low attachment rate. By increasing the recording 
time for another $30 \mathrm{~min}$, we increased the probability to record more platelets as we also included the group adhering at the small plateau found between 35 min and $50 \mathrm{~min}$ in case of a reduced attachment rate.

Apart from the already mentioned flow rates, a higher flow rate of $1000 \mu \mathrm{L} / \mathrm{h}$ was also tested. All of the lower flow rates worked well concerning the attachment and contraction as presented above, however, the highest flow rate did not show any adhesion. The only attachment observed was found if an air bubble developed near the recording site which redirected the flow, effectively reducing the velocity. An example of the attachment due to the change in flow direction is shown in Fig. 7.14. This observation was previously reported in a precursor of the used flow chamber by C. Ranke [96], where the flow needed to be stopped to facilitate attachment. Hence, only the aforementioned three flow rates, $300 \mu \mathrm{L} / \mathrm{h}, 500 \mu \mathrm{L} / \mathrm{h}$ and $700 \mu \mathrm{L} / \mathrm{h}$, were employed to study the contraction of platelets under low shear rates.

\subsubsection{Contractile Behaviour Under Shear Flow}

The contractile behaviour of the blood platelets under flow conditions was investigated for the same physical properties as previously reported for the static experiments. These included the temporal force development, the traction force distribution, the maximal force compared to the spread area as well as the force dipoles. All data shown and discussed in this section are always presented together with the results from the static case with the same substrate stiffness of $41 \mathrm{kPa}$ for direct comparison. For the flow experiments, on average 22 platelets were recorded for each flow rate, sampled over 3 independent experimental days.

The temporal force development exhibited similar behaviours as reported for static conditions. Platelets featured either a force plateau, relaxed after initial contraction or started to oscillate after contraction, as seen in Fig. 7.15 A. Combination of all behaviours were again observed. However, pro rata, more cells started to relax than in the static case with a percentage of $45 \%, 30 \%$ and $25 \%$ for plateauing, relaxing and oscillating platelets, respectively, including double counting (static case: $49 \%, 18 \%$ and $32 \%$ ). A possible reason for this phenomenon was the prolonged observation time which increased by a factor of three. Additionally, although oscillations were well distinguishable in the force curves as well as in the single recordings, these oscillations were more repressed compared to a non-flow environment. Performing the Fourier analysis as described in Section 6.2.2 to determine the dominant oscillatory frequency showed a considerably lower relative 


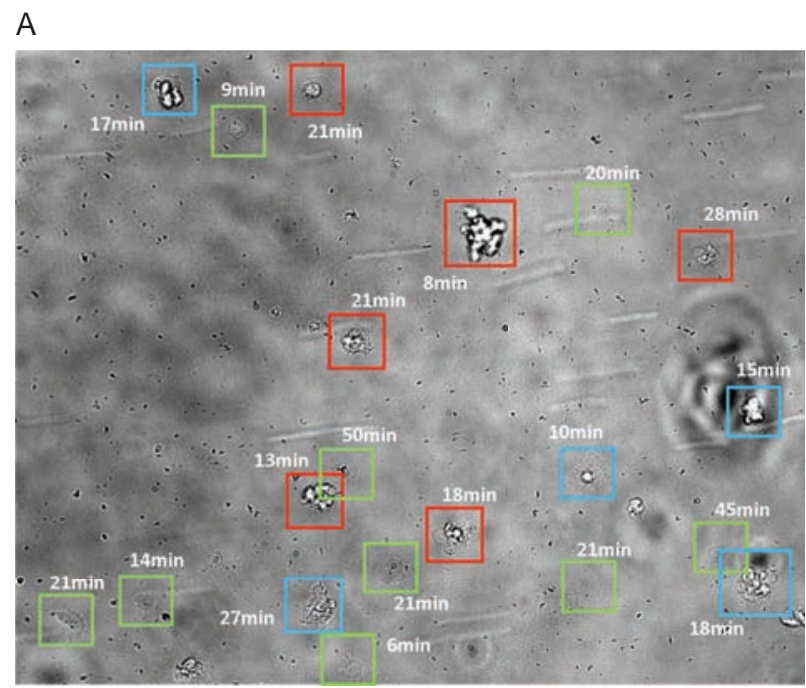

B

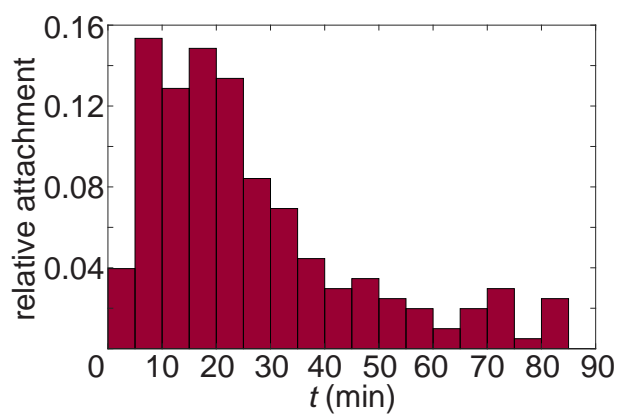

Figure 7.13.: $\boldsymbol{A}$ An example of an attachment test taken with a flow rate of $300 \mu \mathrm{L} / \mathrm{h}$. Green boxes mark platelets that attach and contract as a single cell. Blue boxes are platelets that attach as single cells and contract before other platelets attach to them. Red boxes denote contracting clots. All cells are marked as to their attachment time point relative to the start of recording. The streaks within the image are bypassing cells. The total recording time were $1.5 \mathrm{~h}$. $\boldsymbol{B}$ The time points of attachments of all experiments combined. Most platelets attached between 5 min and 35 min after recording started. Between 35 min and $50 \mathrm{~min}$, the attachment rate remains the same before decaying further. 


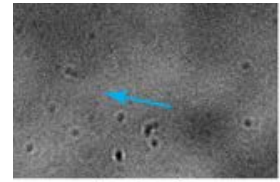

$\mathrm{t}=0 \mathrm{~min}$

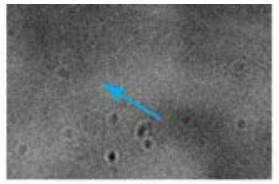

$\mathrm{t}=38 \mathrm{~min}$

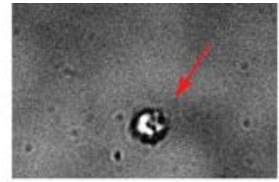

$\mathrm{t}=43 \mathrm{~min}$

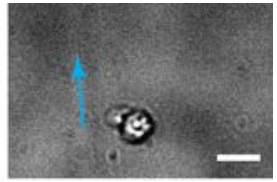

$\mathrm{t}=52 \mathrm{~min}$

Figure 7.14.: A time series of a recording at $1000 \mu \mathrm{L} / \mathrm{h}$. Here, the flow direction changed during recording, denoted in the image by the blue arrow. At about $38 \mathrm{~min}$, an obstacle outside the frame of recording redirected the flow, after which a cell attached at about $43 \mathrm{~min}$ (red arrow). At $52 \mathrm{~min}$ of recording, the flow has changed its direction by nearly $90^{\circ}$. The direction of the flow was derived from the entire recording by studying the traces of the by-passing cells.

A

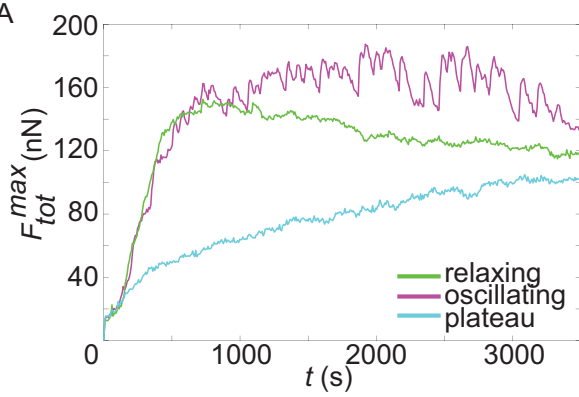

B

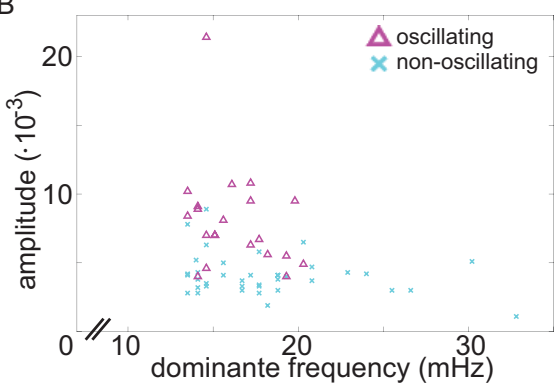

Figure 7.15.: A As in the static case, all three contractile behaviours were again observed. The three total force curves shown here were recorded for a flow rate of $500 \mu \mathrm{L} / \mathrm{h}$. $\boldsymbol{B}$ The dominant frequency and its corresponding amplitude of all recorded platelets under flow conditions. Less platelets displayed an oscillatory behaviour and their relative amplitude in Fourier space was considerably less than in the static case. Panel $\boldsymbol{A}$ is part of Ref. [37].

amplitude in the frequency spectrum (Fig. 7.15 B) compared to the static case depicted in Fig. 7.3 D. As the data contained in the Fourier analysis for the static conditions only amounted to, at max, 30 min of recording, the corresponding analysis for the flow experiments also only included, at most, $30 \mathrm{~min}$ of the force data after initial contraction of each platelet. Note that the total percentage of oscillating vs non-oscillating platelets was determined to be $34 \%$ and $66 \%$, respectively. This marked a reduction from the static case where we reported a percentage of $39 \%$ vs $61 \%$.

To compare the exerted forces of the platelets for different flow rates to each other, the total force curves were averaged. For each flow rate, the time point of attachment of each cell was set to $t=0$ and all data averaged until half of the platelets did no longer contribute to the force. The averaged forces with their 
A

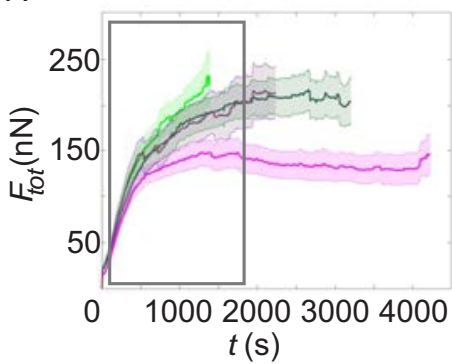

B

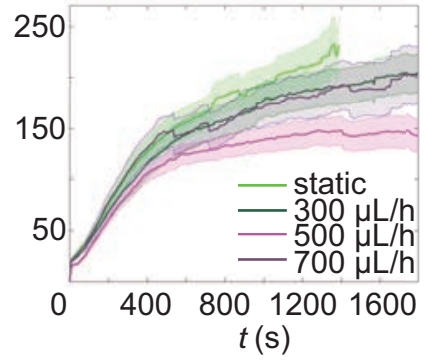

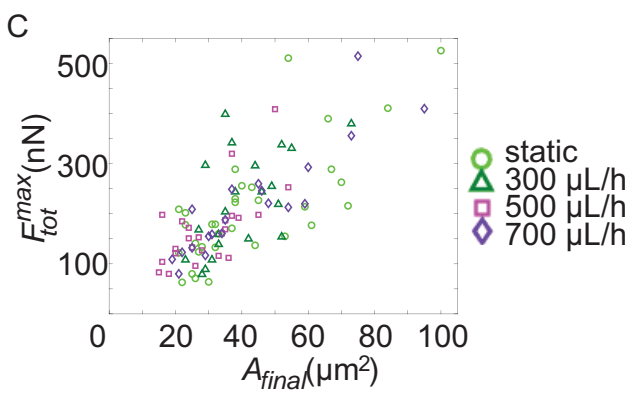

Figure 7.16.: A The calculated average total force curves over time for different flow rates. The bold lines denote the averaged force, the transparent areas the standard error. The first $30 \mathrm{~min}$ of recording (gray box; corresponding to the recording time of the static case) are separately seen in panel $\boldsymbol{B}$. $\boldsymbol{C}$ shows the dependency of the maximal exerted total force of each platelet on the final spread area. The figure is taken from Hanke et al. [37].

corresponding standard error, including the static case, are shown in Fig. 7.16 A. Taking the standard error around each graph into account, the magnitude of the shear rate acting on the platelets did not influence their total force. This became particularly clear if solely the first $30 \mathrm{~min}$ were investigated in greater detail (panel B). Keep in mind that the cells under static condition were only recorded for half an hour which made this time interval the most interesting for our considerations. Furthermore, a similar behaviour in the total force was observed when examining the maximal total force compared to the final spread area as seen in Fig. 7.16 C. As reported previously, with increasing spread area of the platelet, the maximal exerted force also increased monotonously without any difference between the different flow rates. Alongside the rising force, a larger variance was observed. 


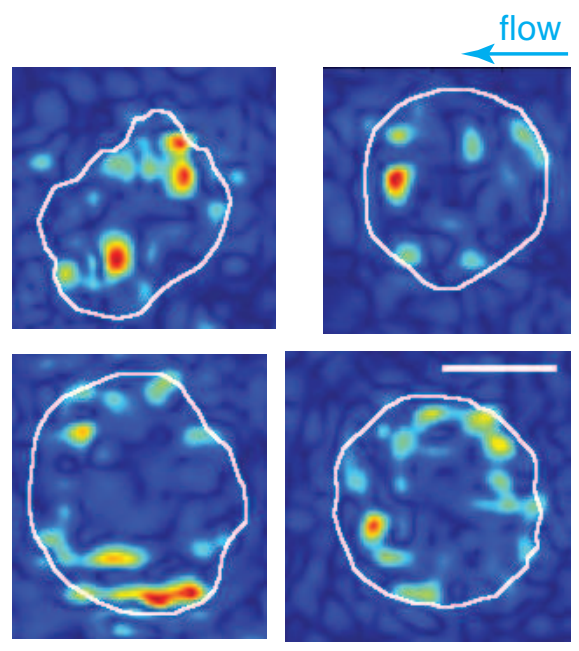

Figure 7.17.: Four examples of the traction force distribution and the platelets spread area. The cell outline is shown in white. The flow direction is denoted with the blue arrow. Scale bar: $5 \mu \mathrm{m}$. The images are partially taken from Ref. [37].

Up until this point, we have only investigated the total force curves. Let us now take a look at the traction force distribution for any possible adaptation to the shear rate. First, we observed that as previously shown in the static experiments, the traction forces were again concentrated at the periphery of the cell outline (compare Fig. 7.17). The number of force hot spots were not observed to be as discrete as previously reported when no shear flow was applied but more smoothed our in space. Still, no platelet contracting along the entire circumference were noted.

Next, the force dipoles were calculated as given in Section 6.2.3. An example is depicted in Fig. 7.18. We started by investigating the behaviour and relationship between the dipole moments over time. The dipole moment magnitudes were determined to develop largely synchronised to each other as reported before (see panel $\mathbf{B}$ and $\mathbf{C}$ ) apart from some highly polarised cells. We concluded that the final structure of the force transmitting network was established at the time point of attachment and did not vary during the time of recording. Next, we compared the dipole quotients $q$ between the different flow rates. The ratio for each platelet was averaged according to their variance as described in Section 5.5.2. Here, we did not detect any significant difference between the single flow rates. This indicated that no adaptation to the shear flow in the cytoskeletal structure in 
A

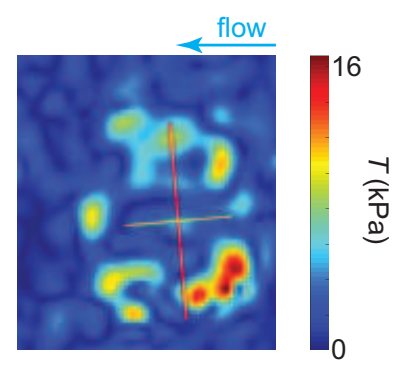

C

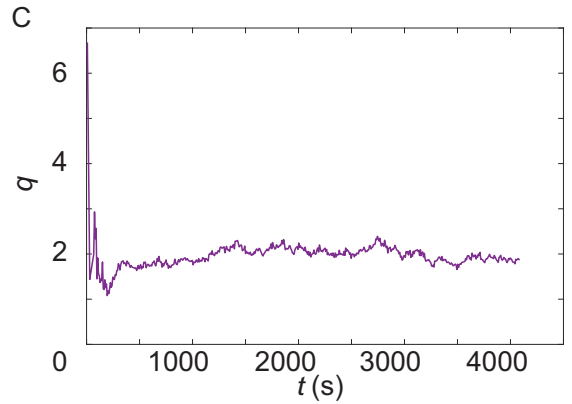

B

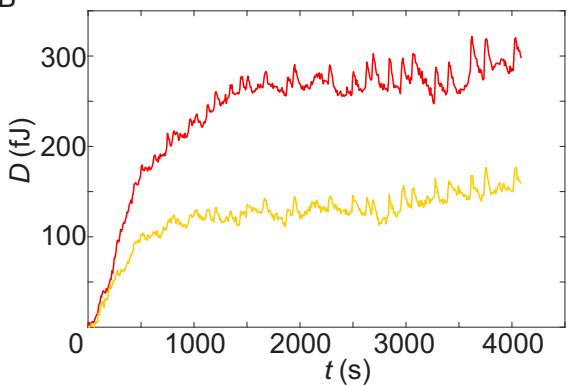

D

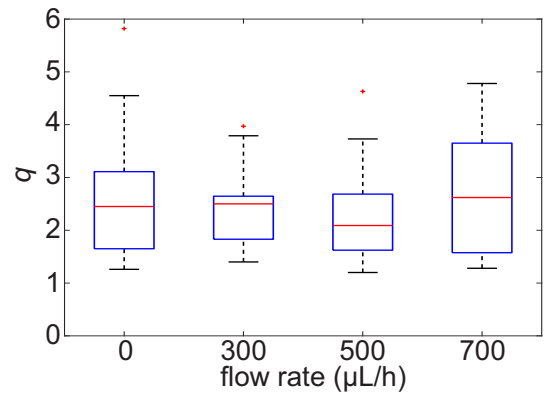

Figure 7.18.: An example for the development of the dipole moments for an oscillating platelet. In panel $\boldsymbol{A}$, an overlay of the traction force magnitude and the dipole axes is shown. The major dipole axis is depicted in red, the minor dipole axis in yellow. The length' of the axes are scaled according to their magnitude. The magnitude of the dipole moments over time can be seen in panel $\boldsymbol{B}$ and their quotient $q$ in $\boldsymbol{C}$. The averaged dipole quotients of all platelets divided according to the flow rates are depicted in panel $\boldsymbol{D}$. As can be seen, no difference between them could be observed. Panel $\boldsymbol{D}$ is part of Ref. [37].

terms of organisation did occur. Note that due to their low number and the previously demonstrated equality in the average dipole quotient, oscillating platelets were treated the same as non-oscillating platelets. All statistical tests here were conducted by the Wilcoxon rank sum test.

As we were not able to discern any difference in the aforementioned characteristics, we investigated whether the platelets adapted on another scale. It has previously been demonstrated that endothelial cells adapt to external shear flow by orienting their direction of contraction with the flow, although different angles have been reported at this stage [7]. Thus, let us have a look at the contraction direction of our platelets and if they vary their orientation according to the flow direction. 


\subsubsection{Adaptation of Force Orientation to the Shear Stress}

Let us first define the direction or orientation of contraction. From our previous considerations, we saw that the traction force distribution did not exhibit one single force orientation, i.e. two point forces directed towards each other, but contracted along the periphery into the centre of the cell. So the direction of contraction was defined as the preferred orientation of contraction in terms of an axis through the traction force distribution along which the majority of the forces concentrated. This essentially corresponds to the major axis of the dipole moment as defined by Eq. (3.14). Hence, the angle between the platelet contraction and the flow was defined as the angle between the major dipole axis and the direction of the flow. They were determined as described in detail in Sections 6.2.3 and 6.2.4, respectively.

To start with, consider the angle of the flow within the studied frame. During our analysis, we assumed that the flow was constant in direction and velocity over time. Larger changes in the orientation of the flow indicated an obstacle in the flow such as air bubbles. Data sets that actually displayed this kind of behaviour were excluded from analysis. However, from the data sets that were utilised for analysis, it was demonstrated that the flow over time was stable in direction as seen in the example depicted in Fig. 6.7. The deviations in the calculated angles over the entire recording time of $1.5 \mathrm{~h}$ were less than $10 \%$ and stem from the noticeable thickness of the cell traces in the bright-field images. For this and all following analyses, we set $0^{\circ}$ along the positive $\mathrm{x}$-axis, i.e., the right edge of the image. The flow was always directed towards the negative $\mathrm{x}$-axis, i.e., the flow direction was generally about $180^{\circ}$.

Next, the angle of the major dipole axis was calculated and, subsequently, the angle between flow and dipole axis. We previously argued in Section 6.2.4 that due to the constant velocity profile in horizontal direction, all angles were mapped to the interval between $0^{\circ}$ and $90^{\circ}$. In Section 7.3.2, we also proved that the velocity really was, in fact, equal parallel to the substrate surface. Calculating the opening angles between the major dipole axis and the flow direction for all flow rates yielded the results depicted in Fig. 7.19 B to D. Interestingly, contrary to all other aspects we investigated, an adaptation to the shear rate was observed. Especially, while the angle at the two lower flow rates demonstrated a preferred angle around $45^{\circ}$, at the higher flow velocity, this changed to an angle close to $90^{\circ}$. The increase in angle was determined to be significant, $p<0.03$, using the Wilcoxon rank sum test. Furthermore, we showed that the reported effect did not 
A

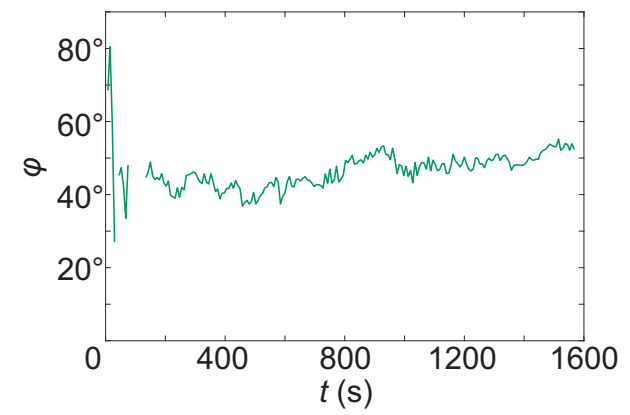

C

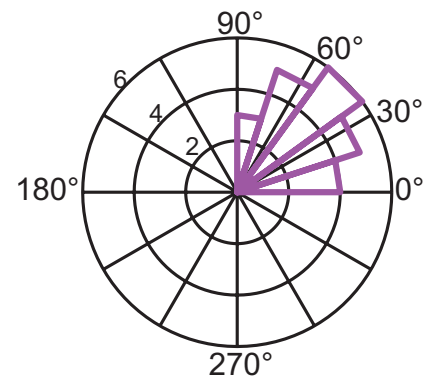

B

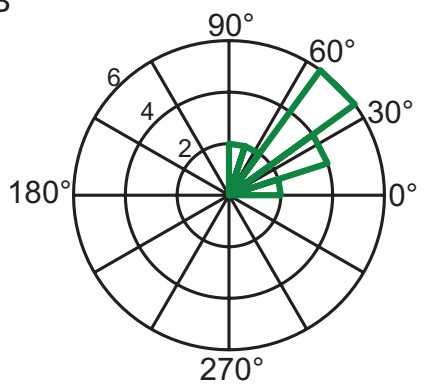

D

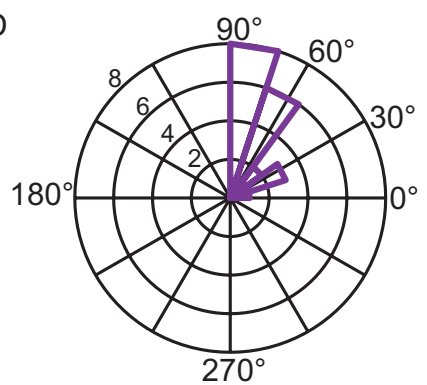

Figure 7.19.: A An example of the calculated angle between flow direction and the major dipole axis, measured at $300 \mu \mathrm{L} / \mathrm{h}$. As can be observed, the angle is comparably stable over time. The rose histograms of the angles for the flow rates $300 \mu \mathrm{L} / \mathrm{h}, 500 \mu \mathrm{L} / \mathrm{h}$ and $700 \mu \mathrm{L} / \mathrm{h}$ are shown in panels $\boldsymbol{B}, \boldsymbol{C}$ and $\boldsymbol{D}$, respectively. The figure is taken from Hanke et al. [37].

depend on the averaging over time as the angle was near constant over the entire recording time as shown in Fig. 7.19 A. In the averaging, we only included angles that coincided with dipole ratios that were also considered in the dipole averaging to guarantee a reliable result.

As mentioned in Section 6.2.4, we still had to keep in mind that isotropic contraction could cause a jumping angle over time. However, as can already be assessed from a closer inspection of Fig. 7.18 D, platelets exhibiting an entirely isotropic contractile behaviour were not observed. Hence, the analysis approach chosen worked well for our study.

To investigate the reason for the changes in the angle of the dipole axis, we simulated the stresses acting on the platelet when attached to the substrate as well as the stresses found within the cell. As the angle of the dipole towards the flow was near constant over time, we assumed that the stress distribution at the time 
of attachment determined the degree of adaptation. Hence, the geometry for the simulation contained an un-spread platelet within the channel. The un-spread cell was approximated by a spherical cap with a height of $1 \mu \mathrm{m}$ and a radius of $1.5 \mu \mathrm{m}$. Note that the platelet is very small compared to the dimensions of the entire channel as listed in Table. 7.3. Thus, the geometry was shortened in length to a total length of $200 \mu \mathrm{m}$ which was sufficiently long to establish a laminar flow profile before encountering the cell. In width, the entire chamber of $4 \mathrm{~mm}$ was considered to gain a complete picture of the stresses around the cell. Keep in mind that asymmetries in the stress distributions are very implausible due to the symmetry of the flow profile as shown and discussed above.

At the time point of adhesion, the platelet still exhibits a spherical shape. Although not exceedingly high, it locally changes the flow profile and thus the fluid shear stress distribution. Due to its elasticity, the platelet deforms in accordance to the stresses it is exposed to, compare the sketch in Fig. 7.20 A. Hence, for a reliable simulation of the stresses acting on the cell, we chose to include the elastic deformations of the platelet under flow. The Young's modulus and Poisson's ratio were taken as noted in Table 7.1 , its density was set to $1.1 \cdot 10^{3} \mathrm{~kg} / \mathrm{m}^{3}$, slightly higher than the density of water. Note that we did not include the elastic reaction of the substrate itself, which we assumed to be a solid wall. The following results gained from the simulations are depicted in Fig. 7.20, once for a flow rate of $300 \mu \mathrm{L} / \mathrm{h}(\mathbf{B})$ and once for $700 \mu \mathrm{L} / \mathrm{h}(\mathbf{C})$ to allow for direct comparison. We always show the area directly around the cell, viewed from above. The included grid cross marks the centre of the platelet for orientation.

In Fig. 7.20 $\mathbf{B}$ and $\mathbf{C}$, we show in the upper panels the shear stresses of the fluid acting both on the cell and the substrate. Directly at the lower periphery of the cell, the shear stress is close to 0 and increases towards the top. The highest shear stress exerted on the platelet was estimated to $0.05 \mathrm{~Pa}$ and $0.12 \mathrm{~Pa}$ for the lowest and highest flow rate, respectively. From the shear stress distribution detected around the surface, we expected to find the highest displacement on the top of the cell. This was proven to be true as shown in the second row. The total displacement or deformation of the cell was, however, very small. Even for the highest flow rate, the deformation did not exceed $60 \mathrm{pm}$. Most interestingly for our problem, namely the different contraction angles observed during flow, is the fact that we did not detect any difference in shear stress around the lower circumference of the platelet. A preferred contraction angle of $45^{\circ}$ or even $90^{\circ}$ could not be explained just by the shear stress. 
A

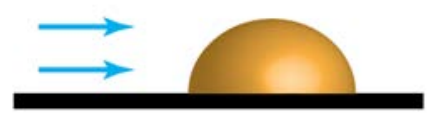

B
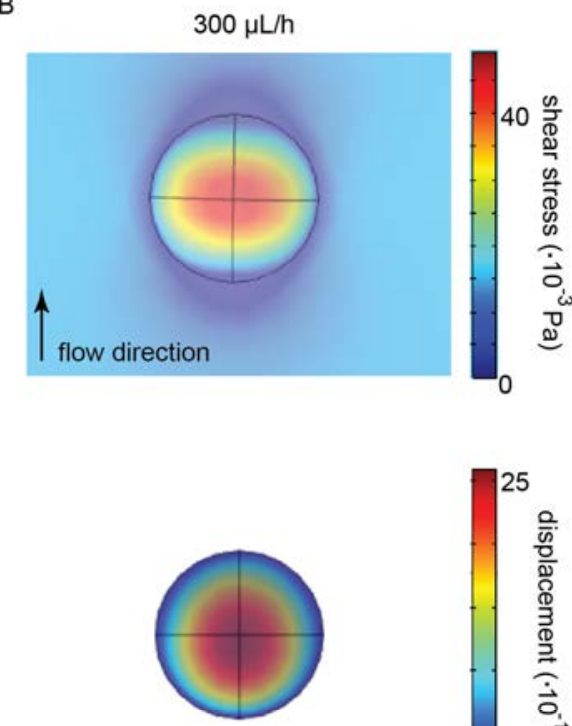

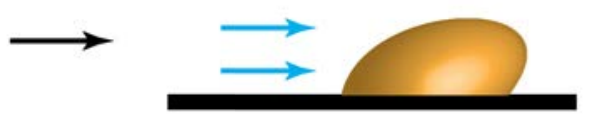

$700 \mu \mathrm{L} / \mathrm{h}$
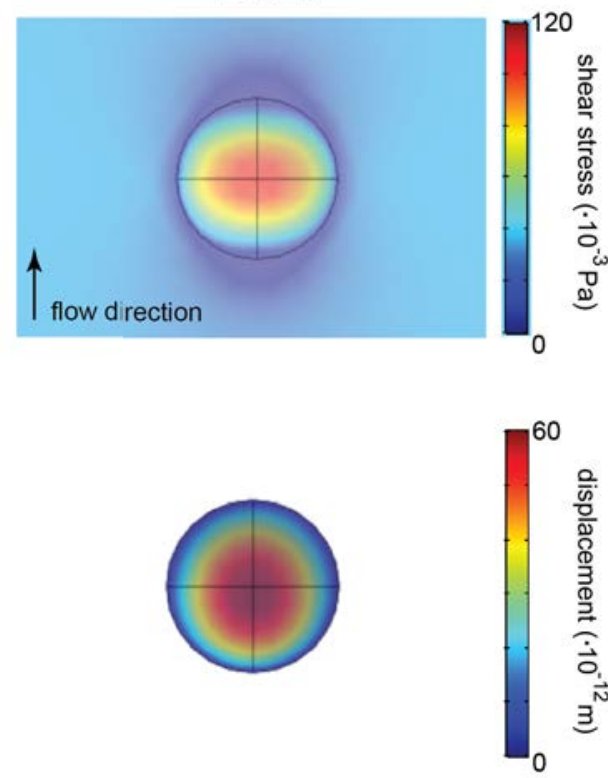

Figure 7.20.: A Upon exposure to shear flow (blue), the adherent, un-spread platelet (orange) deforms elastically. For the flow rates of $300 \mu \mathrm{L} / \mathrm{h}(\boldsymbol{B})$ and $700 \mu \mathrm{L} / \mathrm{h}(\boldsymbol{C})$, the shear stresses are shown in the upper row. The area around the cell is depicted as a top view. The highest shear stress is found on top of cell while at the adhesion site, the shear stress is close to 0 . In the lower panels, the corresponding deformation displacement is depicted. The deformation is in the picometer range and thus not visible in the simulations. The direction of the flow is added as a black arrow. Panel $\boldsymbol{C}$ is partially taken from Ref. [37]. 
To further investigate the stresses acting on the platelet under shear flow, let us consider the platelets as a elastic body connected to the substrate via small anchors, here the integrin proteins. These proteins act both as connectors as well as transmitters of the inner forces to the substrate. We only considered the preferred direction of contraction of our cells. Hence, we simplified the cell to an elastic body contracting along an axis and connected to its substrate at the endpoints of the axis (compare Fig. 7.21 A). If the force or stress on the proteins becomes too high, the connection fails and the cell detaches. In material science, to study possible failure, one calculates the von Mises stress as defined in Eq. (3.31). We performed this analysis on our attached platelet. Its results are shown in Fig 7.21 $\mathbf{B}$ and $\mathbf{C}$. Contrary to the shear stresses of the fluid, we now detected a break in symmetry from the front part to the back part of the cell. The difference in the stress distribution was a result of a discontinuity in the stress at the cell boundary. In particular, the highest stress, i.e. the point of most probable failure of the anchor proteins, was now found on the site directed towards the flow. The highest calculated von Mises stress was $0.36 \mathrm{~Pa}$ for $700 \mu \mathrm{L} / \mathrm{h}$. The lowest stress was found slightly behind the middle line stretching from $90^{\circ}$ to $270^{\circ}$. Note that the stress distribution of both flow rates shown did not differ, only their corresponding magnitudes. Going back to our simplification of the elastic geometry, to expose the anchors to the lowest stresses, both had to be placed behind the middle line. However, as our contraction was directed towards the centre of the platelet, the anchor points had to exhibit an angle of $180^{\circ}$ between them. Hence, a position around $90^{\circ}$ and $270^{\circ}$ still kept the stresses comparatively low and, additionally, equal on both sides. This explained the perpendicular orientation of the contraction at the higher flow rate. For the lower flow rate, the absolute stresses as well as the stress difference along the circumference was lower. We argue here that both the magnitude and magnitude difference of the von Mises stress was not sufficient to force an adaptation of the platelet to the flow. To ensure that our estimates were reasonable, we integrated the stress on the cell surface both parallel and perpendicular to the flow. For the highest flow rate, they amounted to $0.69 \mathrm{pN}$ and $0.65 \mathrm{pN}$, respectively. This is two to three orders of magnitudes lower than the previously reported detachment force between integrin and fibrinogen [67]. Hence, from the simulations, we expected no detachment to occur which we also observed in practice.

To summarise, we designed a microfluidic system, comprised out of two different devices, to investigate the contraction of blood platelets under flow. The first device facilitated the mixing of the platelets with its trigger substance thrombin in a 
A

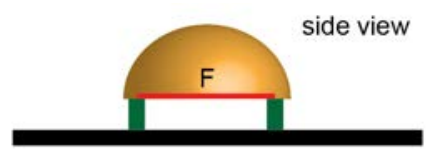

B

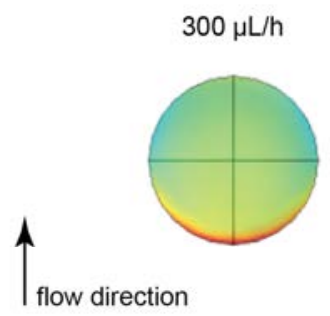

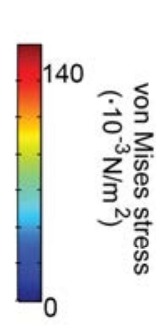

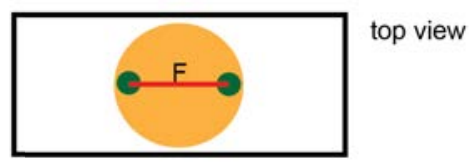

C

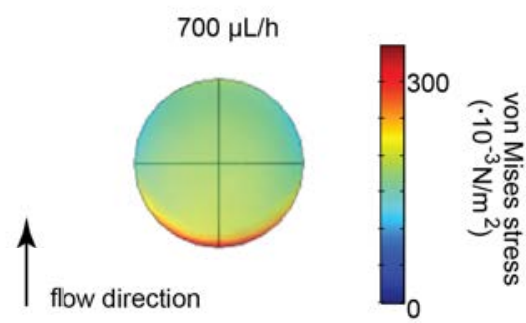

Figure 7.21.: A A sketch of our simplified contraction model, considering only the preferred direction of contraction. The cell (orange) is connected to the substrate (black) by anchor proteins (green). Between the anchors, a force is transmitted along a single axis (red). The model is depicted both from the side (left) and the from the top (right). The calculated von Mises stress is shown in $\boldsymbol{B}$ and $\boldsymbol{C}$ for a flow rate of $300 \mu \mathrm{L} / \mathrm{h}$ and $700 \mu \mathrm{L} / \mathrm{h}$, respectively, in a top view. The direction of the flow is indicated by the black arrow. The highest stress is found at the front of the platelet, the lowest stresses in the back half of the cell, directly behind the middle section. The image is partially taken from Hanke et al. [37].

controlled manner towards a desired concentration ratio between them. The second device was the measuring chamber containing the PAA substrate to enable the observation of the contraction. We characterised both devices both theoretically as well as experimentally and found them to be in good agreement. Additionally, we tested the chambers practical usability in terms of platelet adhesion and contraction. For the actual experiments, we employed flow rates that mimicked the low shear rates found in larger veins. We found that the platelets behaved similarly to their counterparts under static conditions in terms of total force, traction force distribution and the relationship between total force and final spread area. Interestingly, we detected an adaptation in the direction of contraction towards the flow. For the lower shear rates, the contraction exhibited an average angle towards the flow direction of about $45^{\circ}$, at the highest shear rate, we saw an angle of about $90^{\circ}$. By calculating the von Mises stresses, we found that the best position of the anchor proteins for our simplified contraction system was at close to 
$90^{\circ}$ in terms of lowest possible stress exposure. We concluded that this dictated the orientation of the contraction at higher shear rates while it did not influence the cells as much at lower shear rates. 



\section{Discussion}

In this chapter, we take a closer look at the results we described in the previous part. Here, we briefly consider the analysis algorithm we developed to be able to determine the forces exerted by the platelets and its impact on the calculated force (Section 8.1). Subsequently, the design of the microfluidic device is compared to previously reported chambers and its advantages discussed (Section 8.2). In Section 8.3, we first consider some general results concerning the contraction of platelets and their implications from both experiments before evaluating the observations of the oscillatory contraction (Section 8.4) and the force dipole orientation in a flow field (Section 8.5) in more detail.

\subsection{Analysis Algorithm}

In this thesis, we intended to measure the temporal evolution of human blood platelets when exposed to different external environments. We observed that these forces were not only fast changing over time but also large in magnitude while confined to the small area of the fully spread cell. Hence, we first had to construct an analysis algorithm that allowed us to evaluate recordings of the contractile cells. As described in Chapters 5 and 6, we derived a carefully tailored algorithm based on differential PIV to calculate the highly dynamic traction forces of platelets. We also compared the PIV approach to a differential optical flow method. Both PIV and optical flow have been used before to study cellular traction forces $[3,45,46$, $87,110]$ but were mostly used for larger cells, often with lower forces and, in several cases, not time-resolved. These approaches however failed for our application due to the high, spatially confined forces. To illustrate this point, consider two different primary cells, namely endothelial cells and dictyostelium cells. Endothelial cells have been shown to exhibit about the same total forces as we reported here for the platelets while being one to two orders of magnitude larger in area [12,35]. Dictyostelium cells on the other hand have roughly the same size as platelets but only exert a total force of about a tenth of platelets [20]. 
As both algorithms used had to be adapted to a differential approach, they were calibrated against each other to exclude an algorithm-dependent result. We demonstrated that both algorithms yield similar results within the total force although the traction force maps differed slightly between them. While the traction forces were more confined in space but higher in magnitude when employing the PIV method, the traction forces calculated with the optical flow method were more smoothed in space and lower in magnitude. In Holenstein et al. [45], it was previously observed that PIV approaches yield lower traction forces compared to the optical flow method when investigating considerably larger cells than used here. However, we demonstrated in Section 5.4 that the need for regularisation is highly dependent on the kind of data one wishes to analyse, i.e. the behaviour of the wave vectors. It thus stands to reason that the differences in the results of the different algorithms vary largely with the resolution and physical size of the problem under investigation. Note that in our case, despite their local differences, the overall pattern in the traction forces were still comparable. In particular, the positions of the force maxima remained the same independent of the algorithm utilised.

While both algorithms have been proven to yield the same results in terms of total force, the results were higher than the values of previously reported forces. In Schwarz Henriques et al. [107], platelets were recorded on $4 \mathrm{kPa}$ PAA substrates and the forces measured to be about $40 \mathrm{nN}$. Here, PTV was employed to track the deformations in the gel over time. In the current work, such direct tracking was not possible due to much higher bead density. Indeed, comparing the resolution due to the number of beads per area, in the present work, we have at least one order of magnitude more information in terms of moving particles than in Ref. [107]. Thus, it is well possible that forces were underestimated using the lower resolution. Note that the chemical set-up of the experiments conducted by Schwarz Henriques also differed in some aspects. The chemical aspects of this and other experiments and their influence on the contraction are discussed separately further down.

In another approach of using atomic force microscopy, Lam et al. [58] also reported lower forces of, on average, $20 \mathrm{nN}$. Here, a one-dimensional approach was chosen by letting the platelet attach to a substrate on one side and to the tip of the cantilever on the other. The contractile force was then measured by the deflection of the cantilever from its resting point. As we observed in the previous chapter, however, the forces of the contraction of a fully spread platelet were distributed 
along the entire periphery. The two-dimensionality in the contraction was not considered in the approach using the atomic force microscope and thus might lead to an underestimation of the actual forces.

Similar to the previous study, Myers et al. [81] measured the forces of platelets when spreading on fibrinogen patterned substrates. Here, the forces of the platelets were calculated by letting them adhere between two fibrinogen patches and observing the deflection of these spots. As with the approach using atomic force microscopy, this reduced the problem to an uni-axial problem which may not capture all details found in two-dimensions. Using this method, they determined a total force of up to $70 \mathrm{nN}$ on average in dependency of the stiffness and thrombin concentration.

To estimate whether our reported forces were unreasonably high, we approximated the maximal expected total force by the amount of acting myosin motors and the force each of them can exert. According to Ref. [54], it was measured that a single myosin motor produces a power stroke of up to $10 \mathrm{pN}$. Previously, it was estimated that a single platelet contains about 12000 myosin motors [77]. This yields an upper bound of $120 \mathrm{nN}$ if all motors moved synchronised. While this force is in the same order of magnitude as we reported here, it is slightly lower than what we measured. Did we then overestimating the force? This is not necessarily the case. To determine the number of myosin motors per platelet, the molecules were extracted from a larger platelet clot to be estimated. Two problems with this approach need to be considered. First, the total number of single cells within the clot had to be estimated correctly. Additionally, the clot included cells of various size. In this work, we observed an average fully spread area of about $40 \mu \mathrm{m}^{2}$. Previously, on harder PDMS substrates, an area of $30 \mu \mathrm{m}^{2}$ [102] was reported. If we take this into account and the knowledge that generally, on very hard substrates the spread area tends to increase rather than decrease [93], we can conclude that on average, contracting platelets are larger than the mean over all platelets. Thus, it is well reasonable that the cells observed here contain a higher number of myosin motors than the average platelet. Additionally, a force is also exerted when actin is polymerised as observed during platelet spreading. It was previously reported for other cells $[11,46]$ that the type of cell determines the influence of the myosin-driven contraction $v s$ the actin-polymerisation-driven contraction. How large the influence of the single components on the contraction is for human blood platelets has not been investigated yet. But to assume that 
at least part of the observed force stems from the actin polymerisation is well justified. A measured force higher than the approximated $120 \mathrm{nN}$ hence does not indicate an overestimation of the force.

\subsection{Design of Microfluidic Device}

A major part of this work was the development of a microfluidic chamber that allowed us to study the traction forces exerted by the platelets under flow conditions. Here, we chose to embed a protein-coated PAA gel into a PDMS channel using a plasma binding technique. In particular, the PAA substrate was coated with fibrinogen before it was treated in the plasma cleaner. To avoid damage to the protein and gel, during plasma exposure, the substrate was covered by a thin PDMS stripe. After bonding, the channel and the connecting tubings were filled with water for storage. Depending on the storage technique, we were able to store the devices up to one week.

While the measurement of forces under flow conditions is still an emerging subject in research, some microfluidic chambers have already been described in literature. None of them were constructed similar to the one we used here although some come closer to our construction than others. Let us here have a look at other devices and illustrate why we chose the approach we did. The most interesting questions to ask are concerning the combination of substrate and PDMS channel as well as the choice of method for the protein-coating. We are also shortly discussing the usage of an external mixing device versus alternative approaches.

For combining the substrate and the channel device, we chose a method where we first produced the PAA substrate similar to the static experiments and separately cast a PDMS device as often done for different microfluidic devices and measurements. By employing a similar substrate than we had used prior for the static experiments, we were able to use already derived analysis methods as well as directly compare the results from both experiments to each other. The PDMS casting from a silicon waver we utilised to create the channel had the advantage that we were able to produce several copies of the channel with identical geometry by reusing the corresponding waver. These were fabricated in-house in a clean room. Here, the height of the wavers was controlled by different production protocols thus rendering the set-up potentially quite flexible. As an alternative, as done by Steward et al. [112], we could have incorporated the substrate into a commercial available microfluidic platform. However, this diminishes the variability of the geometry we achieved by fabricating the devices ourselves and the idea was thus 
discarded. Devices constructed by binding a glass slide to a self-made PDMS cast have been employed in other studies by Perrault et al. [92], Das et al. [17], Hur et al. [47], Myers et al. [81] and Lembong et al. [63]. For the binding, they either only oxidised the PDMS cast and press bonded the device [17], used customised holders to hold the chamber together [47,92], silicon adhesives [81] or bound the glass slide and PDMS before the PAA substrate was polymerised [63]. For a precursor of our chamber, we also tried to just activate the PDMS by plasma treatment [96]. However, these chambers proved to not be entirely leak-proof and generally did not sustain for the entire preparation and experimental time of several hours. Our alternative to prepare all components beforehand and oxidising both the glass and the PDMS cast while protecting critical regions proved to be much more reliable. Indeed, as long as no air pockets remained between PDMS and glass and no part of the substrate was underneath the PDMS, no problem with leakage was observed. At the same time, this procedure did not require any additional tools to facilitate binding. Moreover, an extra layer of adhesive would inadvisedly change the height of the whole channel, changing the flow conditions within.

The microfluidic device that came closest to our device is the one designed in terms of the assembly process by Lam et al. [57]. Here, they incorporated a micro-post array in a PDMS device. In particular, they coated the array with adhesion proteins before plasma treatment of both the glass containing the array and the PDMS cast. During plasma treatment, similar to us, the proteins on the posts were protected by a piece of PDMS which was removed before binding. The prominent difference between their technique and our method was the use of the force measurement method. As we mentioned previously, the disadvantage of a micro-post array is that data may only be collected at discrete points in space contrary to continuous substrates. While this works well for larger cells which still adhere to a sufficient number of posts, this does not hold true for the smaller platelets. An additional effect to considered in the set-up used by Lam et al. is the distortion of the flow field. As they simulated for their device, it was shown that the flow field along the posts was not constant in space in contrast to our device. They demonstrated that the shear stress was considerably higher on the posts than in between them. For our device, the flow field along the gel was constant, guaranteeing that attached platelet experienced the same shear stress along its entire circumference only distorted by its own geometry. Hence, we argue that our flow regime resembles more the actual conditions within the body than a shear 
stress system of larger unevenness as seen in micro-post arrays. For comparison, their chamber had the same height as our chamber $(100 \mu \mathrm{m})$ while their posts were $9 \mu \mathrm{m}$ high.

The next question to consider is why we chose to add the protein before the plasma treatment instead of after the binding of the chamber. Indeed, for the binding of fibrinogen to the substrate, it did not mattered whether the gel underwent plasma treatment first or not. In literature, both approaches exist of either first coating the substrate with protein or first assembling the chamber. The former method was mostly used in the chambers that we described previously containing some type of substrate or micro-contact patterning $[18,39]$ while the latter method was mostly utilised when handling plain glass slides $[34,83]$. The most important reason to not coat the substrate with fibrinogen after the binding was that fibrinogen reacts to high shear forces. When exposed to high forces, fibrinogen elongates and changes its elastic properties $[4,68]$, an effect which does not reverse directly after force reduction. After binding, to fill the chamber with protein solution, the solution had to be injected via a thin tubing, considerably thinner than the actual chamber. Hence, the shear stresses within the tubing are higher than what we simulated for the chamber. We could thus not exclude that the fibinogen would react to the high shear stress within the tubing. To avoid any unnecessary stresses, all fibrinogen solutions were handled with utmost care. This included the usage of as large pipettes as possible, no vortexing during mixing and no flow through thin tubes. Hence, coating before the plasma treatment was chosen. This also resulted in an easier handling of the coating step.

When we investigated the adhesion of the platelets to the substrates, we observed that the platelets did not attach when choosing a flow rate of $1000 \mu \mathrm{L} / \mathrm{h}$, corresponding to a shear rate of about $50 \mathrm{~s}^{-1}$. The same effect was reported by Ranke [96], where the flow had to be stopped to facilitate adhesion. Once the platelets were attached, the flow did not detach the platelets again. The same observation was done by Myers et al. [81], where a shear rate of $100 \mathrm{~s}^{-1}$ did not effect the platelets as long as they were already attached before the start of the flow. According to previous experiments [34,103], at this flow rate, the reaction to fibrinogen predominates the adhesion. Indeed, Van de Walle et al. [18] studied the attachment to spaced fibrinogen patches under a shear rate of $100 \mathrm{~s}^{-1}$. This raises the question why the platelets did not attach when exposed to a lower shear rate. Several aspects need to be considered here which all influences the experiments. First, the reaction between fibrinogen and the integrin $\alpha \operatorname{IIb} \beta 3$ is a comparably slow reaction (in the order of $\mathrm{ms}$ to s for stable binding [66]). Hence, 
to facilitate attachment, sufficient time has to be given for the reaction in terms of dwelling time. In the human body, it has been shown by experiment [1] and simulation [118] that the attachment is aided by the red blood cells actively pressing the platelets to the vessel wall and arresting them there. In our experiments, no such particles were included. Previous studies to the adhesion under flow, apart from Ranke [96], all used whole blood samples instead of purified blood plasma as we did here. Furthermore, all devices used in these works employed hard glass surfaces instead of softer substrates. As has been shown in other studies [93], platelets react much stronger to glass surfaces than softer substrates in terms of adhesion rate and spreading area. Softer substrates may well reduce the chances of attachment. While the natural environment in the human body is softer by nature than glass, other mechanisms help the attachment along here. As the platelets still attached to the substrates when we used a lower flow rate or stopped the flow, the problem did not lay in the protein coating.

Before continuing to our results, let us briefly remark on our choice of an external mixing device to combine the thrombin and platelets. We chose to activate the platelets by introducing thrombin into our experiments. To be able to compare our two different experiments, the concentrations between the components were kept constant. Hence, we had to inject the thrombin at the correct concentration into the platelet solution. This could be done by either using one syringe with the complete mixture, mixing before the measuring chamber or mixing within the device itself. The first approach was used by Ranke [96] and seemed to work quite well. However, the platelets were essentially already activated within the syringe, making it difficult to approximate the exact time between activation and attachment or start of the experiment. Additionally, the reaction time between the cells and trigger substance statistically increased with increasing recording time, i.e. platelets entering the chamber at a later time point were exposed longer to thrombin. For our static experiments, this time could actually be set to about $2 \mathrm{~min}$ plus some time for the diffusion of thrombin and platelet within the liquid. Mixing within the measuring chamber lead to the effect that platelets become activated and directly flushed out of the system. Hence, an external mixing device was chosen. This had the advantage that the reaction time between the two components was well approximated and controllable by the length of the connecting tubing between the mixing device and the chamber. 


\subsection{General Observation on Platelet Contraction}

Independent on the experiment we conducted, several aspects in terms of force development remained the same. One such aspect was the temporal development of the contraction over time where we distinguished between three trends: some platelets reached a force plateau, some relaxed after contraction and some started to oscillate after initial contraction. Further, the total force over time did not change if the environment changed in terms of substrate stiffness or shear rate within the studied ranges. In fact, the total force seemed to only depend on the size of the platelet. For the spatial distribution of the traction forces, we demonstrated that the force was transmitted at the periphery of the platelet. This transmission was generally not spread evenly over the cell's outline but often focused into two to four 'hot spots'.

To start, consider first the contractile behaviour over time. The oscillatory behaviour is discussed separately in a later section. To understand the other behaviours of a force plateau and the relaxation, let us go back to the mechanics of platelet contraction. As described previously in Section 2.1, when the platelet is activated, e.g. by thrombin, an increase in intracellular calcium is observed. This not only activates the $\alpha \operatorname{IIb} \beta 3$ integrin to facilitate further adhesion but also triggers the contraction. In particular, the myosin binds to the polymerised actin filaments and the platelet contracts. Hence, calcium is an important player in the signalling cascade of contraction. But how can the platelet increase its intracellular calcium concentration? On one hand, calcium is stored within the dense granules. On the other hand, in vivo, calcium is found in the blood. It has previously been observed that calcium flux occurs during activation and contraction [84, 128]. Additionally, ATP is needed for the actual power stroke of the myosin motor. ATP is also stored in the granules. Consider now our actual experiments. Going back to the compositions of the buffers (Table 4.1) we used for the platelets during isolation as well as recording, they contained neither calcium nor ATP. Hence, our investigated platelets had to relay on their own storages of the necessary molecules. While an exchange of calcium among the cells was well possible, the total amount of energy-giving molecules within the system was finite. We can thus interpret the force plateau as a platelet that first activated its myosin motors and then kept its calcium level constant and hydrolysed the available ATP at a steady rate over time. The relaxing platelets on the other hand did in all likelihood not contain enough molecules to exhibit prolonged contraction. Given this argumentation, one can conclude that all platelets sooner or later start to relax. For longer ob- 
servation times, there are hints that this is true. If we for example consider the longer recording time of the flow experiments, the percentage of relaxing platelets increased compared to the static case. This was best assessable in the averaged force curve of the flow rate $500 \mu \mathrm{L} / \mathrm{h}$ (Fig. 7.16 A), where the decrease was observable even after averaging. Indeed, a similar result was observed by Zhang et al. [128] under similar, static conditions where an abrupt decrease in the integrin tension was observed after $30 \mathrm{~min}$ to $50 \mathrm{~min}$ which was interpreted as a loss of contraction.

Next, we observed that the external environment did not seem to influence the force exerted by the single platelets. While the influence of an increasing shear rate on the total force has as of yet not been studied in detail, the substrate stiffness dependency has been investigated in depth. From cells such as fibroblasts [69], Jurkat T-cells [46], neutrophils [89] and endothelial cells [12], it is known that they do adapt their forces to the substrate rigidity underneath such that higher forces are measured on harder substrates. Here, instead, we did not detect any such kind of adaptation, neither in force nor area. A first thought was that platelets are not mechano-sensitive in the studied elasticity range. However, in Lam et al. [58], an adaptation in the force was observed when measuring the contraction with an AFM. Additionally, Qui et al. [93] saw an adaptation in the spread area according to the stiffness. However, taking a closer look at the latter publication, reveals that a plateau in area was observed on substrate between $50 \mathrm{kPa}$ and $100 \mathrm{kPa}$. A further increase in area was not observed until the cells were plated on glass. For the first paper, we already discussed the draw-backs of an uni-axial analysis in a previous section. Also here, the largest increase in force was detected on an infinite stiff cantilever and a smaller increase in force between $12 \mathrm{kPa}$ and $29 \mathrm{kPa}$. An interesting point is that both experiments were conducted using different concentrations of fibrinogen and thrombin. Qui et al. [93] employed a fibrinogen concentration of $20 \mu \mathrm{g} / \mathrm{mL}$ and no thrombin exposure while Lam et al. [58] chose a fibrinogen concentration of $3 \mu \mathrm{g} / \mathrm{mL}$ and a thrombin concentration of $1 \mathrm{u} / \mathrm{mL}$. Note that we used a concentration of $100 \mu \mathrm{g} / \mathrm{mL}$ and $4 \mathrm{u} / \mathrm{mL}$ for fibrinogen and thrombin, respectively. Qui repeated the experiments for a fibrinogen concentration of $100 \mu \mathrm{g} / \mathrm{mL}$ and found a stagnation in area and adhesion rate. At higher fibrinogen concentrations, the sensitivity to the substrate stiffness seems diminished which explains the constant force over all tested elasticities. If, however, the concentration of the adhesion protein plays a larger role in the force adaptation than the mechanical environment, we also need to consider the concentration of the trigger substance. We used thrombin to activate the platelets as is also found within the 
body upon injury. The concentration in the human body can vary greatly from some picomolar to micromolar. The concentration we used here corresponds to a some nanomolar, so well within the physiological range. It has been shown in previous works that the activation effect of thrombin changes with the dosage but plateaus at about $3.5 \mathrm{u} / \mathrm{mL}$ for the reaction in the force measurements [65] and about $1 \mathrm{u} / \mathrm{mL}$ for the dynamics in the outer membrane [108]. Hence, we were within the saturation region in terms of thrombin concentration and assume that we activated the platelets to exert the highest force they were physically able to. Additionally, note that Schwarz Henriques et al. [107] also only employed $1 \mathrm{u} / \mathrm{mL}$ in the experiments which can be another reason why we saw a higher force than they did. The question than arises, whether the activation due to chemical cues does not override the adaptation to mechanical cues. Corresponding experiments have not been conducted within the scope of this work.

What we did observe was the dependency of the maximal exerted force on the final spread area. We demonstrated that with increasing area, the maximal exerted force also increased. A similar phenomenon was previously reported for endothelial cells $[12,35]$. Here, a linear dependency between the force and area was observed. For our data, a linear fit could not be used with good reliability. Instead, the model proposed by Edwards et al. [26] was found to give a better description of the dependency. From this model, we derived that within our stiffness range, the exerted force of platelets steadily increases until an approximately size of about $3000 \mu \mathrm{m}^{2}$, an unrealistic large area for platelets. Hence, such a stagnation of force is indicated to not exist from the model. An interesting point at this stage is, why the platelets scale their force with area but not with the stiffness of the underlying substrate. In contrast, endothelial cells do both. This can be explained by the presently most used model of the development of platelets ( [77], Chapter 2). As we stated previously (Section 2.1.1), platelets form by a budding-off process from larger megakaryocytes. This process, while regulated, is governed by statistical processes. On one hand, the size between platelets varies greatly as we saw in Figs. $7.4 \mathbf{A}$ and $7.16 \mathbf{C}$. On the other hand, this allows for a large diversity in chemical set-ups within the platelets, i.e. the amount of the single components such as myosin motors. A hint to this large variation can be assessed from the previously mentioned figures where the differences in exerted force by cells of equal size increased with increasing area. We thus argue that the increased force exerted by larger platelets is, among others, explained by their tendency to store more myosin motors and molecules needed for contraction. Another effect was derived from the model of the contractile elastic disc mentioned earlier. From 
the dependency of the force on the spread area reported here and the model, we determined the development of the maximal force in dependency of the substrate stiffness, cell height and the adhesion layer characteristics. Given the very limited thickness of about $100 \mathrm{~nm}$ of platelets, we demonstrated that a saturation in force is expected for a substrate stiffness of well below $10 \mathrm{kPa}$. Changing the adhesion layer characteristics did not influence the force noticeably, indicating that in this regime, the height of the cell dominates the force development. Thus, we demonstrated that the mechano-sensitivity of platelets in terms of total force development is lacking within our stiffness regime due to their thin size.

Let us continue on the spatial distribution of the traction forces. Similar to other cells $[3,46]$, we observed that the forces from the platelets are transmitted near the periphery. This is in accordance with the previous study conducted by Schwarz Henriques et al. [107]. In particular, we found that most platelets exhibited two to four force spots. We have previously established that the contractile force within the platelet is maintained by myosin bound to filamentous actin. Moreover, the platelets are bound to the substrate. Hence, we assume that the force pattern we observed are due to the internal structure of the acto-myosin network and its direct connection to the substrate. In fact, it is known that the actin cytoskeleton exhibits different structures on flat substrates, especially of the thicker actin bundles $[48,90]$. These bundles come in shapes of pointy ellipsoids, triangles or rings. Most recently, a tension sensor binding to the integrin was developed and it could be shown that the high contraction force within a platelet is transferred at two to three spots at the outline of the platelet [125]. The force transmitting network within a platelet can then be simplified to structures as seen in Fig. 8.1. Thus, we argue that the observed force patterns correspond very well with the literature and is a direct result from the internal cytoskeletal structure. This is an interesting result when investigating the dipole ratio in dependency of the substrate elasticity. We reported that the dipole quotient, or the degree of isotropy, exhibited a slightly increasing trend for harder substrates. Connecting this to the cytoskeletal organisation, we deducted that the polarity increases moderately with the stiffness.

\subsection{Oscillatory Contractions}

We have now considered some general aspects we observed during the experiments. Let us now have a look at some features that are of special interest. In particular, let us discuss the oscillatory contraction behaviour more in detail. This includes 

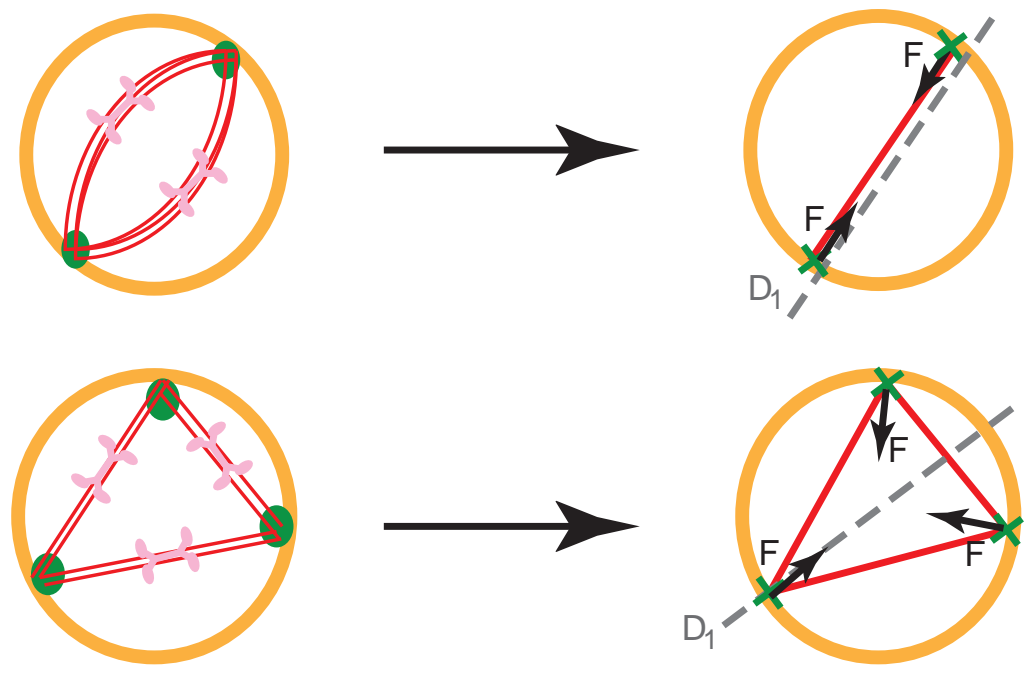

Figure 8.1.: Two examples for the simplification of the contractile system in the platelet. Inside the cell (orange), the actin filaments (red) form thicker actin bundles that may appear as pointy ellipsoids (upper part) or triangles (lower part). At the end of the bundles, the platelet is connected to the substrate by integrin clusters (green). The contraction is facilitated by the myosin motors (pink) bound to the actin. These networks can be reduced to forces starting at the integrin clusters pointing inwards towards the cell's centre. The actin bundles are reduced to a one-dimensional object for stabilisation and the force transmission through the cell. The expected major dipole axis is denoted by the dashed grey line. 
the study of the isotropy of the force patterns. Subsequently, we will take a closer look at the contraction under flow conditions, especially the orientation of the major dipole axis.

As shown in Sections 7.2.2 and 7.3.4, platelets could exhibit different contractile behaviour over time; they reached a force plateau, relaxed over time or started to oscillate. Combinations of these behaviours did occur. We have previously commented on the plateauing and relaxing contraction. Hence, we will now consider the most interesting behaviour of the three, the oscillations.

We observed in the previous chapter that the oscillation frequency of the platelets is, on average, around $13 \mathrm{mHz}$. For the static case, using a Fourier analysis, we demonstrated that the relative amplitude of the oscillations was generally higher than for the non-oscillatory case but no clear distinction was possible (Fig. 7.3 D). Instead, it seemed as though the transition between oscillating and non-oscillating was a gradual one. For the case of an external flow field, the oscillations reduced both in number of platelets exhibiting them and in the relative amplitude detected in Fourier space. Thus, we can first conclude that the mechanism that governs the appearance of oscillations is repressed by the flow.

In the previous section, we made two observations of the force development in platelets. The first was regarding the internal actin structure that transmits the force through the platelet. Second, we observed that previous work demonstrated that calcium is essential for the activation of the platelet and calcium flux across the membrane has been detected. In this work, we indirectly studied the first aspect, the internal actin structure as a possible reason of the oscillations. We noted previously that the force patterns arose from the acto-myosin network within the platelet. Hence, if the internal structure is a reason for the contractile behaviour, this would translate into the isotropy or anisotropy of the force pattern. We thus investigated the force dipole quotient as a measure of the degree of anisotropy. However, as seen in Fig. 7.6 D, no significant difference in the ratio was detected. We thus conclude that the structure of the actin cytoskeleton is not the reason behind the oscillatory behaviour.

Let us consider the second point we made, the level of intracellular calcium. While it was previously shown that calcium oscillations exist and are important during adhesion, the corresponding experiments were conducted by using VWF. The oscillations resulting from the interaction of VWF, the glycoprotein Ib-V-IX and the integrin $\alpha \operatorname{Ib} \beta 3$ were measured with a frequency of about one order of magnitude higher than our oscillations [84]. However, we did not include VWF in our system but fibrinogen. Also, these oscillations were shown to be of importance to 
stabilise the adhesion. Instead, let us consider a study on fibroblasts. In Lembong et al. [63], they investigated the calcium oscillations in fibroblast cultures on PAA gels. Several interesting observations were made. On substrates of different stiffnesses, calcium oscillations occurred at a frequency of about $10 \mathrm{mHz}$, independent of the stiffness. Instead, the oscillations seemed to depend on the state of the cytoskeleton where stabilised actin networks resulted in less oscillating cells. Blocking the myosin motors yielded the same results. It was thus argued that oscillations in calcium are dependent on the stability of the actin network and play a role in the force generation. Using this knowledge, we deduct that not the actin structure itself but instead the stability of the network determines whether or not we observe oscillations. Similar to their results, we did not detect any dependency of the stiffness. Instead, the oscillations were reduced by the application of external stress due to flow. First, by adding an external stress to the cell, we argue that a more stable actin network is needed for the cell to withstand the applied force. Additionally, let us consider the fact that we did not add any external calcium in the buffers. Hence, all calcium intake done by the platelets was only possible if another platelet previously released the calcium. Due to the continued flow, free molecules were constantly washed away. Thus, the chances of repeated uptake of calcium were reduced. This explains the phenomena that we observed.

To be able to find a proof for our hypothesis, we need to be able to image the actin cytoskeleton and the calcium flux at high resolution. Imaging of the actin structure while simultaneously recording the forces were tried. Here, we chose to use the live actin-dye SiR-Actin [73] as it had been shown to work on platelets on glass [90]. However, due to the added scattering in the substrate, no clear images could be recorded to support our hypothesis.

Before continuing with the next aspect, let us consider another oscillation that was reported in the context of platelet spreading. In Ref. [108], it was observed using scanning ion conductance microscopy that a platelet being activated by thrombin exhibits dynamic changes in the plasma membrane. These wave-like changes in the plasma membrane were proven to be due to an interaction between dynein and the actin cytoskeleton. Interestingly, the velocity of the waves in the outer membrane also oscillated and was estimated to be in the same frequency spectrum as the oscillations measured in the force presented here. Note that contrary to the forces, the observations on the spreading dynamics were made on polystyrene culture dishes instead of our softer gels. By contrast, in Sandmann et al. [101], using PDMS substrates of an intermediate stiffness between the PAA gels and the culture dishes, such changes were not observed, instead reporting 
a rapidly stabilising platelet contour. The latter experiments were made under similar conditions as the static experiments reported here. While we detected some changes in the intensity of the labelled membrane, given our low contrast and resolution compared to Ref. [108], it was not possible to identify them as actual membrane waves or just slight changes in the intensity in the rapidly bleaching dye. Nor were these changes in intensity restricted to oscillating cells. To be able to definitely determine a possible correlation between the membrane waves and the oscillatory behaviour, a super-resolved TFM method needs to be employed. An interesting point to consider here is an observation made by Wang et al. [125] using the integrative tension sensors. During adhesion and spreading, they reported two different tension regimes. One occurred during the adhesion and earlier spreading period and was characterised by a lower tension and ring-like distribution. At a later time point, a higher tension at two to three discrete locations were observed during the latter stages of spreading and during stabilisation of the area. As the initial spreading is driven by actin polymerisation, one can assume that the first tension regime corresponds to this process. However, as we reported here, the contractile forces measured here start later than the spreading (Fig. $7.3 \mathrm{~A}$ ), the higher tension regime is probably due to the actomyosin interaction. Hence, the oscillations in the membrane contour solely due to actin polymerisation needs to be decoupled from the oscillations of the contractile force.

\subsection{Force Orientation Within Flow Field}

While platelets did not adapt to the stiffness of the surrounding apart from a slight increase in anisotropy, under shear flow, an adaptation in contraction orientation was observed. As soon as the forces started to develop, the angle between the major dipole axis and flow direction stayed roughly the same over the remaining recording time. Hence, we concluded that the time interval determining the orientation of the cell in terms of the contractile force is the time between attachment and force development as already suggested by Myers et al. [81].

Our simulation of the fluid shear stresses during attachment demonstrated that for the highest flow rate, a maximal shear stress of $0.12 \mathrm{~Pa}$ was observed on top of the platelet and a maximal shear stress at the surface of the substrate of about $0.04 \mathrm{~Pa}$. Simultaneously, if we study the von Mises stresses experienced by the elastic, un-spread platelet, we observed a higher stress of over $0.3 \mathrm{~Pa}$ at the area of attachment at the front of the cell. Hence, from a material analysis point of view, at this position, the detachment was most likely to occur. Note that the actual 
deformation of the platelet was in the order of picometers. The stress distributions and deformations of the attached cell remained similar over all shear rates, only scaling according to the flow velocity. Interestingly, however, the angle between dipole axis and flow direction did change. For the lower shear rates, the angle averaged around $45^{\circ}$ while at the highest flow rate, the angle was close to $90^{\circ}$.

Let us first take a closer look at the analysis itself. From Chapter 3.2, we know that the dipole is the integral over the product of the distance and traction force. The major dipole axis then describes the axis of preferred contraction in a purely contractile cell. We have previously argued that the force patterns we observed were due to the structure of the thicker actin bundles anchored to the substrate, building structures of ellipsoids, triangles or circles. If we now only consider the major dipole axis to model the direction of contraction, we reduce the two-dimensional contraction to the problem a uni-axial contraction which is characteristic to an ellipsoidal actin network as sketched in Fig. 8.1, upper part. This is of course a simplification of the actual situation but to understand our data, let us continue with this model. The reduction in dimensionality for a triangular actin network is included in Fig. 8.1 by the dashed line of the major dipole axis for illustration. As we only expect angle values from $0^{\circ}$ to $90^{\circ}$ (compare Section 6.2.4), these two values mark our limiting cases. The corresponding actin models are depicted in Fig. 8.2, including the direction of the flow. Consider first the case of $0^{\circ}$ (Fig. 8.2 A). Here, one of the main anchor points, i.e. the points that transmit the force signal, is located at the place of highest von Mises stress (dark red arc). However, as we noted before, this is the point where our material, here our cell, first yields if the stress becomes too high. Taking our simplified platelet structure, this would be the point where the integrins would first break. While the force we exposed the platelet to was distinctly less than the measured detachment force of the $\alpha \operatorname{IIb} \beta 3$ integrin from fibrinogen $(<1 \mathrm{pN}$ vs $60 \mathrm{pN}$ to $100 \mathrm{pN}$ measured by Litvinov et al. [67]), it is still a critical point. For the case of a $90^{\circ}$ orientation (Fig. 8.2 B), the integrin clusters are found at places close to the lowest von Mises stress (dark blue arcs). Hence, the second configuration is considerably more stable in terms of withstanding an external applied force. In conclusion, from a mechanical point of view, a perpendicular alignment to the flow is the most reasonable.

This raises the question whether this alignment to the flow direction has been investigated and reported before. While only few works exist that study the force orientation under flow conditions, this has proven to be an interesting point in the process of angiogenesis [7], i.e. the formation of new blood vessels. Like the attachment of blood platelets, this phenomenon occurs during blood flow. Hence, 
A

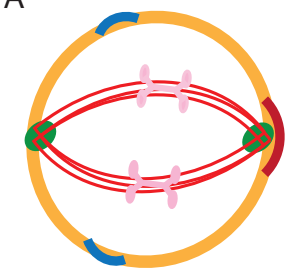

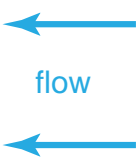

B

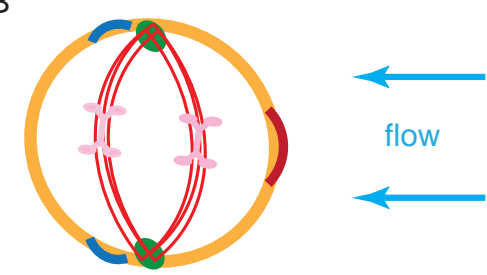

Figure 8.2.: The limits of orientation of the actin network (red) compared to the flow (blue). Here, we assume a contraction along a line, thus an ellipse-like actin bundle structure (compare Fig. 8.1). In $\boldsymbol{A}$, the actin alignment is parallel to the flow direction, the force transmitting anchors pointing towards the flow and away of it. In $\boldsymbol{B}$, the alignment is perpendicular to the flow direction. The force is generated by the interaction between the actin filaments and the myosin motors (pink). The approximate positions of the highest and lowest von Mises stress are included by the dark red and dark blue arcs, respectively. The figure is adapted from Hanke et al. [37].

similar experiments were conducted using layers of already attached endothelial cells $[47,57,92]$. For different shear stresses ranging from $0.014 \mathrm{~Pa}$ to $2 \mathrm{~Pa}$ and different geometries, the preferred direction of contraction with respect to the flow was determined. While all mentioned works showed a large variance in the angle, a general tendency towards $90^{\circ}$ was observed, comparable to our results. The variance in angle suggests that this response is not an 'all-or-nothing' process but the cells adapt gradually to the shear stress.

The gradual adaptation to external stress is an interesting result. From the purely mechanical analysis as done in our simulations, this cannot be directly concluded. Let us instead consider a different kind of experiment. In Kaunas et al. [51], endothelial cells were cultured on elastic substrates which were afterwards stretched in a cyclic manner. They did show that the actin stress fibres oriented in a perpendicular direction to the stretch direction. Most importantly, the orientation was dependent on the magnitude of the substrate deformation such that a threshold had to be exceeded to align all actin fibres in a perpendicular direction. Below this threshold, larger variations in orientation were seen, indicating that the alignment is a gradual process. This corresponds very well with our results here. Additionally, the orientation of the contraction concerning stretching of the cell was previously predicted by Schwarz et al. [104] when theoretically investigating the energy and force dipoles in elastic layers of cells. Here, a stretching of the cell layer was determined to yield a force orientation perpendicular to the deformation. One could now argue that we in our set-up did not actually stretch our platelets. 
However, given that the platelets are firmly attached to the substrate and pressed backwards by the flow, effectively, the membrane is stretched, making it possible to still use this argument. 


\section{Summary and Conclusion}

The aim of this work was to study the contractile behaviour of platelets under different external conditions. In particular, we were interested in if and how the force exerted by the platelets changes when exposed to different elastic environments or flow conditions. The investigation was divided into four parts, namely

- the development of an analysis algorithm for the traction force data,

- the measurements of the contractile forces when changing the stiffness of the underlying substrate,

- the development of a microfluidic chamber to use for TFM and

- the measurement of the contractile forces under various flow conditions.

Here, we chose to use the technique of TFM to measure the spatio-temporal evolution of the contractile forces. We observed that platelets, although only small cell fragments, exert forces of the same order of magnitude as considerably larger endothelial cells. Simultaneously, these forces were highly dynamic in time. Due to the resulting large deformations within very confined areas, previously reported algorithms using time-resolved, non-differential PIV, PTV or optical flow methods did fail to perform. We thus developed a differential PIV algorithm that, while specifically tailored to our specific problem, is easily adaptable to be utilised on TFM data of various kind. In general, as long as the time interval between successive images is sufficiently small, the analysis is independent of the recording time and the recorded total forces. To evaluate the functionality of our algorithm, we adapted an optical flow method similarly as the PIV approach to be able to analyse the same data. After calibrating both algorithms to each other, we demonstrated that both approaches yield the same total force for the data of contractile platelets. For the subsequent evaluation, the PIV algorithm was used as we proved that the need for regularisation is not present in this case, contrary to the optical flow method.

For the experiments including fluid flow, we first designed a microfluidic chamber that on one hand allowed us to mimic physiological conditions while simultaneously measure the traction forces of the platelets. Here, we developed a chamber design

and manufacturing routine that fulfilled these criteria as well as produced near 
identical, leak-tight copies of the device. Our chamber recreated an environment similar to that found in larger veins in terms of the shear rate. Within the chamber, a PAA substrate was embedded for the force measurements. We demonstrated that the flow velocities calculated from simulations fit very well with the real observed velocities of the fluid flow within the device. Hence, the device is usable even for different flow velocities to mimic situations in other blood vessels than used here. All components were fabricated in-house, allowing for changes in the geometries without varying the manufacturing protocol. For the better comparison of the static and flow experiments, we kept the chemical set-up of the fluids consistent between them. To this end, we included an external mixing device to combine the platelets and thrombin before the actual measuring chamber. The reaction time, i.e. the time between complete mixing of the fluids and them entering the chamber, was controlled by the length of their connecting tube as well as the flow rate of the thrombin solutions. An external mixing device had the additional advantage that the experiment can be adapted to also introduce other substances into the system in a controlled fashion. In conclusion, the developed device system was shown to be reliable to construct, well characterisable and highly variable if one intends to study the influence of different molecules on the platelet contraction.

During the experiments, we observed that the platelets contracted along their periphery in a discrete pattern with generally two to four maxima in force. This corresponds well with the previously reported actin structures within a platelet that often show geometries of pointy ellipsoids, triangles or more roundish shapes $[48,90]$. Simultaneously, it was shown by Wang et al. [125] that the force transmission occurs at two to three distinct places near the platelet's periphery. To bring these observations together and to demonstrate a dependency of the spatial force distributions on the actin cytoskeleton and following the force transmission, the development of the actin network during force exertion needs to be investigated. Here, a live actin stain could be combined with the used TFM protocols. Special care has to be taken here for the imaging of the actin as scattering within the substrate reduces the image quality.

Further, it was noted that the total force was independent of the external conditions but dependent on the size of the platelet. As such, the stiffness of the substrates as well as the shear rate does not influence the total force in the studied range. For the dependency of the contraction of the substrate elasticity, we determined from our data that stiffnesses of well below $10 \mathrm{kPa}$ are needed to observe an effect due to small thickness of the platelets. To test this theory, softer substrates have to considered under equal conditions. As we pointed out before, the deforma- 
tions in softer gels meant that beads were pressed out of focus during recording. Hence, to further the understanding of the mechano-sensitivity of platelets, the imaging technique first has to be adjusted to become three-dimensional, e.g. to the usage of a confocal microscope. Note that such a change in the imaging also influences the light exposure, time interval between images and the analysis algorithm. Alternatively, we also argued that the concentrations of fibrinogen and thrombin overrides any mechano-sensitive behaviour. Here, we used a comparatively high fibrinogen concentration where it was previously shown that area effects are diminished [93]. Moreover, we chose a thrombin concentration where both actin dynamics as well as forces are suspected to have reached their maximum $[65,108]$. It is thus of interest to change the concentrations of both substances to lower values to possibly regain the mechano-sensitivity if this was the cause. Here, one has to consider the influence of both the individual molecules as well as the combined effect of both.

As mentioned above, we also did not observe any dependency on the shear rate. This was either be due to a lack in sensitivity, an over-stimulation by the trigger molecules or due to the comparatively low shear rates tested here. In the human body, shear rates of much higher values are found which platelets have to withstand. While at much higher shear rates, the interaction between fibrinogen and the integrin $\alpha \mathrm{II} \beta \mathrm{b} 3$ does not drive the adhesion, they are still the driving reaction at shear rates of $100 \mathrm{~s}^{-1}$ or slightly above. Thus, it would be interesting to also study the contraction at higher shear stresses than used here. Before that, one first has to establish why a pure platelet solution did not show any adhesion at shear rates of about $50 \mathrm{~s}^{-1}$. As it was previously shown that adhesion can be facilitated when taking whole blood $[18,34,103]$, it could be tested whether the usage of whole blood yields different results in our device. Alternatively, as the adhesion of platelets in the human body is driven by red blood cells $[1,118]$, an alternative approach is the introduction of beads into the system to mimic the effect of the red blood cells.

Apart from the very strong contractile behaviour, we also observed that platelets exhibited three different contractile behaviours: a force plateau, relaxation after initial contraction as well as oscillations. We argued previously that the behaviour is dependent on the amount of chemical components present at the side of adhesion as well as inside the internal storages of the platelets. One important point we made was that with the current set-up, platelet contraction was maintained by relying on the molecular storages of the cells themselves. Adding molecules such as calcium or ATP into the buffer may thus dramatically change the behaviours 
observed here. In particular, as we argued that the relaxation seen was due to depletion of contraction triggering substances, an addition of said molecules would result in a reduction of relaxing cells. Instead of changing the buffer itself, the inclusion of these molecules in a controlled fashion is possible by using our mixing device, thus actively influencing the platelets' contractile behaviour. At the same time, the imaging of the flux of calcium during the contraction may answer the question whether this causes the oscillations in contraction. The latter experiment needs to be conducted either at static conditions or lower flow rates than used here as the oscillations were repressed during our flow measurements.

Lastly, we reported that the orientation of the major dipole axis to the flow direction changed with increasing shear rate. For lower fluid shear stress, on average, the angle was about $45^{\circ}$ while it increased to nearly $90^{\circ}$ at the highest shear stress. We argued that this corresponds to a gradual adaptation to the external force field and a perpendicular orientation of the actin cytoskeleton is more stable from a mechanical point of view. To confirm this hypothesis, both higher as well as lower shear rates have to be studied. For lower shear rates, the angle is than predicted to become more evenly distributed between $0 \circ$ and $90 \circ$ as expected under static conditions. Higher shear rates on the other hand would result in a clear preference of a perpendicular direction. As we build our argumentation on the assumption that the actin cytoskeleton followed the angle observed here, a live imaging of the actin network would further illustrate the effect of the flow on the contraction.

From this work, we conclude that platelets, small cell fragments lacking any transcription apparatus, are not mechano-sensitive to a wide range of tissues found in the human body, including muscle tissue. Instead, the magnitude of their response in terms of contractile force is governed by their size and exposure to trigger substances or adhesion proteins. On one hand, the size determines the amount of i.a. active proteins and ions contained within the cell. On the other hand, their height determines the stiffness range to which they are able to adapt their force to. In particular, this stiffness range is found to be well below $10 \mathrm{kPa}$, given sufficiently high chemical stimulus. Hence, a blood clot contracts consistently and largely independently of its position in the body. The crucial part for the contraction orientation is the external stress distribution around the platelet at the time point of attachment. This determines the internal orientation of the cell in terms of the force transmitting network in the sense that the stress acting on the network is reduced to an optimum. Thus, for small and thin blood platelets, the temporal 
contraction development is governed by the concentration of trigger substances and its size while its spatial force distribution is determined by the active external stress. 



\section{A. Appendix: Mathematical Proofs}

In this appendix, we provide some more details about mathematical derivations which have been omitted in the main text.

\section{A.1. Regularisation Using PIV and Lagrangian Markers}

In the experiments in Section 5.4.2.2, we observed that in the FTTC method using the Lagrangian marker approach, the regularisation parameter $\alpha$ could be set to zero without obtaining diverging approximations to the force field. At a first glance, this behaviour is counter-intuitive since the Boussinesq equation (5.1) is an ill-posed problem. In this section, we provide mathematical reasons why regularisation is not needed in this special case.

Earlier, we have shown that the eigenvalues of $\hat{\mathbf{G}}$ are given by $\beta(1-\nu)|\mathbf{k}|^{-1}$ and $\beta|\mathbf{k}|^{-1}$, where $\beta=\frac{2(1+\nu)}{E}$. For simplicity, let us only assume the case of $\lambda_{\mathbf{k}}=\beta|\mathbf{k}|^{-1}$. We now see that for each $\mathbf{k}$, the problem $T x=y$ reduces to

$$
\hat{x}_{k}=\lambda_{\mathbf{k}}^{-1} \hat{y}_{k}
$$

Additionally, we observed that the use of PIV and marker tracking results in a decay of the displacement in Fourier space as

$$
\left|\hat{y}_{\mathbf{k}}+\widehat{\delta y}_{\mathbf{k}}\right| \leq c_{y}|\mathbf{k}|^{-3}
$$

Here, this decay also includes the errors. So the a-priori assumption is that both $\hat{y}_{\mathbf{k}}$ and $\widehat{\delta y}_{\mathbf{k}}$ decay as $|\mathbf{k}|^{-3}$ individually. So can we now construct a space which includes all $\hat{y}_{\mathbf{k}}$ with their corresponding errors? One such space is

$$
\hat{Y}_{0}=\left\{\left.\hat{y} \in \hat{Y}\left|\sum_{k=-\infty}^{\infty} k^{2}\right| \hat{y}_{\mathbf{k}}\right|^{2}<\infty\right\}
$$


since all sequences $\hat{y}_{\mathbf{k}}$ with a decay like $|\mathbf{k}|^{-3}$ belong to this space. We then set $Y_{0}=\mathcal{F}^{-1}\left(\hat{Y}_{0}\right)$ where $\mathcal{F}^{-1}$ denotes the inverse Fourier transform. Further, we define a norm so that

$$
\|y\|_{Y_{0}}=\left(2 \pi \sum_{k=-\infty}^{\infty} k^{2}\left|\hat{y}_{\mathbf{k}}\right|^{2}\right)^{1 / 2}
$$

This subspace fulfils our requirements for a space containing our data. Formally, this can be sketched in the following diagram:

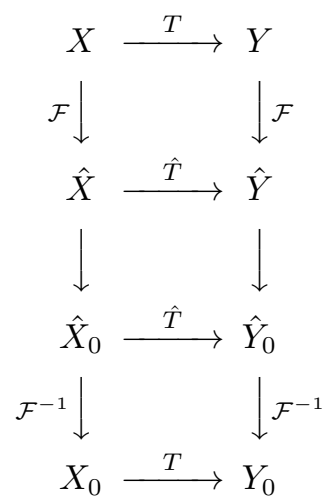

However, if we want to show that $T x=y$ is well-posed, we still need to show that $T$ is bounded in the norms of these spaces. We know that $\hat{x}=\lambda_{\mathbf{k}}^{-1} \hat{y}_{\mathbf{k}}$. Hence,

$$
\begin{aligned}
\|x\|^{2} & =2 \pi \sum_{k=-\infty}^{\infty}\left|\hat{x}_{\mathbf{k}}\right|^{2}=2 \pi \sum_{k=-\infty}^{\infty}\left|\lambda_{\mathbf{k}}\right|^{-2}\left|\hat{y}_{\mathbf{k}}\right|^{2} \\
& \leq \gamma^{2} 2 \pi \sum_{k=-\infty}^{\infty} k^{2}\left|\hat{y}_{\mathbf{k}}\right|^{2} \leq \gamma^{2}\|\hat{y}\|_{\hat{Y}_{0}}
\end{aligned}
$$

where $\gamma=1 /(\beta(1-\nu))$. Thus, it follows that

$$
\left\|\hat{T}_{0}^{-1}\right\| \leq \gamma
$$

Hence, the operator is bounded within our sub-space, both in Fourier space as well as in real space. 
For our specific application, we will use the discrete Fourier transform. This means that the infinite amount of modes $\mathbf{k}$ will be reduced to a finite number corresponding to the amount of finite grid-points $N$ used in the transform. However, the derivations above will still hold true. In particular, the operator $\hat{T}_{0, N}$ will still be bound by $\gamma$ which is independent of $N$.

Conclusion. Using the a-priori information of the Fourier coefficients, we can construct a sub-space containing all our data such that the operator $T$ becomes bounded thus continuous by a given constant. This means that our problem becomes well-posed. This also holds true in the discrete case.

\section{A.2. An Error Estimation for the Dipole Quotient Averaging}

As we only consider absolute values of the eigenvalues, we can assume that $0<$ $\lambda_{\min }(\mathbf{A}) \leq \lambda_{\max }(\mathbf{A})$. Can we find a relationship between the norms of the matrices and the corresponding eigenvalues to determine an estimation of $\delta$ ? We have the the following perturbation theorem for eigenvalues (Ref. [9], Theorem 6.9.7).

Theorem. Assume $\mathbf{B}$ is a normal matrix and $\mathbf{C}$ is any matrix. Then, for any given eigenvalue $\lambda(\mathbf{C})$ there is an eigenvalue $\lambda(\mathbf{B})$ such that

$$
|\lambda(\mathbf{B})-\lambda(\mathbf{C})| \leq\|\mathbf{B}-\mathbf{C}\|_{2} .
$$

We apply this theorem with $\mathbf{B}=\mathbf{A}^{*}$ and $\mathbf{C}=\mathbf{A}$ as well as with the estimate Eq. (5.45). Hence, that if $\lambda_{\min }(\mathbf{A}) \leq \epsilon=|\tilde{\mathbf{A}}|$, then $\lambda_{\min }(\mathbf{A})-\epsilon \leq 0<\lambda_{\min }(\mathbf{A})+\epsilon$. Furthermore, we see that $\lambda_{\min }(\mathbf{A})-\epsilon \leq \lambda_{\min }\left(\mathbf{A}^{*}\right) \leq \lambda_{\min }(\mathbf{A})+\epsilon$. It follows that $\lambda_{\min }\left(\mathbf{A}^{*}\right)$ can be arbitrarily small such that $q^{*}$ and hence $\delta$ will increase unboundedly. 
Let us now assume that $\epsilon<\lambda_{\min }$. Then there exist $\mu$ and $\nu$ with $|\mu|,|\nu| \leq \epsilon$ such that $\lambda_{\max }\left(\mathbf{A}^{*}\right)=\lambda_{\max }(\mathbf{A})+\nu$ and $\lambda_{\min }\left(\mathbf{A}^{*}\right)=\lambda_{\min }(\mathbf{A})+\mu$. Then we can estimate $\delta$ by

$$
\begin{aligned}
\delta & =\left|q-q^{*}\right| \\
& =\left|\frac{\lambda_{\max }(\mathbf{A})}{\lambda_{\min }(\mathbf{A})}-\frac{\lambda_{\max }\left(\mathbf{A}^{*}\right)}{\lambda_{\min }\left(\mathbf{A}^{*}\right)}\right| \\
& =\left|\frac{\lambda_{\max }(\mathbf{A}) \lambda_{\min }\left(\mathbf{A}^{*}\right)-\lambda_{\max }\left(\mathbf{A}^{*}\right) \lambda_{\min }(\mathbf{A})}{\lambda_{\min }(\mathbf{A}) \lambda_{\min }\left(\mathbf{A}^{*}\right)}\right| \\
& =\left|\frac{\lambda_{\max }(\mathbf{A}) \cdot\left(\lambda_{\min }(\mathbf{A})+\mu\right)-\lambda_{\min }(\mathbf{A})\left(\lambda_{\max }(\mathbf{A})+\nu\right)}{\lambda_{\min }(\mathbf{A}) \cdot\left(\lambda_{\min }(\mathbf{A})+\mu\right)}\right| \\
& =\left\{-\mu \geq-\epsilon ;|\mu|,|\nu| \leq \epsilon ; 0<\lambda_{\min }(\mathbf{A}) \leq \lambda_{\max }(\mathbf{A})\right\} \\
& \leq \frac{\left|\mu \lambda_{\max }(\mathbf{A})\right|+\left|\nu \lambda_{\min }(\mathbf{A})\right|}{\lambda_{\min }(\mathbf{A})\left(\lambda_{\min }(\mathbf{A})+\mu\right)} \\
& \leq \frac{\epsilon\left(\lambda_{\max }(\mathbf{A})+\lambda_{\min }(\mathbf{A})\right)}{\lambda_{\min }(\mathbf{A})\left(\lambda_{\min }(\mathbf{A})-\epsilon\right)} \\
& :=\hat{\delta} .
\end{aligned}
$$




\section{B. Appendix: Matlab Programmes}

\section{B.1. Main Programme}

The programme Traction_Main.m denotes the main programme and calls all subfunctions for the analysis.

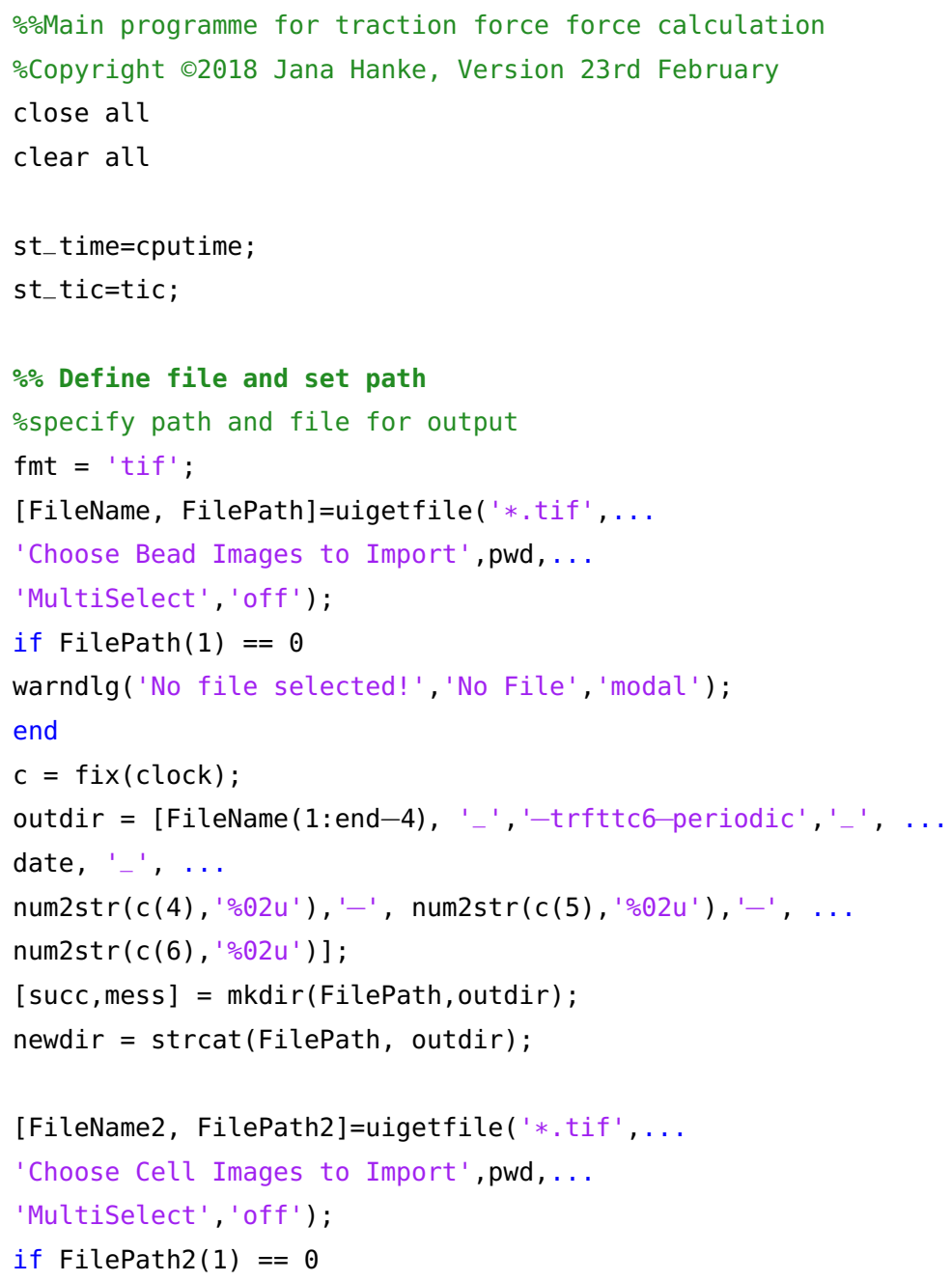




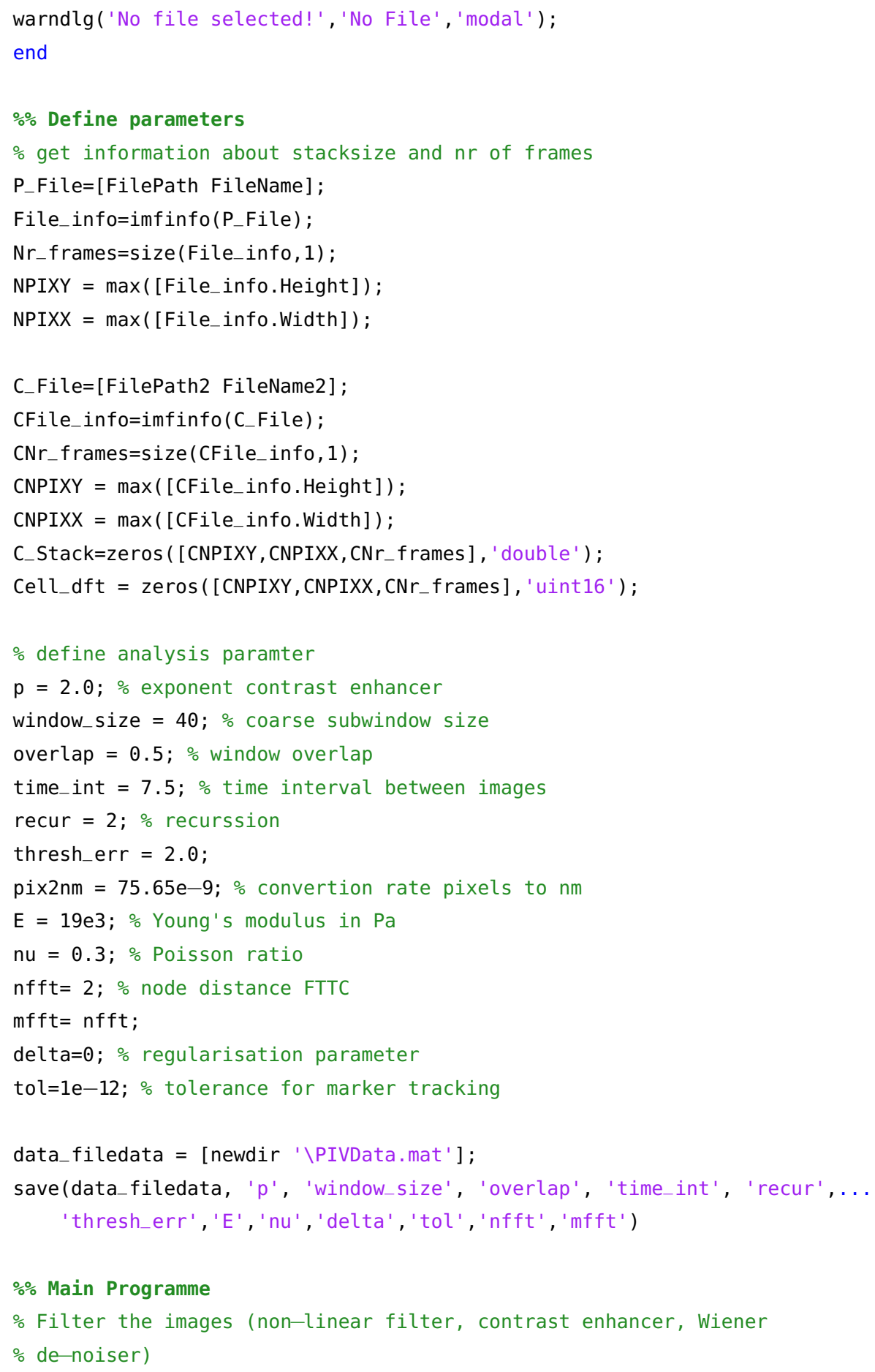




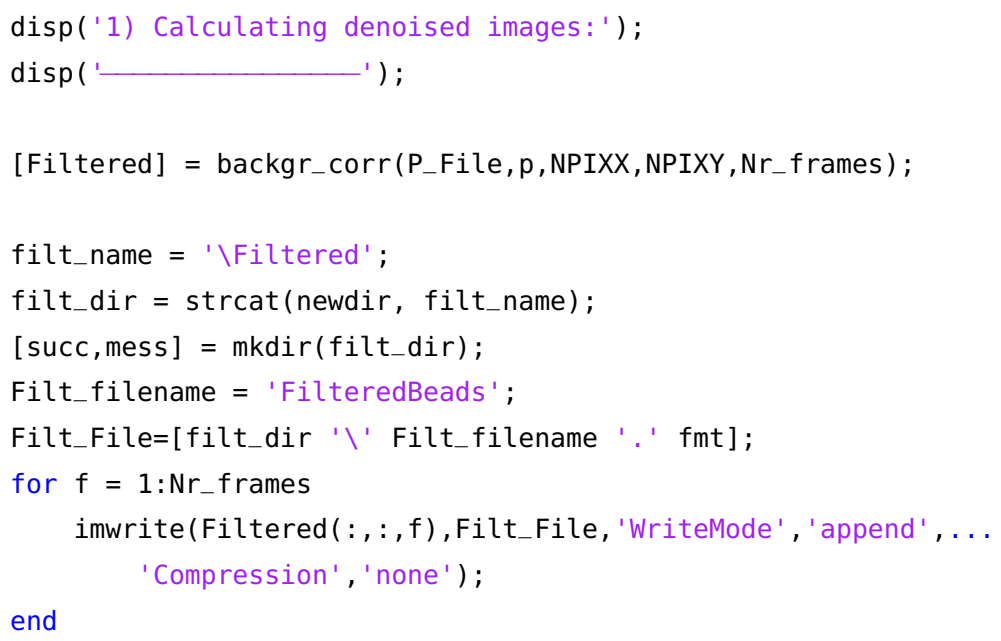




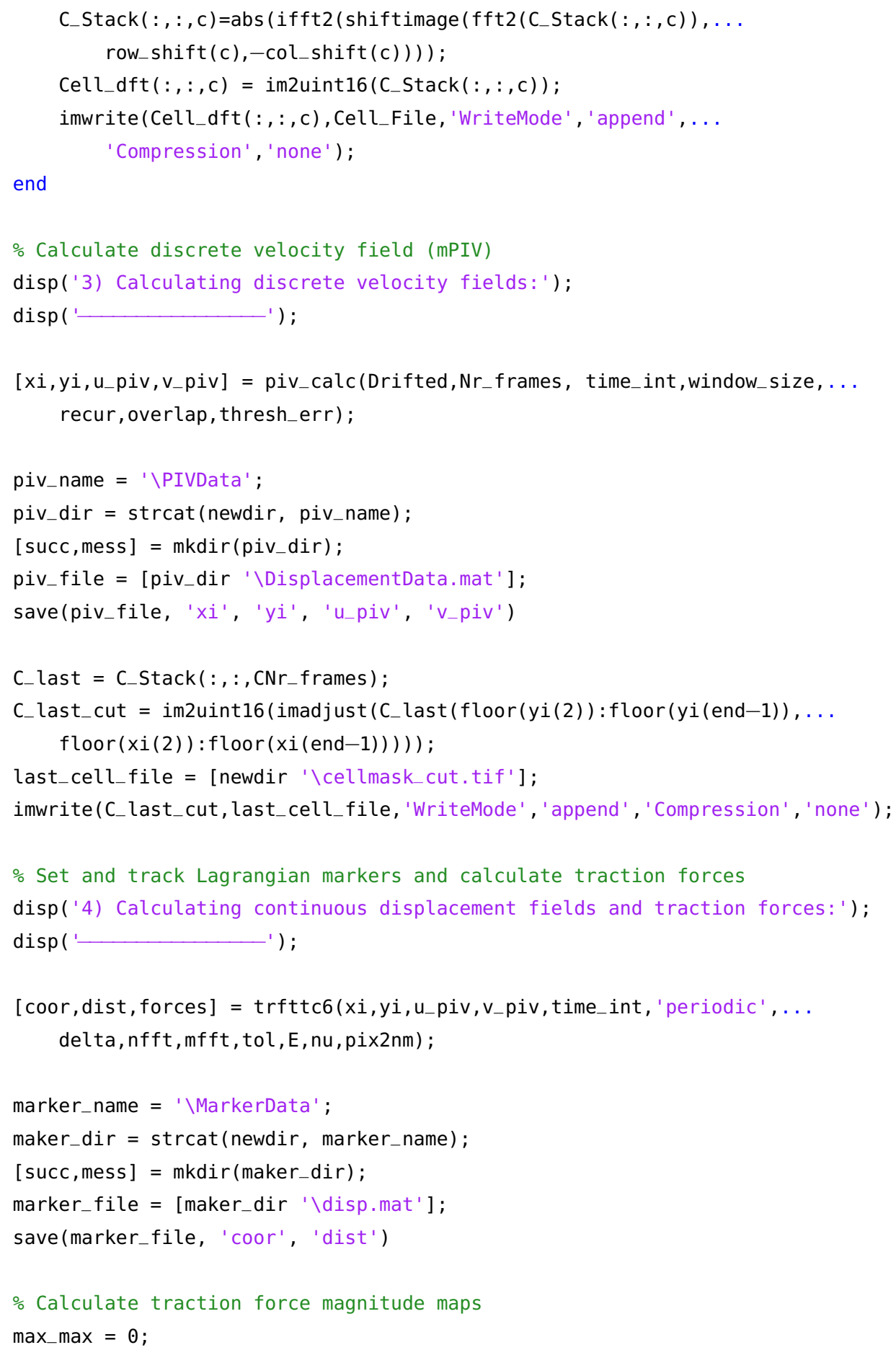




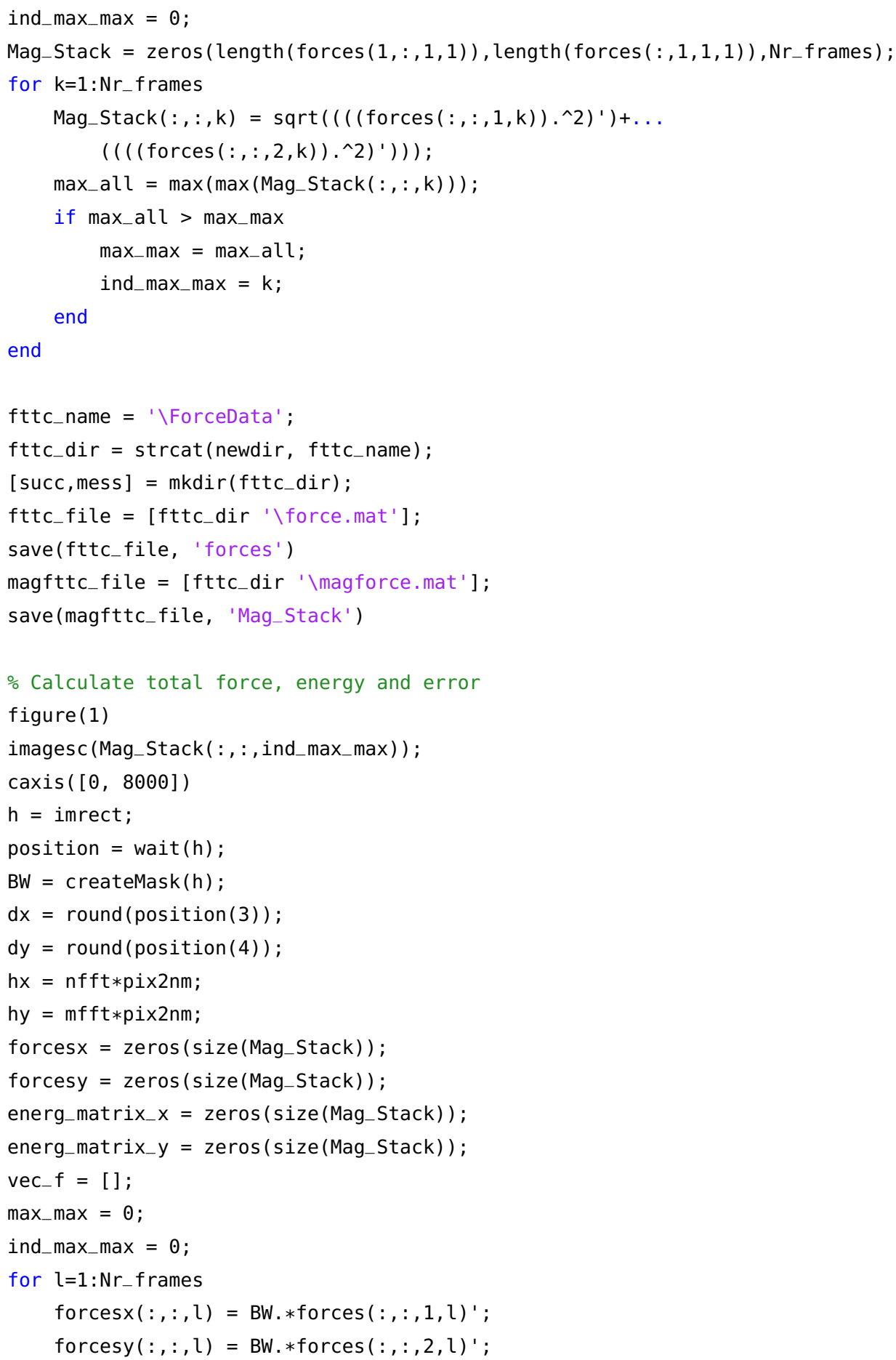




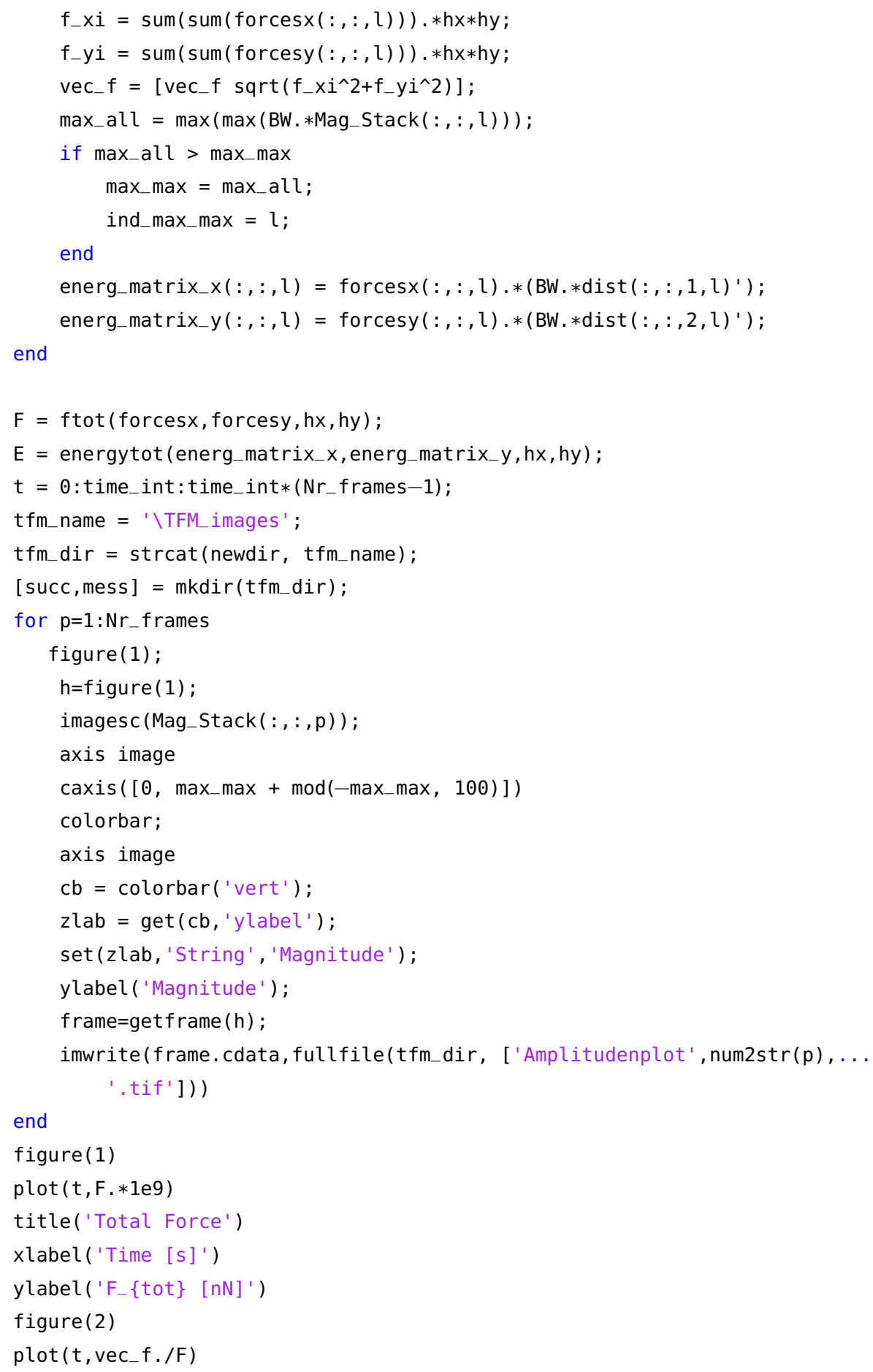




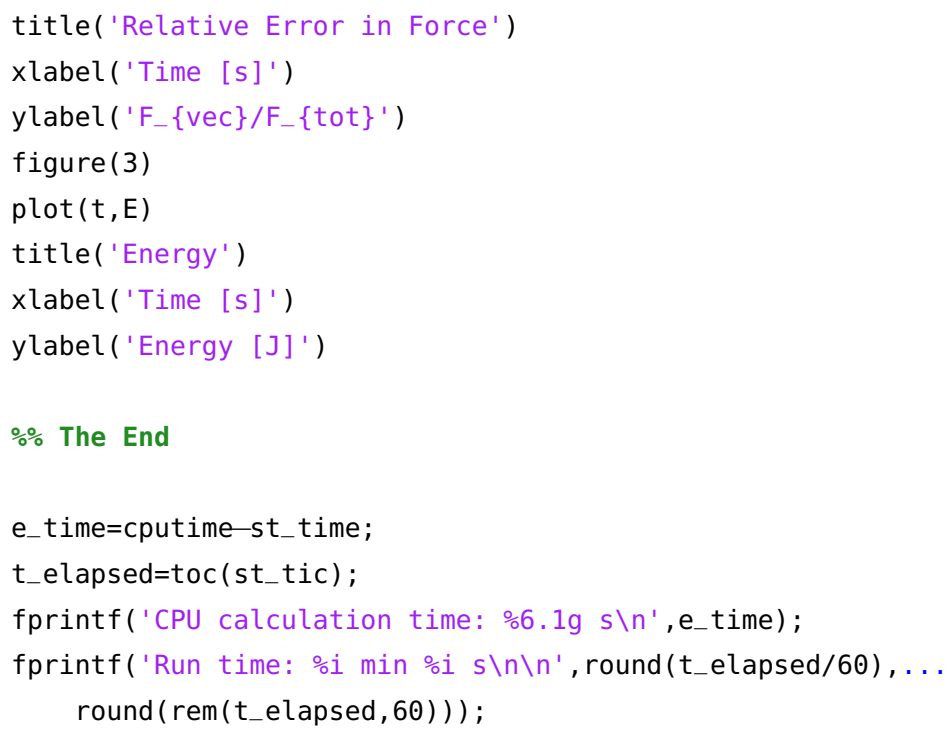

\section{B.2. Image Contrast Enhancer}

The programme backgr_corr.m increases the contrast in the recorded images as well as reduces the noise level.

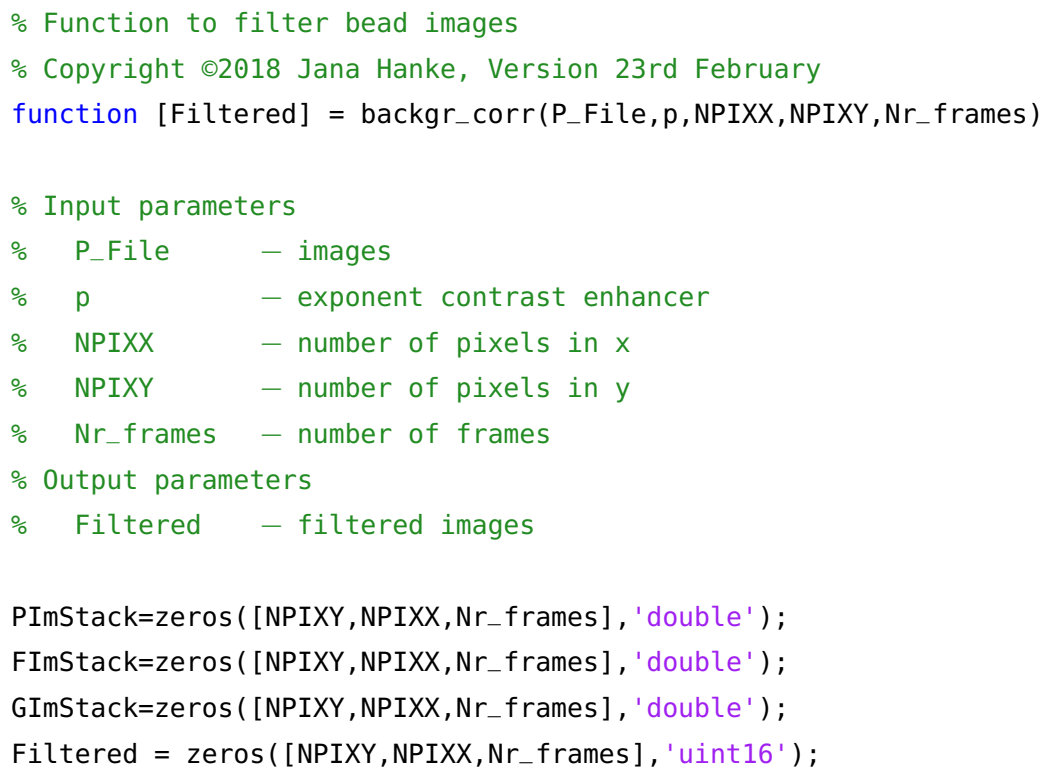


$\%$ Enhance contrast and de-noise

for $\mathrm{im}=1: \mathrm{Nr} r_{-}$frames

$\operatorname{PImStack}(:,:, i m)=\operatorname{im2double}\left(\operatorname{imread}\left(P_{-}\right.\right.$File, im $\left.)\right)$;

Im_Adjusted $=$ imadjust $(\operatorname{PImStack}(:,:$, im $))$;

$\operatorname{FImStack}(:,:$, im $)=\operatorname{Im} \_$Adjusted.^p;

$\operatorname{GImStack}(:,:, \operatorname{im})=\operatorname{wiener} 2(\operatorname{FImStack}(:,:, \operatorname{im}),[3$ 3] $)$;

Filtered $(:,:$, im $)=$ im2uint16 $(\operatorname{GImStack}(:,:$, im) $)$;

end

clear im

\section{B.3. Drift Correction}

drift_corr.m corrects the drift in the recording based on the filtered images.

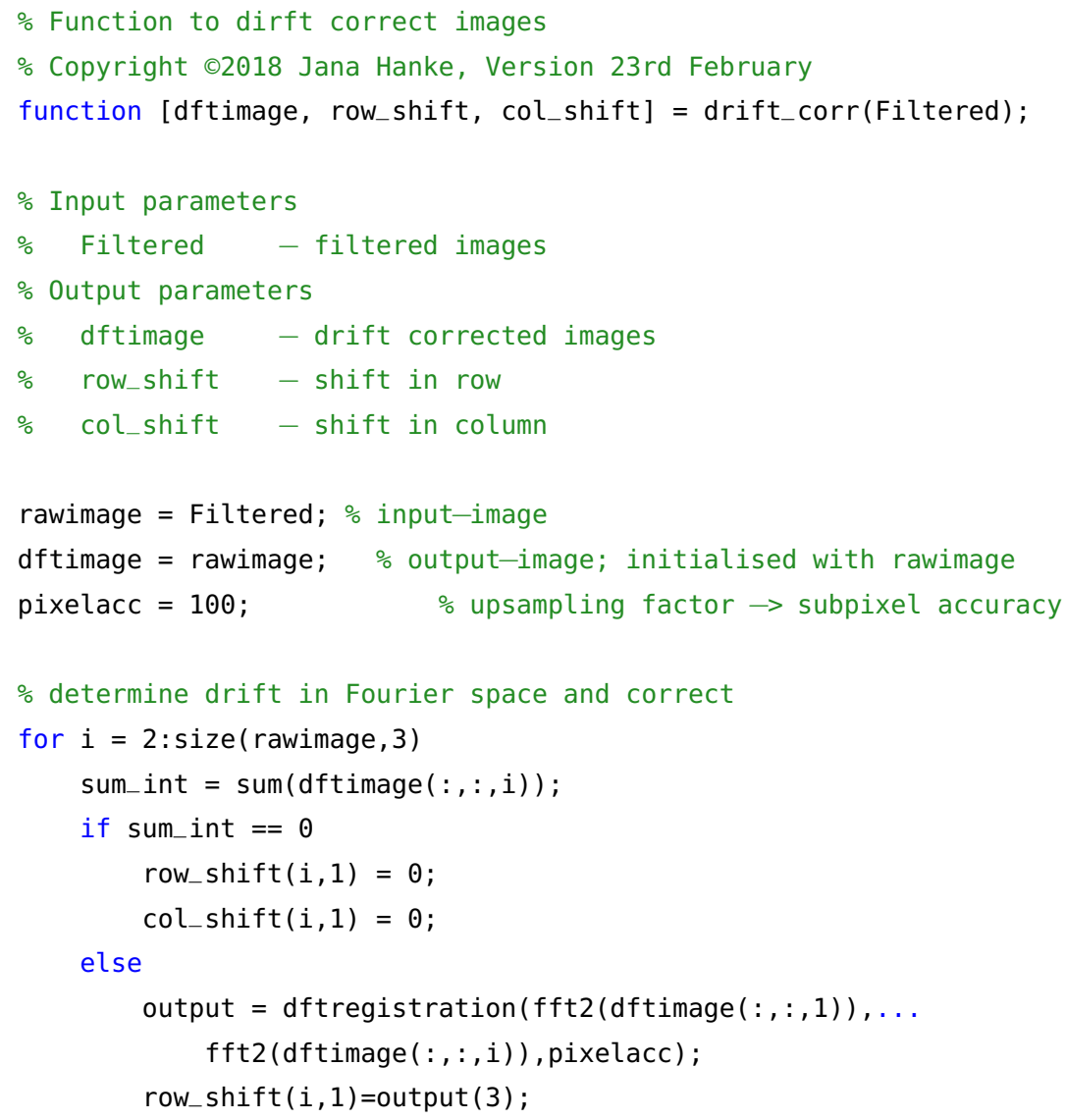




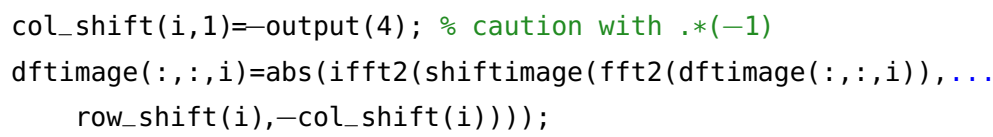

\section{B.4. Calculate Velocity Fields}

piv_calc.m determines the discrete velocity fields using PIV.

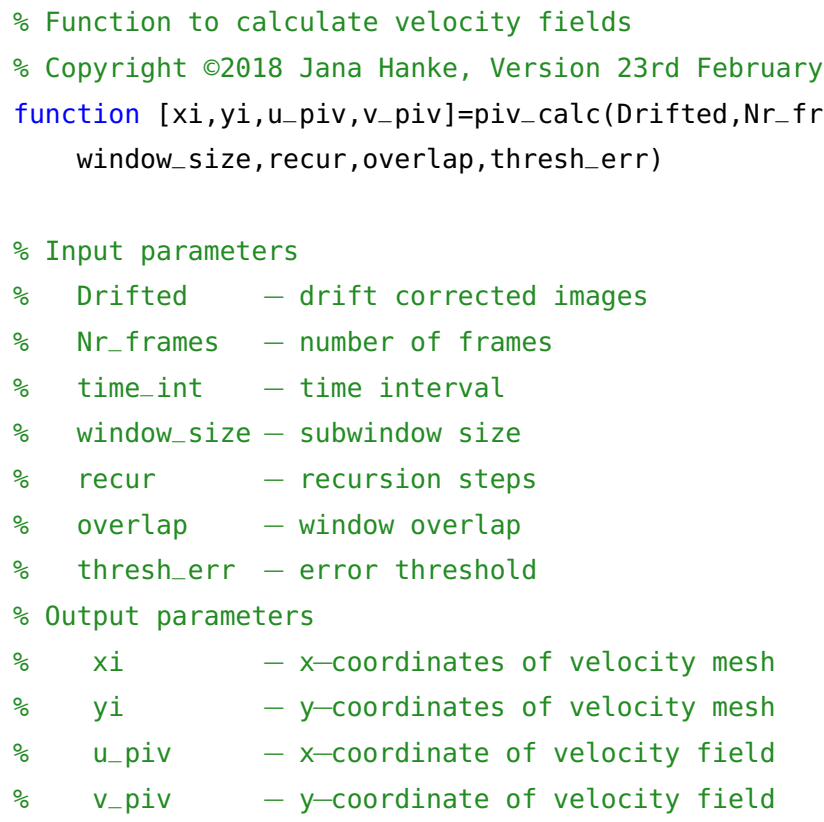




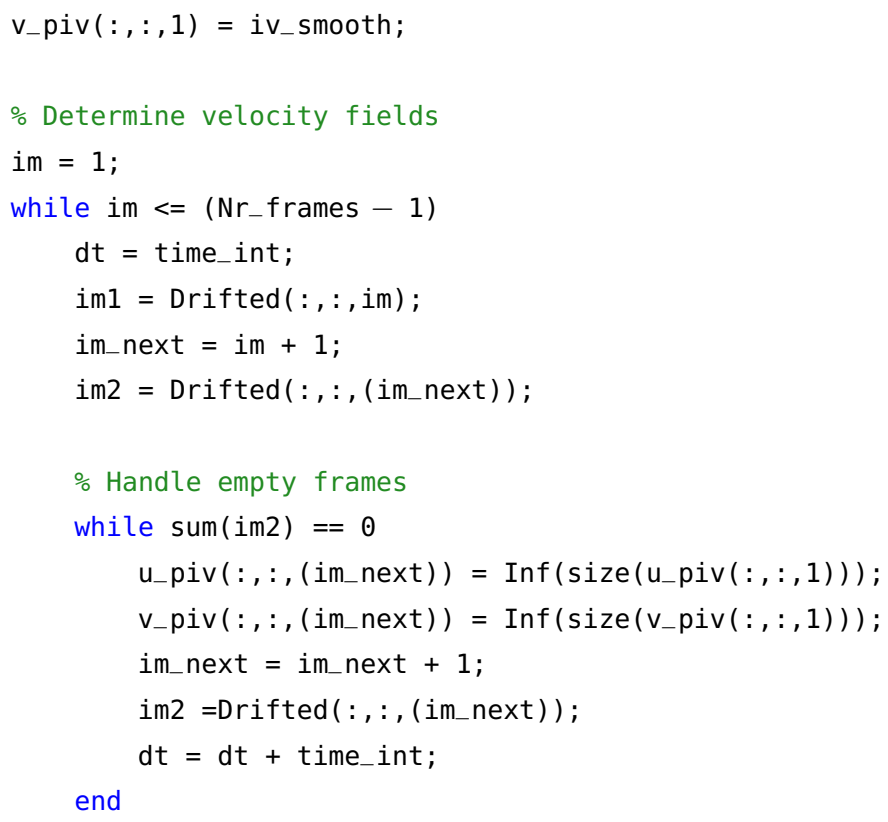

\% Compare forwards and backwards and average $\operatorname{disp}($ int2str(im))

[xi, yi, iul, iv1] = mpiv(im1, im2, window_size, window_size,... overlap, overlap, $0,0, \mathrm{dt},{ }^{\prime} \mathrm{cor}^{\prime}$, recur, 0$)$;

[iu_filt1, iv_filt1, iu_interp1, iv_interp1] = mpiv_filter(iul, iv1, 2, .

thresh_err, 3, 0);

[xi, yi, iu2, iv2] = mpiv(im2, im1, window_size, window_size,...

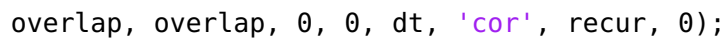

[iu_filt2, iv_filt2, iu_interp2, iv_interp2] = mpiv_filter(iu2, iv2, 2, thresh_err, 3, 0);

u_piv $(:,:($ im_next $))=0.5 . *\left(i u_{-}\right.$interp1-iu_interp2) ;

$v_{-} \operatorname{piv}\left(:,,_{\left(i m \_n e x t\right)}\right)=0.5 . *\left(i v_{-}\right.$interp1-iv_interp2);

im = im_next;

end

clear im 


\section{B.5. Calculate Displacements and Traction Forces}

The function trfttc6.m calculates both the displacements by Lagrangian marker tracking as well as the traction forces using FTTC. The sub-functions used for the FTTC part are given below, called initfttc.m and sfftc.m.

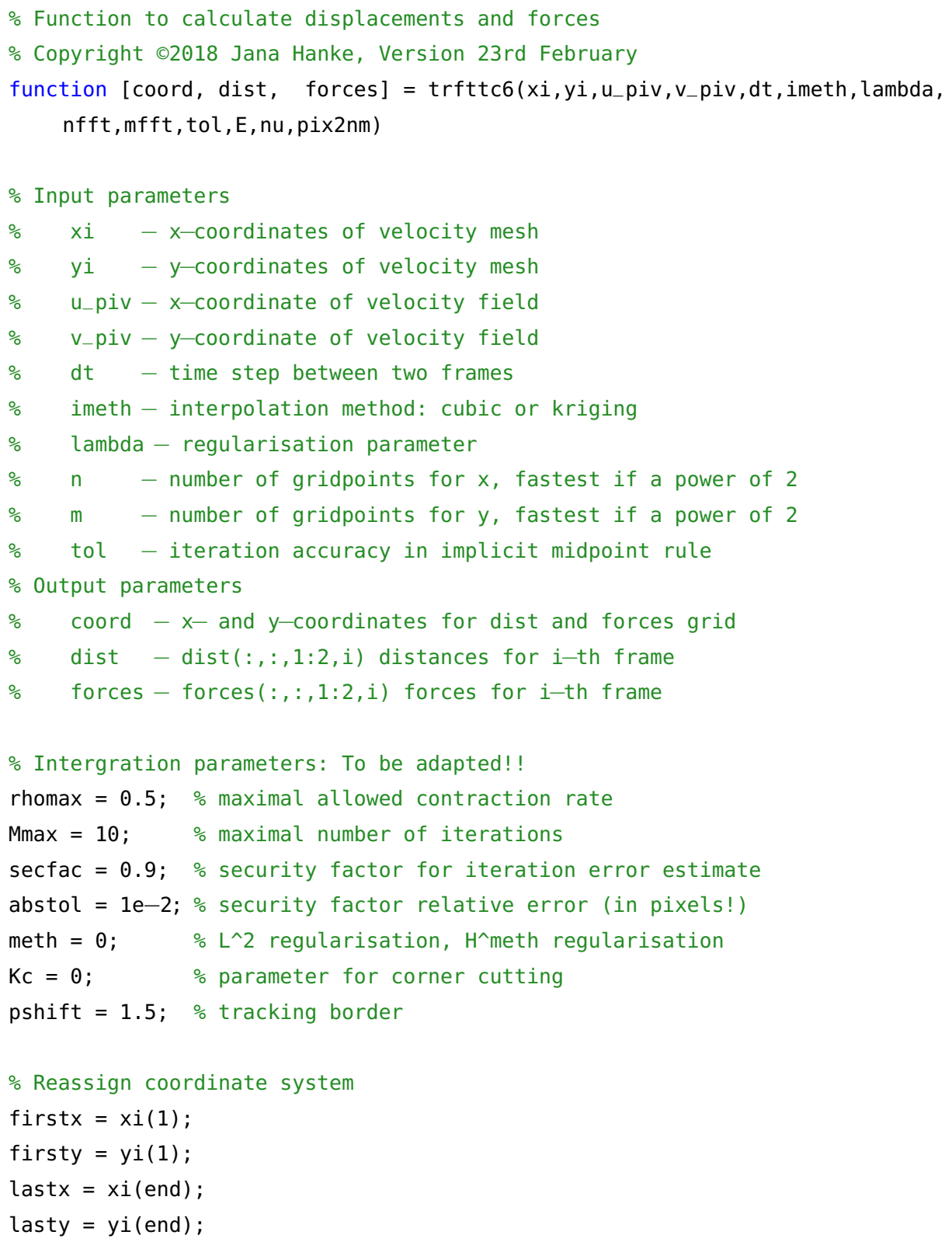




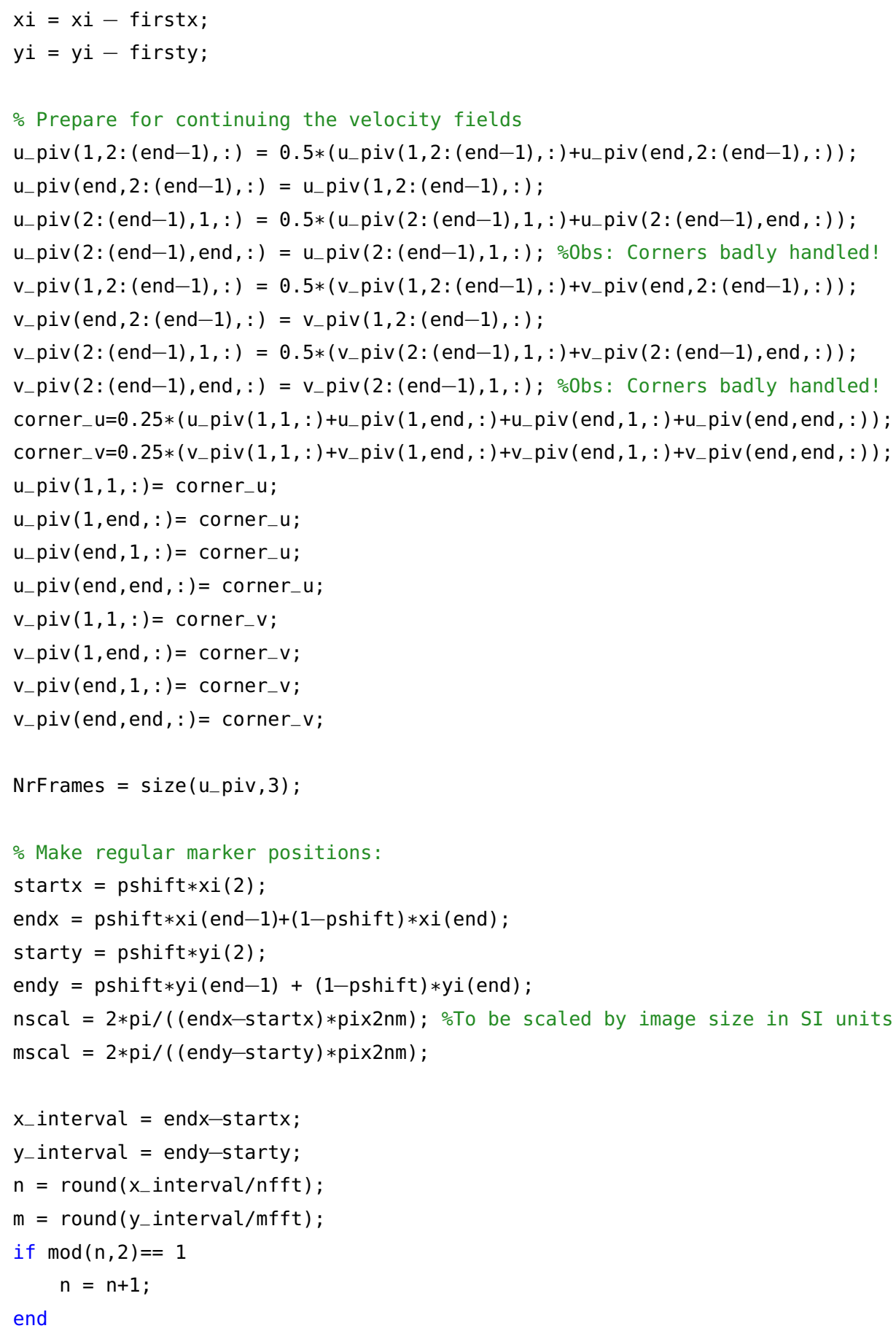




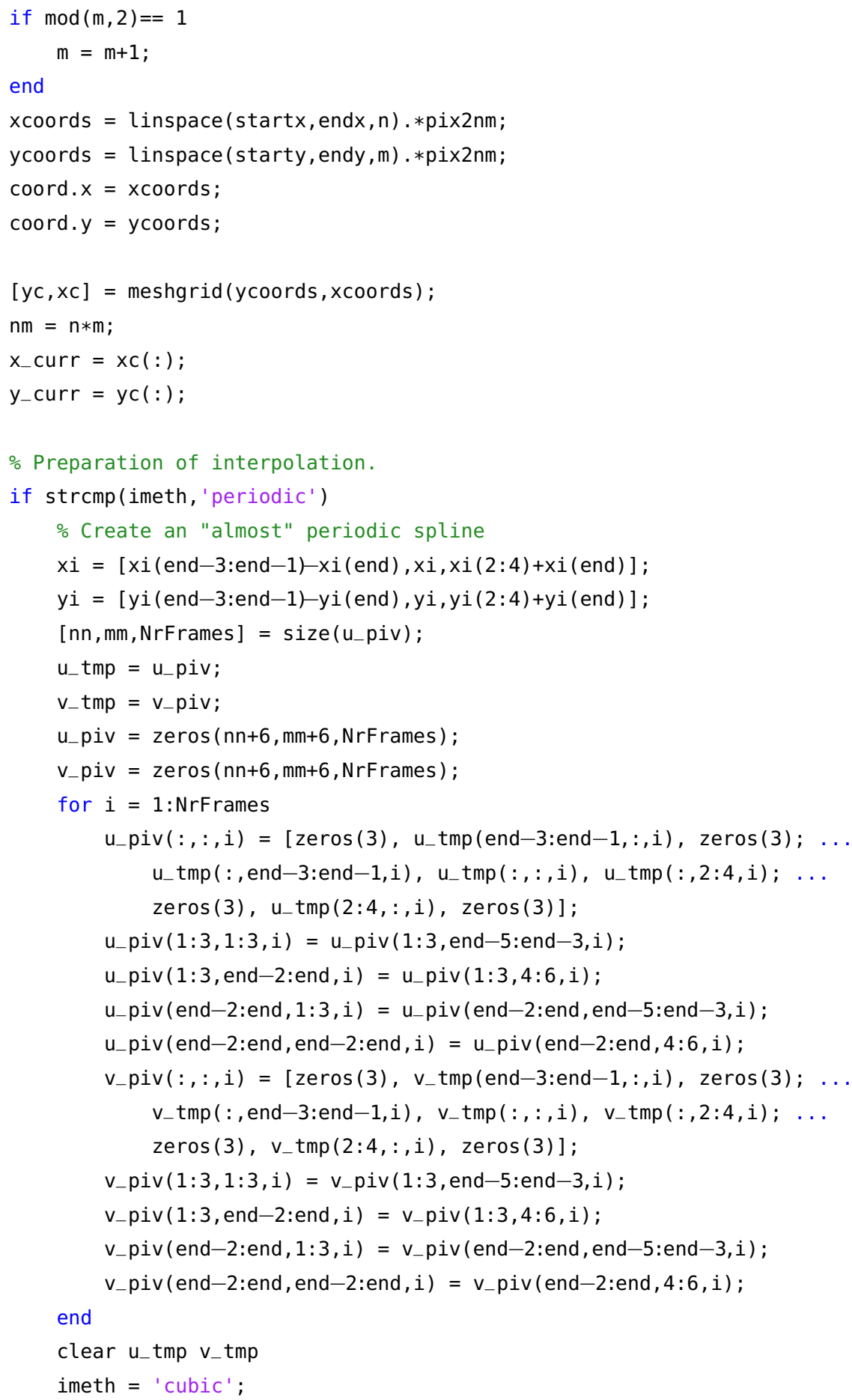




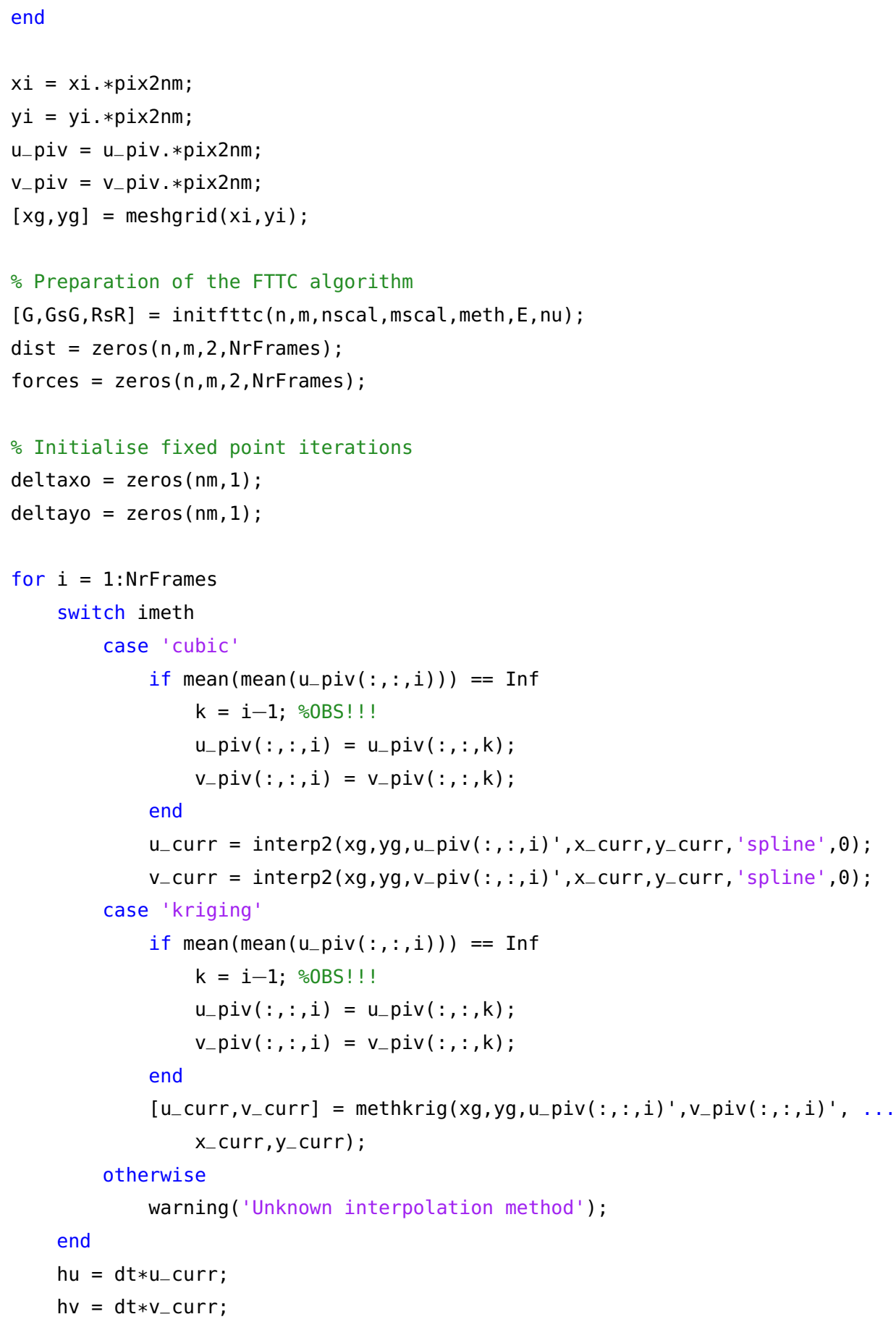




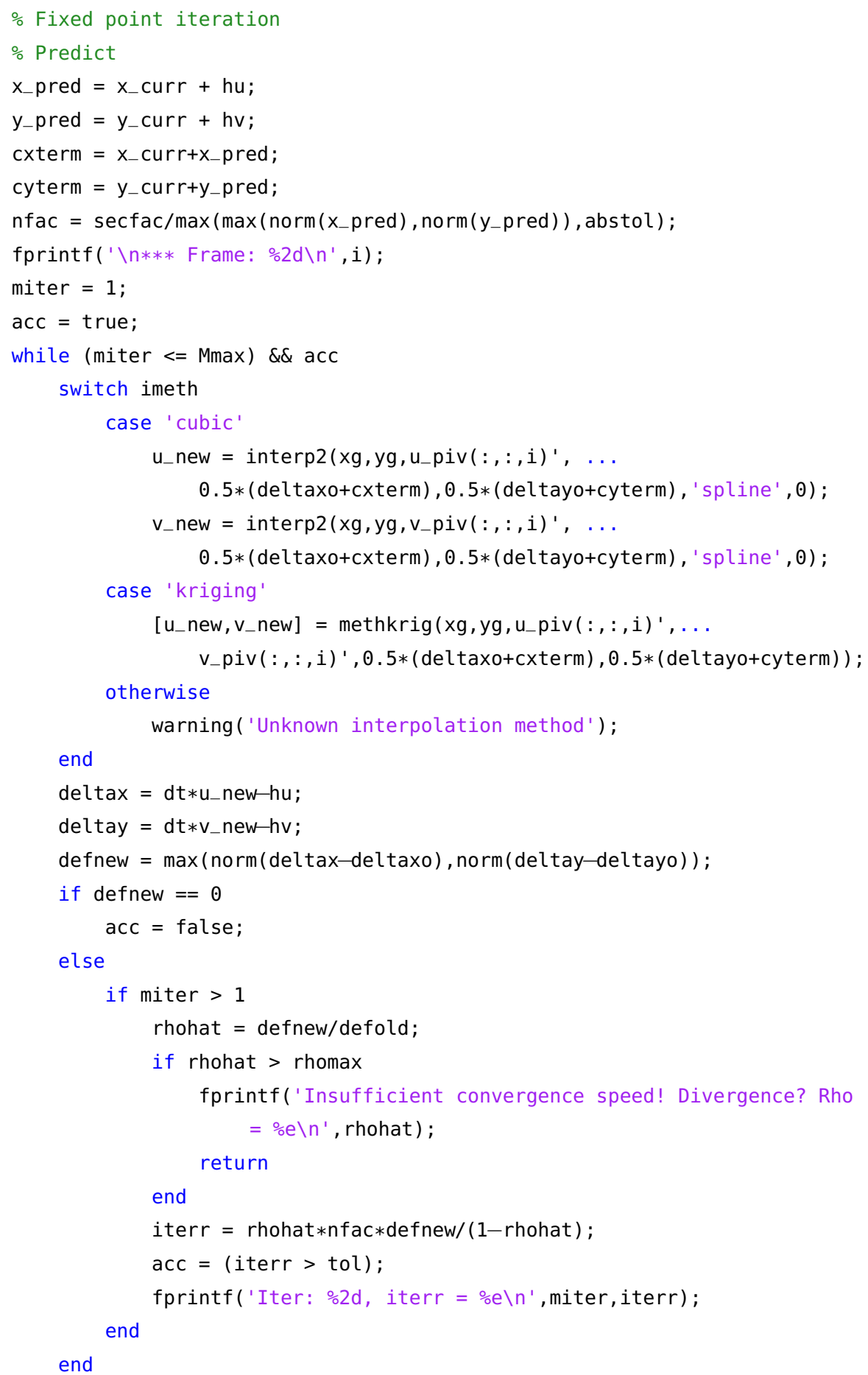




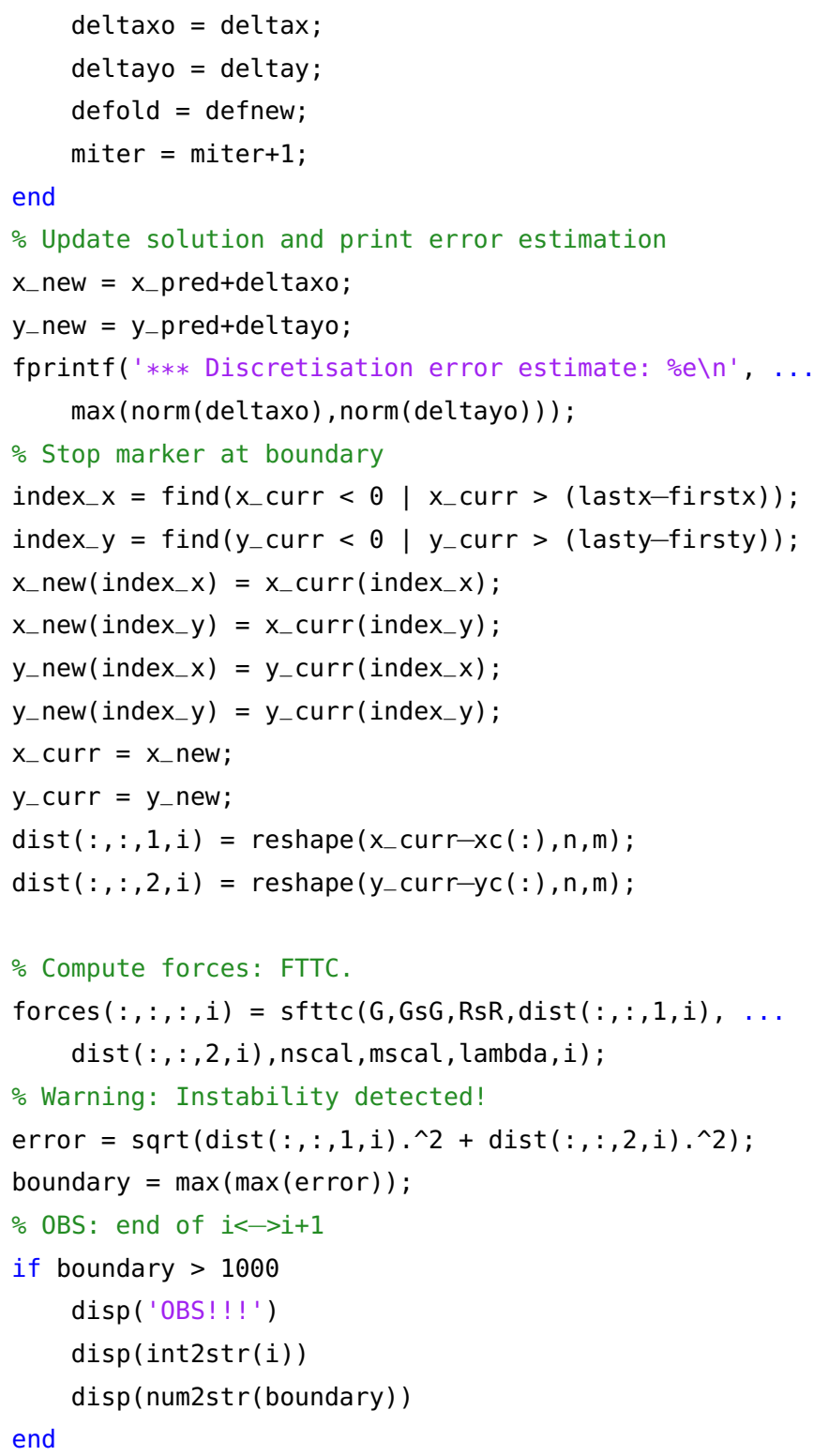




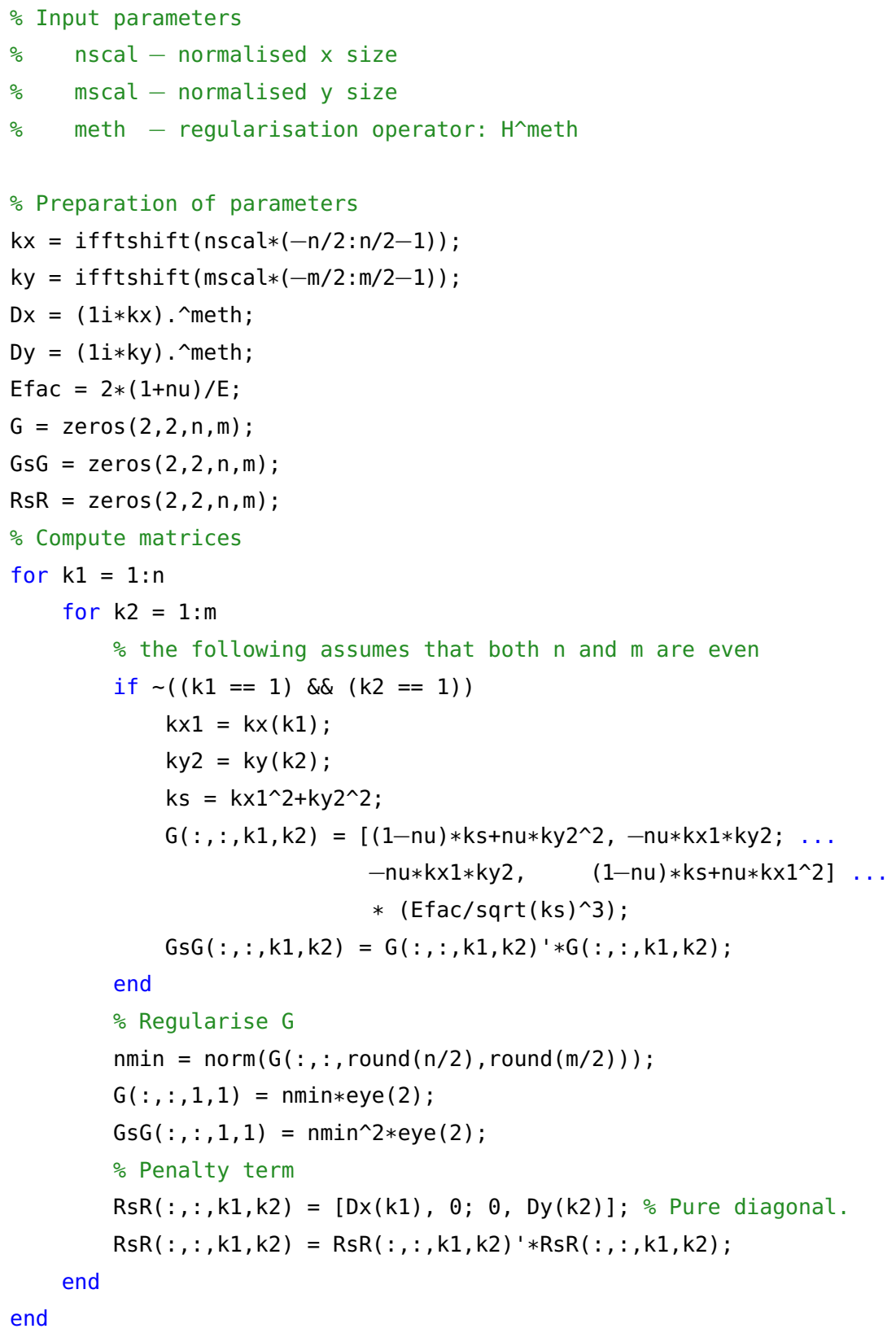

end

\footnotetext{
$\%$ Function to for FTTC

\% Copyright 02018 Jana Hanke, Version 23rd February
} 


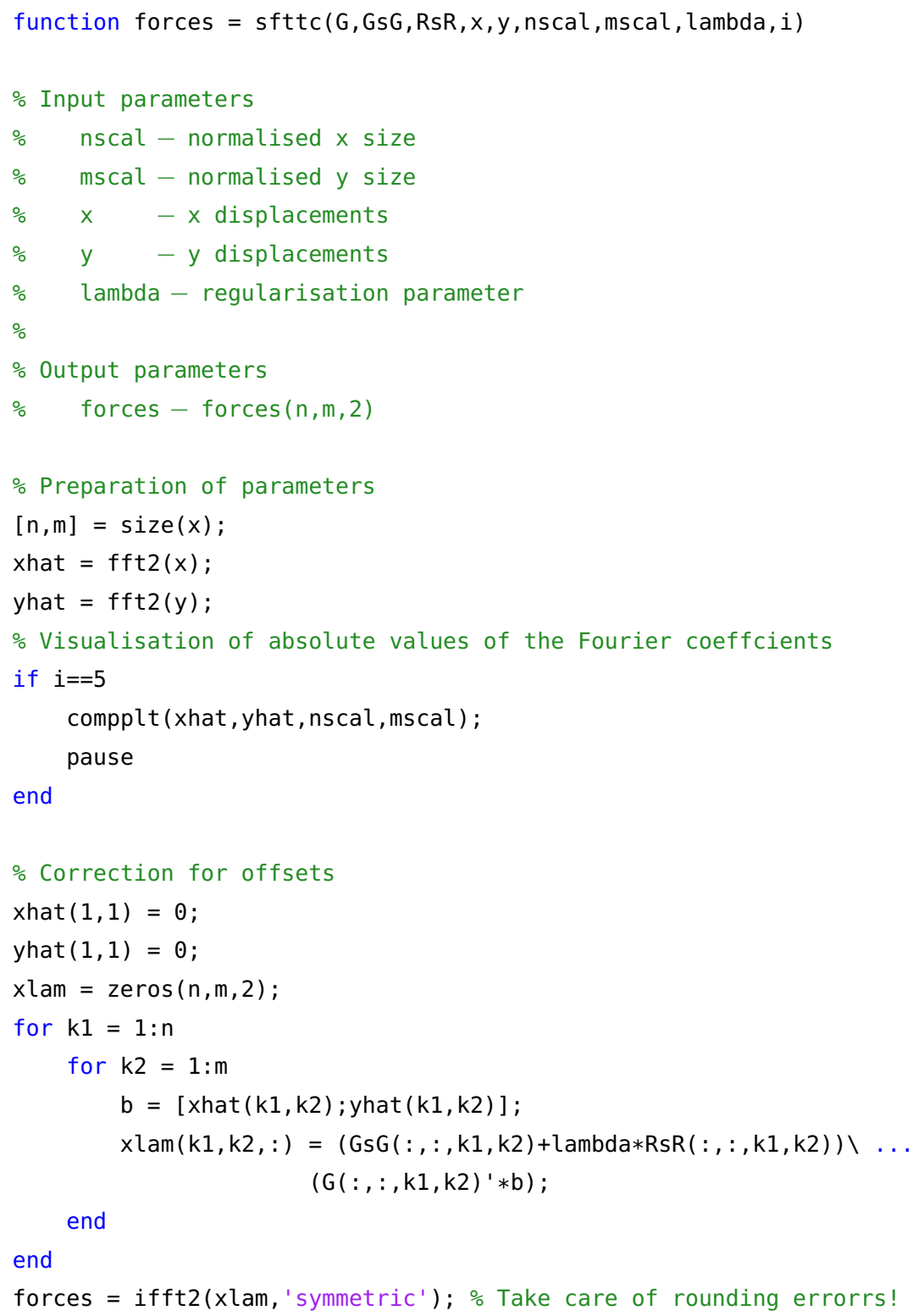




\section{Bibliography}

[1] P. Aarts, P. Bolhuis, K. S. Sakariassen, R. Heethaar, and J. Sixma. Red blood cell size is important for adherence of blood platelets to artery subendothelium. Blood, 62(1):214-217, 1983.

[2] D. Aquino, A. Schönle, C. Geisler, C. V. Middendorff, C. A. Wurm, Y. Okamura, T. Lang, S. W. Hell, and A. Egner. Two-color nanoscopy of threedimensional volumes by 4 pi detection of stochastically switched fluorophores. Nature Methods, 8(4):353-359, 2011.

[3] Y. Aratyn-Schaus, P. W. Oakes, and M. L. Gardel. Dynamic and structural signatures of lamellar actomyosin force generation. Molecular Biology of the Cell, 22(8):1330-1339, 2011.

[4] R. D. Averett, B. Menn, E. H. Lee, C. C. Helms, T. Barker, and M. Guthold. A modular fibrinogen model that captures the stress-strain behavior of fibrin fibers. Biophysical Journal, 103(7):1537-1544, oct 2012.

[5] S. Banerjee and M. C. Marchetti. Contractile stresses in cohesive cell layers on finite-thickness substrates. Physical Review Letters, 109(10):108101, 2012.

[6] D. J. Beebe, G. A. Mensing, and G. M. Walker. Physics and applications of microfluidics in biology. Annual Review of Biomedical Engineering, 4:261286, 2002.

[7] L. Boldock, C. Wittkowske, and C. M. Perrault. Microfluidic traction force microscopy to study mechanotransduction in angiogenesis. Microcirculation (New York, N.Y. : 1994), 24(5), 2017.

[8] J.-Y. Bouguet. Pyramidal implementation of the affine lucas kanade feature tracker: Description of the algorithm. Intel Corporation, 2001.

[9] R. Bulirsch and J. Stoer. Introduction to Numerical Analysis, volume Texts in Applied Mathematics. Springer New York, 2010.

[10] J. P. Butler, I. M. Tolic-Norrelykke, B. Fabry, and J. J. Fredberg. Traction fields, moments, and strain energy that cells exert on their surroundings. AJP: Cell Physiology, 282(3):C595-C605, 2002. 
[11] Y. Cai, N. Biais, G. Giannone, M. Tanase, G. Jiang, J. M. Hofman, C. H. Wiggins, P. Silberzan, A. Buguin, B. Ladoux, and M. P. Sheetz. Nonmuscle myosin IIA-dependent force inhibits cell spreading and drives f-actin flow. Biophysical Journal, 91(10):3907-3920, nov 2006.

[12] J. P. Califano and C. A. Reinhart-King. Substrate stiffness and cell area predict cellular traction stresses in single cells and cells in contact. Cellular and Molecular Bioengineering, 3(1):68-75, 2010.

[13] B. G. Chung, L. A. Flanagan, S. W. Rhee, P. H. Schwartz, A. P. Lee, E. S. Monuki, and N. L. Jeon. Human neural stem cell growth and differentiation in a gradient-generating microfluidic device. Lab on a Chip, 5(4):401, 2005.

[14] M. Collot, R. Kreder, A. L. Tatarets, L. D. Patsenker, Y. Mely, and A. S. Klymchenko. Bright fluorogenic squaraines with tuned cell entry for selective imaging of plasma membrane vs. endoplasmic reticulum. Chemical Communications, 51(96):17136-17139, 2015.

[15] Comsol Multiphysics. User's Guide CFD Module, 112013.

[16] C. G. Conant, M. A. Schwartz, J. E. Beecher, R. C. Rudoff, C. IonescuZanetti, and J. T. Nevill. Well plate microfluidic system for investigation of dynamic platelet behavior under variable shear loads. Biotechnology and Bioengineering, 108(12):2978-2987, jul 2011.

[17] T. Das, T. K. Maiti, and S. Chakraborty. Traction force microscopy on-chip: shear deformation of fibroblast cells. Lab on a Chip, 8(8):1308, 2008.

[18] A. B. V. de Walle, J. Fontenot, T. G. Spain, D. B. Brunski, E. S. Sanchez, J. C. Keay, M. E. Curtis, M. B. Johnson, T. A. Snyder, and D. W. Schmidtke. The role of fibrinogen spacing and patch size on platelet adhesion under flow. Acta Biomaterialia, 8(11):4080-4091, nov 2012.

[19] E. Décave, J. D. D. Rieu, S. Fache, Y. Bréchet, B. Fourcade, M. Satre, and F. Bruckert. Shear flow-induced motility of dictyostelium discoideum cells on solid substrate. Journal of Cell Science, 116(21):4331-4343, nov 2003.

[20] H. Delanoë-Ayari, S. Iwaya, Y. T. Maeda, J. Inose, C. Rivière, M. Sano, and J.-P. Rieu. Changes in the magnitude and distribution of forces at different dictyostelium developmental stages. Cell Motility and the Cytoskeleton, 65(4):314-331, 2008.

[21] H. Delanoë-Ayari, J. P. Rieu, and M. Sano. 4d traction force microscopy reveals asymmetric cortical forces in migrating dictyostelium cells. Physical Review Letters, 105(24):248103, 2010. 
[22] B. Diagouraga, A. Grichine, A. Fertin, J. Wang, S. Khochbin, and K. Sadoul. Motor-driven marginal band coiling promotes cell shape change during platelet activation. The Journal of Cell Biology, 204(2):177-185, 2014.

[23] M. Diez-Silva, M. Dao, J. Han, C.-T. Lim, and S. Suresh. Shape and biomechanical characteristics of human red blood cells in health and disease. MRS Bulletin, 35(05):382-388, may 2010.

[24] D. C. Duffy, J. C. McDonald, O. J. A. Schueller, and G. M. Whitesides. Rapid prototyping of microfluidic systems in poly(dimethylsiloxane). Analytical Chemistry, 70(23):4974-4984, dec 1998.

[25] T. Dullweber. Zytoskeletale Reorganisation in humanen Blutplättchen: Cytoskeletal Reorganisation in Human Blood Platelets. Bachelor's thesis, GeorgAugust University Göttingen, Göttingen, 2015.

[26] C. M. Edwards and U. S. Schwarz. Force localization in contracting cell layers. Physical Review Letters, 107(12):128101, 2011.

[27] H. W. Engl, M. Hanke, and A. Neubauer. Regularization of inverse problems, volume v.375 of Mathematics and its applications. Kluwer Academic, Dordrecht and London, 1996.

[28] A. J. Engler, S. Sen, H. L. Sweeney, and D. E. Discher. Matrix elasticity directs stem cell lineage specification. Cell, 126(4):677-689, 2006.

[29] S. Feghhi, W. W. Tooley, and N. J. Sniadecki. Nonmuscle myosin IIA regulates platelet contractile forces through rho kinase and myosin light-chain kinase. Journal of Biomechanical Engineering, 138(10):104506, sep 2016.

[30] R. W. Fox, P. J. Pritchard, and A. T. McDonald. Fox and McDonald's introduction to fluid mechanics. John Wiley \& Sons Inc. and John Wiley [distributor], Hoboken NJ and Chichester, 8th ed. edition, 2011.

[31] A. Freikamp, A.-L. Cost, and C. Grashoff. The piconewton force awakens: Quantifying mechanics in cells. Trends in Cell Biology, 26(11):838-847, nov 2016.

[32] S. García, R. Sunyer, A. Olivares, J. Noailly, J. Atencia, and X. Trepat. Generation of stable orthogonal gradients of chemical concentration and substrate stiffness in a microfluidic device. Lab on a Chip, 15(12):2606-2614, 2015.

[33] M. Guizar-Sicairos, S. T. Thurman, and J. R. Fienup. Efficient subpixel image registration algorithms. Optics Letters, 33(2):156, 2008. 
[34] E. Gutierrez, B. G. Petrich, S. J. Shattil, M. H. Ginsberg, A. Groisman, and A. Kasirer-Friede. Microfluidic devices for studies of shear-dependent platelet adhesion. Lab on a Chip, 8(9):1486, 2008.

[35] S. J. Han, K. S. Bielawski, L. H. Ting, M. L. Rodriguez, and N. J. Sniadecki. Decoupling substrate stiffness, spread area, and micropost density: A close spatial relationship between traction forces and focal adhesions. Biophysical Journal, 103(4):640-648, 2012.

[36] J. Hanke, D. Probst, A. Zemel, U. Schwarz, and S. Köster. Dynamics of force generation by spreading platelets. Soft Matter, 14(31):6571-6581, 2018.

[37] J. Hanke, C. Ranke, E. Perego, and S. Köster. Human blood platelets contract in perpendicular direction to shear flow. Under review.

[38] R. R. Hansen, A. A. Tipnis, T. C. White-Adams, J. A. D. Paola, and K. B. Neeves. Characterization of collagen thin films for von willebrand factor binding and platelet adhesion. Langmuir, 27(22):13648-13658, nov 2011.

[39] R. R. Hansen, A. R. Wufsus, S. T. Barton, A. A. Onasoga, R. M. JohnsonPaben, and K. B. Neeves. High content evaluation of shear dependent platelet function in a microfluidic flow assay. Annals of Biomedical Engineering, 41(2):250-262, sep 2012.

[40] C. R. Harmison, R. H. Landaburu, and W. H. Seegers. Some physicochemical properties of bovine thrombin. The Journal of Biological Chemistry, 236:1693-1696, 1961.

[41] A. Harris, P. Wild, and D. Stopak. Silicone rubber substrata: A new wrinkle in the study of cell locomotion. Science, 208(4440):177-179, 1980.

[42] M. T. Heath. Scientific computing: An introductory survey / Michael T. Heath. McGraw Hill, Boston, Mass. and London, 2nd ed. edition, 2002.

[43] N. Hersch, B. Wolters, G. Dreissen, R. Springer, N. Kirchgessner, R. Merkel, and B. Hoffmann. The constant beat: cardiomyocytes adapt their forces by equal contraction upon environmental stiffening. Biology Open, 2(3):351361, feb 2013.

[44] C. Hirsch. Numerical Computation of Internal and External Flows: The Fundamentals of Computational Fluid Dynamics. Butterworth-Heinemann, 2007.

[45] C. N. Holenstein, U. Silvan, and J. G. Snedeker. High-resolution traction force microscopy on small focal adhesions - improved accuracy through optimal marker distribution and optical flow tracking. Scientific Reports, $7: 41633,2017$. 
[46] K. L. Hui, L. Balagopalan, L. E. Samelson, and A. Upadhyaya. Cytoskeletal forces during signaling activation in jurkat t-cells. Molecular Biology of the Cell, 26(4):685-695, 2015.

[47] S. S. Hur, J. C. del Álamo, J. S. Park, Y.-S. Li, H. A. Nguyen, D. Teng, K.-C. Wang, L. Flores, B. Alonso-Latorre, J. C. Lasheras, and S. Chien. Roles of cell confluency and fluid shear in 3-dimensional intracellular forces in endothelial cells. Proceedings of the National Academy of Sciences of the United States of America, 109(28):11110-11115, 2012.

[48] A. Itakura, J. E. Aslan, S. Sinha, T. C. White-Adams, I. A. Patel, R. MezaRomero, A. A. Vandenbark, G. G. Burrows, H. Offner, and O. J. McCarty. Characterization of human platelet binding of recombinant $t$ cell receptor ligand. Journal of Neuroinflammation, 7:75, 2010.

[49] J. E. Italiano, P. Lecine, R. A. Shivdasani, and J. H. Hartwig. Blood platelets are assembled principally at the ends of proplatelet processes produced by differentiated megakaryocytes. The Journal of Cell Biology, 147(6):12991312, dec 1999.

[50] R. A. Jannat, M. Dembo, and D. A. Hammer. Traction forces of neutrophils migrating on compliant substrates. Biophysical Journal, 101(3):575-584, aug 2011.

[51] R. Kaunas, P. Nguyen, S. Usami, and S. Chien. From the cover: Cooperative effects of rho and mechanical stretch on stress fiber organization. Proceedings of the National Academy of Sciences of the United States of America, 102(44):15895-15900, oct 2005.

[52] S. Kim, H. J. Kim, and N. L. Jeon. Biological applications of microfluidic gradient devices. Integrative Biology, 2(11-12):584, 2010.

[53] A. Kirsch. An introduction to the mathematical theory of inverse problems, volume 120 of Applied mathematical sciences. Springer, New York, 2nd ed. edition, 2011.

[54] A. Knight. Single molecule biology. Elsevier Academic Press, London, 2009.

[55] C. M. Kraning-Rush, J. P. Califano, and C. A. Reinhart-King. Cellular traction stresses increase with increasing metastatic potential. PLOS ONE, 7(2):e32572, 2012.

[56] E. Kröner. Allgemeine kontinuumstheorie der versetzungen und eigenspannungen. Archive for Rational Mechanics and Analysis, 4(1):273-334, 1959. 
[57] R. H. W. Lam, Y. Sun, W. Chen, and J. Fu. Elastomeric microposts integrated into microfluidics for flow-mediated endothelial mechanotransduction analysis. Lab on a Chip, 12(10):1865-1873, 2012.

[58] W. A. Lam, O. Chaudhuri, A. Crow, K. D. Webster, T.-D. Li, A. Kita, J. Huang, and D. A. Fletcher. Mechanics and contraction dynamics of single platelets and implications for clot stiffening. Nature Materials, 10(1):61-66, 2010.

[59] L. D. Landau, L. P. Pitaevskii, A. M. Kosevich, and E. M. Lifshitz. Theory of Elasticity. Elsevier Science, 2012.

[60] H. Lee, G. Kim, C. Lim, B. Lee, and S. Shin. A simple method for activating the platelets used in microfluidic platelet aggregation tests: Stirring-induced platelet activation. Biomicrofluidics, 10(6):064118, nov 2016.

[61] W. R. Legant, C. K. Choi, J. S. Miller, L. Shao, L. Gao, E. Betzig, and C. S. Chen. Multidimensional traction force microscopy reveals out-of-plane rotational moments about focal adhesions. Proceedings of the National Academy of Sciences of the United States of America, 110(3):881-886, 2013.

[62] W. R. Legant, J. S. Miller, B. L. Blakely, D. M. Cohen, G. M. Genin, and C. S. Chen. Measurement of mechanical tractions exerted by cells in threedimensional matrices. Nature Methods, 7(12):969-971, 2010.

[63] J. Lembong, B. Sabass, B. Sun, M. E. Rogers, and H. A. Stone. Mechanics regulates ATP-stimulated collective calcium response in fibroblast cells. Journal of The Royal Society Interface, 12(108):20150140, jun 2015.

[64] Y. Li, Z. Hu, and C. Li. New method for measuring poisson's ratio in polymer gels. Journal of Applied Polymer Science, 50(6):1107-1111, 1993.

[65] X. M. Liang, S. J. Han, J.-A. Reems, D. Gao, and N. J. Sniadecki. Platelet retraction force measurements using flexible post force sensors. Lab on a Chip, 10(8):991, 2010.

[66] R. I. Litvinov, A. Mekler, H. Shuman, J. S. Bennett, V. Barsegov, and J. W. Weisel. Resolving two-dimensional kinetics of the integrin $\alpha \operatorname{Ib} \beta 3$-fibrinogen interactions using binding-unbinding correlation spectroscopy. Journal of Biological Chemistry, 287(42):35275-35285, aug 2012.

[67] R. I. Litvinov, H. Shuman, J. S. Bennett, and J. W. Weisel. Binding strength and activation state of single fibrinogen-integrin pairs on living cells. Proceedings of the National Academy of Sciences of the United States of America, 99(11):7426-7431, may 2002. 
[68] W. Liu, C. R. Carlisle, E. A. Sparks, and M. Guthold. The mechanical properties of single fibrin fibers. Journal of Thrombosis and Haemostasis, jan 2010.

[69] C.-M. Lo, H.-B. Wang, M. Dembo, and Y.-L. Wang. Cell movement is guided by the rigidity of the substrate. Biophysical Journal, 79:144-152, 2000.

[70] S. N. Lophaven, H. B. Nielsen, and J. Søndergaards. Aspects of the matlab toolbox dace. Technical report, Technical University of Denmark, 2002.

[71] B. D. Lucas and T. Kanade. An iterative image registration technique with an application to stereo vision. In Intl. Joint Conf. on Artificial Intelligence, Vancouver, British Columbia, 1981.

[72] M. A. Lukas. Strong robust generalized cross-validation for choosing the regularization parameter. Inverse Problems, 24(3):034006, 2008.

[73] G. Lukinavičius, L. Reymond, E. D'Este, A. Masharina, F. Göttfert, H. Ta, A. Güther, M. Fournier, S. Rizzo, H. Waldmann, C. Blaukopf, C. Sommer, D. W. Gerlich, H.-D. Arndt, S. W. Hell, and K. Johnsson. Fluorogenic probes for live-cell imaging of the cytoskeleton. Nature Methods, 11(7):731$733,2014$.

[74] K. Mandal, I. Wang, E. Vitiello, L. A. C. Orellana, and M. Balland. Cell dipole behaviour revealed by ECM sub-cellular geometry. Nature Communications, 5:5749, dec 2014.

[75] W. A. Marganski, M. Dembo, and Y.-L. Wang. Measurements of cellgenerated deformations on flexible substrata using correlation-based optical flow. Methods in Enzymology, 361, 2003.

[76] J. C. McDonald and G. M. Whitesides. Poly(dimethylsiloxane) as a material for fabricating microfluidic devices. Accounts of Chemical Research, 35(7):491-499, jul 2002.

[77] A. D. Michelson. Platelets. Elsevier/Academic Press, Amsterdam and London, 2nd ed. edition, 2007.

[78] D. Mitrossilis, J. Fouchard, A. Guiroy, N. Desprat, N. Rodriguez, B. Fabry, and A. Asnacios. Single-cell response to stiffness exhibits muscle-like behavior. Proceedings of the National Academy of Sciences of the United States of America, 106(43):18243-18248, oct 2009.

[79] R. E. Mott and B. P. Helmke. Mapping the dynamics of shear stress-induced structural changes in endothelial cells. American Journal of Physiology-Cell Physiology, 293(5):C1616-C1626, nov 2007. 
[80] S. Munevar, Y.-L. Wang, and M. Dembo. Traction force microscopy of migrating normal and h-ras transformed 3t3 fibroblasts. Biophysical Journal, 80(4):1744-1757, 2001.

[81] D. R. Myers, Y. Qiu, M. E. Fay, M. Tennenbaum, D. Chester, J. Cuadrado, Y. Sakurai, J. Baek, R. Tran, J. C. Ciciliano, B. Ahn, R. G. Mannino, S. T. Bunting, C. Bennett, M. Briones, A. Fernandez-Nieves, M. L. Smith, A. C. Brown, T. Sulchek, and W. A. Lam. Single-platelet nanomechanics measured by high-throughput cytometry. Nature Materials, 16(2):230-235, oct 2016.

[82] K. B. Neeves and S. L. Diamond. A membrane-based microfluidic device for controlling the flux of platelet agonists into flowing blood. Lab on a Chip, 8(5):701, 2008.

[83] K. B. Neeves, S. F. Maloney, K. P. Fong, A. A. Schmaier, M. L. Kahn, L. F. Brass, and S. L. Diamond. Microfluidic focal thrombosis model for measuring murine platelet deposition and stability: PAR4 signaling enhances shearresistance of platelet aggregates. Journal of Thrombosis and Haemostasis, 6(12):2193-2201, dec 2008.

[84] W. S. Nesbitt, S. Kulkarni, S. Giuliano, I. Goncalves, S. M. Dopheide, C. L. Yap, I. S. Harper, H. H. Salem, and S. P. Jackson. Distinct glycoprotein ib/v/IX and integrin $\alpha \mathrm{IIb} \beta 3$-dependent calcium signals cooperatively regulate platelet adhesion under flow. Journal of Biological Chemistry, 277(4):2965-2972, nov 2001.

[85] C. P. Ng and M. A. Swartz. Fibroblast alignment under interstitial fluid flow using a novel 3-d tissue culture model. American Journal of Physiology-Heart and Circulatory Physiology, 284(5):H1771-H1777, may 2003.

[86] C. P. Ng and M. A. Swartz. Mechanisms of interstitial flow-induced remodeling of fibroblast-collagen cultures. Annals of Biomedical Engineering, 34(3):446-454, feb 2006.

[87] N. Nisenholz, K. Rajendran, Q. Dang, H. Chen, R. Kemkemer, R. Krishnan, and A. Zemel. Active mechanics and dynamics of cell spreading on elastic substrates. Soft Matter, 10(37):7234, 2014.

[88] P. W. Oakes, S. Banerjee, M. C. Marchetti, and M. L. Gardel. Geometry regulates traction stresses in adherent cells. Biophysical Journal, 107(4):825833, 2014.

[89] P. W. Oakes, D. C. Patel, N. A. Morin, D. P. Zitterbart, B. Fabry, J. S. Reichner, and J. X. Tang. Neutrophil morphology and migration are affected by substrate elasticity. Blood, 114(7):1387-1395, jun 2009. 
[90] A. Paknikar. Cytoskeletal Reorganization in Human Blood Platelets During Spreading. Dissertation, Georg-August University Göttingen, Göttingen, 2016.

[91] T. G. Papaioannou and C. Stefanadis. Vascular wall shear stress: basic principles and methods. Hellenic Journal of Cardiology : HJC $=$ Hellenike kardiologike epitheorese, 46(1):9-15, 2005.

[92] C. M. Perrault, A. Brugues, E. Bazellieres, P. Ricco, D. Lacroix, and X. Trepat. Traction forces of endothelial cells under slow shear flow. Biophysical Journal, 109(8):1533-1536, 2015.

[93] Y. Qiu, A. C. Brown, D. R. Myers, Y. Sakurai, R. G. Mannino, R. Tran, B. Ahn, E. T. Hardy, M. F. Kee, S. Kumar, G. Bao, T. H. Barker, and W. A. Lam. Platelet mechanosensing of substrate stiffness during clot formation mediates adhesion, spreading, and activation. Proceedings of the National Academy of Sciences of the United States of America, 111(40):14430-14435, 2014.

[94] Y. Qiu, J. Ciciliano, D. R. Myers, R. Tran, and W. A. Lam. Platelets and physics: How platelets "feel" and respond to their mechanical microenvironment. Blood Reviews, 29(6):377-386, nov 2015.

[95] M. Raffel, C. E. Willert, and J. Kompenhans. Particle image velocimetry: A practical guide / Markus Raffel, Christian E. Willert, Jürgen Kompenhans. Experimental fluid mechanics. Springer, Berlin and London, 1998.

[96] C. Ranke. Development of a Tool to Study Human Blood Platelets under Physiological Flow Conditions. Master's dissertation, Georg-August University Göttingen, Göttingen, 2016.

[97] A. I. Ruban and J. S. B. Gajjar. Fluid Dynamics. Oxford University Press, 2014.

[98] B. Sabass, M. L. Gardel, C. M. Waterman, and U. S. Schwarz. High resolution traction force microscopy based on experimental and computational advances. Biophysical Journal, 94(1):207-220, 2008.

[99] K. S. Sakariassen, L. Orning, and V. T. Turitto. The impact of blood shear rate on arterial thrombus formation. Future Science OA, 1(4):FSO30, 2015.

[100] R. Sandmann. Blood Platelet Behavior on Structured Substrates - From Spreading Dynaics to Cell Morphology. PhD thesis, Georg-AugustUniversität Göttingen, 2015.

[101] R. Sandmann and S. Köster. Topographic cues reveal two distinct spreading mechanisms in blood platelets. Scientific Reports, 6:22357, 2016. 
[102] R. Sandmann, S. Schwarz Henriques, F. Rehfeldt, and S. Köster. Microtopography influences blood platelet spreading. Soft Matter, 10(14):2365$2371,2014$.

[103] B. Savage, E. Saldívar, and Z. M. Ruggeri. Initiation of platelet adhesion by arrest onto fibrinogen or translocation on von willebrand factor. Cell, 84(2):289-297, jan 1996.

[104] U. S. Schwarz and S. A. Safran. Elastic interactions of cells. Physical Review Letters, 88(4):048102, 2002.

[105] U. S. Schwarz and J. R. Soiné. Traction force microscopy on soft elastic substrates: A guide to recent computational advances. Biochimica et Biophysica Acta (BBA) - Molecular Cell Research, 1853(11):3095-3104, 2015.

[106] S. Schwarz Henriques. Biophysics of Blood Platelet Contraction. Dissertation, Georg-August-Universität Göttingen, Göttingen, 2012.

[107] S. Schwarz Henriques, R. Sandmann, A. Strate, and S. Köster. Force field evolution during human blood platelet activation. Journal of Cell Science, 125(16):3914-3920, 2012.

[108] J. Seifert, J. Rheinlaender, F. Lang, M. Gawaz, and T. E. Schäffer. Thrombin-induced cytoskeleton dynamics in spread human platelets observed with fast scanning ion conductance microscopy. Scientific Reports, $7(1): 4810,2017$.

[109] J. Shi and C. Tomasi. Good features to track. In IEEE Conference on Computer Vision and Pattern Recognition, pages 593-600, June 1994.

[110] J. R. D. Soiné, C. A. Brand, J. Stricker, P. W. Oakes, M. L. Gardel, and U. S. Schwarz. Model-based traction force microscopy reveals differential tension in cellular actin bundles. PLoS Computational Biology, 11(3):e1004076, 2015.

[111] S. Sorrentino, J.-D. Studt, O. Medalia, and K. T. Sapra. Roll, adhere, spread and contract: Structural mechanics of platelet function. European Journal of Cell Biology, 94(3-4):129-138, mar 2015.

[112] R. Steward, D. Tambe, C. C. Hardin, R. Krishnan, and J. J. Fredberg. Fluid shear, intercellular stress, and endothelial cell alignment. American Journal of Physiology-Cell Physiology, 308(8):C657-C664, apr 2015.

[113] T. Stocker. Introduction to Climate Modelling. Springer-Verlag GmbH, 2011.

[114] J. Stricker, B. Sabass, U. S. Schwarz, and M. L. Gardel. Optimization of traction force microscopy for micron-sized focal adhesions. Journal of Physics: Condensed Matter, 22(19):194104, apr 2010. 
[115] R. W. Style, R. Boltyanskiy, G. K. German, C. Hyland, C. W. MacMinn, A. F. Mertz, L. A. Wilen, Y. Xu, and E. R. Dufresne. Traction force microscopy in physics and biology. Soft Matter, 10(23):4047-4055, 2014.

[116] J. L. Tan, J. Tien, D. M. Pirone, D. S. Gray, K. Bhadriraju, and C. S. Chen. Cells lying on a bed of microneedles: An approach to isolate mechanical force. Proceedings of the National Academy of Sciences of the United States of America, 100(4):1484-1489, 2003.

[117] H. Tanimoto and M. Sano. A simple force-motion relation for migrating cells revealed by multipole analysis of traction stress. Biophysical Journal, 106(1):16-25, jan 2014.

[118] A. Tokarev, A. Butylin, and F. Ataullakhanov. Platelet adhesion from shear blood flow is controlled by near-wall rebounding collisions with erythrocytes. Biophysical Journal, 100(4):799-808, feb 2011.

[119] C. Tomasi and T. Kanade. Detection and tracking of point features.

[120] L. Trichet, J. Le Digabel, R. J. Hawkins, S. R. K. Vedula, M. Gupta, C. Ribrault, P. Hersen, R. Voituriez, and B. Ladoux. Evidence of a large-scale mechanosensing mechanism for cellular adaptation to substrate stiffness. Proceedings of the National Academy of Sciences of the United States of America, 109(18):6933-6938, 2012.

[121] W. R. Trickey, F. P. Baaijens, T. A. Laursen, L. G. Alexopoulos, and F. Guilak. Determination of the poisson's ratio of the cell: recovery properties of chondrocytes after release from complete micropipette aspiration. Journal of Biomechanics, 39(1):78-87, jan 2006.

[122] Q. Tseng, I. Wang, E. Duchemin-Pelletier, A. Azioune, N. Carpi, J. Gao, O. Filhol, M. Piel, M. Théry, and M. Balland. A new micropatterning method of soft substrates reveals that different tumorigenic signals can promote or reduce cell contraction levels. Lab on a Chip, 11(13):2231, 2011.

[123] J. W. Tukey. An introduction to the calculations of numerical spectrum analysis. Advanced Seminar in Spectral Analysis of Time Series. Wiley, New York, 1967.

[124] G. Velve-Casquillas, M. L. Berre, M. Piel, and P. T. Tran. Microfluidic tools for cell biological research. Nano Today, 5(1):28-47, feb 2010.

[125] Y. Wang, D. N. LeVine, M. Gannon, Y. Zhao, A. Sarkar, B. Hoch, and $\mathrm{X}$. Wang. Force-activatable biosensor enables single platelet force mapping directly by fluorescence imaging. Biosensors and Bioelectronics, 100:192200, feb 2018. 
[126] A. Zemel, F. Rehfeldt, A. E. X. Brown, D. E. Discher, and S. A. Safran. Cell shape, spreading symmetry and the polarization of stress-fibers in cells. Journal of Physics. Condensed Matter : An Institute of Physics Journal, 22(19):194110, 2010.

[127] Y. Zhang, C. Ge, C. Zhu, and K. Salaita. DNA-based digital tension probes reveal integrin forces during early cell adhesion. Nature Communications, $5: 5167$, oct 2014 .

[128] Y. Zhang, Y. Qiu, A. T. Blanchard, Y. Chang, J. M. Brockman, V. P.Y. Ma, W. A. Lam, and K. Salaita. Platelet integrins exhibit anisotropic mechanosensing and harness piconewton forces to mediate platelet aggregation. Proceedings of the National Academy of Sciences of the United States of America, 115(2):325-330, dec 2017. 


\section{Acknowledgements}

At the end of this thesis, I would like to thank the people who have contributed in one way or another to make this work possible.

First, I would like to thank my supervisor Prof. Sarah Köster for giving me the opportunity to conduct my thesis in her group. Our regular meetings to discuss the results and further proceedings have helped greatly to bring this project forward. Thank you for letting me try out my ideas until it worked as I wished it to be and helping with ideas when it did not.

For their agreement to become a member of my thesis committee, I thank Prof. Claudia Steinem and Prof. Stefan Hell. Even when the organisation of our meetings sometimes proved difficult due to their busy schedules, they always found time for it. These meetings brought fruitful discussions of my work and provided me the opportunity to consider my problems through completely different eyes. An additional thank you goes to Prof. Steinem for taking the time to review my thesis. I would also like to thank Prof. Anette Zippelius, Prof. Jörg Enderlein and Dr. Florian Rehfeld for agreeing to work in the examination committee.

For their valuable contributions in theoretical parts of this thesis, I thank Dimitri Probst, Prof. Ulrich Schwarz (both University of Heidelberg) and Dr. Assaf Zemel (Hebrew University of Jerusalem). I learnt plenty during our discussions about data analysis and later the modelling of the platelets. Thank you for taking the time for all the long discussions on how to proceed best, be it via Skype, long email conversations or visits in Heidelberg.

The whole project would not have been possible without the team of the blood donation centre at the University Hospital Göttingen who provided us with their expired blood preservatives. I appreciate the help of the entire team, especially the patience of the nurses when I called each morning anew in hopes of new samples. While the work I conducted at the Third Institute of Physics is not presented in this work, I would still like to thank Dr. Anna Chizhik, Daja Ruhland and Dr. Alexej Chizhik for their support in the MIET experiments. Our results are still not conclusive but I am sure that this will change soon. Thank you to Anna for helping me take the images shown in Fig. 2.3. 
The work in this thesis would not have been possible without the support of the entire group at the Institute of X-Ray Physics. First, a big thank you to Dr. Rabea Sandmann, Dr. Aishwarya Paknikar, Christiane Ranke, Anna Zelena, Tim Dullweber and Robert Hammermann for all our discussions on the nature of platelets and how to work with them. I learnt much from you, both through your own experience that you gladly shared and your own questions and ideas. I am grateful to Christiane and Gerrit Brehm for providing me with the first set of wavers for the microfluidic devices. The smooth work of the microfluidic experiments would not have been possible without Eleonora Perego and Irina Iriady. Eleonora, thanks for all your work and patience and taking the time to provide me with new wavers when I managed to break some of the old ones. Irina, I appreciate all your late nights in the lab when you took over parts of the substrate production for the microfluidic experiments. I could not have finish this part of the thesis that fast without you. I am indebted to Manuela Denz, Johanna Block and Julia Kraxner for all our discussions over a cup of tea and coffee. Your different research backgrounds helped me gain new ideas and insights. For their kind agreement to proof-read parts of this thesis, I would like to thank Manuela, Julia and Charlotta Lorenz. To all the technical staff, both in the lab and in the office, I appreciate your help and support. To all the aforementioned group members as well as all others, present and past, I appreciate all your support at work and friendship both in the lab and outside. I greatly enjoyed our small excursions, from hiking tours to trips to the theatre.

Last but not least, I would not have come so far without my family and friends whom supported me. Thanks for always lending an ear when needed and being no longer than a phone call away. To Rebekka, Julia and Ruth, I am grateful for all you enthusiasm and dragging me back to the ballet barre. Your creativity as teachers and friendship outside the training room made me forget all the stressful days at work and put a smile on my face at the end of the day. To Susi, thanks for all the fun conversations over a cup of coffee and the discussions about all things during our small excursions. Thank you for always listening and managing to make me laugh. To my parents, Tack för all eran stöd, uppmuntran och tålamod under åren, jag skulle inte har kommit så långt utan er. Tack för att ni alltid stödd mina beslut, även om det ledde till nästan tio års studier och ett antal flytt tvärs genom Europa. Jag hoppas ni är stolta över resultatet. 
$\mathrm{H}$ uman blood platelets are small, anucleate cell fragments that are important for the life-saving mechanism of haemostasis, i.e. the process to prevent a haemorrhage. Upon injury, blood platelets adhere to the exposed extra-cellular matrix and spread, clot and contract to close the wound. This process has to work reliably in a multitude of different environments, including surrounding tissues of various stiffnesses as well as the exposure of different shear flows due to blood circulation. In the present work, the reaction of human blood platelets to such environments is reported. Specifically, the spatial and temporal contraction behaviour of platelets and its variation is studied in response to elastic substrates within the stiffness range of $19 \mathrm{kPa}$ to $83 \mathrm{kPa}$ and shear rates between $14 \mathrm{~s} \wedge(-1)$ and $33 \mathrm{~s} \wedge(-1)$, corresponding to shear rates as found in larger veins.

To be able to visualise and analyse the contraction of the platelets, time-resolved traction force microscopy was employed together with a specifically tailored differential particle image velocimetry method and a microfluidic system. It was found that platelets exhibit very dynamic contractile behaviour as well as comparatively high forces in the light of their small size. The experimental results showed that platelets are not mechanosensitive within the studied stiffness or shear rate ranges. This was observed both in the spatial and temporal force development as well as the final spread area reached by the platelets. The force was, instead, depending on the spread area. By modelling the cell as an elastic, actively contracting disc and fitting this model to the dependency of the force on the area for data collected on various stiffnesses, it was concluded that due to their small size, platelets are only mechanosenstive to substrates of at least one order of magnitude softer than used here.

Under flow conditions, it was shown that platelets do indeed adapt to the direction of the flow. Here, the preferred orientation of the contraction in comparison to the flow direction changed from $45^{\circ}$ for the lower flow rates to roughly $90^{\circ}$ for the highest shear rate. To understand this result, the stress distribution on the platelets' surface under flow was simulated, demonstrating the highest stress to be found roughly at $90^{\circ}$. Hence, the reported orientation of the contraction is accounted for by the reduction of stress on the force-transmitting network of the platelet connected to the underlying substrate. In conclusion, platelets are mechanoinsensitive to a multitude of different environments, indicating an 'allor-nothing' response to such cues. 